NASA Technical Memorandum 83750

\title{
Analysis of Glow Discharges For Understanding The Process of Film Formation
}

M. Venugopalan

Western Illinois University

Macomb, Illinois

and

R. Avni

Lewis Research Center

Cleveland, Ohio

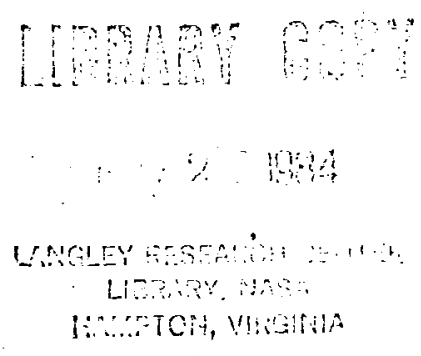

October 1984

\section{N/S}


ANALYSIS OF GLOW DISCHARGES FOR UNDERSTANDING

THE PROCESS OF FILM FORMATION*

\author{
M. Venugopalan \\ 31176013596722 \\ Western Illinois University \\ Department of Chemistry \\ Macomb, Illinols 61455 U.S.A. \\ and \\ R. Avnit \\ National Aeronautics and Space Administration \\ Lewis Research Center \\ Cleveland, Ohio 44135 U.S.A.
}

\title{
SUMMARY
}

The physical and chemical processes which occur during the formation of different types of films in a varlety of glow discharge plasmas are discussed. Emphasis is placed on plasma diagnostic experiments using spectroscopic methods, probe analysis, mass spectrometric sampling and magnetic resonance techniques which are.well suited to investigate the neutral and ionized gas phase specles as well as some aspects of plasma surface interactions. The results on metalilic, semi-conducting and insulating flims are reviewed in conjunction with proposed models and the problems encountered under film deposition conditions. It is concluded that the understanding of film deposition processes requires additional experimental information on plasma surface interactions of free radicals and the synergetic effects where photon, electron and ion bombardment change the reactivity of the incident radical with the surface.

\section{INTRODUCTION}

The transitory existence of atoms and free radicals has long been estab11shed in spectroscopy. Line spectra, of course, have their origin in free atoms, and investigations of band spectra have quite definitely shown the existence of free radicals. Generally, high temperature is needed to produce these particles, so the chemistry investigated is necessarily that of energetic species in the gas phase. Their high reactivity results in their rapid disappearance by reaction with themselves or with other substances which may be present. Often, condensation or deposition from vapor phase results on a surface, sometimes also known as a substrate, which relleves the species of their energies. If the coating thus formed is less than about $1 \times 10^{-6} \mathrm{~m}$ it is called a thin film. Clearly, the deposition process involves a phase transformation which can be understood from thermodynamics and kinetics istudies.

Because of important industrial applications thin film technology has grown rapidly during the past two decades or so. Originally, thin films were

*To be published as Chapter 4 in Thin Films From Free Atoms and Particles edited by J. Klabunde, Academic Press.

tBen Gurion University of the Negev, Nuclear Research Center Negev,

Beer Sheva, Israel and NRC-NASA Research Assoctate. 
produced by pyrolytic chemical vapor deposition (CVD) and deposition of inorganic or metal films from solution by plating, anodization, etc. Subsequently, physical methods were developed for deposition of films. One such method 1 makes use of "glow discharges" which produce a unique state of matter called plasma. In this chapter we will attempt a short review of the process of film formation as understood from analyses of glow discharge plasmas rather than of the films they produce. The latter topic, which has substantially contributed to the practical aspects of thin film technology will be covered in other chapters in this book. The reader is cautioned that descriptions of the plasma will be made in the context of the glow discharge process only. For a rigorous discussion of plasmas and plasma chemistry the reader is referred to a book edited by Venugopalan (1971).

\section{The Plasma State}

A plasma is a collection of charged and neutral particles resulting from the partial ionization of the atoms or molecules of a gas generally by an external electric fleld. In the types of plasmas discussed in this chapter, the degree of ionization is typically only $10^{-4}$, so the gas consists mostiy of neutrals. The Coulomb interaction of the charged particles with each other and with whatever electric (and magnetic) fields are externally applied is both strong and long-range so that collective behavior constitutes the prime characteristic of a plasma. The neutral particles, on the contrary, interact with each other and with the charged particles via the short range forces that come into play only during close encounters. The essential mechanisms in the plasma are excitation and relaxation and lonization and recombination. We may characterize the effects of all these interactions in terms of macroscopic variables such as pressure, density and temperature.

The plasma itself is virtualiy electric field free, i.e., equipotential. It is this potential that is called the plasma potential, $v_{p}$. The electron density, $n_{e}$, and ion density, $n_{1}$, are equal (on average) so that the plasma is quasi-neutral; their number. which is much less than the density of neutrals, is often known as the plasma density. Typical glow discharge plasmas have densities of $10^{9}-10^{12} \mathrm{~cm}^{-3}$. There is a distribution of electron energies, $f(E)$, which is at best described as non-Maxwellian. The average electron energles are around 2 to $10 \mathrm{eV}$ which corresponds to electron temperatures $T_{e}$ of $10^{4}-10^{5} \mathrm{~K}$. But the lons have only temperatures, $T_{1}$, somewhat above ambient, say $500 \mathrm{~K}$, so that there is a nonequilibrium nature of the plasma which leads to deposition of a film at much lower temperatures than are possible with pyrolytic CVD. This is the main reason for the sustained interest in the glow discharge as a means for film deposition.

Another reason we may cite is the possibility of using temperaturesensitive substrates. However, substrates immersed in a plasma acquire a negative floating potential with respect to the plasma. Consequently, the condensing species on the substrates would be subjected to ion bombardment, which can affect the heterogeneous reactions occurring on the substrate surface and thus influence the properties of the growing film. The ion

${ }^{1}$ In ion-beam deposition substrates are not immersed in a glow discharge, but are in a relatively good vaccum. 
Impact may set up a series of collisions between atoms of the substrate, possibly leading to the ejection or sputtering of one of these atoms. While sputtering is in itself a process used for the deposition of thin films, in glow discharges it may result in undesirable structural rearrangements of the deposited film. Nevertheless, by the application of glow discharge plasmas films of novel materials and commercial value have been produced. Further, plasma deposited fllms are conformal in nature and produce a lower pinhole density compared to "line-of-sight" deposition techniques such as evaporation and sputtering.

\section{Phenomena and Parameters Relevant to Film Formation}

In the previous chapter the theory of thin film formation was discussed. In general, the substrate w111 have a chemical nature different from that of the film material so that we must consider a third phase in which gaseous species are adsorbed on the substrate but have not yet reacted with other adsorbed species. Figure 1 shows a substrate indicating the adsorbed phase and the likely particles bombarding it when immersed in a glow discharge. Not only the nature and temperature of the substrate but also the adsorption sites on its surface are important in determining the nature of the deposited film. During the film growth, the substrate and growing film will be subject to many types of bombardment. A description of this will be attempted after the scenarlo of the glow discharge is presented in the next section.

Species impinging on the substrate may be adsorbed on the surface, then migrate, evaporate, or collide and combine eventually to form clusters in a process known as nucleation ( $f i g .2)$. This is the onset of a condensation process in which nuclet may contain tens or hundreds of atoms and typically have densities of $10^{10} \mathrm{~cm}^{-2}$. The build-up and/or growth of several nuclei may result in contact between nearest neighbors and so-called formation of islands of film material. Each 1 sland will contain one or a few crystallites. On a polycrystalline substrate, the orientation of each is land will be random, so that coalescence of the islands may produce a polycrystalitine film. ${ }^{2}$ Channels or holes, if any, during the coalescence stage fill via secondary nucleation to give a continuous $\mathrm{f} 1 \mathrm{~lm}$ ( $\mathrm{f} 1 \mathrm{~g} .2$ ). As in crystal growth, the mobile surface species tend to seek out low energy positions. Mobility is enhanced by increased surface temperature, but locating favorable lattice sites is time-consuming so that a high substrate temperature and low deposition rate lead to large grains, low density of crystal defects, and large film thickness for continuity.

Phenomena such as these are described in terms of the sticking coefficlent which is the ratio of the amount of species condensed on the substrate to the amount which has impinged. So far we have not delineated the plasma specles which may undergo these phenomena. of course, ions and electrons are present in any plasma. Recombination of positive ions and electrons gives rise to highly excited states that may dissociate into neutral fragments but more probably dissociate into radicals. A considerable body of analytical

2 In the case of a single crystal substrate, the island orientations may be determined by the substrate structure so that growth and coalescence leads to a single crystalline film. This is known as the phenomenon of epitaxy, described by Bauer and Poppa (1972). 
evidence has shown that a wide variety of free radicals are formed in glow discharges. Experiments and/or theory from the field of surface science offer nothing more than intuitive guidelines about the interaction of radicals with surfaces. It is, however, known that radicals frequently chemisorb on surfaces which appear inert to the parent molecule. In a recent discussion of this topic Winters (1980) has suggested that radicals have reasonably large sticking coefficients, but that they are not unity.

Figure 3 117ustrates the parameter problem relevant to film formation. It is obvious that the results of $f 11 \mathrm{~m}$ deposition are subject to large number of variables that are interdependent. For this reason experimental results from one deposition system to another are often not easily reproducible. The key insight that is needed is the identity, abundance and energy of all species incident on the substrate surface. This depends on the electrical element (plasma) of the system which in turn depends on a number of gas kinetic parameters. The complexity of polyatomic molecular gas discharges often used for thin films would appear to preclude quantitative information about species concentration being derived from $n_{e}$ and $f(E)$ even if these can be determined, since much of the cross section data for ionization/dissociation or ion-molecule and radical-molecule reactions does not presently exist. Thus, mechanistic understanding in relation to the plasma becomes difficult. But process control should be feasible by the use of fllm thickness/composition monitors, mass spectroscopy, and optical spectroscopy for detecting gas phase intermediates and for monitoring their concentration.

\section{GLOW DISCHARGES}

\section{Archltecture of Glow Discharges}

Consider a glass tube containing a gas at low pressure, say 1 torr. Into each end of the tube is inserted an electrode. Between the electrodes is connected a direct current (dc) power supply. When a voltage is first applied a very small current flows in random bursts (fig. 4). As the voltage is increased, the current increases steadily. As the current increases, the gas begins to glow visibly and the potential across the tube drops to a constant value. We say that a glow discharge has been established in the tube. The early work in gas discharges centered around the study of the visual behavior of the glow discharge, and led to a classification of the various regions illustrated in figure 5 . There are excellent descriptions of these in many textbooks such as Cobine (1958), von Engel (1965), and Nasser (1971). Before we analyse the spatial characteristics let us examine the voltage-current characteristics in some detail.

Ordinarily, there exists in a gas a small number of fons and electrons due to ionization by cosmic radiation. The initlal application of a dc potential causes these charges to move and cause random bursts of nearly constant current. The cathode emits electrons by a mechanism called field emission in ' which the electric field near the cathode surface extracts electrons directly from the metal. As the voltage is increased the charged particles acquire more energy, and undergo collistons with the electrodes and with neutral gas atoms producing more lons. If the voltage between the electrodes is high enough the ions striking the cathode can release electrons. This "secondary electron emission" causes the current to increase at a constant voltage 
known as the threshold or breakdown potentiali, $v_{m} \cdot{ }^{3}$ When the number of electrons produced is just sufficient to produce enough ions to regenerate the same number of electrons, a self-sustaining discharge is obtained. It is at this point the gas begins to "glow," the voltage drops, and the current rises abruptly (fig. 4).

The breakdown voltage, $v_{m}$, is crucial to the formation of the glow discharge. Its magnitude is governed, among others, by the interelectrode separation and the gas pressure which determines the mean free path of secondary electrons. If the pressure and/or separation are too large, ions generated in the gas are slowed by inelastic collisions and they strike the cathode with insufficient energy to produce secondary electrons. On the other hand, if the gas pressure is too low or the electrode separation too small, the secondaries cannot undergo a sufficlent number of lonizing colilisions before they are collected at the anode.

The secondary electron emission ratio of most materials is of the order of 0.1 so that several ions must bombard a given area of the cathode to produce another secondary electron. Initially, the bombardment is concentrated near th edges of the cathode or at other irregularities on the surface. As more power is supplied, the bombardment covers the whole cathode surface and a constant current is achieved. Further increases in power produce both increased voltage and current in a region known as the "abnormal glow" (fig. 4). This mode is usually used in virtually all glow discharge processes including film deposition. If thermionic emission occurs as a result of the cathode heating to a high temperature the discharge will transcend into an electrical arc characterized by a low-voltage high-current profile (fig. 4).

Within a glow discharge there exists distributions of potential, field, space charge, and current density as shown in figure 5. Visually these are seen as regions of varied luminosity. The application of a voltage primarily affects the region near the cathode, where it facilitates electron emission. Adjacent to the cathode, there is a narrow luminous region known as the cathode glow. The light emitted is characteristic of both the cathode material and the incident ion. Thus it is the region in which the positive ions formed at the cathode and the incoming discharge ions are neutralized by a variety of processes.

Secondary electrons are repelled at high velocity from the cathode and make collistons with neutrals at a distance away from the cathode corresponding to their mean free path. Because the electrons lose their energy by collisions nearly all of the applied voltage appears across this dark space. Since the mobility of lons is very much less than that of electrons, the predominant species in the dark space are ions. The high net positive space charge present in the cathode dark space forms a sheath and causes the sudden increase in potential between the cathode and the leading edge of the negative glow. This part of the potential is referred to as the cathode fall and is typicaliy 100 to $400 \mathrm{~V}$ in magnitude.

3 The term Townsend discharge, after the physicist who studied this phenomenon, is used to delineate this stage in the formation of a glow discharge. 
Acceleration of secondary electrons from the cathode results in fonizing collisions in the negative glow region. It so happens that most plasma deposition systems are essentially negative glow type discharges. The general consensus regarding the electron energy distribution in the negative glow is well summarized by Chapman (1980) as consisting of:

(1) Primary electrons with typical densities of $10^{6}$ or so at 1 torr pressure, which enter from the cathode sheath with nearly the full dark space potential and decay in energy primarily by inelastic collisions.

(2) Secondary electrons ( $n_{e}=10^{7}$ or so) of considerably lower energy, which are the product of ionizing collisions or primaries which have lost much of their energy.

(3) Ultimate electrons $\left(n_{e}>10^{9}\right)$, which have energies in the 0.1 to $3 \mathrm{eV}$ range depending on the pressure and local electric fields. The last group 4 is the net result of the energy exchange processes.

The Faraday dark space and positive column ${ }^{5}$ (fig. 5) are nearly fieldfree regions and are characterized by nearly equivalent concentrations of ions and electrons. For these reasons the positive column most nearly resembles a plasma. Unfortunately, in many glow discharge systems the interelectrode separation needs to be small so that the anode is located in the negative glow; therefore, the positive column and the Faraday dark space do not exist. The plasma potential. $v_{p}$, is now essentially the potential of the negative glow, which is the most positive potential anywhere in the system. This potential must be more positive than the next highest potential on any large surface in the discharge by an amount that is at least as great as the first lonization potential of the gas.

Now that an overview of the architecture of dc glow discharges has been presented we can consider a small electrically isolated substrate placed in the glow. Inttially it will be bombarded by electrons and fons, but since electrons have greater velocities than lons the substrate immediately starts to build a negative charge and hence negative potential with respect to the plasma. A negatively charged substrate w1ll repel electrons and attract ions. Thus the electron flux decreases, but the substrate continues to charge negatively until the electron flux is reduced by repulsion fust enough to balance the ion flux. The floating potential, $V_{f}$, is the potential at which equal numbers of electrons and ions arrive at a surface that is not externally blased or grounded.6 The floating potential is related to the electron temperature by the approximate relation given by Chen (1965):

${ }^{4}$ In radiofrequency ( $r f$ ) discharges, the electric fields in the plasma volume may ralse the average electron energy so that secondary and ultimate electron groups are not distinguishable.

5 In a self-maintained discharge the emission is not unlimited so that the plasma column far from the cathode retains a, positive charge. Hence the usage of the term "positive column."

${ }^{6}$ In this case it is the same as the substrate potential, V. 


$$
V_{f}=(1 / 2 e) k T_{e} \ln \left[\pi m_{e} / 2 m_{1}\right]
$$

where

$k$ Boltzmann constant

e unit electron charge

$\mathrm{m}_{\mathrm{e}}$ electron mass

$m_{i}$ ion mass.

In the case of a discharge tube having insulating walls (fig. 5), these walls also require zero steady state net flux, so that wall potential and floating potential are related terms. Since $V_{f}$ is such as to repel electrons, then $v_{f}<v_{p}$. In the absence of a reference, only $v_{p}-v_{f}$ is meaningful; this difference determines the maximum energy with which fons collide with the electrically floating substrate.

\section{Types and Supporting Modes}

In the previous section we have described the classical type of glow discharge which is operated by dc potentials. When an aiternating current (ac) power supply is used (fig. 5), each electrode alternately acts as cathode and anode. On each half cycle a dc-type discharge is established once the breakdown voltage is surpassed. At radiofrequencles ( 5 to $30 \mathrm{MHz}$ ) the electrons oscillating in the glow space have sufficient energies to cause ionizing collisions, thus reducing the dependence of the discharge on secondary electrons and lowering the breakdown voltage. Because rf voltage can be coupled through any kind of impedance it is possible to use reactors without internal electrodes.

Two most common techniques for coupling $r f$ power into a glass tube, of ten referred to as a tubular or tunnel reactor, are shown in figure 6 . In the capacitively coupled reactor $r f$ power is coupled through the capacitance of the dielectric wall of the tube; in the so-called "inductively coupled" reactor, the rf power is also coupled capacitively through the wall, except that coupling is not uniform in that air gaps may be present. Flat-bed reactors ( $1 \mathrm{~g} .7)$, on the other hand, have a configuration in which the substrate rests on a grounded or floating table with the powered rf electrode above. 7 Note that a series capacitor is usually inserted between the $r f$ generator and the powered electrode.

For a tubular reactor the distribution of potential in space taken across the diameter of the tube is symmetrical. In the flat-bed reactor the largest potential difference is found between the plasma and the capacitively coupled electrode, but significant potentials can develop between the plasma and grounded or floating surfaces in contact with the discharge. Koenig and Maissel (1970) have shown that the ratio of these potentials is area dependent. The difference between the plasma potential and that on the powered capacitively coupled surface largely determines how much sputtering of that surface

$7_{A}$ variation of this reactor in which the substrate rests on the powered electrode was used by Holland and 0jha (1976) for deposition of carbon films. 
will occur. Ground potential in flat-bed reactors is always negative with respect to the plasma, so the difference between the plasma potential and ground determines the amount of ion bombardment (sputtering) that occurs on grounded surfaces in contact with the discharge. Vossen (1979) has determined that the plasma potential and floating substrate and wall potentials in a paralle1-plate deposition system and an "inductively coupled" tunnel reactor can vary substantially, depending on the discharge conditions.

Because of the difference in mobility between electrons and ions the static $1-V$ characteristics of the discharge resemble that of a leaky rectifier as shown by Butler and Kino (1963) In figure 8. If an $r f$ signal is applied to this type of load, a very large electron current will flow during one-half of the cycle while a relatively small fon current flows on the other half of the cycle. Since no charge can be transferred through the dielectric wall (or capacitor) between the $r f$ source and the discharge, the electron flow to the surface must decrease. With respect to the applied $r f$ signal this means that the voltage self-biases negatively as shown in figure $8(b)$, until the stable condition, where equal numbers of electrons and lons reach the surface on each half-cycle, is attained. This pulsating negative signal sketched in figure 8 (c) by Anderson et al. (1962) has an average value, $V_{S}$, known as the sheath potential; $\mathrm{eV}_{\mathrm{s}}$ is the average energy with which ions enter the surface sheath on their way to colliding with the surface. Ions can enter the sheath at any time, and so the distribution of ion energies ranges from 0 to almost 2 $\mathrm{eV}_{s}$. It is the negative self-bias that makes the inside walls of tunnel reactors and internal powered electrodes of flat-bed reactors sputtering targets.

There are numerous variants of the basic designs of $r f$ deposition systems described above. We may mention here a system described by Rosler and Engle (1979), which combines the higher throughput of wafers in tunnel reactors with the uniformity of $f 11 \mathrm{~m}$ thickness and properties in parallel-plate reactors. Sokolowski (1979) and Sokolowski et al., (1981) used a high energy pulsed plasma rather than a continuous discharge for avoiding excessive substrate heating. Veprek (1980) has described a reactor in which a material is transported from a solid phase by plasma dissoclated gas, such as hydrogen, and decomposed by thermal and plasma activation in another part of the reactor.

In general most investigators in the field of plasma film deposition have used dc and $r f$ discharges (internally or externally excited). Only few experiments have been reported using microwave discharges. We may cite here the work of Avni et al., (1983), and Shiloh et al.. (1977). Typical microwave generators are magnetron or klystron devices radiating at $2.45 \mathrm{GHz}$. The energy is transferred via waveguides, which are metal tubes, usually of rectangular or circular cross section, or resonant cavities (Evenson, Broida, etc.) which are generally cylindrical in shape (fig. 9). Since the glass discharge tube is usually placed along the axis of the cylindrical cavity, the mode of oscillation of the electromagnetic radiation should be chosen so that the electric field lines near the center are parallel to the cavity's axis. This condition is fulfilled in the $T_{01}$ mode, for which the radius of the cylinder should be selected equal to $0.383 \lambda$ where $\lambda$ is the wavelength of the microwave radiation.

There are basic differences in the fundamental mechanism operating at microwave frequencies. In an alternating field the free electrons oscillate $90^{\circ}$ out of phase with the field and cannot derive power from the fleld (on the 
average). To acquire energy their collisions with neutral molecules must alter their phase relationship with the fleld. If we assume that the excitation frequency $f$ is greater than the elastic collision frequency, the maximum displacement $x$ of an electron due to the high frequency field is given by

$$
x=e E / 2 \pi^{2} m_{e} f^{2}
$$

where $E$ is the fleld strength. In a typical microwave excitation, $E=30 \mathrm{~V}$ $\mathrm{cm}^{-1}$, so that for $f=2.45 \mathrm{GHz}$, substitution in equation (2) gives $x<$ $10^{-3^{\circ}} \mathrm{cm}$. The corresponding maximum electron energy acquired during the cycle is eEx, about $0.02 \mathrm{eV}$. Thus, the electrons slowly accumulate the energy necessary to undergo inelastic, lonizing collisions and to sustain the discharge.

As noted in the Introduction, only a few percent of the gas atoms in a glow discharge are fonized. Varlous conflgurations to enhance ionization are described by Holland (1974). For increasing the ionization efficiency magnetic fields have been interposed. A magnetic fleld normal to the cathode surface constrains secondary electrons to follow a helical, rather than straight-line path. The effect is to give electrons a longer path length for a flxed mean free path, thus increasing the probability of ionizing collisions before the electron reaches the anode. The magnetic field pinches the discharge in toward the center of the cathode, resulting in nonuniformity of film thickness. On the other hand, a magnetic field parallel to the cathode surface can restrain the primary electron motion to the vicinity of the cathode and thereby increase the ionization efficiency. However, the $E X B$ motion causes the discharge to be swept to one side. This difficulty has been overcome by using cylindrical cathodes which allow the $E X$ B drift currents to close on themselves. This is the general magnetron geometry. Remarkable performance is achieved when end losses are eliminated. The first operation in the magnetron mode for sputter deposition was achleved by Penning (1936). Since 1969 considerable development in this fleld of work has taken place and the reader is referred to articles by Thornton and Penfold (1978). Fraser (1978) and Waits (1978). Morosoff et al. (1978) have reported magneticaliy enhanced deposition rates in $\mathrm{rf} g$ low discharges' in ethylene.

Besides magnetic field ionization enhancement may be accomplished by using hot fllament cathodes and hollow cathodes. What is distinctive about the hot fllament discharge is that electron emission from the cathode is primarily by thermionic emission (rather than by ion impact) which can be increased almost indefinitely in the presence of an anode which withdraws the electron space charge surrounding the fllament (usually made of tungsten). In the hollow cathode discharge (von Engel, 1965) a cylindrical cathode is usually used and the ionization is increased by faster electrons from one dark space which enter the other.

\section{Processes Occurring in Discharges}

There are several pathways by which various kinds of particles are produced and react in a glow discharge: Nasser (1971) has given an excellent discussion of the ionization and deionization processes. Kaufman (1969) has reviewed the basic physical processes leading to the production of atoms and simple radicals. Because of the complexity involved in discharge phenomena 
assoclated with polyatomic molecules of the sort used for deposition of films, say $\mathrm{SiH}_{4}$, little or no discussion is to be found in the published literature. Glow discharges in water vapor, carbon dioxide, ammonia and simple hydrocarbons are good examples of the chemical complexity of polyatomic discharge systems (Venugopalan and Jones, 1968; Capitell1 and Molinar1, 1980).

Figure 10 summartzes the many processes occurring in glow discharges. The most important ionization processes are the lonization of gas molecules by electron collision and by absorption of radiation, also known as photoion1zation (Venugopalan, 1971). The uv/visible photons in glow discharges have energies in the range 3 to $40 \mathrm{eV}$ (von Engel, 1965). The formation of negative lons occurs when free electrons are avallable to attach themselves to neutral atoms or molecules, usually one or two electrons deficient in the outer shells. The widely used rf "electrodeless" discharges (fig. 6) are almost similar to the positive column of dc discharges (fig. 5), which has a small and constant axial voltage drop, and only a small imbalance of charge carriers, because, a)though electrons initially diffuse to the tube wall faster than ions, the resultant radial field prevents further charge separation and forces electrons and ions to diffuse equally fast. This process is called ambipolar diffusion and contributes significantly to the disappearance of charged particles.

Because of ambipolar diffusion the currents of electrons and positive fons reaching the wall must be equal:

$$
\begin{aligned}
1_{e} & =-D_{e} \nabla n-n_{\mu_{e}} E_{s} \\
& =-D_{j} \nabla n+n_{\mu j} E_{s} \\
& =1_{1}
\end{aligned}
$$

where $n=n_{e}=n_{1}$ is plasma density, on the density gradient, $D$ the diffusion coefficient, $\mu$ the moblitity and $E_{S}$, the fleld caused by the (sma11) space charge. By eliminating $E_{s}$ in equation (3), it is seen that

$$
\begin{aligned}
\mathcal{I} & =-\left[\left(D_{e} \mu_{\mathcal{1}}+D_{j} \mu_{e}\right) /\left(\mu_{\mathcal{1}}+\mu_{e}\right)\right] \nabla n \\
& =D_{a} \nabla n
\end{aligned}
$$

This is the defining equation for the ambipolar diffusion coefficient, $D_{a}$.

The disappearance of charged species by ambipolar diffusion in the absence of a source term is given by the diffusion equation:

$$
\delta n / \delta t=D_{a} \nabla^{2} n
$$

By solving the equation for the case of an infinite cylinder it is seen that the diffusion-controlled loss under steady-state conditions with a spatially well distributed source term is closely approximated by a first-order rate constant

$$
\mathrm{k} / \mathrm{s}^{-1}=5.78 \mathrm{Da}_{\mathrm{a}} / \mathrm{r}_{\mathrm{o}}^{2}
$$


In a plasma deposition reactor using a tube $1 \mathrm{~cm}$ in diameter, equation (6) will yield $k=1 \times 10^{5} \mathrm{~s}^{-1}$, so that electron impact ionization is the major source term for charged species in the discharge. When negative fons are also present, as for example in glow discharges of electro-negative gases such as $\mathrm{SF}_{6}$. their principal effect is to accelerate the ambipolar diffusion of the electrons.

If Penning mechanism which involves direct lonization by collision with metastables were to occur, the ionization energy of the metastable must exceed the lonization potential of the other reactant. Typical energies of metastables are in the range 0 to $20 \mathrm{eV}$ and are to be compared with the energies of glow discharge electrons $(0$ to $20 \mathrm{eV})$ and lons $(0$ to $2 \mathrm{eV})$.

Several radlative, two-body, and three-body recombination mechanisms are recognized for the disappearance of the charged particles. Of these, the fast reactions are the dissoctative recombination of electrons and positive molecular tons

$$
A B^{+}+e^{-}=\dot{A}+\dot{B}
$$

where the products may be electronicaliy excited atoms or free radicals. The generaliy observed range for the rate constant of this type of reaction is 1 to $5 \times 10^{-7} \mathrm{~cm}^{3}$ molecule $\mathrm{m}^{-1}$. Such products are also formed by the dissociation decay of molecules excited by electron impact:

$$
\mathrm{e}^{-}+A B \rightarrow \mathrm{e}^{-}+(A B)^{\star} \rightarrow \mathrm{e}^{-}+\mathrm{A}+\mathrm{B}
$$

For this event to occur the energy of the electrons must exceed the dissociation energy of the molecule; the transition must occur within the limits set by the Franck-Condon principle. The energies avallable in many glow discharges ( 0 to $20 \mathrm{eV}$ ) favor dissociation by electron impact via excited states.

Electron impact excitation of vibrational energy is a highly specific process the cross section of which can differ by two orders of magnitude from one molecule to another. The dissociation will usually come about via the repulsive upper state which dissociates upon its first pseudovibration, 1.e., in about $10^{-14} \mathrm{~s}$ (fig. $11(\mathrm{a})$ ). If the upper state is bound, but the molecule is on the repulsive part of its potential energy curve at a point above its dissociation energy (fig. $j($ (b)). dissoclation will occur on its first vibration, also in about $10^{-13} \mathrm{~s}$. Excitation to stable excited state followed by a shift to an unstable excited state ( $f 1 g .11(\mathrm{c})$ ) gives rise to a condition known as predissoclation. Depending on the degree of mixing of states, this predissociation process is much slower than the other two processes. The overall cross section and rate constant for dissociation will normaliy equal that of the primary excitation step. That is to say, there will be near unit probability for the subsequent dissociation, because collisional or radiative ilfetimes of the excited states cannot be shorter than about $10^{-7}$ to $10^{-8} \mathrm{~s}$.

Dissoclative attachment reactions such as

$$
e^{-}+A B \rightarrow A^{-}+B
$$


have rate constants in the range of $10^{-12}$ to $10^{-11} \mathrm{~cm}$ molecule $^{-1}$ $s^{-1}$, and are considered less important sources of atoms and free radicals. Further, many associative detachment reactions such as

$$
A^{-}+B \rightarrow A B+e^{-}
$$

have rate constants of 1 to $5 \times 10^{-10} \mathrm{~cm}^{3}$ molecule $\mathrm{e}^{-1} \mathrm{~s}^{-1}$ so that negative ions are unlikely to be of importance in rapidly pumped steady-state glow discharges. Two-body ion-ton recombinations have rate constants in the same range as reaction (7) and must be included particularly in glow discharge systems of electron attaching gases.

Because of strong ion-dipole and ion-induced dipole interactions charge transfer and ion-molecule reactions are important:

$$
\begin{aligned}
& A^{ \pm}+B C \rightarrow A+B C^{ \pm} \\
& A^{ \pm}+B C \rightarrow A B^{ \pm}+C
\end{aligned}
$$

If the reactions are exothermic they have small or no activation energy; Frequently, their rate constants are of the order of $10^{-9} \mathrm{~cm}^{3}$ molecule $\mathrm{e}^{-1}$ $s^{-1}$ and will therefore go to completion in a small fraction of the residence time of the gas in the glow discharge.

From the standpoint of surface chemistry it is necessary to consider catalytic effects of which little is known. As Venugopalan and Veprek (1983) pointed out the lack of understanding of adsorption of discharge products by reactor walls and the catalysis of reactions by these walls is a major problem that is associated with all plasma deposition systems. Ion-impact induced fragmentation of physi-sorbed molecular layers followed by chemisorption of the fragments and subsequent reaction may lead to deposition. The activation of the gas phase enhances reactions such as

$$
A(s)+(m / n) B_{n}(g) \rightarrow A B_{m}(s)
$$

whenever the dissociative chemisorption of the species $B_{n}$ on the $A B_{m}-$ surface is the rate-determining step. When high species fluxes impinge on the surface the rate determining step becomes the bulk diffusion. The deposition (evaporation, etching, sputtering, etc.) process

$$
a A(s)+(b / n) B_{n}(g) \rightarrow A_{a} B_{b}(g)
$$

can be catalyzed either via formation of reactive radicals, e.g., atoms, in the plasma phase or by bombardment of the surface with ions or electrons possessing sufficient energy. A number of these processes have been reviewed by Winters (1980).

The review of results to be presented later will consider many of these iprocesses in important glow discharge deposition systems.

\section{Problems Under Film Deposition Conditions}

From the standpoint of reactor design and physical effects of glow discharges, there are several other processes which are essentially the same 
as plasma deposition. For example, glow discharge polymerization and etching uses equipment similar to that used for plasma deposition. In plasma polymerization the starting gas is a monomer, which, when decomposed by the glow discharge, forms spectes that polymerize into an organic film. In plasma etching the gases used are selected for their ability to produce reactive species upon decomposition, which react chemically with a surface to be etched to form gaseous components that are easlly pumped away. With fluorocarbons both polymerization and etching may occur, as indeed Kay et al.. (1980) have demonstrated, depending on slight perturbations in the system. The kinetic and mechanistic aspects of these processes have been reviewed by a number of authors, among them we may cite Bell (1980), 'Carml et a1., (1981), Coburn (1982), Flamm and Donnelly (1981), and Vinogradov et al., (1982).

There are as many reactor designs and experimental conditions to consider as there are published studies on plasma deposition. Nevertheless, for understanding the complexities involved in $f 11 \mathrm{~m}$ formation it is sufficient to take a close look at the deposition region. This is usually the substrate area which is subjected not only to plasma radiation but also to fluxes of energetic and reactive, charged and neutral particles in various excited states. Figure 12 shows the luminous region around a graphite substrate which is (a) floated; (b) grounded, or (c) biased at $-100 \mathrm{~V}$ in a 16 percent $\mathrm{C}_{3} \mathrm{H}_{6}+\mathrm{Ar}$ rf induction plasma under otherwise the same experimental conditions. It is obvious that the width of the luminous region, which we shall call the plasma layer (PL), is the largest for the biased substrate and the smallest for the floated substrate. The PL wraparound the grounded and the negatively blased substrate (even in the direction opposing the gas flow) while on the floated substrate the PL is formed onty on the surface facing the direction of the gas flow. Further, the PL luminosity is often very much higher than that of the plasma bulk (PB) suggesting that there is a surface neutralization/recombination layer (NL) interposed between the substrate bulk (SB) and the PL. These regions are delineated in figure 13.

Since electrons are repelled by the potential difference $v_{p}-V_{f}$, it follows that the substrate w11l acquire a positive space charge sheath around it. According to Bohm (1949), between this sheath and the PB there is a quast-neutral transition region of low electric fleld, the effect of which is to increase the velocity of the positive ions to enable them to penetrate the sheath and bombard the substrate. Hagstrum (1961) has shown that the probability of an ion being neutralized upon collision with a surface is greater than 99 percent for a number of lons over a range of kinetic energies. Therefore, it is reasonable to conclude that the fon flux, jis, reaching the substrate surface in a deposition experiment will be neutraijized and adsorbed in the NL. Khait et al., (1980) describe the neutralization of positive ions as short-lived hot spots (SLHS) with a lifetime of the order of 10-14 s. Fluxes of excited neutrals such as metastables, $J_{m s}$, and radicals, $f_{r s}$, will also reach the surface, release their energy, and become adsorbed or trapped in the NL.

- Following Hagstrum (1977) the possible mechanisms for an ion or exclted particle to neutralize at substrate (metal) surfaces involve deexcitation (1) 
by emission of radiation, (2) by an Auger process $s^{8}$ followed by emission of secondary electrons, and (3) by a resonance process whereby an electron is transferred from the conductance band of the metal to an equivalent energy level in the incoming ion or a similar transition where the electron goes from the ion to the metal. Secondary electron emission from the substrate surface back into the plasma bulk takes place by a potential ejection which depends on the ionization potential of the incident ion, and/or by a kinetic ejection which depends on the energy of the particle projectile. The loss of energy of the excited particle to the lattice upon impact is a momentum transfer process which sets the atoms in motion by a process known as "collision cascade". Although a binary collision approximation qualitatively describes the path and energy loss of the incident particle, the consequences of the collision cascade are many and varied: sputtering, backscattering, lattice damage, and the formation of chemical potential gradients ( $\mathrm{flg}$. 13). This last may enhance diffusion and segregation of atoms from the substrate bulk to the neutralization layer and thus modify the surface chemistry. Numerous studies by Gruen et a1., (1983) have demonstrated Gibbsian-type segregation on metallic surfaces.

The yield (potential + kinetic) of secondary electrons $\left(\gamma_{1}, Y_{m}\right.$, and $\mathrm{Yr}$ ) is inversely proportional to the mass but only slightly dependent on the energy of the incoming projectile. It, however, varies depending on the condition of the surface, and is generally lower for nonmetals (higher work function) than for metals. For $600 \mathrm{eV} \mathrm{Ar}+$ ions Chapman (1980) gives

$$
Y_{1}=a \cdot 10^{-2}
$$

with $a<10$ for nonmetals and $a \geq 10$ for metals. According to Kaminski (1965) $\gamma_{m} \approx \gamma_{1}$ for metals, again with Ar as the incoming projectile.

The secondary electrons travelling the sheath along $z$ (normal to the substrate surface) gain energy from the high electric field $E_{s}$ and have an energy distribution function $F_{e s}\left(c_{e s}\right)$ that is anisotropic along $z$ with a maximum around $100 \mathrm{eV}$. Figure 14 shows the values calculated by khatt et a)., (1980) for the field and particle energies in the sheath as a function of gas pressure in a system with a grounded graphite substrate. This high energy electron beam (HEEB), with average energy $\bar{\varepsilon}_{\text {es }}$ and flux fes collides with the particles populating the plasma bulk and loses its energy along $z$. The inelastic collisions with atoms and molecules will result in excitation and ionization and a decrease in HEEB energy to the average value for $P B$ electrons, $\bar{\varepsilon}$ PB. Thus a boundary condition can be set along $z$ between PL and PB:

${ }^{8}$ The Auger effect is the emission of a second electron after high energy radiation or particle has expelled another. Its mechanism is that the first electron leaves a hole in a low-lying orbital which a higher energy 'electron drops into. The energy this releases may result either in the generation of radiation (which gives $X$-ray fluorescence) or in the ejection of another electron, the "secondary" electron of the Auger process. 


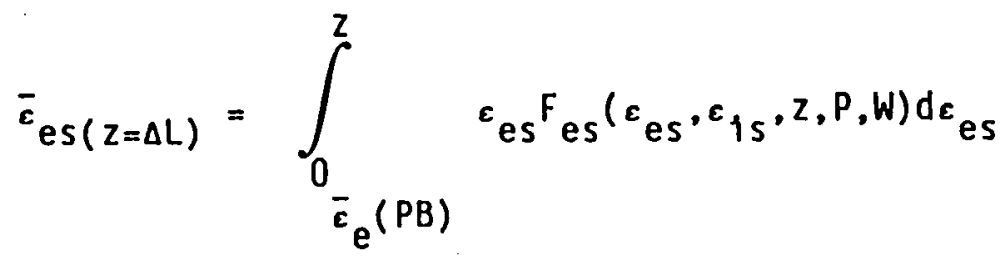

where

$$
\begin{aligned}
& \Delta L \text { thickness of } P L \\
& P \text { gas pressure } \\
& W \text { net power input. }
\end{aligned}
$$

In what follows we shall consider the evidence from work of Khait et al., $(1980,1983)$ to show that the contribution of HEEB electrons to excitation and ionization in PL is significantly greater than that of $\mathrm{PB}$ electrons in PL.

For the ionization process, by analogy with known phenomena produced by the PB electrons, we write:

$$
d n_{j} / d t=\operatorname{sjes} q_{e s}\left[1+\left(a_{j} n^{2} k_{j} \Delta L / j_{e s} q_{e s}\right)\right]
$$

where
$s$ cross section of $\mathrm{PL}$
$q_{e s}$ efficiency of tonization by HEEB electrons
$a_{1}$ degree of ionization $\left(n_{e}=n_{j}=a_{1} n\right)$ in $P B$
$k_{i}$ rate coefficient of lonization in $\mathrm{PB}$
n plasma density.

Figure 15 is a plot of the ratio $\left(d n_{j} / d t\right) P L /\left(d n_{j} / d t\right) P B$ as a function of gas pressure. It is obvious that the contribution by secondary electrons to the lonization in $P B$ is very much greater than the earlier estimates (10 percent) made by Chapman (1980) and winters (1980).

The fraction $A$ of the electron distribution function of HEEB performing particle excitation to a higher quantum level with a cross section $\sigma^{\star}\left(\bar{\varepsilon}_{e s}\right)$ is given by

$$
A(H E E B, Z=\Delta L)=\left[2 \gamma \bar{u}(P B)^{\sigma^{*} \varepsilon} e(P B) / \bar{u}_{e} \sigma^{\star} \bar{\varepsilon} e s\right] B(P B)
$$

where

$$
\begin{aligned}
& \text { B fraction of } \mathrm{PB} \text { electrons causing excitation in } \mathrm{PL} \\
& Y \ll 1 \text { secondary electron coefficient } \\
& \bar{u}_{e} \text { and } \bar{u}_{P B} \text { average velocities of } H E E B \text { and } P B \text { electrons, } \\
& \text { respectively, in } \mathrm{PL} \text {. }
\end{aligned}
$$

Figure 16 shows the spectral 1ine/band intensitles ratio between $\mathrm{PL}$ and $\mathrm{PB}$, .1.e.. I $I_{P L} / I_{P B}$ as a function of gas pressure. These measurements were made in a microwave cavity excited plasma using graphite and silicon substrates, grounded or blased with $-100 \mathrm{~V}$, in flowing pure Ar or mixtures of Ar with 5 vol $\%$ of $\mathrm{N}_{2}$ or $\mathrm{H}_{2}$. The increase in the ratio of $\mathrm{Ar}, \mathrm{N}_{2}, \mathrm{CN}$ and integral intensity (as well as for $\mathrm{CH}$, and $\mathrm{C}_{2}$ not shown) with increasing gas pressure suggests that decreasing the mean free path rather than $\Delta L$ causes more collisions and results in a higher population of the excited states for 
the various species. The correlation between the ratio of $\mathrm{SiH}$ and $H_{\alpha}$, and of $\mathrm{CN}$ and $\mathrm{N}_{2}^{+}$, on the other hand, indicates the interaction of excited positive ions and radicals with substrate surface and/or the sputtered away particles $S i$ and $C$, respectively.

By neglecting elastic collisions, Khait et al.. (1983) approximated the inelastic collisions causing excitation and ionization to the total number $\alpha$ of collisions with gas particles. In each a collision the HEEB electrons undergo scattering and their motion can be described by a Brownian motion with a drift velocity $v_{\alpha}$ which decreases rapidly along $z$ towards PB. Accordingly, the thickness $\Delta L$ of $P L$ is estimated as:

$$
\begin{aligned}
& \Delta L=\alpha^{1 / 2}\left\langle\lambda_{H E E B}\right\rangle
\end{aligned}
$$

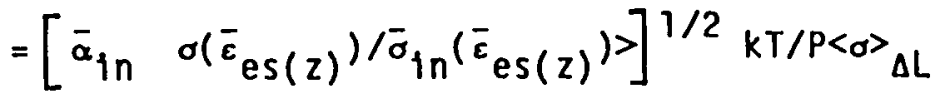

where

o total collision cross section

$\bar{\sigma}_{\text {in }}$ average value of the cross section for inelastic collisions

$\bar{\alpha}_{\text {in }}$ number of inelastic collisions, and

$$
\lambda_{\text {HEEB }}=1 / n_{\sigma}=k T / P_{\sigma}
$$

is the mean free path of HEEB electron in PL at plasma gas temperature $T$ and total pressure P. The same authors (1980) considered the electron trajectory $b$ due to elastic and inelastic collisions of the HEEB's electrons in PL and evaluated

$$
\Delta L=b / n o
$$

having defined

$$
b=\left(\left[\left(\varepsilon_{\text {es }}-\bar{\varepsilon}_{\text {es }}(\mathrm{z}=\Delta L)\right) / \delta \bar{\varepsilon}_{\text {in }}\right]<\sigma / \sigma_{\text {ion }}+\sigma_{\text {ex }}>\right)^{1 / 2}
$$

where

$\begin{aligned} \delta \bar{\varepsilon}_{1 n}= & <\delta \varepsilon i o n+\delta \varepsilon e x_{\Delta L} \text { is the energy lost by the average HEEB } \\ & \text { electrons in inelastic collisions for ionfzation (ion) and }\end{aligned}$ electrons in inelastic collisions for ionfzation (fon) and
excitation (ex) in PL, while ofon and oex are the inelastic collision cross sections resulting in ionization and excitation, respectively. By combining equations (19), (21), and (22) we finally get the following relationship for the thickness of PL:

$$
\Delta L=\left(\left[\left(\varepsilon_{e s}-\bar{\varepsilon}_{e s}(Z=\Delta L)\right) / \delta \bar{\varepsilon}_{\text {in }}\right]<\sigma / \sigma_{\text {ion }}+\sigma_{e x}>\right) 1 / 2 k T / P<\sigma>\Delta L
$$

Note that the thickness of $\mathrm{PL}$ is dependent on $\mathrm{P}, \mathrm{T}$, the nature of the gas particles and the power input into the plasma. Figure 17 shows that values of $\Delta L$ measured by optical emission of the excited gas and sputtered particles in PL are in reasonable agreement with the calculated values. 
Besides secondary electrons there are other particles released from NL into PL, such as reflected particles, Jrefl, sputtered atoms, Jsputt, and chemically etched molecules, Jetch (fig. 13). As previously noted, the sputtering and etching phenomena influence film deposition. Sputtering experiments can provide information on the thickness of $\mathrm{NL}$. In reviewing the work of a number of authors Winters (1980) concluded that the thickness of NL is about $30 \AA$. Naturally, this w111 depend on the mass, energy and angle of impact of the incident ion as well as on the surface conditions, like crystal lattice and surface coverage, and type of substrate (polycrystalline, amorphous, metallic or semiconductor, etc.). If we sum all lons arriving from $P B$ and formed in PL by interaction with HEEB electrons we get for the incident flux of ions

$$
j_{1 s}=\frac{1}{4} \sum a_{1} u_{1}
$$

Their maximum energy, $\varepsilon$ is, depends on $v_{p}-V_{s}$ or $v_{p}-V_{S}$, where $v_{S}$ and $V_{S}$ are the sheath and substrate potentials, respectively, and is a function of

$$
\varepsilon_{1 s} \propto f\left[\left(k T_{e} / m_{1}\right)^{1 / 2} ; P, W, E_{S}, F_{e s}\left(\varepsilon_{e s}\right), V_{S}, V_{S}\right]
$$

In glow discharges, PL usualiy tends to follow the contour of the surface and therefore most fons approach the surface at normal incidence.

The sputtering yield, which is defined as the number of substrate (target) atoms ejected per incident lorr, has been determined for numerous metals and a variety of ions with different energies. The interested reader is referred to tabulations by Laegried and Wehner (1961), Kaminski (1965), Vossen and Cuomo (1978), Chapman (1980), Roth (1982), and Thornton (1982). We have collated few data in table I for those elements that may be encountered in one glow discharge deposition system or another. These data suggest that sputtered particles with a flux 0.1 to 3 times that of incident ions ( $n_{j}=10^{11}$ $\mathrm{cm}^{-3}$ ) will enter PL where they will undergo excitation and/or ionization by interacting with PB and HEEB electrons. Therefore, some of the sputtered particles will return to the surface as ions and enhance its chemistry, for example, as in ion-nitriding, boridizing and carburizing processes.

Some of the sputtered particles will contribute to cluster formation in PL (fig. 13). Table II gives data for the degree of ionization and the normalized amount of polymerized species in both PB and PL (around a grounded graphite substrate). In comparison with PB there is a higher concentration of positive ions $\left(n_{1}\right)$ and excited polymerized ( $n_{p o l y}$ ) molecules in PL because of the interaction of sputtered particles with HEEB electrons. Along $z$ axis, towards the substrate surface, where a large number of collistons with HEEB electrons take place, $n_{1}$ and $n_{\text {poly }}$ increase to such magnitudes, that the supersaturation condition described by Lothe and Pound (1969) for the formation of clusters is established. Phenomenologically, we may consider two processes along the $z$ axis from PL towards the substrate surface:

(1) The fonization rate due to HEEB electrons interaction is higher near the sheath than at the boundary between PL and PB. Near the sheath the HEEB electrons reach their maximum energy ( $\mathrm{fig} .17)$. The ions thus formed with a long range dipole moment attract and attach polymerized molecules such that 
polymer clusters are formed. Evidence for "prenucleation" near the surface has been found in experiments using focused uv lasers by Ehrilich et al., (1981), Brueck and Ehrlich (1982), and Ehrlich and Tsao (1983).

(2) Polymerized species in the PL are in a three-coordinate system, but they lose one coordinate upon reaching the surface and must remain on the solid in a two-coordinate system. As de Boer (1969) pointed out this increases their concentration as well as the probabllity for clustering and nucleation.

Thus we see that many of the particles coming towards the surface react with the substrate, perhaps by chemical bonding, and form diffusion-controlled layers or deposits of fllms in NL. Ion-enhanced dry chemical etching takes place in the opposite direction, particularly in systems containing hydrogen and halogens. The etching process returns species deposited on the surface back to PL at rates which depend upon etch parameters described by Flamm and Donnelly (1983) and Coburn (1982). Both processes proceed simultaneously; depending on their rates deposition or etching will result. Apparentiy, under f17m formation conditions deposition prevalls because of a higher rate of deposition than etching. The fluxes of lons, metastables and radicals are continuous, as are the sputtering and etching losses during deposition. Consequentiy, we may expect the material balance in the film to change with deposition time.

In summary, the "dialogue" between the plasma of a glow discharge and the substrate immersed in it involves the following processes (fig. 13):

A. Impact of positive ions, excited radicals and metastable states with the grounded or negatively blased substrate surface.

B. Neutralization, momentum transfer, trapping, adsorption, and incorporation of the above species in the surface resulting with the formation of NL in which, due to a gradient of chemical potentlal and cascade mixing. diffusion and segregation of particles from NL to SB and vice-versa occurs.

C. Emission of secondary electrons (HEEB), sputtered particles and chemically etched species from NL back to the plasma forming the PL.

D. The HEEB electrons enhance excitation and ionization processes in $\mathrm{PL}$. As a result cluster formation and nucleation takes place leading to film deposition.

\section{DIAGNOSTICS: THEORY AND EXPERIMENT}

The history of homogeneous reactions in the PB is transmitted along to the heterogeneous plasma - surface interactions controlling the film deposition processes. This continuity approach is schematically shown in figure 18. The monomer - carrier gases or vapors fed to the plasmas are partly excited, $k_{e x}$, and ionized, $k_{1}$, by energetic electrons; interaction with fresh monomer molecules results in its dissociation, $k_{D}$, and fragmentation, $k_{F}$, reaction rates. The homogeneous reactions with fresh monomer molecules continues with the formation of new polymeric species with kpoly reaction rates. Transported by convection, diffusion, electric fields and the flow velocity in the plasma system these new particles reach the substrate surface 
region. The kinetic mechanisms and the rate determining step of monomer dissociation and formation of polymeric species are the link between the PB homogeneous reactions and the plasma - surface heterogeneous reactions.

Methods for determining the temperature, density and composition of plasmas are the subject of an important part of experimental plasma physics which has come to be called "dlagnostics." Many physical phenomena occurring in plasmas can be utilized to provide diagnostics. For example, the intensity and spectral composition of radiation emitted by a plasma depends on the electron energy, its distribution function and on the plasma density. With proper care, the electron density, its temperature and energy distribution in a plasma can be determined simultaneously by an electrostatic (Langmuir) probe. Methods such as mass spectrometry and electron paramagnetic resonance can provide information on atomic and molecular, ionic and free radical species in the plasma. The interpretation of these measurements involves theories with limiting assumptions. The literature is abundant with general as well as speclalized treatises on plasma diagnostics, for example, by Huddlestone and Leonard (1965), Lochte-Holtgreven (1968), Podgornyt (1971), Venugopalan (1971), and Eubank and Sindoni (1975).

The confident characterization of a given plasma generally requires that several measurement methods give a consistent picture. Unfortunately, we find, particularly in the early publications, only rarely the application of even one dlagnostic tool in film deposition studies. Many recent works, however, use a combination of methods such as probes and mass spectrometry, and occasionally includes emission spectroscopy. Even then it is difficult to assess whether proper care has been exercised in the measurement and interpretation. one must know the fluxes and energles of lons and electrons incident usualiy on the substrate surface. Because the energy of positive ions incident on surfaces is determined by $v_{p}-v_{s}$, it is necessary to know these potentials as well. Even if spatially resolved measurements are made from as close a position as the substrate surface to the other end of the glow the fluxes thus obtained will include for a given specles not only those responsible for film deposition but also those that are etched and sputtered in deposition processes. In those cases where deposition has been reported outside the glow region it is also necessary to perform diagnostics of the afterglow and sometimes even the foreglow regions. While such cases have resulted in good quality fllms occasionally, clearly due to the absence of positive ion bombardment, these are more often the result of poor apparatus geometry or configuration and parameter control. The following discussion of theory and experiment is directed at some of these problems as we 11 .

\section{Spectroscopic Methods}

Spectroscopic plasma diagnostics can give information about the concentration of various species and their temperatures without disturbing the plasma. The simplest technique is the direct analysis of the plasma emission -using a spectrograph with adequate resolution. Line radiation in the visible and ir spectra is useful for identffying excited species. Relative line intensities give the temporal and spatial variations of species in the $P B$ and $\mathrm{PL}$. Under some conditions it is possible to relate absolute line intensity measurements to the density of energetic electrons. Absorption measurements using suitable radiation source can be used to determine the populations of 
nonradiating metastable levels or first-excited states that radiate in the vacuum uv. Both emission and absorption spectroscopies are nonintrusive in situ techniques for investigating and monitoring the complex processes occurring in the glow discharge $\mathrm{PB}$ and $\mathrm{PL}$. In the following sections we shall brlefly survey the theory and experiment involved in these methods of diagnostics. We shall also review the more recent methods of laser-induced fluorescence spectroscopy and anti-Stokes Raman scattering spectroscopy.

Emission spectroscopy. - The radiation emitted from a glow discharge may exhibit a variety of spectra as indicated in table III, which is taken from Cabannes and chapelle (1971). Its analysis by spectroscopic measurements of continuum intensities, line intensities, line profile, etc. is the basis of the diagnostic technique of emission spectroscopy. Aithough a wide range of frequencies is emitted it is the optical region that has been extensively studied. The optical radiation is due to radiation arising from the capture of free electrons into various excited states of the atoms and ions and from the acceleration of electrons (bremsstrahlung) during collisions with fons or atoms. Its interpretation is based on the assumption of an electron distribution determined exclusively by particle collision processes.

The glow discharges used in $\mathrm{f} 11 \mathrm{~m}$ deposition operate at gas pressures in the range of $10^{-2}$ to 10 torr, meaning that local thermal equilibrium (LTE) conditions do not prevali in the plasma. The temperature values of the electrons $\left(T_{e}\right)$ are much higher than its values for excitation $\left(T_{e x}\right)$, ionization $\left(T_{f}\right)$ or translation ( $\left.T_{\text {gas }}\right)$. Under such conditions the absolute or relative spectral ine intensities for the evaluation of $T_{e x}$ and $T_{1}$ should be treated with great care. The same care has to be applied in measuring the spectral line profile for Stark, Doppler, or pressure broadenings ( $n_{e}, T_{e}$, and $T_{g a s}$ values, respectively). At low pressures, where plasma density $\mathrm{n}_{\mathrm{e}} \geq 10^{12} \mathrm{~cm}^{-3}$, the normal procedures of Stark broadenings for $\mathrm{H}$ or $\mathrm{He}$ line spectra which are valid for $\mathrm{n}_{\mathrm{e}} \geq 10^{15} \mathrm{~cm}^{-3}$ cannot be used (see Griem (1964) and Lochte-Holtgreven (1968)). Instead spectral 1 ines with high quantum number may be used, for example, $H_{n}>10$ in the hydrogen Balmer sertes as indicated by Bergsted et al. (1962).

The experimental techniques have been described by a number of authors. Griem (1964), Turner (1965), Kimmitt et al. (1965), Lochte-Holtgreven and Richter (1968) are excellent sources of information. Greene and Sequedaosorto (1973) and Greene (1978) describe experimental set-ups suitable for glow-discharge sputter deposition systems; Harshbarger et al. (1977, 1978) describe geometries conventent for plasma etching; and Matsuda et al. (1980), Kampas and Griffith (1981), Tanigucht et a1. (1980), Inspektor et a1. (1981), Perrin and Delafosse (1980), Knights et al. (1982) and many others describe apparatus for glow discharge decomposition systems which deposit films. Apparatus for a typical glow discharge optical spectroscopy (GDOS) work is shown in figure 19. A series of silts placed along the optical axis between the discharge reactor and the spectrometer are used to focus the spectrometer on a narrow region of the glow along the reactor axis. The optical windows should be placed as far from the discharge as possible to minimize loss of transmission by film deposition. With adequate design it is quite possible to obtain intensity versus position profiles in the reactor. In the beli-jar systems described originally by Bradley and Hammes (1963) and modified by several others, for example, Williams and Hayes (1967), optics extending through the base plate may be used to achieve the same results. Either dc or 
ac optical detection schemes may be employed. In case where maximum sensitivity is desired, pulse counting techniques are preferable to minimize random electronic noise from the photomultiplier tube.

The emission intensity at any position $x$ in the discharge for a given transition is governed by the excitation efficiency or probability which depends on $n_{e}$ and $f(E)$, and is generally unknown for the complex species encountered in many deposition systems. Therefore, for quantitative diagnostic information it is necessary to calibrate the signal intensity with standard samples under known and comparable discharge conditions. While this can be done for the stable molecular species without much difficulty, it is impossible with reactive intermediates such as free radicals. Consequently, in most instances the optical emission from deposition plasmas is monitored for the presence or absence of lines to signify the spatial distribution of spectes of interest for several plasma parameters. For this last the following approach described by d'Agostino et a1. (1981) is typical.

If the direct electron impact from the ground to the emitting state is by far the most important excitation process we can write

$$
\begin{aligned}
& e+x \stackrel{k e}{\longrightarrow} x^{\star}+e \\
& x^{\star} \stackrel{k}{\rightarrow} x+h v \\
& x^{\star}+M \stackrel{k_{Q}}{\longrightarrow} x+M
\end{aligned}
$$

where

$x^{*}$ emitting state

$X$ any lower state including the ground state.

The $k$ 's represent the approprlate rate constants for the direct electron impact excitation process $\left(k_{e}\right)$, the radlative process $\left(k_{R}=1 / \tau\right.$, where $\tau$ is the natural lifetime), and for collisional deactivation $\left(k_{Q}\right)$. At steady state, the intensity of optical emission is given by

$$
I_{X} \propto k_{e} n_{e}[X] /\left(1+[M] k_{Q} / k_{R}\right)=C_{n_{X}}[X]
$$

The equality is obtained by assuming $[M] k_{0} / k_{R}$, which is generaliy less than unity, to be a constant at constant pressure. The excitation efficiency $n_{x}=k_{e} n_{e}$ is clearly a function of the density and energy distribution function of the electrons, which are all related to the discharge parameters.

Inert gas "discharge" actinometry. - The possibility of using an actinometric technique for plasma diagnostics was suggested by Kaufman (1976).

- Since then increasing attention has been paid to a technique that has come to be known as inert gas (discharge) actinometry.

Coburn and Chen (1980) added a small amount of Ar to a reactive plasma and monitored the Ar emissions concurrentiy with those of the reactive particle $(X)$ :

$$
\begin{aligned}
I_{A r} & =k_{A r} n_{A r} n_{A r} \\
I_{X} & =k_{X} n_{X} n_{X}
\end{aligned}
$$


where $k_{A r}$ and $k_{X}$ are proportionality constants. Since $n_{A r}$ is known, the excitation efficiency, nar. of any of its levels is determined simply by $I_{A r} / n_{A r}$. If the excited state responsible for the $\operatorname{Ar}(\sim 13.5$ eV) optical emission matches closely in energy with the level responsible for an emission line from a reactive species ( $F \sim 14.5 \mathrm{eV})$, then the same group of electrons will be responsible for the excitation of both levels.. The excitation efficiencies of these levels of $\mathrm{Ar}$ and the reactive particle will then have similar dependence on glow discharge parameters and we may assume $n_{x}=$ $\mathrm{kn}_{\mathrm{Ar}}$ as discharge parameters are varied. Thus, the relative changes in the reactive particle density can be determined by combining its emission intensity with the excitation efficiency of Ar:

$$
n_{X} / n_{A} r=K I_{X} / I_{A r}
$$

where $K$ is a constant independent of the discharge parameters. Coburn and chen tested the technique indirectly in a $\mathrm{CF}_{4}+\mathrm{O}_{2}$ system by establishing a correlation between $F$ atom emission intensities, normalized to $\mathrm{Ar}$ emission intensities, and $F$ atom concentrations measured downstream of the discharge by chemical titration.

Similar work was subsequently reported by d'Agostino et al. (1981 a,b, 1983) who used both $\mathrm{Ar}$ and $\mathrm{N}_{2}$ as inert gas actinometers in $\mathrm{CF}_{4}+\mathrm{O}_{2}$ and. $\mathrm{SF}_{6}+\mathrm{O}_{2}$ glow discharges, by Tiller et al. (1981) who used $\mathrm{Ar}^{2}$ in $\mathrm{CCl}_{4}$ discharges, and Ibbotson et al. (1983) who used $\mathrm{Ar}$ in $\mathrm{Br}_{2}$ discharges. Ibbotson et al. (1983) in a direct test found that normalized Br atoms emission, from a Br 2 plasma with an Ar actinometer, correlates with Br atom concentration determined by in sttu $\mathrm{Br}_{2}$ optical absorption. However, recently Donnelly and Flamm (1983) have obtained time-resolved emission spectra which suggest that $\mathrm{Cl}$ emission, normalized to $\mathrm{Ar}$, from a $\mathrm{Cl}_{2}$ discharge may not be a reliable measure of the instantaneous $\mathrm{Cl}$ atom density. Further work which will establish directly and unambiguously the regions of validity is called for before actinometry is widely employed for diagnostic purposes.

Emission spectroscopy was used to measure the relative intensity ratio of same spectral line and band in the $P L$ and in the $P B\left(I_{P L} / I_{P B}\right.$ ) as shown in figure 16. The $I_{P L} / I_{P B}$ ratio allows the correlation between the projectile particles to and the sputtered particles from the surface as function of rf power input as shown in figure 20 for $H_{\alpha}$ and SiH on a single Si crystal and for $\mathrm{N}_{2}{ }^{+}$and $\mathrm{CN}$ on a graphite substrate. The relative spectral intensity was used for scanning the emission spatial distribution in the PL. Selwyn and Kay (1983) reported the use of optical fibers for spatially resolving the emission of fluorocarbons in rf plasmas.

Absorption spectroscopy. - The major drawback of emission spectroscopy is that information is obtained only about species which are already excited. This may often represent only a very small proportion of the total number of the species in question. Absorption spectroscopy would be advantageous here, but higher resolution is then required because with low resolution sharp lines are readily lost in the background. Another difficulty is the possible absence of active species (transients) in concentrations sufficient to give rise to detectable absorption in easily accessible regions of the optical spectrum. Nevertheless, with high resolution the relative populations in the different rotational and vibrational levels can be deduced. It should be noted that the intensities of interest may often be modified by absorption due to other parts of the species, or by overtones and combination bands. 
In a typical experimental set-up a lamp or laser beam which emits the appropriate frequency is used as the radiation source (fig. 18). In sputterdeposition systems a hollow-cathode lamp containing the element of interest is desirable. The light beam is modulated and the absorbance of the discharge is determined using a phase sensitive lock-in detector tuned in synchronous to the chopper frequency. Tuned ac detection, of course, subtracts the discharge emission. Depending on the set-up it may be necessary to apply corrections for self-absorption in the lamp and the possibility that the spectral bandwidth of the spectrometer is larger than both the emission line width from the lamp and the absorption line width.

The absorption of radiation in a plasma can be described by the LambertBeers law

$$
I_{v}(\ell)=I_{v}(0) \exp \left(-\kappa_{v} \ell\right)
$$

where

$I_{v}(0)$ and $I_{v}(l)$ are the specific intensities of the radiation entering and leaving the plasma $\ell$ is the length of the absorbing.path, and the absorption coefficient $\kappa_{v}$ is given by

$$
\kappa_{v}=\sum_{k} n_{k} \sigma k
$$

In equation (34) the summation is over all absorbing species and states, $n_{k}$ are the number densities and $\sigma_{v k}$ the absorption cross sections. If the incident radiation has a frequency $v=\left(E_{m}-E_{n}\right) / h$, then 1 ine absorption according to equation

$$
x_{n}+h_{v} \stackrel{\sigma_{n m}}{\rightarrow} x_{m}
$$

will be the dominant process if a strong transition $n \rightarrow m$ is chosen. Note that photoionization from the ground state is excluded in this case. For resonance lines and the strongest nonresonance transition $\Delta$, photoionization from some excited states is negligible and a measurement of $k(v) w i l l$ yield the population $n_{n}$ of the lower state. A discussion of detalled theory and experiment is given by Cabannes and Chapelle (1971). Examples of the diagnostic application of absorption spectroscopy to glow discharges are to be found in the papers by Greene (1978), Klinger and Greene (1981), Knights et al. (1982) and many others.

Use of absorption spectroscopy in visible and ir regions is frequently hindered by the plasma emission. Therefore, species effusing from the plasma, through a small orifice, have been trapped and isolated in a solid matrix of an inert gas at cryogenic temperatures ( 4 to $20 \mathrm{~K}$ ) and subsequently identified by absorption spectroscopy. This is the technique of matrix isolation spectroscopy described in the classical books of Bass and Broida (1960) and Minkoff (1960). Its application for elucidating the sputtering process has been described by Gruen et a 1. (1974). In the apparatus used by Veprek et al. (1975) for glow discharge deposition the molecular beam effuses from the 
discharge tube through a small orifice and is trapped on a liquid helium cooled, optically pollshed copper block simultaneously with a suitably chosen matrix gas such as $\mathrm{Ar}, \mathrm{Kr}, \mathrm{Xe}, \mathrm{N}_{2}$, etc. After the desired matrix has been deposited, the copper block, together with the reservoir for the liquified gases, is rotated through $180^{\circ}$ and the absorption spectrum of the matrix is scanned through a $\mathrm{KBr}$ window. A large number of papers demonstrate the applicability of this technique for detecting and measuring the concentrations of free radicals, atoms, as well as lons which are present in glow discharges without the problems resulting from plasma radiation and electron impact fragmentation, as in mass spectrometry.

Fluorescence spectroscopy. - A particle excited by the absorption of radiation may undergo radiative transitions in which light of the same or a different frequency is emitted. When such transitions occur between states of the same multiplicity the particle is said to emit fluorescence radiation, the intensity of which is proportional to the particle density. In principle the density of molecules, atoms and ions in the ground state as well as in a metastable or unstable excited state can be determined. Since fluorescence rise-times are considerably shorter than a microsecond, it is necessary to use laser pulse widths of the order of nanoseconds. Therefore, the technique has come to be known as laser-induced fluorescence (LIF). The importance of this technique as a plasma diagnostic tool was recently emphasized by Miller (1981) in a review articie.

With a continuous wave (CW) laser it is possible to continuously excite and detect fluorescence, but at a level much lower than the pulsed laser and often below the noise level resulting from the background emission. A tunable pulsed dye laser has a wider wavelength coverage (2500 to $8500 \AA)$, and the number of photons avallable during a pulse is considerably greater than that avaliable from a CW laser. By saturating a given transition with the pulsed laser, all the excited molecules can be made to emit in a time period comparable to the excited state 11fetime. This laser-induced signal may be considerabiy greater, over that given time period, than even relatively bright continuous background emission from the plasma. The high sensitivity of this technique was demonstrated by Bondybey and M1ller (1977). The technique also provides excellent spatial and temporal resolution.

Figure 21 is a schematic diagram of the set-up suggested by Milier and Bondybey (1980) for an LIF-experiment. The plasma reactor is equipped with long arms containing light baffles and Brewster-angle windows to minimize laser light scattering. The laser beam passes through the plasma along this axis and fluorescence is detected in a plane perpendicular to this axis by a photomultiplier. Often a diagnostic arrangement is configured with a pulsed dye laser and a gated detection system, as shown in figure 21 , to reject most of the continuous background emission from the reactor. An excitation spectrum is recorded by sweeping the laser frequency and monitoring the total fluorescence. In some experiments monochromator-based optical detection systems have been used to monitor the continuous emission from the reactor and/or to resolve the LIf wavelength resulting from a feature of the exc1tation spectrum obtained by tuning the laser.

Initially LIF was used mainly in the field of plasma wall interaction in magnetic confinement experiments and laser fusion research. A number of authors, among them Stern (1977), Burakov et al. (1977, 1978), Bogen and Hintz (1978), and Elbern et a1. (1977, 1978), demonstrated that besides neutral $H$ 
and $D$ atoms, several metals can be detected with high sensitivity. Elbern (1978) has reported measurements on the relaxation and diffusion of Fe-atoms in an argon glow discharge. Subsequentiy, Pellin et al. (1981) and Wright et a). (1981, 1982) used both CW and pulsed laser to measure the velocity distribution of sputtered $\mathrm{Zr}$-atoms

A number of species of the type encountered in plasma chemistry have also been observed by Cook et al. (1978), Katayama et a1. (1980), M117er and Bondybey (1977), Bondybey and Miller (1978, 1979), Miller et a1. (1979 a,b) and Sears et al. (1980). These include metastable states such as $\mathrm{N}_{2}\left(\mathrm{~A}^{3} \Sigma_{\mathrm{U}^{+}}\right)$ and $\mathrm{CO}\left(\mathrm{a}^{3} \pi\right)$, whose presence would be very difficult to infer from emission spectroscopy or mass spectrometry. Molecular ions $\left(\mathrm{N}_{2}^{+}, \mathrm{O}_{2}^{+}, \mathrm{CO}^{+}, \mathrm{CO}_{2}^{+}\right)$including moderately large organic cations such as $\mathrm{C}_{6} \mathrm{~F}_{6}{ }^{+}, \mathrm{C}_{6} \mathrm{H}_{3} \mathrm{~F}_{3}{ }^{+}$, and their analogues have also been detected, but, in general, the determination of absolute concentrations has been difficult. Free radicals such as $\mathrm{CH}, \mathrm{CH}_{2}, \mathrm{CN}$ and $\mathrm{OH}$ were observed in combustion experiments rather than glow discharge tubes. A review of this work is given by Eckbreth et al. $(1977,1979)$. With regard to glow discharge deposition the LIF technique has yet to be applied. of related interest is the work of Brueck and Pang (1983) who made spatially resolved measurements of $\mathrm{CF}_{2}$ concentrations and vibrational and rotational temperatures in $\mathrm{CF}_{4}, \mathrm{CF}_{4} / \mathrm{O}_{2}$ and $\mathrm{CF}_{4} / \mathrm{H}_{2}$ glow discharges of the type used for etching of $\mathrm{SI}$ and $\mathrm{SiO}_{2}$.

Recently, Walkup et a1. (1983) have combined LIF with laser optogalvanic (LOG) spectroscopy to study a glow discharge in $\mathrm{N}_{2}$. The LOG signals were measured by monitoring the voltage across the discharge through a dc blocking capacitor and processing this voltage with a boxcar average. The spatial distribution of $\mathrm{N}_{2}{ }^{+}$(which is closely related to the distribution of electric fields) was obtained by LIF and LOG methods. The measurements which included kinetic (translational) and internal (rotational and vibrational) energy distributions of $\mathrm{N}_{2}^{+}$suggested the importance of charge exchange collisions, particularly in the cathode sheath region. However, the kinetic energy of $2.2 \pm 0.7 \mathrm{eV}$ for $\mathrm{N}_{2}{ }^{+}$in a 1 torr $\mathrm{N}_{2}$ discharge measured by Doppler broadening is really too low. Nevertheless, with further developments LOG spectroscopy will provide a uniquely sensitive probe of positive fons in the substrate region of glow discharge deposition systems.

Coherent Ant1-Stokes Raman Spectroscopy (CARS). - Several systems have been observed to scatter radiations. The inelastic scattered radiations occur at lower (Stokes lines) or higher (anti-Stokes lines) frequencies than the frequency of the incident radiation, corresponding to the rotational or vibrational transition frequencies or both of the sample. This property is the basis of a technique which was initialiy demonstrated by Rado (1967) and has come to be known as coherent anti-Stokes Raman spectroscopy (CARS). With respect to glow discharges, and plasmas in general, CARS is better suited than fluorescence because of its excellent background light rejection capability. Besides the shorter wavelength-side spectral position of the signal the other advantages are the high signal power, signal coherency, and good spatial iresolution. For a review, see article by Nibler (1982).

A schematic diagram of a CARS set-up used by Hata et a1. (1983) is shown in figure 22. Typlcally, two laser beams of frequencles $v_{1}$ and $v_{2}$ $\left(v_{1}>v_{2}\right)$ are colinearly guided into a glow discharge to produce $2 v_{1}-v_{2}$ signal beam through nonlinear (third-order) susceptibility $x^{(3)}$ of the 
sample. The absolute value of $\left|x^{(3)}\right|$ is a function of $v_{1}$ and $v_{2}$, and resonantiy increases as $\left(v_{1}-v_{2}\right)$ approaches the Raman active transition frequency $v_{R}$ of the species under investigation in the discharge. Therefore, the CARS power signal, which is proportional to $|x(3)|^{2}$, can be treated as a function of $v_{2}$ when $v_{1}$ is fixed, and w117 exhibit some structure near the frequency $v_{2}=v_{1}-v_{R}$. Alternatively, $v_{1}$ may be mixed with a broad. $v_{2}$ source and a broad range of the CARS spectrum may be detected simultaneously by using an optical multichannel detector attached to a monochromator exit.

Figure 23 shows the CARS power signal as a function of $\left(v_{1}-v_{2}\right)$ for a $5 \mathrm{~mA}$ dc glow discharge in silane for (a) discharge off and (b) discharge on conditions. Here $C O$ is the reference signal at $2143 \mathrm{~cm}^{-1}$. The peak intensity of CARS signal is a measure of the number density of the species being investigated. Therefore,

$$
N^{2}=N Z\left[I\left(\mathrm{SiH}_{4}\right) / I(\mathrm{CO})\right] /\left[\mathrm{I}_{0}\left(\mathrm{SiH}_{4}\right) / \mathrm{I}_{\mathrm{O}}(\mathrm{CO})\right]
$$

where $N$ and I represent the number density and peak intensity of the CARS line, respectively, the suffix 0 denotes the discharge-off condition, 1.e., the plasma free gas flow. Since $N_{0}$ is known from pressure measurements, $N$ is readily evaluated.

Originally, CARS was applled by Nibler et a1. (1976) for the measurement of rotational and vibrational temperatures of a gas such as $D_{2}$ in a glow discharge. On the assumption of a Boltzmann equilibrium among only the lowest vibrational levels, Shaub et a1. (1977) made direct measurements of nonequilibrium vibrational levels populations of electrically excited $\mathrm{N}_{2}$ and found them to be much hotter than discharged $\mathrm{N}_{2}$. Since then some publications have appeared, among them we may cite Pealat et al. (1984) who showed that the population of vibrational states $v=0,1$, and 2 in a low pressure $\mathrm{H}_{2}$ discharge has a non-Boltzmann distribution and Hata et al. (1983) who measured the rotational temperatures and densities of species such as $\mathrm{H}_{2}$ and $\mathrm{SiH}_{4}$ in a silane glow discharge. The latter work is of particular interest since it demonstrated the existence of $\mathrm{SiH}_{2}$ radical $\left(2030 \mathrm{~cm}^{-1}\right)$ in its ground electronic state in concentrations of $\sim 7074 \mathrm{~cm}^{-3}$.

\section{Probe Analysis}

The electric probe method has found wide application in the classical physics of gas discharges. With proper care, the local plasma density $n$, electron temperature $\mathrm{KT}_{e}$, and plasma potential $\mathrm{V}_{\mathrm{p}}$ can be determined simultaneousiy by inserting a metallic electrode, usually a wire, into the plasma (fig. 24). The method has come to be known as the electrostatic (Langmuir) probe, first used by Langmuir and Mott-Smith (1924), and is based on the polarization of a plasma and the fact that plasmas do not obey Ohm's law. The current to the probe is measured as a function of the probe potential to obtain the volt-ampere characteristics, commoniy known as the probe characteristic (fig. 24).

Whereas the experimental procedure is simple, the theory has a tendency to become extremely complicated. Articles by Chen (1965). Schott (1968, 1971) and Cherrington (1982) and monographs by Swift and Schwar (1969) and Chung 
et a1. (1975) are excellent sources of detalled information for the interested reader. The only probe regime that can be reasonably understood is the region ( $p \leq 0.1$ tor $r$ ) where the mean free path $\lambda$ is very much greater than the probe size which in turn is very much greater than the Debye length, $\lambda_{D}$, the distance in a plasma over which significant deviations from charge neutrality, and therefore significant electrostatic fields, can exist.

It is easy to recognize three regions in figure 24 . Let us first consider region $I$, the ion saturation region. For the condition $T_{e} \gg T_{i}$ obtained in glow discharges the ions must enter the sheath with an energy $\mathrm{kT}_{\mathrm{e}}$. Since the ions are accelerated through a "pre-sheath" region to this energy value, the collected ion-saturation current $I_{1}$ becomes, as given by Chen (1965), approximately

$$
I_{1}=0.61 \mathrm{~A}_{p} n e\left(k T_{e} / m_{j}\right)^{1 / 2}
$$

where $A_{p}$ is the collection area of the probe. Thus if $\mathrm{T}_{e}$ is known we can evaluate the plasma density, $n$. Since lons are less affected than electrons by magnetic fields plasmas in such fields can be analyzed in this way. Only. When the sheath is thin compared to the probe radius, $r_{p} \gg \lambda_{D}$. the current is determined by the space charge-diode relationship, as discussed by Chen (1965), and the electron saturation current (region III) can be used to give a value for $n\left(k T_{e}\right)^{1 / 2}$.

The shape of the retarding fleld region (II) obviously is related to the distribution of electron energies, $f(E)$, and hence gives $k T_{e}$ when the distribution is Mawelilian:

$$
I_{e}=-(1 / 4) A_{p} n e\left(8 k T_{e} / \pi m_{e}\right)^{1 / 2} \exp \left(-e V_{\phi} / k T_{e}\right)
$$

where $v_{\phi}=v_{p}-v$ is the retarding potential. For $v_{p}>v_{f}(f i g .24)$, the ion current is small and a plot of $1 \mathrm{nI} e$ as a function of $v_{\phi}$ wili give $\mathrm{T}_{\mathrm{e}}$. For the non-Maxwellian condition of glow discharges the distribution is still isotropic; the electron current is

$$
I_{e}\left(V_{\phi}\right)=A_{p} n e\left(2 \pi e / m e^{2}\right) \int_{e V_{\phi}}^{\infty} E f(E)\left(1-e V_{\phi} / E\right) d E
$$

where $E=1 / 2 m v$ ? is the electron energy. Here $f(E)$ is normalized so that a Maxwellian distribution would be $f(E)=\left(m_{e} / 2 \pi k T_{e}\right)^{3 / 2} \exp \left(-E / k T_{e}\right)$. In this case, $f(E)$ can be obtained directly from the second derivative of the probe characteristic:

$$
f(E)=\left[m_{e}^{2} / A_{p} n e 2 \pi e^{3}\right]\left[d^{2} I_{e}\left(v_{\phi}\right) / d v_{\phi}^{2}\right] \mid E=e V_{\phi}
$$

'This is the widely used probe technique for determining the electron energy distribution function.

The plasma potential, $V_{p}$, can be determined by locating the function between regtons II and III ( $f$ ig. 24) or by measuring the floating potential $v_{f}$, at which the current nulls out, and calculating $v_{p}$. However, as 
pointed out by Koenig and Maissel (1970) and Coburn and Kay (1972), $V_{f}$ cannot be used to calculate $v_{p}$ in $r f$ discharges used for fllm deposition.

Vossen (1979) has shown that in these systems the measured potentials depend upon the relative area of the excitation electrode and of other grounded surfaces in contact with the discharge. Kay et al. (1980) have discussed these problems in some detail.

Double probes (fig. 25) are generally used for rf and microwave discharges where a reference electrode is usually not avallable (see Inspektor et a1. (1981)). Since both probes are insulated from the ground the probes float with the plasma and are unaffected by changes in $v_{p}$. As shown in figure 25 both probes will be at a negative potential with respect to the plasma since no net current can flow to the system. The circuit is balanced when the electron current flowing to one probe is balanced by the lon current flowing to the other. The currents are therefore 1 imited by the ion saturation currents for each probe. For this reason double probes are generally preferred when magnetic fields are present. As shown in figure 25 the $V$-I characteristic is found by overlapping the ion saturation characteristic of each probe. For the ideal situation where the electron saturation regions are flat, the current is given by

$$
I=I_{1} \tanh \left(e V / 2 k T_{e}\right)
$$

The electron temperature is determined by taking the slope of the curve at the origin (fig. 25):

$$
\left.\mathrm{dI} /\left.\mathrm{dV}\right|_{\mathrm{I}=0=\cdot(\mathrm{e} / \mathrm{kT}}\right) \mathrm{I}_{1(1)} \mathrm{I}_{1(2)} /\left[\mathrm{I}_{1(1)}+\mathrm{I}_{1(2)}\right]
$$

Once $k T_{e}$ is known, the plasma density can be computed from the ion saturation current. From figure 25 it is obvious that only a small fraction of the energy distribution of the electrons in the plasma, the high energy tall, is sampled by the symmetrical double probe arrangement. However, this difficulty has been overcome by the use of asymmetrical systems. Swift (1969) has shown that the complete distribution is sampled if the area ratio is sufficiently large. Groh and Reuschling (1981) have described an electronic analyzer for computing $T_{e}\left(10^{4}-10^{6} K\right)$ and $n_{e}\left(10^{11}-10^{13} \mathrm{~cm}^{-3}\right)$ directly from double probe characteristics of electrodeless glow discharges.

The foregoing discussion has neglected negative ions by assuming that

$$
n_{-} / n_{e} \ll\left(m_{-} T_{e} / m_{e} T_{-}\right)^{1 / 2}
$$

As Lergon et al. (1984) have shown glow discharges of halogen-bearing gases or oxygen have large concentrations of negative ions. In such cases the contribution due to negative ions must be included.

Probes have been used for diagnostics of sputter coating discharges and plasma deposition systems. Such applications are reviewed by Eser et al. (1978). Thornton (1978) and Clements (1978). Probe surface contamination affects probe characteristics and data interpretation. Clements (1978) has pointed out that the effects of contaminants on a probe surface can be minimized by blasing the probes with an $\mathrm{rf}$ voltage. 0liver et al. (1970) excited a double floating probe system with low-amplitude ( $\left.V_{b} \ll k T_{e} / e\right) r f$ whose frequency was between the plasma-ion and plasma-electron resonant frequencies and showed that surface oxide layers produced no effect on the 
measured values of $T_{e}$. and $n$ (even though dc measurements indicated changes of a factor of 2).

Nilinomi and Yanagihara (1979) used probes that were heated up to $1000 \mathrm{~K}$ with sheathed heaters inserted into them to prevent polymer films from depositing on the surface of the probe. Under film formation in an argon/benzene plasma the measured $f(E)$ showed considerable departure from a Maxwellian distribution. Yamaguchi et al. (1977, 1979) have described the effects of plasma-poiymerized styrene films on the V-I characteristics of single and double probes. Inspektor et al. (1981) have used floating double probes to characterize microwave plasmas in hydrocarbons under pyrocarbon deposition conditions and observed that $T_{e}$ and $n$ were not similar in their behavior with respect to external parameters such as gas pressure and composition, applied power and probe positions in the microwave plasmas. Mosburg, Jr. et al. (1983) have shown that, with proper care, electrostatic probes can be used for the measurement of $T_{e}$ and $n$ in $r f$ discharges in sllane, even though a film of semiconducting $S i$ is continuously deposited on the probe surface. In fact they suggest that the measurement may be helped by a continuous renewal of the surface. Probe analysis of other silicon containing glow discharges have been reported by a number of authors, among them Grossman et a1. (1982), Gleres (1983) and Avni et a1. (1983). Grossman et al. (1982) found that $T_{e}$ was independent of the $r f$ frequency, but $n$ was higher at a higher frequency indicating a frequency dependence of the degree of ionization. In dc discharges in which the probe was located in the negative glow Gieres (1983) found that the electron energy distribution cannot be described by a uniform temperature.

As Venugopalan (1983) pointed out, the use of electrostatic probes is fraught with dangers of not only contamination but also catalytic activity. Many of the probe materials such as Pt, $W, N 1$, etc. are well known catalysts for a number of reactions even under plasma conditions (Venugopalan and veprek, 1983). This is a field which needs to be investigated in some detail, particularly under deposition conditions.

\section{Mass Spectrometric Sampling}

Mass spectrometric sampling of a plasma is an intrusive technique in which the particle fluxes generally depend upon the sampling sheath thickness and voltage. Nevertheless, it has been applied to identify and measure particle concentrations and energies in glow discharges. In many instances mechanistic insight has been obtained for processes in the glow and for reactions (usualiy ion-molecule collisions) in a spatial or temporal afterglow.

For neutral species molecular beam methods developed by Foner and Hudson (1953, 1962) are still in vogue. The neutral beam is modulated between a sampling orffice located in a wall of the discharge tube and the conization chamber of the mass spectrometer and only the modulated component of the mass 'spectrometer output is recorded. This technique permits all neutral species, including radicals, to be detected with sufficient sensitivity. In the absence of modulation the sensitivity for detecting free radicals is lowered by several orders of magnitude. Recently. Hayashi et al. (1982, 1983) used photoionization mass spectrometry for observing $C_{m} F_{n}$ radicals produced in an rf plasma; the ionizer used noble gas resonance lines (Ar 11.6 and 11.8 $\mathrm{eV}, \mathrm{Kr} \mathrm{10.03}$, and $10.64 \mathrm{eV})$ or the Lyman $\alpha-1$ ine $(10.2 \mathrm{eV})$ of hydrogen. 
For lonic spectes a somewhat more complex experimental configuration is necessary. The lon beam which is extracted from the plasma through a sampling orifice must be focused into the mass spectrometer. If conventional magnetic sector instruments are used efther the plasma itself or the drift. tube of the mass spectrometer has to be operated substantially away from ground potentlal so as to achieve the large and variable kinetic energy of ions required by these instruments. Wagner and Brandt (1981) have described the use of such an instrument for studying the positive lon chemistry in Si/SF 6 system.

In recent years most glow discharge studies have been performed using the, quadrupole, ${ }^{9}$ first described by Paul, Reinhard and von Zahn (1958) and also known as a mass filter. During the last ten years or so there have been numerous pubilications on ion sampling from glow discharge deposition and polymerization systems using quadrupole mass spectrometers. We may refer here to papers by Kay et al. (1976), Smolinsky and Vasile (1977), Turban et al. (1979, 1982) and Carmi et al. (1982). Wagner and Veprek (1982, 1983) have described the application of mass filters for kinetic and chemical relaxation studies in Si/H system. Quadrupoles require only low energy ions (10 eV) and can be operated without serious problems if the ion beam has a significant energy spread. Their main disadvantage is low mass resolution. The ability to fonize and collect neutral species is usually incorporated in these mass spectrometers so that much neutral sampling work is done along with ion samp1ing. For example, Coburn and Kay (1971) and Purdes et al. (1977) describe the monitoring of fons and neutrais from plasmas through an aperture in the substrate table, behind which was mounted a differentially pumped quadrupole mass analyzer. Ion-molecule collistons in the sheath region and downstream from the sampling orifice affect the accuracy of ion densities measured this way. These effects have been discussed by a number of authors, among them Smith and Plumb (1973), Helm et a1. (1974), and Hasted (1975).

In glow discharges used for deposition the main concern is plasma-surface interaction rather than ion density. Ions formed in the sheath or downstream from the sampling orifice will have a lower energy than ions coming directly from the bulk plasma with an energy characteristic of the plasma potential, $v_{p}$. The collisional processes in the sheath region must be included to obtain the ion flux incident on surfaces. For determining the importance of collisional processes in the ion extraction Coburn (1970) and Coburn and Kay (1972) used an energy spectrometer between the sampling orifice and the mass spectrometer (see fig. 26). Komiya et al. (1977) have reported on the use of an ion energy analyzer in their mass spectrometric sampling of the incident ions on a substrate during deposition by hollow cathode discharge. Franklin et al. (1968), Vasile and Smolinsky (1973), Rowe (1975), and many others have used retarding potential techniques. Energy distribution obtained with defiection energy spectrometers are much more precise than those measured with retarding potentials. The latter, however, is compatible with line-of-sight sampling of the neutrals.

In their studies of ion-molecule reactions Boehme and Goodings (1966), 'Shahin (1969) and many others combined mass spectrometric sampling of positive ions with Langmuir-type probe diagnostics. Recently, Drevilion et al. (1980)

${ }^{9}$ The name originates from the fact that the device consists of four conducting cylinders. 
and Gieres (1983) applied such a combined diagnostics to characterize a dc glow discharge deposition system for silicon. For some years Lergon and Mueller (1977) have used wall probes for negative ion extraction in plasmas of strongly electron-attaching gases such as $\mathrm{SF}_{6}$. A typical wall probe consists of a piece of platinum melted into the Pyrex wall of a discharge tube and has an orifice size of $50 \mu \mathrm{m}$. Recently Lergon et al. (1984) have shown that the measurement of negative ion is a very sensitive method for the detection of deposited wall material.

\section{Magnetic Resonance Techniques}

Magnetic resonance methods depend on the gyromagnetic properties of the system to be diagnosed. When these properties are associated with electrons, electron paramagnetic resonance (EPR) is observed. Free atoms and free radicals are paramagnetic and the EPR technique has therefore been used for studying these species. Several reviews of gas-phase EPR have appeared, among them we may cite Westenberg (1969), Carrington et al. (1970), Brown (1972), and Miller (1981). The last one is addressed particularly to the problems encountered in plasma diagnostics. Since the detection sensitivity for free atoms and radicals is about $1011 \mathrm{~cm}^{-3}$ the technique is suitable for glow discharges.

In general, the absorption of microwave energy by free atoms and radicals placed in strong magnetic fields is studied. A free atom or free radical in a magnetic fleld has its energy levels split into their (otherwise degenerate) components which correspond to different orientations of the radical's magnetic moment along the magnetic field. This is, of course, well known as Zeeman splitting. In an EPR experiment the free atoms and free radicals must be contained in a microwave cavity (fig. $8(\mathrm{~b})$ ) and the applied magnetic field adjusted such. that the energy spacing between the Zeeman levels is equivalent to the resonant microwave frequency $(9 \mathrm{GHz})$ of the cavity. When this resonance occurs, photons are absorbed by the sample and the loss of power from the microwave field is detected. The energy hy of one of the otherwise degenerate Zeeman components $M_{J}$ is given by the equation

$$
h v=g_{j} \mu_{B}^{H M}
$$

where

H magnetic field

$v$ level's frequency relative to its zero value

g) "g-factor," which is determined by the species' magnetic moment

$\mu B$ Bohr magneton

$M_{j}$ projection of the angular momentum $J$ along the field direction.

Term symbols give all the necessary information for calculating $g_{j}$ (see Bethe and Salpeter (1957)); spectroscopic selection rule gives $\mathrm{MJ}_{\mathrm{J}}= \pm 1$ so - that the resonance field $\mathrm{H}_{r}$ is given by

$$
H_{r}=h v_{s} / g_{j} \mu_{B}
$$

where $v_{s}$ is the microwave frequency of the spectrometer. This simple theory ailows the prediction of absolute positions of atomic resonances usually in the absence of hyperfine structure. 
A number of atomic species such as $H\left({ }^{2} S_{1 / 2}\right), D\left({ }^{2} S_{1 / 2}\right), N\left({ }^{4} S_{3 / 2},{ }^{2} D_{5 / 2}\right)$, $0\left(3 p_{2}, 3 p_{1}\right), F\left(2 p_{3 / 2}, 2 p_{7} / 2\right)$ etc. have been detected in $g$ low discharges in the gas phase. Fluorine atom which is believed to play an important role in plasma etching process, however, has a nuclear spin and can exhibit hyperfine structure. In such a case previous studies of the species usually will suggest the line positions and shifts due to changes in $v_{s}$ can readily be estimated from equation (45). Among the free radicals detected we may mention $\mathrm{OH}, \mathrm{SH}, \mathrm{CF}, \mathrm{SF}, \mathrm{NO}, \mathrm{Cl0}, \mathrm{a} 11$ of which are in $2 \mathrm{\Pi}$. states, and $\mathrm{O}_{2}, \mathrm{SO}$, $\mathrm{SeO}$ in the $T_{\Delta}$ states. Since their $g_{j}$ values can be calculated (Carrington et al. (1970), M1ller (1981)), the resonance fields can be predicted.

Besides identification EPR spectra have also been used to obtain absolute concentrations of free radicals. The reader is referred to papers by Westenberg (1965) and Breckenridge and M1ller (1972) for detalls and precautions. To clte a case in support, consider $0_{2}$ which has transitions that are magnetic dipole in nature and exhibits a five-line spectrum, four of which correspond to ${ }^{1} \Delta_{g}$ state and the fifth (strongest) to the ground state $X^{3} \Sigma_{\bar{g}}$. The integrated EPR signals of a given species are linearly proportional to the concentration of that species. Since ground-state $\mathrm{O}_{2}$ is a stable gas its concentration can be measured, say by a pressure measurement. Thus, by measuring the intensity of $\mathrm{O}_{2}\left(x^{3} \Sigma_{\overline{\mathrm{g}}}\right)$ in a given spectrometer, the strength of the lines of any other atomic or molecular spectes will give directly its absolute concentration. For heteronuclear species which have electric dipole transitions $N O$ is used in place of $0_{2}$ as a calibrant gas.

Whereas the possibility exists for measurements of the absolute concentrations of free radicals in gas phase, there have been no widespread application of EPR technique for in situ experiments in glow discharge deposition systems. In a recent investigation Avni et a1. (1983) used an indirect method in which the radicals in different locations of pyrocarbon and silicon plasmas were adsorbed and stabilized on preheated alumina and then transferred to an EPR tube for measurement. Since the radicals are adsorbed on solid, the resolution of the spectrum is poor and radicals cannot be identified (fig. 27). In the condensed phase, the magnetic moment of the species is determined usually by an unpaired spin (S) so that species with $S=0$ are not detected by EPR. 10 During the transfer the material invariably comes in contact with alr and molsture. Morita et a1. (1971, 1976). Tkatschuk et al. (1977) and Scott et al. (1979) have shown that exposure of flims to air causes a rapid decrease in the intensity of the original signal and even the appearance of new signals. This has been reconfirmed by Grenda and Venugopalan (1980) in an in situ experiment which also demonstrated the dependence of the signal on the film structure.

10 In the gas phase, however, there is the possibility of unquenched electronic angular momentum (L) which can give rise to a magnetic moment independent of the spin. The combination of orbital magnetism and electron spin can yield better Zeeman effects than typically encountered in condensed phases. 
Nevertheless, the signal intensity serves as an indicator for the radical concentration in the discharge. As Scott, et a1. (1978) pointed out the line shape is usually neither Lorentzian nor Gaussian and the relative area (A) under the absorption curve must be approximated by the product of the peak-topeak amplitude and the square of the peak-to-peak linewidth. The number $N_{p}$ of spins in the $f 11 \mathrm{~m}$ is calculated from the $N_{s}$ of spin in a standard (such as DPPH) using the equation

$$
N_{p}=N_{S}\left(A_{p} G_{s} M_{s} / A_{S} G_{p} M_{p}\right)
$$

where

G amplifier gain

M modulation amplitude (In Gauss or Tesla).

It must be pointed out that the presence of unpaired spins in films is due not only to the incorporation of gaseous free radicals formed in the discharge but also to the formation of radicals from the polymer materlal through the process of bond rupture caused by uv radiation in the plasma (section IID). This was demonstrated by Yasuda (1976) and Morosoff et al. (1976) by using ESR probes made of glass rods and polyethlene-coated tubes.

The application of EPR method to the study of trapped radicals in frozen matrices is complicated because of magnetic anistropies, matrix shifts, power saturation, etc., which result in severe line broadening, line distortion, and unusual intensity distributions. Despite these complexities the published literature for the past three-decades is rich in EPR work on condensates from glow discharges.

\section{REVIEW OF RESULTS}

In theory it is concelvable that almost any substance can be decomposed to a certain degree in a suitable glow discharge. In fact several hundreds of substances have been subjected to glow discharges. While a review of the results from the standpoint of starting materials is desirable for mechanistic purposes, interest in glow discharge deposition has evolved because of the mechanical and electrical properties of the films obtained. For this reason in this review we shall consider the deposition of three categories of films: metallic, semiconducting, and insulating.

\section{Metali1c Films}

The deposition of metalilic films in or near the plasma region was reported by McTaggart $(1965,1967,1969)$ who decomposed the vapors of the alkali and alkaline earth metal halides in the presence of a noble gas or $\mathrm{H}_{2}$ in a microwave discharge operated by a waveguide resonator. Although the observed idissociation rates could not be correlated with bond energies in the starting materials, the dissociation of alkali metal haltdes (MX) appeared to proceed as

$$
M X+e^{-}=M+X^{-}
$$

The alkaline earth metal halides $\left(M X_{2}\right)$, on the other hand, yielded deposits which consisted of equimolar mixtures of the metals and dihalides thus: 


$$
\begin{gathered}
M X_{2}+e^{-}=M X+X^{-} \\
2 M X=M+M X_{2}
\end{gathered}
$$

Glow discharge sputter deposition is preferable for producing relatively pure metallic films. This widely employed technique has been reviewed by a number of authors, and recently by Vossen and Cuomo (1978) from a process viewpolnt. As early as 1852, Grove used this method of metallic film deposition, whereby a dc glow discharge is established between a plate of source material (cathode) and the substrate (anode) in a chemically inert gas. The early literature on the subject is well documented by Glockler and Lind (1939). Basically, removal of the cathode materlal results from the bombardment of the cathode by energetic positive ions of the discharge. The process is referred to as "cathodic sputtering" and is the result of energy and momentum transfer from the bombarding lons.

A binary collision is characterized by the transfer function $4 \mathrm{~m}_{j} \mathrm{~m}_{\mathrm{t}} /$ $\left(m_{1}+m_{t}\right)^{2}$, where $m_{1}$ and $m_{t}$ are the masses of the incident ion and target species, respectively. Sputtered atoms come from the surface layers of the target so that we would expect the sputtering yield $S$ to be proportional to the energy deposited in a thin layer near the surface, NL in figure 13. sigmund (1969) has given the following expression for the sputtering yield, S:

$$
S=\left(3 \alpha / 4 \pi^{2}\right)\left[4 m_{j} m_{t} /\left(m_{j}+m_{t}\right)^{2}\right] E / U_{0}
$$

where

$E$ energy of the incident ion

$U_{0}$ surface binding energy of the material being sputtered

$\alpha$ monotonic increasing function of $m_{t} / m_{1}$ which has values of 0.17

for $m_{t} / m_{j}=0.1$, increasing up to 1.4 for $m_{t} / m_{j}=10$.

In practice the sputtering yield rises rapidly from an apparent threshold which is approximately equal to the heat of sublimation and is usually below $100 \mathrm{eV}$. Above $100 \mathrm{eV}$ it becomes a slowly varying function of $E$ until it reaches a broad maximum at energies which are higher than would normally be encountered in a glow discharge. Selected data are given in table I for $\mathrm{Ar}^{+}$ fons at various energies. Vossen and Cuomo (1978) have collated data for a number of ions. The interested reader is referred to articles by winters $(1976,1980)$ for further detal1s.

So far we have considered cathodic sputtering by positive ions. In glow. discharge systems, bombarding ions are by no means monoenergetic. Sputtering yields given in table I are therefore useful only to give a rough indication of the deposition rate of sputtered films. Further, the flux of neutrals is about $10^{4}$ times greater than the arrival rate of sputtered material. Besides ground-state neutrals a further source of bombardment is due to excited neutrals, of which metastables of the sputtering gas would be most abundant -(fig. 13). A major source of charged particle bombardment at the anode is due to electrons the energy spectrum of which was measured by Ball (1972) and Chapman et a1. (1974). They found that the fast electrons emitted from the target by ion and other impact can have a major influence on the structure and properties of the growing $\mathrm{film}$ on the substrate (anode). Negative ions sputtered from the target are accelerated across the discharge and collide 
with the anode. As Hanak and Pellicane (1976) and Cuomo et al. (1978) showed the sputtering produced by these negative lons not only reduced the amount of target materlal deposited on the anode, but, in some instances, produced steady-state sputter removal of the anode materlal itself. Finally, photons produced during ion or electron bombardment on any surface and from relaxation of excited atoms in the glow can affect the growth of a film.

If the substrate is different from the anode, a blas potential (usualiy negative) is applied to the substrate holder, so that the growing film is subject only to positive ion bombardment. This is known variously as "bias sputtering" or "ion plating." 11 Coburn (1970) identified the mass and energy of ions bombarding the substrate, with and without bias, in a dc discharge in argon with Cu cathode. Although the mass spectra from rf discharges were very simflar, there is a considerable difference in the ion energy distribution from the two types of discharge. Data such as these have to be carefully interpreted, as the ion current magnitude cannot simply be equated with ion abundance; the quadrupole spectrum tends to underestimate higher mass lons, and its sensitivity also depends on ion energy. Sputtered particles that were ejected from the cathode as positive ions could not reach the sampling aperture because of the large retarding field for positive ions in the cathode fall region of the discharge. Therefore, the observed sputtered material was ejected from the cathode as neutral atoms and subsequentiy ionized in the discharge. A similar observation was made in the 1920's by V. Hippel (1926) and Baum (1927) from their spectroscopic work. Coburn (1970) measured the ${ }^{63} \mathrm{Cu}^{+}$ion current as a function of the cathode position with and without bias voltage $(f 1 g .28)$, the former representing the deposition profile on the anode plane.

In an rf diode sputtering system Coburn and Kay (1972) found that the energy distribution of low mass tons were broadened significantiy by rf modulation during their passage through the plasma - substrate sheath. Collisional processes in the sheath region were found to influence the energy distribution of singly charged ions in the gas phase, but to a much smaller extent than in the target sheath. By using essentially the same mass/energy analysis technique Komiya et ai. (1974) found the relative intensity ratio of $\mathrm{Cr}^{+}$: $\mathrm{Ar}^{+}: \mathrm{Cr}^{++}$as 80:16:4 in a hollow cathode discharge used for $\mathrm{Cr}$ deposition. The energy distribution of $\mathrm{Cr}^{+}$which they measured ( $\mathrm{flg} .29$ ) indicated that $\mathrm{Cr}^{+}$is produced in a region that is at a potential of several to ten volts higher than that of the vacuum wall. More recently, Ziemann and Kay (1982) and Zlemann et al. (1983) have shown that the process of f1lm deposition by bias sputtering in a low pressure (1 mtorr) supported dc discharge can also be analyzed in terms of the energy delivered to the growing $f 11 \mathrm{~m}$ by the bombarding gas tons per arriving flim atom.

17 Initialiy, the term "Ion plating" was used by Mattox (1964) in reference to a process in which the deposition source was a thermal evaporation if llament instead of a sputtering target and the substrates were connected to a dc sputtering target. Lately, it was applied by Mattox (1973) to any process in which the substrate is subjected to purposeful ion bombardment during film growth in a glow discharge environment. 
Greene and Sequeda-0sorfo (1973) have correlated sputtering parameters and results of optical emission intensity measurements for Cu $(324.75 \mathrm{~nm})$ in an Ar discharge. They also give a brief review of earlier work on spectroscopic diagnostics of glow discharge sputtering. As shown in figure 30 the "total" intensity which is the area under the I versus $t$ curve is proportional to both the total change in target mass and the total thickness of deposited film. Since $I \times t$ is proportional to the film thickness the sticking coefficient must have remained constant within the sputtering parameters studied ( $p=90$ mtorr, $V=2$ to $3.5 \mathrm{kV}$ ). However, for very high sputtering rates or for heated substrates, we would expect a deviation from linearity as the sticking coefficient decreases. That the emission intensity is proportional to the sputtering rate implies that the probability of target atom excitation in the discharge was constant under their experimental conditions. A possible explanation is that the maximum concentration of sputtered atoms occurs in the cathode fall region where the electron energy is high and the relative probabllity of electron collision excitation is small, that is, the high energy tall of the cross section for electron impact excitation of neutral atoms. The results for In shown in figure 31 are, however, typlcal of optical emission data obtained by varying the Ar pressure at constant target voltage. The divergence between the cathode fall peak and the negative glow peak appears to indicate an increasing loss of sputtered material due to gas phase scattering of sputtered atoms in transit from the target to the substrate.

Greene (1978) has determined the spatial distribution of sputtered Cu atoms by absorption spectroscopy and showed that the deposition rate at the substrate for a parallel-plate reactor is proportional to $1 / p(d N / d x)$ where $\mathrm{dN} / \mathrm{dx}$ is the anode side slope of the sputtered atom distribution curve.

Greene (1978) has also investigated the effect of negative substrate bias $V_{b}$ on the sputtering rate of $C u$ as a function of its number density in the discharge. Figure 23 shows that $N_{\text {max }}$ varied 1 inearly with the target sputtering rate for $v_{b}<100 \mathrm{~V}$ : for $v_{b}>100 \mathrm{~V}$. Nmax increased at a faster rate due to resputtering from the film contributing a measurable fraction of the Cu atoms to the discharge, as well as to a siight increase in the residence time of sputtered atoms in the discharge. The resputtering rate $R_{r}$ was calculated by using the equation

$$
R_{r}=R_{S}(1-r)[1-\xi]=R_{S}(1-r)-R_{a}
$$

Where the accumulation rate $R_{a}=R_{s}(1-r) \xi, r_{s}$ is the sputtering rate, $r$ is the fraction of material that is lost from discharge by scattering and $\xi$ is the probability that the impinging atom will be incorporated into the film. The last equality is obtained for the case that the thermal sticking probability is unity. The inset in figure 32 shows that the resputtering rate during deposition is linearly proportional to the excess signal, $\Delta N$, for various values of $V_{b}$ :

- Most of our discussion until now has been concerned with the sputtering of a single metal. The sputter deposition of alloys, and multicomponent films in general, is complicated because the components may have different sputtering yields, condensation coefficients and transport properties. An example of this is found in Coburn's (1970) work using an aluminum-bronze (Fe, 3 at.\%; A1, 15 at. \%; Cu 82 at. $\%$ ) cathode in a dc discharge in argon. The positiveion mass spectrum showed a relatively large abundance of $\mathrm{Fe}^{+}$which could 
not be explained. Greene et al. $(1975,1978)$ used emission spectroscopy to detect not only the discharge gas but also the elements from alloy targets such as Mone1 K-500 (N1, 65.33 wt. $\%$; Cu, 29.31 wt. $\%$; Fe, 1.01 wt. 8 ) and Inconel 718 ( $N 1,53.25$ wt. $\$$; Fe, 18.14 wt. $\%$; $\mathrm{Cr}, 18.01$ wt. 8 ). Upon initlal bombardment of the surface the constituents with the highest sputter yield (table I) is preferentially removed from the surface, enriching the surface layer in the lower sputter yield material (segregation) until a steady state is reached. The mean free paths of the sputtered material should not be very different, but the condensation coefficient of a multicomponent target will be different, causing an effective change in the composition of the depositing alloy. Resputtering will, of course, deplete the film of the component with the higher sputtering yield, and will be particularly effective under bias conditions.

"Chemical sputtering" may occur if reactive gases are present in the deposition system. It involves the reaction of an excited neutral or ionized gas with a surface to form volatile compounds. The technique is mainiy used for plasma treatment of organic surfaces and for etching in plasmas. As early as 1926, Guentherschulze observed that $\mathrm{C}, \mathrm{Se}, \mathrm{As}, \mathrm{Sb}$, and Bi formed volatile hydrides in a cold-cathode discharge in hydrogen and that these compounds dissociated outside of the cathode dark space to deposit metallic films. However, a relatively recent study of the chemical sputtering of graphite in an oxygen plasma by Holland and 0jha (1976) indicated that impacting ions sputtered C-0 compounds without their dissociating in the plasma. Also, Hanak and Pellicane (1976) found that etching of glass substrates rather than film deposition occurred in systems with reactive anion targets of $\mathrm{TbF}_{3}$ and $\mathrm{TbCl}_{3}$. Such glow discharge systems have not been analyzed in any detall.

Neutral metal dimers have been observed during the sputtering of metal targets. For early work in this fleld, the reader is referred to papers by Honig (1958), Woodyard and Cooper (1964), and Dechsner and Gerhard (1972). Coburn et a7. (1974) made a mass spectrometric study of the sputtered species arriving at the substrate in an $r f$ diode sputtering system when varlous metal oxide $\left(M_{x} O_{y}\right)$ targets were used. Figure 33 shows the extent to which species $M 0^{F}$ are observed relative to the species $M^{+}$as a function of $M-0$ bond energy. If the species $M O$ were ionized in the discharge with an efficiency comparable to that for the ionization of the species $M$, then molecular sputtered species MO constitute a sizeable fraction of the total sputtered material particularly if the $M-0$ bond energy is large.

\section{Semiconducting Films}

The glow discharge deposition of semiconductor fllms, particulariy silicon, has been extensively studied. Since the early experiments of Schwarz and Heinrich (1935) numerous investigators have decomposed sllane $\left(\mathrm{SiH}_{4}\right)$ under a variety of conditions in an effort to prepare pure Si. However, the deposited film is largely hydrogenated amorphous silicon, sometimes also -called "polysilane," a-Si:H, or simply a-Si. The preparation of polycrystalline S1, pc-S1, and Ge fllms was accomplished by Veprek and Marecek (1968) who used the chemical transport of $\mathrm{Si}$ and $\mathrm{Ge}$ in a glow discharge in $\mathrm{H}_{2}$. More recently, microcrystalline silicon, $\mu \mathrm{C}-\mathrm{Si}$, has been deposited by the decomposition of $\mathrm{SiCl}_{4}$ and $\mathrm{SiHCl}_{3}$ and their mixtures with $\mathrm{H}_{2}$ in glow discharges. During the past decade there have been numerous publications on the analysis for characterization of these flims. There have also been several experiments 
to deplete their $\mathrm{H}$ or $\mathrm{Cl-content.} \mathrm{In} \mathrm{view} \mathrm{of} \mathrm{their} \mathrm{commercial} \mathrm{importance} \mathrm{the}$ preparation of doped or ion-implanted silicon fllms has also been a significant field of activity. LeComber and Spear (1979) and Bauer and Bilger (1981) have discussed some of the discharge conditions which influence the properties of the films. It is only during the last five years or so, serious efforts have been made to study the basic processes which result in different types of. silicon.

Glow discharges in silicon hydrides. - Mosburg, Jr. et al. (1983) have reported probe measurements of electron temperature and density in $\mathrm{rf}$ discharges in silane (fig. 34) and B-doped silane, even though a film of Si was continuousiy deposited on the probe surface. Their work suggested that the measurements may be helped by a continuous renewal of surface. During typical depositions of thin films of a-Si, values of $k T_{e}=2$ to $2.5 \mathrm{eV}$ and $n_{e}=$ (1 - 1.5) $\times 10^{9} \mathrm{~cm}^{-3}$ were obtained in stlane plasmas at $72 \mathrm{mtor}$ and $1.2 \mathrm{~W} \mathrm{rf}$ power. By using microwave interferometry 12 Turban et al. (1979) measured the mean electron density, $\bar{n}_{e}$, of an $r f$ discharge in 5 percent $\mathrm{SiH}_{4}-\mathrm{He}$ as a function of the $r f$ power (f1g. 35). De Rosney et al. (1983) deduced electron temperatures ranging from 1.6 to $2.5 \mathrm{eV}$ from the ratio of the $\mathrm{Si}$ $(288.2 \mathrm{~nm})$ to $H(656.3 \mathrm{~nm})$ emission intensities and the cross sections. 1isted by Perrin and Schmitt (1982). All these values appear to be reasonable for the given set of experimental parameters such as gas pressure and discharge power/frequency.

Hata et al. (1983 a,b,c) have used the CARS-technique for spatially resolved density measurements of $\mathrm{SiH}_{4}$ molecules and their rotational temperatures. In addition, they have detected for the first time the elusive $\mathrm{SiH}_{2}$ in a hydrogen-diluted silane $\left(\mathrm{SiH}_{4} / \mathrm{H}_{2}=1 / 9\right)$ plasma. Interestingly enough, 0 'Keefe and Lampe (1983) have also detected $S_{1} H_{2}^{\star}$, but by the OES technique in $\mathrm{SiH}_{4}$ and $\mathrm{SiH}_{4}-\mathrm{SiF}_{4}$ mixtures irradiated by a pulsed $\mathrm{CO}_{2}$ laser. Since $\mathrm{SiH}_{2}$ is considered to be the most probable species dominating a-S1 deposition (Kampas and Griffith, 1981), further application of the CARS technique is to be expected to elucidate the role of $\mathrm{SiH}_{2}$ in the surface reaction. For the present, optical emission spectroscopy (OES) and mass spectrometry (MS) are the two techniques widely used for species identification and concentration measurement in these systems. In the following sections we shall briefly survey recent papers on these two topics.

\section{Optical Emission Spectroscopy}

Griffith et al. (1980) and Kampas and Griffith (1980) Identified optical emisston from the species $\mathrm{H}, \mathrm{H}_{2}, \mathrm{Si}$, SiH and several impurity species in silane discharges. Perrin and Delafosse (1980) found that the rotational levels of the $A^{2} \Delta$ state of $\mathrm{SiH}(4127 \AA)$ are abnormally excited. Further

${ }^{12}$ The phase shift introduced by a plasma column of radius $R$ gives the mean electron density $\left\langle n_{e}\right\rangle$ according to

$$
\Delta \phi=4 \pi(R / \lambda)\left[\left(1-<n_{e}>/ n_{C}\right)^{1 / 2}-1\right]
$$

where $\lambda$ is the mean free space wavelength and $n_{c}$ the cut-off electron density. 
work by Kampas. and Griffith (1981) and Kampas (1983) using collision partners such as $\mathrm{N}_{2}$ and $\mathrm{Ar}$ have revealed that $\mathrm{H}$ and the emitting excited states of $\mathrm{Si}$ and SiH are primary products of the silane decomposition. They also found that the electron concentration decreased and the average electron energy increased as $\left[\mathrm{SiH}_{4}\right]$ was increased in $\mathrm{SiH}_{4}-\mathrm{Ar}$ mixtures. This dependence explains the work of Knights et a1. (1981), which showed that the deposition rate of a-Si from silane - noble gas mixtures is not proportional to $\left[\mathrm{SiH}_{4}\right]$.

In their later work Knights et a1. (1982) measured the vibration-rotation bands of the electronic ground state of $\mathrm{SIH}$ and deduced vibrational and rotational temperatures of this species to be 2000 and $484 \mathrm{~K}$, respectively. This suggested that for all dissociative states of $\mathrm{SiH}_{4}$ produced by electron impact leading to $\mathrm{SiH}$ formation there is a large excess of energy released in excitation of the fragments. Knights et al. (1982) also measured the infrared absorption spectrum of $\mathrm{SiH}_{4}\left(v_{3}\right.$ band at $5 \mu$ ) and used it to identify the $\mathrm{SiH}_{4}$ absorption from the plasma. The rotational and vibrational temperature of 300 and $850 \mathrm{~K}$ thus deduced for $\mathrm{SiH}_{4}$ is consistent with a nonequilibrium population driven by direct electron impact excitation of "rovibration" and subsequent collisional quenching.

A typical OES spectrum obtained by Matsuda et al. (1980) and Matsuda and Tanaka (1982) from a pure $\mathrm{SiH}_{4}$ glow discharge plasma ts shown in figure 36. They found that the OES intensity ratio of $\mathrm{H}^{*}$ to $\mathrm{SiH}^{*}$ is strongly correlated with the median wave number $\widetilde{v}_{m}$ of the Si-H stretching absorption band of the deposited film over a wide parameter space of the plasma conditions including inert gas ( $\mathrm{He}, \mathrm{Ne}, \mathrm{Ar}$ ) dilution of $\mathrm{SiH}_{4}$. The paper of Matsuda and Tanaka (1982) also describes the phenomenological relationship between the OES data and the properties of the deposited film.

In their later work Matsuda et al. (1983) studied $\mathrm{Si}_{2} \mathrm{H}_{6}$ discharges as we11. Figure 37 shows the deposition rate of a-Si films from $\mathrm{SiH}_{4}$ and $\mathrm{St}_{2} \mathrm{H}_{6}$ glow discharges in dlode and triode reactors plotted against the line intensity of $\mathrm{SiH}$ observed by OES during deposition. As is evident, the deposition rate of a-Si from the $\mathrm{SiH}_{4}$ glow discharge is proportional to the line intensity of $\mathrm{SiH}$ emission $(4127 \AA)$. No such simple behavior was found for $\mathrm{Si}_{2} \mathrm{H}_{6}$ discharges. From the quantitative measurement of the luminosity of the SiH emission, Matsuda et al. (1983) estimated the density of $\mathrm{SiH}^{\star}$ to be $3 \times 10^{6} \mathrm{~cm}^{-3}$. Since this value is 3 to 4 orders of magnitude lower than the density of species required for explaining the observed deposition rate, it was concluded that $\mathrm{SiH}^{*}$ is not a direct precursor for a-Si deposition but other species correlated with $\mathrm{SiH}^{*}$ (possibly SiH neutral radicals) are responsible for the deposition from $\mathrm{SiH}_{4}$ plasma. By measuring the deposition rate as a function of the separation between the mesh and the substrate electrode in a triode reactor Matsuda et al. (1983) determined that the SiH radical in $\mathrm{Si}_{2} \mathrm{H}_{6}$ plasma has a much shorter 11 fetime than that in $\mathrm{SiH}_{4}$ plasma.

Optical emission spectroscopy has also been applied by Marcyk and Street'man (1977) for measuring As-implanted S1, and by Zesch et al. (1980), among others, for measuring B-dopant concentrations in a-Si. 


\section{Mass Spectrometry}

Haller (1980) observed high relative abundances of ions containing more than one Si atom, $\mathrm{Si}_{j} \mathrm{H}_{k}{ }^{+}$, and suggested that their most rapid reactions in a silane plasma are charge transfer and formation of new Si-Si bonds upon collision with $\mathrm{SiH}_{4}$. Drevilion et al. (1980) found that $\mathrm{SiH}_{3}{ }^{+}$was the dominant ion by mass spectrometric sampling of a multipole discharge in 80 percent $\mathrm{SiH}_{4}-20$ percent $\mathrm{H}_{2}$.

Based on a mass spectrometric analysis of the positive ions and neutrals in $\mathrm{SiH}_{4}-\mathrm{He}, \mathrm{SiH}_{4}-\mathrm{D}_{2}$, and $\mathrm{SiO}_{4}$ - He plasma Turban et al. (1982) proposed the scheme shown in figure 38 for the deposition of a-Si flims. They observed ion - molecule, disproportionation, insertion and abstraction reactions involving the following active species: $\mathrm{SiH}_{2}, \mathrm{SiH}_{3}, \mathrm{H}, \mathrm{SiH}_{2}{ }^{+}$, and $\mathrm{SiH}_{4}$. Their study of the species flux to the walls of the discharge tube showed that free radicals $\mathrm{SiH}_{n}(n=0$ to 3 ) are the gaseous precursors of the f $11 \mathrm{~m}$ and that heterogeneous reactions of these radicals, H atoms, and ions at the walls control the composition and the structure of the films. The possibility that $\mathrm{SiH}_{2}$ and/or $\mathrm{SiH}_{3}$ radicals play an important role in the fllm formation was also suggested by Knights (1980) and Scott et al. (1980). Apparently the S1-H bonds in the film are produced partiy from $S_{1 H}$ radicals and partly from the direct incorporation of $H$ atoms into the growing film. The $H$-atom etching of a-Si films, which perhaps initiates the Si chemical transport observed by Veprek and Marecek (1968), indicates some reversiblitity in the deposition process (fig. 38).

Matsuda and Tanaka (1982) combined OES with MS for characterizing the glow discharges of $\mathrm{SiH}_{4}, \mathrm{Si}_{2} \mathrm{H}_{6}$, and $\mathrm{SiH}_{4}$-inert gas mixtures under conditions of a-Si deposition. They found that the density of ionic species, dominantiy $\mathrm{S}_{1} \mathrm{H}_{3}{ }^{+}$, is lower than that of neutral radicals by 4 to 5 orders of magnitude. Their blased substrate experiments showed that ionic species gave no discernible change in the deposition rate but strongly affected the quantity of hydrogen introduced into the $\mathrm{fllm}$ in the form of $\mathrm{H}^{+}$. The deposition rate could be well correlated with the MS data and analyzed quantitatively in terms of the $H$ to $S i$ ratio in the plasma. On the basis of mass spectrometric measurement, Matsuda et al. (1983) have also demonstrated that the crystallite size is strongly affected by the amount of lonic species impinging on the growing surface of $\mathrm{c}-\mathrm{Si}$.

Chemical transport in hydrogen plasma. - Chemical transport in a low pressure plasma of hydrogen has been used for preparing films of several materials since the formation of $\mathrm{pC}-\mathrm{Si}$ and Ge films was reported by veprek and Marecek (1968). The transport takes place only th the direction of increasing gas temperature, but the discharge current density is nearly constant between the charge and the deposition zone. Through a series of refinements in technique Veprek et a1. (1981) have found it possible to deposit polycrystalline films of silicon at temperatures as low as $80^{\circ} \mathrm{C}$. For a long time the emphasis in these investigations have been on the properties of the films and their control rather than understanding their formation via analysis of the glow discharges (see Veprek, 1980, 1982).

Recently, Wagner and Veprek (1982) made a mass spectrometric study of a deuterium discharge over c-Si powder, a silane discharge, and a deuterium discharge over a-Si deposited by a silane discharge. They found that at long residence times $\left(>5 \mathrm{~s}\right.$ ) the steady-state signal heights of $\mathrm{SiH}_{2}^{+}$(silane 
discharge) and $\mathrm{SID}_{2}{ }^{+}\left(S i / \mathrm{O}_{2}\right.$ discharge) are the same even though the two systems approach steady-state from opposite sides. The equivalence of the signal heights at long residence times means that the rates of production. of silane (forward reaction)

$$
\mathrm{Si}(\mathrm{s})+(\mathrm{x} / \mathrm{m}) \mathrm{H}_{\mathrm{m}}(\mathrm{g}) \rightleftharpoons \mathrm{SiH}_{\mathrm{x}}(\mathrm{g})
$$

and the removal of silane (reverse reaction) are equal. For this to be the case the authors proposed that a "partial chemical equilibrium" (nonisothermal conditions!) must have been reached in the $\mathrm{Si} / \mathrm{H}_{2}$ discharge. The kinetic parameter controlling the extent of departure of the system from such a state is the ratio $\tau / t_{\text {res, }}$, where $\tau$ is the characteristic time of reaction (eq. (52)), either from the educt or product side, and $t_{\text {res }}$ is the mean residence time of the species in the reaction zone. This work is significant in that the authors were able to delineate the conditions under which a-Si $\left(t_{\text {res }} / \tau<1\right)$ and $c-S i\left(t_{\text {res }} / \tau \geq 10\right)$ fllms are deposited.

In their later work, Wagner and Veprek (1983) applied a chemical relaxation technique to show that both $\mathrm{SiH}_{4}$ and $\mathrm{H}_{2} / \mathrm{Si}(\mathrm{s})$ discharges operating under the same conditions of pressure, discharge current density, temperature, and residence time display the same kinetic response as well as sllane concentration when they are at "partial chemical equilibrium", 1.e., $t_{\text {res }} \gg$ $\tau$. Since the formation of $\mathrm{H}_{2}$ and the decomposition of sllane occurred on the same time scale, a single step mechanism of silane decomposition was proposed:

$$
\text { - } \mathrm{SiH}_{4}=\mathrm{SiH}_{2}+\mathrm{H}_{2}
$$

Glow discharges in chlorosilanes. - The deposition of c-Si from glow discharges in $\mathrm{SiCl}_{4}$ and $\mathrm{SiHCl}_{3}$ and their mixtures with $\mathrm{H}_{2}$ and/or noble gases has been studied. Bruno et a1. $(1979,1980,1981)$, Katz et a1. (1980), Gafri et al. (1980), Grossman et a1. (1981, 1982a) and Grimberg et a 1. (1982) have described the dependence of the deposition rate on parameters such as position of the substrate in the reactor, gas pressure and composition, discharge power and frequency, etc. Grossman et al. (1982b) also characterized an rf plasma in $\mathrm{Ar}+10$ vol $\% \mathrm{H}_{2}$ using a floating double probe; based on this characterization they interpreted the deposition results from an $\mathrm{rf}$ plasma in a gas mixture containing 2.5 vol $\% \mathrm{SiCl}_{4}$ and 7.5 vol $\$ \mathrm{H}_{2}$ in Ar. Table IV shows the values of $T_{e}, n_{1}$ and degree of ionization at two radiofrequencies as a function of pressure. The electron temperature was found to be almost independent of the frequency, while the positive ion concentration was higher at a higher frequency. In consequence, there is an increase in the degree of ionization at the higher frequency. Since a higher degree of ionization induces a higher ion-molecule interaction, an increase in the decomposition of $\mathrm{SiCl}_{4}$ and deposition of $\mathrm{Si}$ is to be expected and was actually observed.

Avni et a1. (1983abc), Gr111 et a1. (1983) and Manory et a1. (1983) have , made mass spectrometric sampling of microwave plasmas of $\mathrm{SiCl}_{4}+\mathrm{H}_{2}+\mathrm{Ar}$ mixtures, which were characterlzed using the double floating probe system shown in figure 39. They recognized that the deposition rate of $S i$ is highest and the $\mathrm{Cl}$ contamination in the $\mathrm{Si}$ deposit the lowest where the $\mathrm{SiCl}_{4}$ enters the plasma. The free radicals from the plasma were adsorbed and stablilized on alumina and their concentrations were determined to be highest upstream of the plasma rather than downstream (fig. 40). Based on these observations the 
formation of polymerized silicon species by radicals and fon-molecule interactions was proposed:

$$
\begin{aligned}
& \mathrm{SiCl}_{2} \stackrel{\mathrm{SiCl}}{\longrightarrow} \quad \mathrm{Si}_{2} \mathrm{Cl}_{2-4}^{\cdot} \stackrel{\mathrm{SiCl}}{\longrightarrow} 4 \mathrm{Si}_{x} \mathrm{Cl}_{y}+\mathrm{Cl}_{2} \\
& \mathrm{SiCl}_{3}+\stackrel{\mathrm{SiCl}}{\longrightarrow} 4 \mathrm{Si}_{2} \mathrm{Cl}_{5}+\stackrel{\mathrm{SiCl}}{\longrightarrow} 4 \ldots \mathrm{Si}_{x} \mathrm{Cl}_{y}{ }^{+}+\mathrm{Cl}_{2}
\end{aligned}
$$

They, however, noticed a nonproportionality between the deposition process of Si and its concentration in the plasma at higher pressures and input powers. This, of course, could be attributed to the etchant properties of chlorine atoms and ions, and sputtering by $\mathrm{Ar}^{+}$ions.

\section{Insulating Films}

Insulating films are invariably compounds, usually oxides grown by plasma oxygenation of metals and carbonaceous materials containing $H$ or $F$ produced by glow discharge polymerization of hydrocarbons or fluorocarbons. 13 They have also been produced by glow discharge sputtering.

Glow discharge sputtering of compound targets has been studied by a number of authors. By using the ion sampling and energy analysis system that was previousiy described, Coburn et al. (1974) found that the intensity of sputtered neutral molecules exceeded that of sputtered neutral atoms for several oxide targets in an rf diode glow discharge. Given that a metal oxide target, and by implication any other compound target, is unlikely to be sputtered in a molecular form, it is not surprising that the stoichiometry of the resulting film will be different from that of the target, usually being deficient in the gaseous or other volatile species. However, Erskine and Cserhati (1978) have shown that the stoichlometry of a quartz $\left(\mathrm{StO}_{2}\right) \mathrm{film}$ is the same as the target if the sputtering is done in a mixture of 95 percent $\mathrm{Ar}$ and 5 percent $0_{2}$. Usualiy this involves some sort of reactive sputtering. This is the type of process in which a compound is synthesized by sputtering a target (e.g.. T1) in a reactive gas (e.g., $\mathrm{O}_{2}$ or $\mathrm{Ar}-\mathrm{O}_{2}$ mixtures) to form a compound $\left(\mathrm{T}_{2}\right)$. It is impossible for us to review the numerous reactive sputtering processes that have been described in the literature. We shall refer the reader to an extensive bibliography prepared by vossen and Cuomo (1978). At very low reactive gas partial pressure and high target sputtering rate, it is well established that virtually all of the compound synthesis occurs at the substrate and that the stoichlometry of the film depends on the relative rates of arrival at the substrate of metal vapor and reactive gas.

plasma oxidation/anodization. - The formation of an oxide on a metal or semiconductor surface immersed and floating in an oxygen plasma is called "plasma oxidation." When the growth is stimulated by applying a bias to the substrate so that the film surface potential is above the floating potential the process is called "plasma anodization." The topic has been reviewed by

13 According to Hauser (1977) and Hosokawa et al. (1981) carbon coatings deposited by sputtering are conducting rather than insulating. 
Dell'Oca et a1. (1971), O'Hanton (1977), Gourrier and Bacal (1981), Ojha (1982), and many others.

Earlier work on plasma oxidation by Ligenza (1965), Weinreich (1966) and others were done in microwave or dc discharges which were inadequate in producing pin hole free thin oxide films at controlled rates. Further, Lesile et al. (1978) have reported that the films produced in dc discharges were contaminated by the cathode material. Greiner $(1971,1974)$ was able to overcome these problems by placing the substrate to be oxidized on the cathode of an $r f$ system and adjusting the cathode potential to a value that resulted in simultaneous etching and oxidation by oxygen ions during the oxide growth. Ray and Reisman (1981) recorded the growth of $\mathrm{S10}_{2}$ only at pressures > 10 mtorr; the growth rate on the Si surface facing away from the discharge was significantly higher. Haper et al. (1981), however, reported that the rf oxidation technique suffers from such drawbacks as contamination of the oxide film due to sputtering of the walls, deposition of back-sputtered material, and excessive heating of the sample during oxidation. Since particle energies may reach as high as $10^{3} \mathrm{eV}$, some ion implantation is to be expected. Despite these problems, the rf process has become dominant for the fabrication of tunnel barriers for Josephson technology, for both lead alloy and niobium fllms.

A schematic representation of the physical processes occurring during plasma anodization is shown in figure 41. That the oxide growth rate and the oxide film thickness increases on application of a positive potential led 0 'Hanion and Pennebaker (1971) to suggest that negative oxygen ions in the discharge were essential for the oxide growth. However, 0live et al. (1972) found only a small decrease in the anodization rate when the barrier for the negative ion flux was increased by decreasing the dc surface potential of a tantalum substrate through the application of a combination of $\mathrm{dc}$ and $\mathrm{rf}$ potentials at constant anode current. Gourrier et al. (1980) found from photodetachment and probe measurements that the supply of negative ions from the plasma cannot account for the observed oxidation rates of GaAs. Although 0'Hanlon and Sampogna (1973) were able to increase the anodization rate by biasing positively an additional grid, their probe measurements indicated essentially that the electron temperature was enhanced. By using a magnetic field Chang (1979) prevented the electrons from reaching the plasma surface and found a strong reduction of the growth rate. Therefore, electron-assisted surface processes have been invoked. According to Adno and Matsumura (1978) electron capture by adsorbed oxygen atoms is the most likely process, the oxygen atoms originating from the plasma or on the surface by electronstimulated dissociation. The possibility of dissociative attachment to adsorbed oxygen molecules cannot be ruled out.

During plasma anodization charge transport occurs in the oxide: electrons and oxygen anions move inwards, and sample cations outwards. Only a few results for GaAs and Si have been reported on the ratio of anionic to cationic movements. By using $18_{0}$ as a marker Yamasakt and Sugano (1980) showed 'that the oxygen order is largely conserved during GaAs plasma anodization in an $r f$ discharge. This suggested that long-range migration of oxygen anions by direct interstitial transport is not dominant. Perriere et al. (1982) arrived at a similar conclusion from their 160 plasma anodization of $s i$ through thin 180 -enriched $\mathrm{ZrO}_{2}$ layers. By using double-layered structures of $\mathrm{Al} / \mathrm{GaAs}$ Chang (1979) and Gourrier et al. (1980) found that GaAs oxide can be formed on both sides of the $A l$ layer, which is itself oxidized, and concluded that both 
oxygen fons and substrate ions move during the anodization. However, Ho and Sugano $(1979,1982)$ and Chang et al. (1980) found that only oxygen migration occurs during si oxidation. Thus, the results appear to depend on the oxidized or anodized material. Fromhold (1977, 1982) and Fromhold and Baker (1980) have carried out calculations using a very simplified model which assumes that the rate of oxidation is limited by the transport of ionic species through the already formed layer, with the transport mechanism being the thermally activated hopping of ionic defects in the presence of electric fleids due to the surface potential established by the discharge and modified by the space charge of the mobile ionic defects. The results on the oxidation of InP which Meiners (1982) and Wager et al.(1982) have obtained recently using heated afterglows rather than plasmas seem to suggest a significant contribution from plasma excited neutrals as well.

Glow discharge nitriding. - Nearly half a century ago the use of a lowpressure glow discharge for nitriding was introduced by Berghouse (1932) and von Bosse et al. (1932). The material to be nitrided is used as the cathode in a glow discharge produced in a gas mixture which contains nitrogen. The gas mixtures used are $\mathrm{N}_{2}+\mathrm{Ar}, \mathrm{N}_{2}+\mathrm{H}_{2}, \mathrm{NH}_{3}+\mathrm{Ar}$, etc. For rapid nitride growth high current and power densities $\left(0.1\right.$ to $10 \mathrm{~mA} \mathrm{~cm}^{-2}$ and 0.5 to $\left.1 \mathrm{kV}\right)$ are desirable so that the abnormal region of the glow discharge is preferred. Besides steel, refractory metals such as $\mathrm{Zr}$, T1, Mo, Nb and carbides such as TiC have been nitrided in a glow discharge. Gruen et al. (1980), Veprek (1980) and 0jha (1982) have reviewed plasma nitriding in some detail.

Emission spectroscopy of glow discharges in $\mathrm{N}_{2}$ has shown the presence of $\mathrm{N}^{+}$and $\mathrm{N}_{2}{ }^{+}$so that the cathode undergoes energetic ion bombardment besides adsorbing neutral gas species such as atomic nitrogen. Because a steel specimen could not be nitrided in a floating position, Hudis (1973) concluded that adsorbed gas species are insufficient for nitriding. Edenhofer (1974) and Lakhtin et a1. (1976) observed that reactive sputtering of the cathode and back-diffusion of the sputtered species are responsible for the nitriding of steel. Sputtered Fe atoms react with nitrogen to form unstable FeN, which is back-scattered onto the cathode and decomposed to lower $n i-$ trides, such as $\mathrm{Fe}_{2} \mathrm{~N}, \mathrm{Fe}_{3} \mathrm{~N}$, and $\mathrm{Fe}_{4} \mathrm{~N}$. A fraction of the nitrogen atoms released due to such a decomposition is believed to diffuse into the steel.

Hudis (1973) found by mass spectrometric sampling of lons through a hole in the center of the cathode that in a nitrogen-hydrogen plasma nitrogen ions $\left(\mathrm{N}^{+}, \mathrm{N}_{2}^{+}\right.$, etc.) comprise less than 0.1 percent of the cathode current whtle nitrogen-hydrogen molecular ions comprise 10 to 20 percent. In a nitrogen-argon plasma, however, $\mathrm{N}^{+}$and $\mathrm{N}_{2}{ }^{+}$comprise 80 percent of the cathode current but produce very ittle nitriding. Nevertheless, a number of authors, among them Hudis (1973). Lebrun et a 1. (1972) and Content et a 1. (1974), suggested that fast fons and neutrals from the glow penetrate into the cathode to a depth of several atomic lengths and that the nitrogen absorbed in this way diffuses into the metal and forms a layer with a nitride structure. A nitriding mechanism based on the formation of vacancy and nitrogen ion pairs and itheir subsequent diffusion inside the metal was proposed by Brokman and Tuler (1981). They used crossed electric and magnetic fields to enhance the ion current density and found that the diffusion coefficient was proportional to the current density. However, Tibbetts (1974) found nitriding even when positive ions were repelled from a steel surface by a biased grid. 
Szabo and Withelmi (1983) have made a mass spectrometric study of continuous and pulsed dc discharges used for steel nitriding. They observed $\mathrm{NH}_{x}(x=0-4)$ and a strong cataphoretic enrichment of hydrogen. When a freshly nitrided steel surface was sputtered in $\mathrm{Ar}, \mathrm{Fe}, \mathrm{FeNH}_{2}$, and $\mathrm{FeNH}_{3}$ tons were detected. Therefore, they proposed the formation of a $\mathrm{FeNH}_{2}-3$ boundary layer. This is consistent with the work of Braganza et al. (1979) which showed that in a hydrogen glow discharge nitrided stainless steel is considerably depleted in nitrogen.

Liu et al. (1978) found that the kinetics of $\mathrm{Ti}$ and $\mathrm{Zr}$ nitride formation are enhanced by about a factor three in the dc discharge technique compared with thermal nitriding. Konuma and Matsumoto (1977). Matsumoto and Kanzaki (1983) and Matsumoto et a.1. (1982) have studied the nitriding of $\mathrm{Ti}$ and $\mathrm{Zr}$ in a nitrogen and 10 percent $\mathrm{H}_{2}$ to 90 percent $\mathrm{N}_{2}$ rf discharge at 5 to 20 torr pressure. Probe measurements revealed that the $\mathrm{N}_{2}$ and $\mathrm{N}_{2}+\mathrm{H}_{2}$ plasmas, respectively, had electron energies of 8 to 10 and 9 to $17 \mathrm{eV}$, and ion densities of $10^{9}-10^{10}$ and $10^{10} \mathrm{~cm}^{-3}$. From these values, $V_{f}$ was estimated as -80 to $-100 \mathrm{~V}$. Emission spectroscopy showed a number of electronic transitions, among them the $C^{3} \pi_{4}-B^{3} \pi_{g}$ transition of $N_{2}$, the $B^{2} \Sigma_{u}{ }^{+}-X^{2} \Sigma_{g}{ }^{+}$ transition of $\mathrm{N}_{2}{ }^{+}$and the $\mathrm{A}^{3} \pi-X^{3} \mathrm{E}^{2}$ - transition of $\mathrm{NH}$, the latter in the plasma which contained 10 percent $\mathrm{H}_{2}$ (table $\mathrm{V}$ ). The $\mathrm{N}_{2}{ }^{+}$was estimated to have a vibrational temperature of $5700 \mathrm{~K}$ for $\Delta V=1$. Besides $\mathrm{N}^{+}$and $\mathrm{N}_{2}^{+}$mass spectrometric sampling of the $\mathrm{N}_{2}+\mathrm{H}_{2}$ plasma while nitriding showed $\mathrm{NH}_{x}$ $(x=1$ to 4 ) species. Matsumoto and co-workers found that the addition of $\mathrm{H}_{2}$ decreased the concentration of $\mathrm{N}_{2}^{+}$and consequently the sputtering of Ti by $\mathrm{N}_{2}{ }^{+}$. Based on an ESCA analys is which revealed $\mathrm{NH}$ radicals on titanium surface Matsumoto proposed mechanisms consistent with an initial nitride forming reaction involving $\mathrm{NH}$ with the substrate, followed by the diffusion of nitrogen from nitride to metal.

The nitriding of sllicon in if plasma has been reported. By using a double probe Akashi et a1. (1981) found that the plasma (electron) density in $\mathrm{N}_{2}$-He discharge was much higher than those in $\mathrm{N}_{2}$ discharges and attributed the effective nitriding of $\mathrm{Si}$ in $\mathrm{N}_{2}$-He discharges to increased activation and ionization of $N_{2}$ by Penning effect of excited He. The SiN films were, however, uneven due perhaps to a radial distribution of $\mathrm{N}_{e}$ and $\mathrm{T}_{e}$ in the reactor tube. Matsumoto and Yatsuda (1981) used $\mathrm{N}_{2}$ and $\mathrm{N}_{2}-\mathrm{H}_{2}$ plasmas with electron densities of $10^{8}-10^{10} \mathrm{~cm}^{-3}$ and electron energies of 4 to 9 and 4 to $14 \mathrm{eV}$, respectively. The mass spectrometric samplings of their $\mathrm{N}_{2}$ plasma showed $\mathrm{N}^{+}, \mathrm{N}_{2}{ }^{+}$, $\mathrm{NO}$ and $\mathrm{Si}$; the addition of $\mathrm{H}_{2}$ suppressed $\mathrm{Si}$ and NO. The NO formation was explained by the sputtering of $\mathrm{SiO}_{2}$ from the discharge walls by $\mathrm{N}_{2}^{+}$and the reaction of $\mathrm{SiO}_{2}$. with $\mathrm{N}_{2}$ in the plasma. The hydrogen apparentiy suppressed $\mathrm{NO}$ formation and produced $\mathrm{NH}_{X}(x=1$ to 3$)$ which aided in the nitriding process. Evidence from ion beam studies of Taylor et al. (1978) suggests that $\mathrm{N}_{2}{ }^{+}$ions undergo charge exchange and $\mathrm{dis}^{\mathrm{s}-}$ sociate at the surface of silicon and its oxides $\left(S 10, S 10_{2}\right)$ to form hot $N$ atoms which react with $S i$ producing nitrides which are similar to those of the type $\mathrm{Si}_{3} \mathrm{~N}_{4}$.

Plasma carburizing. - In principle the method is similar to glow discharge nitriding. Grube and Gay (1978) studied the carburizing of a steel cathode in a dc cold cathode glow discharge of a hydrocarbon gas ( 1 to 20 torr) heated by an external furnace to a temperature of $1050^{\circ} \mathrm{C}$. Under the experimental conditions the hydrocarbon dissociates not only pyrolytically but also by electron bombardment in the discharge region, the latter enhancing the 
deposition rate of carbon on the metal surface. It has been suggested that high infusion rates of carbon into the surface, rather than high diffusion rates, are responsible for the high carburizing rates achieved in plasma carburizing.

Yoshihara et al. (1978) have reported the preparation of SiC films by carburization in an rf discharge of a $2: 3$ mixture of $\mathrm{SiH}_{4}$ and $\mathrm{CH}_{4}$ by volume. Konuma et a1. (1979) used an rf plasma in methane (10 to 30 torr) for carburizing of metals such as $\mathrm{Tl}, \mathrm{Zr}, \mathrm{Nb}$, and $\mathrm{Ta}$. Although the carbides were identified on the metal surface by ESCA analysis the discharge produced oily products which were identified by mass spectroscopy to be polyethylene and a mixture of higher chain hydrocarbons with mass numbers as high as 450 amu. Although these glow discharges have not been analyzed under carburtzing conditions, there is a considerable body of evidence from glow discharge polymerization work (see below) that $\mathrm{CH}_{4}$ radicals may be responsible for carbide formation.

Glow discharge polymerization. 14 - When a glow discharge is produced in the vapor of an organic compound or its mixture with a noble gas, such as Ar, films of a polymeric nature are deposited on the surfaces exposed to the glow/afterglow. The observation of this phenomenon dates back to de Wilde (1874) and Thenard and Thenard (1874). For nearly a century, however, such films were considered undesirable by-products and efforts were made to prevent film formation. For example, Suhr (1974) designed reactors with externally heated walls to prevent film formation. About the 1950's the advantageous features of these organic flims (e.g., flawless thin coatings, good adhesion to the substrate, chemical inertness, and low dielectric constant) were recognized. Since then much applied research on the use of the process has been done and several hundred papers published. It is impossible for us to review all this work here. The interested reader is referred to review articles by Kolotyrkin et al. (1967), Mearns (1969), M1llard (1974), Havens et a). (1976), Bell (1976, 1980), Yasuda (1978), Kay et a1. (1980), Shen and Bell (1979) and Boenig (1982). Gazicki and Yasuda (1983) have summarized the electrical properties of these fllms and their control by regulating the glow discharge parameters.

Besides hydrocarbons, organic compounds containing nitrogen, fluorine, oxygen and sliticon have been used as starting materials for glow discharge polymerization. The overall mechanism has been represented schematically (fig. 42) by Poll et al. (1976). It suggests that monomer can be converted into reactive and nonreactive products through processes occurring in the plasma (II and IV) as well as entering into polymer formation (I). The reactive products may also contribute to polymer deposition (III) or be converted to nonreactive products (V). The degradation of the polymer to form nonreactive products (VI) is included.

- ${ }^{14}$ In contrast to conventional polymerization - i.e.. molecular polymerization - polymer formation in glow discharge may be characterized as elemental or atomic polymerization. As Yasuda (1978) pointed out the term "plasma polymerization" should strictly be applied to polymerization which occurs in a plasma state. The term "glow discharge polymerization" has a wider meaning in the sense that includes plasma-induced polymerization. 
It should be noted that the predominance of a mechanism will depend not only on the chemlcal structure of the starting materials but also on the conditions of the discharge. The nature of the gaseous product(s) plays a.significant role in determining the extent of the ablation process. For example, Kay (1977) has reported that ablation of the polymer deposit occurs by shutting off the hydrogen which caused the deposition of polymer in an otherwise nonpolymerizing $\mathrm{CF}_{4}$ discharge. Evidently $\mathrm{H}_{2}$ removes $\mathrm{H}$ as $\mathrm{HF}$ from the discharge system, thus reducing the etching effect by the fluorine plasma. The importance of the ablation process is also demonstrated by the poor polymer formation reported by Yasuda (1977) in glow discharges of oxygen-containing compounds. In short, the deposition observed in glow discharges is the resultant of the competitive ablation and polymerization processes. Further, it is necessary to distinguish between rate of polymerization and rate of deposition. In the literature these two terms are used interchangeably; unfortunately, they do not represent the same thing.

Most of the published work is concerned with the deposition rate on processing factors such as the reactor geometry, discharge mode, frequency and power, gas pressure and flow rate of the organic compound and carrier gas, if any. While high deposition rates result from the imposition of a magnetic fteld (Morosoff et al. 1978), pulsed discharges reduce the rate of polymer formation considerably (Yasuda and Hsu, 1977). Both Yasuda (1978) and Boenig (1982) have well summarized the effect of these external parameters on $\mathrm{f} 11 \mathrm{~m}$ deposition.

Diagnostics of polymerizing plasma was reported by Nilnomi and Yanagihara (1979), Yanagthara et a1. (1981), and Yanagthara and Nitinomi (1983) who measured $\mathrm{T}_{e}, \mathrm{n}_{e}$ and $\mathrm{f}(\mathrm{E})$ for an $\mathrm{rf}$ plasma sustained in an $\mathrm{Ar} / \mathrm{C}_{6} \mathrm{H}_{6}$ mixture using heated probes. Under conditions of good quality film deposition, they found that $f(E)$ is non-Maxwellian (flg. 43). Unilke in pure Ar or $\mathrm{C}_{6} \mathrm{H}_{6}$ vapor the value of $\mathrm{n}_{e}$ decreased by four decades when a small amount of $\mathrm{C}_{6} \mathrm{H}_{6}$ (100 to $200 \mathrm{SCCM}$ ) was added to the Ar plasma; however, $\mathrm{n}_{e}$ increased by three decades with increasing benzene flow rate (fig. 44). The electron temperature ( $\mathrm{fig} .45$ ) showed a similar flow rate dependency to that of $\mathrm{n}_{\mathrm{e}}$, although not on a logarithmic scale. The reaction conditions which deposited good quality polymer film usually corresponded to a plateau region of low pressure ( 0.5 torr) and high benzene flow rate (500 SCCM) and $\mathrm{n}_{\mathrm{e}}$ and $\mathrm{T}_{\mathrm{e}}$ values of $2.25 \times 10^{9} \mathrm{~cm}^{-3}$ and $4.25 \times 10^{4} \mathrm{~K}$, respectively. The reaction conditions for the lowest value of $n_{e}$ and $T_{e}$ corresponded to those producing a lot of powder.

Nilnomi and Yanagihara (1979) monitored the concentration of positive ions and neutrals under the above conditions. The mass spectrum of the positive plasma ions exhibited almost the same trends as the mass spectrum of neutral species present in the plasma ( $\mathrm{flg} .46)$ and the pyrolys is mass spectrum of plasma-polymerized benzene reported by Venugopalan et a1. (1980). Principal plasma lons assigned from the observed m/e values are 11 sted in table VI. Although the mechanism of the formation of the observed ionic ispecies is uncertain, the formula of $C_{7}$ to $C_{12}$ oligomers (the second column, coded as 1 st additives) can be made up by simply adding $\mathrm{C}_{6} \mathrm{H}_{6}$ to corresponding fragment formula, which was found in abundance in the plasma, e.g.,

$$
\mathrm{C}_{6} \mathrm{H}_{5}^{+}+\mathrm{C}_{6} \mathrm{H}_{6} \rightarrow \mathrm{C}_{72} \mathrm{H}_{71}+
$$


For oligomers of $C_{13}$ to $C_{18}$ as seen in the third column, a simliar ionmolecule reaction mechanism appears to be valid. For the higher homologs, however, hydrogen loss seems to occur for structural stabliization. Although ion-molecule reactions play a role in plasma polymerization (in the gas phase), there is no evidence that the mechanism of the formation of thin polymer film of a substrate is the same as that occurs in the gas phase.

Yanagthara et a1. (1982a,b) and Yanagihara and Yasuda (1982) have applied similar diagnostic techniques to $\mathrm{rf}$ glow discharges in $\mathrm{CH}_{4}, \mathrm{C}_{2} \mathrm{H}_{4}, \mathrm{CF}_{4}$, and $\mathrm{C}_{2} \mathrm{~F}_{4}$.

In an rf discharge in methane Smolinsky and Vasile (1973, 1975) and Vasile and Smolinsky $(1973,1975)$ found that polymer deposits were formed more rapidly on the electrodes than on the walls and they detected a greater number of one and two carbon ions, espectally $\mathrm{C}_{2} \mathrm{H}_{3}^{+}, \mathrm{C}_{2} \mathrm{H}_{2}^{+}, \mathrm{CH}_{3}^{+}, \mathrm{CH}_{2}^{+}$, and $\mathrm{CH}^{+}$, in the dark space adjacent to the electrodes than in the space adjacent to the walls. Since the neutral $\mathrm{C}_{2} \mathrm{H}_{4}$ and $\mathrm{C}_{2} \mathrm{H}_{2}$ did not influence the rate they concluded that ions arriving on the surface are important than neutrals in terms of the rate of polymerization. Following later works on extraction of positive ions from rf plasma in $\mathrm{C}_{2} \mathrm{H}_{4}$ (Smolinsky and Vasile, 1976) and in $\mathrm{C}_{2} \mathrm{H}_{2}$ (Vasfle and Smolinsky, 1977) they concluded that the polymerization in hydrocarbon plasmas is propagated by positive ions. This is in agreement with the earlier work of Westwood (1971) and Thompson and Mayhan (1972), and the recent work of Khait et al. (1980) which showed that the deposition rate on a biased electrode in the plasma is nearly linearly proportional to the blasing voltage, indicating that the nature of the deposition by polymerization is dependent upon charge carriers.

Inspektor et a). (1979, 1981) and Carmi et al. (1981) have made a deta11ed analysis of microwave cavity plasmas of methane, propylene and the 1 m $m i x$ tures with argon in which pyrocarbon is deposited. They used three sampling and probe positions ( $\mathrm{fig}$. 39) which were located immediately before the cavity $(H)$, in the center of the cavity $(G)$, and immediately beyond the cavity ( $F)$. By using a double floating probe system (DEPS) they determined $\mathrm{T}_{e}, \mathrm{n}_{e}$, and $n_{i}$ at these locations. With respect to position $F$, the addition of Ar to $\mathrm{CH}_{4}$ increased $\mathrm{T}_{\mathrm{e}}$ (fig. 47) suggesting that an "heating effect" was caused. by $\mathrm{Ar}$ which is a better heat conductor than $\mathrm{CH}_{4}$. Also at this position the values of $n_{j}$ and $n_{e}$ in the plasma of $A r+\mathrm{CH}_{4}$ mixture were higher than the values for the $\mathrm{CH}_{4}$ plasma alone. The saturation currents of the positive ions on both probes and the electron current density increased with increasing $\mathrm{Ar} / \mathrm{CH}_{4}$ ratio and was attributed to the charge transfer reaction from $\mathrm{Ar}$ to $\mathrm{CH}_{4}$ :

$$
\begin{aligned}
& \mathrm{Ar}++\mathrm{CH}_{4} \rightarrow \mathrm{Ar}+\mathrm{CH}_{4}^{+} ; \Delta \mathrm{H}_{\mathrm{f}}=-2.78^{\circ} \mathrm{eV} ; \sigma=2 \times 10^{-15} \mathrm{~cm}^{2} \\
& \mathrm{Ar}^{+}+\mathrm{CH}_{4} \rightarrow \mathrm{Ar}+\mathrm{CH}_{3}^{+}+\mathrm{H} ; \Delta \mathrm{H}_{\mathrm{f}}=-1.36 \mathrm{eV} ; \sigma=1.04 \times 10^{-14} \mathrm{~cm}^{2}
\end{aligned}
$$

According to Inspektor et al. (1981) the values of $n_{i}$ and $n_{e}$ in a 'pure methane (no Ar added) increased in going from position $H$ to $G$, but decreased at position $F$ as pressure or power was increased (figs. 48 to 49 ). This is to be compared with the values of $n_{1}$ and $n_{e}$ with added Ar shown in figure 50. By using three different techniques, viz., probe (DFPS), Stark 
broadening and electric conductivity 75 at position $\mathrm{G}$ in $\mathrm{Ar} / \mathrm{C}_{3} \mathrm{H}_{6}$ mixture Inspektor et a1. (1981) found that the value of $n_{e}$ increased with increasing pressure. They also noted that for a given pressure the value of $n_{e}$ depended on the dlagnostic technique and could be different by as much as an order of magnitude (table VII). In view of this the discrepancies between $n_{j}$ and $n_{e}$ values in figures 48 to 50 were considered to originate from different diagnostic techniques and ignored. It was concluded that the decrease of $n_{1}$ and $n_{e}$ in going from position $G$ to $F$ was due to the formation of highly unsaturated hydrocarbon polymer lons which are deposited on the walls and lose their charge.

The results of mass spectrometric sampling of a propylene - argon plasma by Carmi et a1. (1981) is shown in figure 51. Note the nearly linear decrease of $\left[\mathrm{C}_{3} \mathrm{H}_{6}\right]$ along the reactor, 1.e., the reaction time in the flowing plasma which is representative of a first-order kinetics in the plasma. It was suggested that the reaction is initiated by ionization of $\mathrm{C}_{3} \mathrm{H}_{6}$ by either electrons, $\mathrm{Ar}^{+}$or metastable Ar:

$$
\mathrm{C}_{3} \mathrm{H}_{6} \stackrel{\mathrm{e}, \mathrm{Ar}^{+}, \mathrm{Ar}^{\star}}{\longrightarrow} \mathrm{C}_{3} \mathrm{H}_{6}^{+}
$$

Since $\mathrm{C}_{3} \mathrm{H}_{6}$ is always the species in great excess, the following propylene chain was proposed:

$$
\mathrm{C}_{3} \mathrm{H}_{6}+\stackrel{\mathrm{C}_{3} \mathrm{H}_{6}}{\rightarrow} \mathrm{C}_{6} \mathrm{H}_{10-6}^{+} \stackrel{\mathrm{C}_{3} \mathrm{H}_{6}}{\rightarrow} \mathrm{C}_{9} \mathrm{H}_{14-9} \stackrel{\mathrm{C}_{3} \mathrm{H}_{6}}{\rightarrow} \ldots \mathrm{C}_{p} \mathrm{H}_{q}^{+} \mathrm{p}>\mathrm{q}
$$

Fragmentation of the $\mathrm{C}_{3} \mathrm{H}_{6}+$ ion to $\mathrm{C}_{2} \mathrm{H}_{2}+$ rather than $\mathrm{CH}_{4}{ }^{+}$is thermodynamically favored and supported by mass spectrometric data. Therefore, an acetylene chain was also proposed:

$$
\mathrm{C}_{2} \mathrm{H}_{2}+\stackrel{\mathrm{C}_{3} \mathrm{H}_{6}}{\longrightarrow} \mathrm{C}_{5} \mathrm{H}_{4-6}^{+} \stackrel{\mathrm{C}_{3} \mathrm{H}_{6}}{\longrightarrow} \cdots \mathrm{C}_{\mathrm{r}} \mathrm{H}_{s}^{+} \quad r>s
$$

Since ethylene is produced in high concentration the following ethylene chain was also included:

$$
\mathrm{C}_{2} \mathrm{H}_{4}^{+} \stackrel{\mathrm{C}_{3} \mathrm{H}_{6}}{\rightarrow} \mathrm{C}_{5} \mathrm{H}_{5-8}^{+} \stackrel{\mathrm{C}_{3} \mathrm{H}_{6}}{\rightarrow} \ldots \mathrm{C}_{\mathrm{u}} \mathrm{H}_{\mathrm{r}} \quad \mathrm{u}>\mathrm{r}
$$

All three chain reactions were proposed to proceed in parallel and result in pyrocarbon deposits.

15If we assume that the current in the microwave plasma is carried , mainiy by electrons and consider only the dc component of the electric field strength, $E$, the electron conductivity $\sigma_{e}=e_{\mu} e_{e}$ where $\sigma_{e}$ is in $\Omega^{-1} \mathrm{~cm}^{-1}$. The electron density is directly evaluated from the measured electron current density $J_{e}$ by $n_{e}=\left(J_{e} / E\right) e_{e}$, where $E$ is in $V$ $\mathrm{cm}^{-1}$ and $j_{e}$ is given in $\mathrm{A} \mathrm{cm}^{-2}$. 
Inspektor et al. (1981) defined a degree of polymerization $\left(P_{m}\right)$ as the normalized sum of peak intensities $I_{X}$ of the high pyrocarbons, each multiplied by its carbon content $x$ :

$$
P_{m}=(1 / 3) \sum x \Sigma I_{x} / I_{C_{3} H_{6}} \quad x>3
$$

The variation of $P_{m}$ with and without argon is shown in figure 52 as a function of pressure. The polymerization is higher in the afterglow ( $F$ ) rather than in the foreglow $(H)$ and is decreased in the afterglow by an increase in pressure and/or the addition of argon. These results are consistent with the measured decreases in $n_{e}$ and $n_{i}$ in the afterglow (fig. 50).

Besides chain propagation by cationic species free radical polymerization mechanisms have been proposed. The basis for this is the presence of unpaired spins which has been reported by a number of authors who studied ESR spectroscopy of the plasma-polymerized fllms. If we assume that the trapped radicals in the fllms are related to the free radicals produced in the plasma it is eastly recognized that free radical concentrations in the plasma are $10^{3}$ to $10^{5}$ higher than the ion concentrations. Further, Morosoff et al. (1976) have observed higher radical concentrations at the surfaces of polymers exposed to a plasma. Surface free radicals are known to react with gas-phase free radicals and unsaturated molecules such as ethylene and propylene. Since these evidences are not derived directly from the analys is of glow discharge we shall simply refer the reader to articles in which Bell (1976, 1980) relates the rate at which polymer is formed with the experimentally controlled variables in glow discharge polymerization using free radicals as the primary species propagating chain growth, both in the gas phase and on the surface of the deposited polymer. Vinogradov et al. (1981) has discussed an "activation growth model" for the formation of $\left(\mathrm{CH}_{x}\right)_{n}$ polymer in $\mathrm{CH}_{4}$ discharges taking the radicals as bullding blocks whlle the charged particles promote the activation of the surface sites for the growing polymer film. The exact polymer deposition mechanism can only be delineated by experiments yet to be performed, in which either the ions or the radicals formed can be isolated from the substrate surface where deposition takes place.

Both saturated and unsaturated fluorocarbons deposit fluorine-containing flims in glow discharges. The general conclusion from ESCA analysis of numerous deposits is that plasma species from saturated fluorine compounds react mostiy with the surface creating. a somewhat permanently attached fluorinated layer. Unsaturated fluorocarbons, on the other hand, readily polymerize in a glow discharge, the main result being deposition of polymeric materials and not their grafting. Both neutral and ionic species have been monitored by Kay et al. (1976) and by Vasile and Smolinsky (1977) using in situ mass spectrometric analysis of $\mathrm{C}_{2} \mathrm{~F}_{4}$ glow discharges. In contrast to hydrocarbon systems, particularly $\mathrm{C}_{2} \mathrm{H}_{4}, \mathrm{C}_{2} \mathrm{~F}_{4}$ discharges produced a high yield of gaseous products as well as polymer deposits. Further, the intensities of the signals relating to unsaturated species are directly relatied to the polymer deposition rate (fig. 53). Millard and Kay (1982) have determined that the polymerization rate in a $C_{3} F_{8}$ plasma is related to the intensity of the $\mathrm{CF}_{2}$ band $(265 \mathrm{~nm})(\mathrm{fig} .54)$. Since the presence of $\left(C_{2}\right)_{n}$ species was observed in the effluents of plasmas excited in several different fluorocarbons and their gas phase concentration was directly related to the deposition rate, Kay et a1. (1978, 1980) and Kay and Dilks (1979, 1981) 
proposed that oligomerization of $\mathrm{CF}_{2}$ may occur to form $\mathrm{C}_{2} \mathrm{~F}_{4}, \mathrm{C}_{3} \mathrm{~F}_{6}$, $\mathrm{C}_{4} \mathrm{~F}_{8}$, etc. Evidently the primary precursors to polymerization have the general formula $\left(C_{2}\right)_{n}$ which may include both cyclic alkanes and monoolefins. Since the deposited $f i l m$ has $F / C<2$, the plasma species such as photons, lons and metastables must interact with the polymer to eliminate $F$ and even small fragments by ablation.

Cramarossa et a1. (1983), d'Agostino (1983) and d'Agostino et al. (1982, 1983) have used actinometric emission spectroscopy and mass spectrometry to analyze $r f$ discharges in several fluorocarbons and their mixtures with $\mathrm{H}_{2}$ or $0_{2}$, the latter because of their usefulness in plasma etching. They related emission intensities of both $\mathrm{Ar}$ and $\mathrm{N}_{2}$ actinometers to the electron excitation functions $f\left(n_{e}\right)$, a function of the electron densities $n_{e}$ at energles $E>E_{t h}$ (where $E_{\text {th }}$ is 11.5 and $13.7 \mathrm{eV}$ for $\mathrm{N}_{2}$ and $A r$, respectively). Table VIII gives the normalized value of $f\left(n_{e}\right)$, the measured radical densities and polymer deposition rates. They explained the polymerization process on the basis of a mechanism which involves $\mathrm{CF}$ and $\mathrm{CF}_{2}$ radicals as bullding blocks as well as an activation of the polymer surface by means of charged particle bombardment.

The deposition processes occurring in a fluorocarbon plasma are critically dependent upon the effective $F / C$ ratio, low ratios $(<2$ to 3 depending on the bias) favoring polymerization and high ratios ( $>2$ to 3 depending on the bias) favoring etching. Kay et al. (1978) and Kay and Dilks (1979) have combined the processes of etching and polymerization for producing metal containing fluorocarbon polymer deposits. The films were prepared using a mixture of argon and perfluoropropane $\left(\mathrm{C}_{3} \mathrm{~F}_{8}\right)$ in a capacitively coupled diode reactor in which the substrate was mounted on the grounded electrode. Germantum, molybdenum and copper were used for the rf powered electrode which at $13.65 \mathrm{MHz}$ attained an overall negative potential due to the greater mobility of the electrons than the lons in the plasma and functioned as the cathode. Therefore, the positive ions arrive at the cathode with increased kinetic energy and cathode material is removed from its surface by competitive physical sputtering in which momentum transfer to the surface is involved, as well as, chemical plasma etching through the formation of volatile species which subsequently desorb and enter the gas phase. However, copper is a typical example of a material which forms nonvolatile fluorides, therefore, in this case material can be removed only by physical sputtering. Indeed, mass spectra of the plasma effluents showed that the relative concentration of $\left(\mathrm{CF}_{2}\right)_{n}$ in the gas phase is directly proportional to the polymer deposition rate which is in the decreasing order $\mathrm{Ge}>\mathrm{Mo} \gg \mathrm{Cu}$. This order also reflects the efficiencles of the cathode etching process to lower the $F / C$ ratio in the plasma and, thus, increase the concentration of unsaturated species and therefore the polymerization rate.

Recently, Kay and Hecq (1983) have used both mass spectrometry and optical emission for the diagnostics of $\mathrm{a}_{3} \mathrm{~F}_{8} / \mathrm{Au}$ system in which the deposition rate was simultaneously measured by a quartz crystal microbalance is a function of different $\mathrm{Ar} / \mathrm{C}_{3} \mathrm{~F}_{8}$ mixtures. Their data (shown in $\mathrm{fig}$. 55) indicates that the deposition rate is nearly constant up to $\mathrm{CF}_{3}{ }^{+}$I $\mathrm{Ar}^{+} \leq 2 \times 10^{-4}$. Also, the normalized Au optical emission intensity paralleled the deposition rate curve. However, the $\mathrm{CF}_{2}$ emission starts when the deposition rate drops suggesting that the decrease in gold deposition is due to the onset of polymer formation on the $\mathrm{Au}$ cathode. 
Plasma-activated chemical deposition. - Films have been deposited by initiating chemical reactions in a gas with an electrical discharge. The technique is often referred to as plasma-activated (or plasma-assisted) chemical vapor deposition (PCVD). Such flims are typically the carbides, nitrides, oxłdes and oxynitrides of elements such as AT, B, Ge, S1, and Ti. Empirical descriptions of processes and properties of films are to be found in articles by Kirk (1974), Hollahan and Rosler (1978), Rand (1979), Reinberg (1979), Veprek (1980), 0jha (1982) and Bonifield (1982). Table IX is a representative, but not all-inclusive, list of the films produced and the starting materials used in $\mathrm{rf} / \mathrm{microwave}$ discharges. The cited references are also good sources of information on the correlation between deposition and properties of the films and the macrovariables of the glow discharges. Practically none of these glow discharges have been analyzed for understanding the mechanisms of $\mathrm{f} 1 \mathrm{~lm}$ deposition.

There have been few diagnostic investigations of glow discharges used in PCVD work. For example, Haque et al. (1975) used in situ mass spectrometry to study chemical transport in a carbon deposition system involving $\mathrm{CO}$ and $\mathrm{CO}_{2}$, Veprek et al. (1975) used matrix-1solation spectroscopy to study a carbon deposition system of hydrocarbons, Yoshihara et al. (1981) made a mass spectral analysis of a $\mathrm{SiH}_{4} / \mathrm{CH}_{4}$ system used for SiC deposition, and Ron et al. (1983) applied ESR spectroscopy to conclude that a radical mechanism governs the dissoclation of $\mathrm{SiCl}_{4}$ and $\mathrm{NH}_{3}$ in the formation of $\mathrm{Si}_{3} \mathrm{~N}_{4}$. Based on such 11 mited studies PCVD processes have been described thus far as chemical vapor deposition by free radicals produced in the glow discharge. Clearly. for each of the systems 11sted in table IX investigations are badly needed to elucidate the role of the glow discharge electrons, lons, photons, and other excited species in the deposition process.

\section{Proposed Models}

The heterogeneous reactions such as boriding, carburizing and nitriding of the respective atoms, molecules, or fons with metal surfaces are mainiy exothermic. This is substantiated by Samsonov and Vinitskif's (1980) data for the Gibbs energy change $(\Delta G)$, which are collated in table $X$. The computations, however, assume that the boron, nitrogen, and carbon were supplied as atoms to the metal surface. In a glow discharge, the dissociation of $\mathrm{B}_{2} \mathrm{H}_{6}$ or $\mathrm{BC}_{3}, \mathrm{~N}_{2}$ or $\mathrm{NH}_{3}$, or hydrocarbon gases to $\mathrm{B}, \mathrm{N}$ or $\mathrm{C}$ atoms, respectively, takes place with a certain degree of atomization or ionization, both being functions of the macro and micro variables of the plasma. From the thermodynamic point of view, the interaction between atoms and/or ions with the metal surface results in chemical compounds, the extent of their deposition, that is, thickness beneath the surface, being diffusion-controlled. Figure 56 shows the various chemical compounds, such as oxides, borides, carbides and sllicides, which were detected on 440-C steels by Brainard and Wheeler (1978) using the XPS technique. The 440-C steels were preoxidized to form a Spinel interface $\left(\mathrm{FeO}_{-} \mathrm{Fe}_{2} \mathrm{O}_{3}\right)$ on which $\mathrm{Mo}_{2} \mathrm{~B}_{5}, \mathrm{Mo}_{2} \mathrm{C}$ and $\mathrm{MoSi}_{2}$ were 'deposited by $\mathrm{rf}$ sputtering. A review of the literature, for example, the papers by Avni (1984), Mattox (1982), Thornton (1982), etc., however, indicates that not enough data are avallable to conclude that the adherence of a deposited film on a surface is controlled by a chemical bond rather than a van der Waals bond. 
Film deposition in a glow discharge system is a dynamic, irreversible, kinetic process which begins with homogeneous reactions in both plasma bulk $(\mathrm{PB})$ and layer (PL) and terminates through heterogeneous reactions in the neutralization layer (NL). Table XI lists the various kinetic processes leading to the formation of the coating fllms. The rate-determining step in this dynamic deposition process is probably controlled by the heterogeneous plasma surface interactions (PSI). Before describing the various kinetic models it is worthwhile to emphasize the irreversiblility of the process, that is to say, a solid product is obtained from a gas monomer supplied to the plasma.

Consider the plasma process in which solid $\mu \mathrm{CS} 1$ is deposited on a substrate from the monomer of tetrachlorosilane vapor:

$$
\mathrm{SiCl}_{4}(\mathrm{~g})+\mathrm{Ar}+\mathrm{H}_{2} \longrightarrow \frac{\mathrm{Plasma}}{\longrightarrow} \quad \mu \mathrm{CSI}: \mathrm{H}: \mathrm{Cl}(2)+\mathrm{HCl}(\mathrm{g})+\mathrm{Ar}
$$

Two main processes may contribute to the reversibility of the reaction, viz., the formation of tetrachlorosilane gas from solid microcrystaliine silicon:

(i) Sputtering of $\mathrm{Si}$ atoms or clusters by $\mathrm{Ar}^{+}$bombardment; to the PL.

(i1) Plasma etching by hydrogen and chlorine, releasing chlorosilane back

The calculated and measured values of fluxes and rates of sputtering, etching and deposition are given in table XII for $\mathrm{Ar}^{+}, \mathrm{Cl}_{2}$ and $\mathrm{F}_{2}$. The rate of $\mu \mathrm{CSi}$ deposition is higher by more than one order of magnitude of the combined rates of sputtering and chemical etching. This has been taken as evidence of a low probablitity for reversible reactions at pressures above 0.5 torr in an rf discharge.

Tibbit et al. (1977) have proposed reinitiation processes based on earlier models by Denaro et al. (1968) and Poll et al. (1976), in which the electrons, radiation and fon fluxes of the plasma reactivate the deposited polymer to form active radicals on the surface which, in turn, may react with the incoming gas particles, such as fresh monomer, its fragments or polymertzed molecules. These reactions release what Poll et al. (1976) termed "nonreactive reaction products, "such as $\mathrm{H}_{2}, \mathrm{Cl}_{2}$, etc. In these reinitiation processes the irreversibility described above is still maintained.

Denaro et a1. $(1968,1969,1970)$, Poll et al. (1976), Lam et a1. (1976) and Tibbitt et a1. (1977) have proposed theoretical models for evaluating the deposition rate in plasma polymerization processes. Bell (1980) has reviewed these models which are based on the following observations and assumptions:

(1) The propagation of homogeneous and heterogeneous polymerization is promoted mainly by radical reactions which are listed in table XIII.

1. (2) The rate of polymer deposition is expressed in terms of the rate of monomer consumed.

(3) The extent of gas phase termination in the plasma system is very small and essentially all of the radicals formed in the plasma state are adsorbed on the deposited polymer surface. 
(4) The concentrations of adsorbed monomer and free radicals are proportional to the gas phase concentrations of these species.

(5) The rate of hydrogen atoms formed (hydrocarbon systems) is equivalent to the rate of free radicals produced.

Tibbits et al. (1977) gave the following analytical expression for the polymer deposition rate, dG/dt:

$$
(d G / d t)=[2 c a /(a-b)]\left[M_{g}\right]_{\delta}^{2}[(1-\exp [-(a+b) \tau]) / \exp [(a+b) \tau]]
$$

With reference to table XIII, we write

$$
\begin{gathered}
a=k_{1}[e]=k_{1}[e]=k_{2}[e]=k_{4}[e]=2.1 \times 10^{-2} \mathrm{~s}^{-1} ; \\
b=(2 / L) k_{a} ; k_{a}=k_{8}[S]=7.5 \times 10^{-3} \mathrm{~cm} \mathrm{~s}^{-1} ; \\
c=(L / 2)\left(k_{p g}+k_{p s}\right) ; k_{p s}=k_{10} k_{R}=5.5 \times 10^{-18} \mathrm{~cm}^{4} \mathrm{~s}^{-1} ; \\
k_{p g}=k_{5}=k_{6}=3.5 \times 10^{-18} \mathrm{~cm}^{3} \mathrm{~s}^{-1} ; .
\end{gathered}
$$

$\tau=V / Q$, where $V$ is the plasma volume, $Q$ is the flow rate, and $L$ is the inter-electrode separation. The quality of the fit of equation (62) to experimental data is shown in figure 57 for a number of hydrocarbons. Since the calculated values agree very well with the experimental results, a discussion of the assumptions in arriving at equation (62) is given below.

Homogeneous and heterogeneous propagation. - Two types of reactions, viz., positive ion - molecule reactions and radical - molecule reactions have been discussed in connection with mechanisms in plasma systems of flowing gases. Which of these is rate-determining in homogeneous and heterogeneous processes in a given plasma system has not yet been delineated.

Avni (1984) and Avni et a). (1983) have introduced the concept according to which free radicals, positive and negative ions are formed continuously along $Z$ in the plasma as a result of the interactions of the monomer gas with energetic electrons of the plasma. Therefore, the concentration per unit volume $\left(\mathrm{cm}^{-3}\right)$ of free radicals, $n_{R}$, positive lons, $n_{j}$, and negative lons, $n_{N}$, and their spatial gradients are variables which must be considered in the kinetics of the reactions with monomer molecules. Other variables controlling the kinetics is the reactivity of the radicals and ions, i.e., the cross sections $\sigma_{R}, \sigma_{j}$, and $\sigma_{N}$ of these reactions. The probability $P$ of a reaction is the product of $n$ and $\sigma$ in a given location $z$ along the plasma. It follows that; if

$$
n_{R} \times \sigma_{R}>n_{i} \times \sigma_{i} \text {, then } P_{R}>P_{i}
$$

1.e., the probability of radical - molecule interactions is higher than the probability of ion-molecule reactions. Conversely,

$$
n_{1} \times \sigma_{1}>n_{R} \times \sigma_{R} \text {, then } P_{1}>P_{R}
$$

Bel1 (1980) has suggested a value of $10^{-18} \mathrm{~cm}^{3}$ molecule-1 $\mathrm{s}^{-1}$ for the rate constant for radical - monomer interactions, such as reactions (5) and (6) in table XIII. According to Chatham et al. (1983) ion-molecule charge transfer reaction rates are typically of the order of $10^{-10_{-}} 10^{-12}$ 
$\mathrm{cm}^{3}$ molecule $\mathrm{s}^{-1}$. Thus, for concentrations $\mathrm{n}_{R}=10^{3} \mathrm{n}_{1}, \mathrm{P}_{\mathfrak{j}}>\mathrm{P}_{\mathrm{R}}$. Avni et a 1. (1983, 1984) have shown that for the polymerization of hydrocarbons $P_{1}>P_{R}$ in $r f$ plasmas with Ar, whlle $P_{R} \geq P_{1}$ in $r f$ plasmas without Ar. The same type of behavior was reported by Avni et al. $(1983,1984)$ for $\mathrm{SiCl}_{4}+$ $\operatorname{Ar}\left(P_{1}>P_{R}\right)$ and for $\mathrm{SiCl}_{4}+H_{2}+\operatorname{Ar}\left(P_{R} \geq P_{j}\right)$ rf plasma systems. Thus, depending on the plasma system etther $P_{R} \geq P_{1}$ or $P_{1} \geq P_{R}$ can be selected and controlled. Such a selection, however, is not feasible for the theoretical condition where $P_{R}=P_{i}$.

Deposition Rate Expressed as the Rate of Monomer Consumed. - This assumption has been investigated by Avni et al. (1983, 1984) using mass spectrometric sampling at different locations along the plasma. An overall reaction rate, $k_{0}$, for the dissociation of the monomer $(M), I_{M} / \Sigma I$, and the formation of products was evaluated from data such as those shown in figure 51 for a $\mathrm{C}_{3} \mathrm{H}_{6}+\mathrm{Ar}$ plasma and in figure 58 for a $\mathrm{SiCl}_{4}+\mathrm{Ar}$ plasma with and without $\mathrm{H}_{2}$. Because of the large excess of monomer in plasma chemical reactions of the type

$$
M+s(+)(\cdot) \rightarrow \text { Products }
$$

where $s(+)(\cdot)$ is some spectes in the plasma (ion or radical), were. considered to be pseudo-first-order in $M$ and $S$. Since at all positions ( $H, G$, and $F$ in fig. 39) in the plasma steady-state conditions exist and the flow velocities between $H$ and $G$ and $G$ and $F$ are known, the conventional kinetic equation applies:

$$
\int_{\tau_{0}}^{\tau_{0}} \text { at }{ }_{H} \text { at } \ln \left(I_{M} / \Sigma I\right)=k_{0} \tau_{0}
$$

Therefore, the value of $k_{0}$ could be evaluated from the slope of the plot of $I_{M} / \Sigma I$ versus sampling position shown in figures 51 and 58 , and the values of $\tau_{0}$ for the distances $H-G$ and $G-F$ at a given flow rate and pressure. Table XIV lists the calculated values of $k_{0}$ for the dissoctation of $\mathrm{C}_{3} \mathrm{H}_{6}, \mathrm{SiH}_{4}$ and $\mathrm{SiCl}_{4}$.

Avnl et al. (1983) used a different model for species $S$ which were short-lived in the plasma. This allowed the calculation of a local rate constant, $K_{L}$, using the equation:

$$
k_{L} \tau_{L} P=\ln (I / \Sigma I)
$$

where

$\tau L$ ion or radical residence time

$P$ reactant partial pressure.

The ionic residence time was evaluated by using its moblitity and the Langevin relationship, whlle the radical residence time was found from its velocity and collision frequency. By making use of equations (65) and (66) the monomer consumption in the plasma, $\mathrm{dM} / \mathrm{dt}$, could be calculated and related to the polymer deposition rate, $d G / d t$. Representative data are given in table XV for 


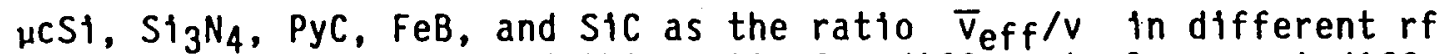
plasmas. The constancy of this ratio for different plasmas at different operating conditions verifies the assumption and allows dM/dt to be correlated with $d G / d t$.

Equation (17) gives the rate of fonization and excitation in the plasma layer (PL). When compared with phenomena in the plasma bulk (PB) there are strongly enhanced rates of excitation and ionization (fig. 15) in the PL due to the secondary electrons (HEEB). The flux of HEEB, jes, is given by Khatt et a1. (1980) as

$$
j_{e s}=r\left(c_{1 s}\right) j_{i s}=(1 / 4) y a_{i} n u_{i}
$$

where

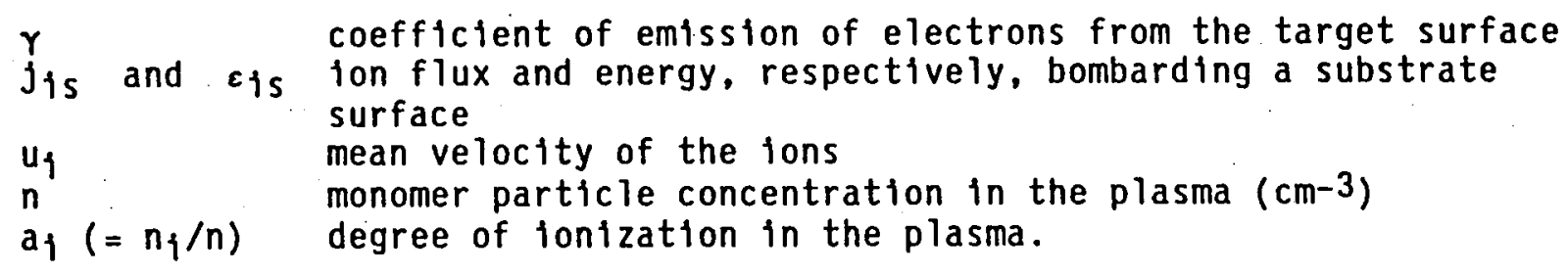

By introducing equation (67) into equation (17), Khait et al. (1980) showed that

$$
d n_{1} * / d t=\bar{v} n s\left(1+\left[b_{i} u_{1} \sigma_{1} / \bar{v} \sigma_{e s}\right]\right)
$$

Here $b$ is the electron trajectory due to elastic and inelastic collisions, given by equation (22), and $\bar{v}$ is a characteristic velocity of the resulting ionization or excitation rate, $\mathrm{dn}_{\hat{j}}^{\star}$ in the $\mathrm{PL}$, given by

$$
\bar{v}=(1 / 4) q_{e s}\left(\varepsilon_{e s}\right) a_{q}(P, E) \gamma\left(\varepsilon_{i s}, T_{s}\right) u_{q}\left(T_{g}, P, E\right)
$$

with of and oes the cross sections for ionization and electron-neutral collisions, respectively, $T_{s}$ and $T_{g}$ the substrate and plasma temperatures, respectively, $P$ the total gas pressure and $E$ the electric field strength in the sheath.

In the following discussion of closed (nonflowing gases) and open (flowing gases) systems the PL is considered to govern both the polymerization (hydrocarbons, chlorosilanes, etc.) and deposition rates.

Deposition Rate in Closed Plasma System. - These systems have been discussed by Poll et al. (1976). Nonflowing gases are characterized by $v_{f}=0$ or $Q=0$. Following khait et al. (1980) we consider $\Delta L$, the length of $P L(f \mathrm{~g} .13)$. Let $\Delta t_{R}$ be the time interval in which $n_{0}$ (the quantity of monomer material in the gas mixture to be polymerized, i.e., $\left.n_{0}=n_{M} / n_{m i x}\right)$ at $t=0$ is transformed after polymerization to a final ' $n_{f}$, and $S_{0}$, the $P L$ cross section, is transformed into $S_{0}^{\prime}$, the cross section of the substrate where deposition occurs (after the time interval, $\left.\Delta t_{R}\right)$. At any time $t$, the fraction $\delta n_{m}(t)$ of material particles deposited can be expressed as:

$$
\delta n_{m}(t)=\left(1 / m_{0}\right) \int_{0}^{t}(d G / d t) d t
$$


where $m_{0}$ is the mass material deposited on the substrate. If we assume that only a fraction $\alpha<1$ of monomer is excited, ionized and polymerized by $\bar{\varepsilon}_{e s}$, then the rate of deposition $\mathrm{dG} / \mathrm{dt}$ is:

$$
(d G / d t)_{v_{f}=0}=m_{0} \alpha\left(d n_{m}^{\star}(+) / d t\right)
$$

By applying equations (68) and (69) for PL we get:

$$
(d G / d t)_{v_{f}=0}=(1 / 4) m_{0} \alpha q_{e s} \gamma a_{1} n u_{1} S_{0}^{\prime}
$$

According to Poll et al. (1976) at $v_{f}=0$ or $Q=0$ the relative concentration of monomer decreases with time according to:

$$
\eta_{f}(t)=n_{0} \exp \left(-t / \Delta t_{R}\right)
$$

The time interval for deposition, $\Delta t_{R}$, is given by

$$
\Delta t_{R}=\Delta L / \nabla \alpha
$$

where $\bar{v}$ is the characteristic velocity of the resulting ionization, excitation, and/or polymerization. Thus,

$$
(d G / d t)_{v_{f}=0}=\left[n_{m}(0) / \Delta t_{R}\right] m_{0} \exp \left(-t / \Delta t_{R}\right)
$$

It follows that the rate of deposition in a plasma system with nonflowing gases decreases exponentially with the duration of operation. As the data in table XV indicates this result can be applied to the consumption rate of the monomer, $(d M / d t) v_{f}=0$. Koizlik (1974), Poll et al: (1976) and Luhleich et a). (1977) have confirmed experimentaliy the exponential decay of $\mathrm{dG} / \mathrm{dt}$ in a plasma with nonflowing gases (closed system).

Deposition Rate in Open Plasma Systems. - With $v_{f}>0, \Delta t_{R}$ is the time interval in which $n_{0} \rightarrow n_{f}, S_{0} \rightarrow S_{0}^{1}$ and the flow velocity $v_{f} \rightarrow v_{f}^{\prime}$. At steady state:

$$
\left(i / m_{0}\right) d G / d t+\left[n_{m}(0)-n_{m}\right] / \Delta t_{R}=0
$$

and the rate of deposition is:

$$
d G / d t=\left[m_{0} n_{m} / \Delta t_{R}\right] \beta_{\psi}(v)
$$

where $B=S_{0} / S_{0}^{\prime}$ is a geometric factor and $\psi(v)$ an efficiency coefficient of excitation, ionization and polymerization of particles having flow velocity $v_{f}^{\prime}$.

In the PL with HEEB interaction:

$$
\Delta t_{R} / \beta=\Delta L / \beta \alpha \bar{v}
$$

where $\bar{v}$ is again the characteristic velocity of the resulting $\mathrm{dn}_{i}^{\star}$ or polymerization in the PL. The coefficient $\psi(v)$ behaves such that at velocity $v_{f}=0, \psi(v)=1$, representing the maximum ionization, 
excitation or polymerization and deposition rate. As $v_{f} \rightarrow \infty, \psi(v) \rightarrow 0$ i.e.,

$$
\Psi(v / v)=1-\exp \left(-\bar{v} \alpha \beta / v_{f}\right)
$$

Under these conditions the rate of deposition is given by

$$
d G / d t=m_{0} n_{f}\left(d n_{i}^{\star} / d t\right)_{v_{f}}, \Delta V V \bar{v}_{e f f}
$$

where

$\Delta V \quad$ plasma layer volume

$\bar{v}_{\text {eff }}$ in dimensions of velocity includes the coefficients $\alpha, \beta$, and $\psi(v / \bar{v})$ :

$$
\bar{v}_{\text {eff }}=\alpha \beta \psi(v / \bar{v}) v_{f} \bar{v} /[\alpha \bar{v} \psi(v / \bar{v}) \beta]+v_{f}
$$

Equation (81) cannot be solved analytically. It can be numerically evaluated as follows:

The experimental efficiency of deposition is the ratio between the deposition rate and the rate of monomer consumption, that is,

$$
[(d G / d t) /(d M / d t)]_{P L}=\bar{v}_{e f f} / v_{f}
$$

Table XV gives the ratios of $-\bar{v}_{e f f} / v_{f}$ as evaluated from experimental values of $\mathrm{dG} / \mathrm{dt}$ and monomer consumption, $\mathrm{dM} / \mathrm{dt}$. For the various $\mathrm{rf}$. plasmas listed in table XV the ratio $\bar{v}_{e f f} / v_{f}$ varies between 0.34 for a boridation process to 0.48 for the pyrocarbon deposition from $\mathrm{C}_{3} \mathrm{H}_{6}+\mathrm{Ar}$ plasma. Depending on the flow velocity, $v_{f}$, the effective velocity, $\bar{v}_{e f f}$, can be selected for optimizing the rates of deposition and monomer consumption. Figures 59 to 61 show the experimental values of $\mathrm{dG} / \mathrm{dt}$ and the calculated values for $\bar{v}_{e f f} / v_{f}=0.46$ in the pyrocarbon deposition from mixtures of propylene and argon in an rf plasma. The agreement between the predicted and measured values suggested the validity of the theoretical model of Khait et al. (1980) for flow systems.

\section{CONCLUDING REMARKS}

The understanding of $\mathrm{film}$ deposition processes in plasma systems is still in its infancy. The deposition "models" described in the preceding section have a phenomenologically qualitative character. There is insufficient information on plasma surface interactions (PSI) chiefly due to the fact that few experiments have been done with known fluxes of radicals on well-defined surfaces and little is known about synergetic effects in plasmas. To remedy this situation basic research in the following areas is needed:

(1) Recombination reactions at a surface where radicals recombine to form stable molecules.

(11) Synergetic effects where photon, electron and fon bombardment change the reactivity of the incident radical with the surface. 
(11i) Adsorption characteristics of the incident species as a function of surface coverage and plasma conditions, such as electrical variables, bias voltages on the substrate, and partial pressure.

(iv) Measurements of the rate of conversion of reactants to products on a surface.

(v) The growth mechanism in the case of polymer deposition.

It would be most desirable to perform well-defined surface experiments inside a plasma system. At present this seems to be very difficult if not impossible. A step in the right direction would be to apply both in-situ and off-line techniques. For example, an insight to the PSI can be obtained by in-situ measurements of the plasma layer (table XI). Optical spectroscopy such as emission, adsorption, laser-induced fluorescence, laser optogalvanic effect and laser induced Raman effect can analyze the particles' flux gradients to the surface and monitor those sputtered away and/or etched from the surface. The information gained would be the reaction rates of excitation, ionization, dissociation, formation of polymeric species and clusters. Off-line measurements of the deposited films by techniques such as Auger, LEED, UPS, XPS, SIMS infrared and Raman spectroscopy, and EPR will supply data on surface coverage, sticking coefficients, nucleation, chemical bondings, and rates of diffusion and segregation. The correlation between the "in-situ" and the "off-line" measurements could form the basis for developing appropriate theoretical models on the film deposition processes in glow discharges. 


\section{REFERENCES}

d'Agostino, R. (1983). Proc. Sixteenth Intern. Conf. Phenom. Ionized Gases, Duesseldorf.

d'Agostino, R., Cramarossa, F., De Benedictis, S., and Ferraro, G. (1981a). J. App 1. Phys. $\underline{52}(3), 1259$.

d'Agostino, R., Colaprico, V., and Cramarossa, F. (1981b). Plasma Chem. Plasma Process. I, 365.

d'Agostino, R. Cramarossa, F., and de Benedictis, S. (1982). Plasma Chem. Plasma Process. 2., 213.

d'Agostino, R., Cramarossa, F., Colaprico, V., and s'Ettole, R., (1983a). J. App 1. Phys. $\underline{54}, 1284$.

d'Agostino, R., Cramarossa, F., and de Benedictis, S. (1983b). Proc. Sixth Intern. Symp. Plasma Chem., Montreal, p. 394.

d'Agostino, R., de Benedictis, S., and Cramarossa, F. (1984C). Plasma Chem. Plasma Process. 4 , (in press).

A7t, L. L., Ing, Jr., S. W., and Laendle, K. W. (1963). J. Electrochem. Soc., $\underline{110}, 465$.

Anderson, D. A., and Spear, W: E.. (1977). Phil. Mag. 35, 1.

Anderson, G. S., Mayer, Wm. N., and Wehner, G. K. (1966). J. Appl. Phys. 33, 2991 .

Ando, K., and Matsumura, K. (1978). Thin Solid Films, 52, 153.

Akash1, K., Taniguch1, Y., and Takada, R. (1981). Proc. Fifth Intern. Symp. Plasma Chem., Edinburgh, p. 376.

Arnold, H., Biste, L., Bolze, D., and Elchhorn, G. (1976). Krist. Tech. 11, 17.

Avn1, R. (1984). Invited Lecture at 11 th Intern. Conf. Meta11. Coatings, San Diego, April 1984.

Avn1, R., Carmi, U., Inspektor, A., and Rosenthal, R (1984). NASA Tech. Paper 2301, Apri1 1984 .

Avn1, R., Carmi, U., Manory, R., Grossman, E., and Gri11, A. (1983a). Proc. Sixth Intern Symp. Plasma Chem., Montreal, p. 820.

. Avn1, R., Carmi, U., Rosentha 1, I., and Inspektor, A. (1983b). Proc. Sixth Intern. Symp. Plasma Chem., Montreal, p. 522.

Avni, R., Carmi, U., Rosenthal, I., Manory, R., and Gri11, A. (1983C). Thin Solid Films, 107, 235.

Ba11, D. J., (1972). J. Appl. Phys. $\underline{43}, 3047$. 
Bass, A. M., and Broida, H. P. (eds.) (1960). "Formation and Trapping of Free Radicals", Academic Press, New York.

Bauer, E., and Poppa, H. (1972). Thin Solid F1lms, 12, 167.

Bauer, G. H., and Bilger, G. (1981). Thin Soltd Films, 83, 223.

Bauer, J., Biste, L., and Bolze, D. (1977). Phys. Status Solid1, A $\underline{39}, 173$.

Baum, T. (1927). Z. Physik, 40, 686.

Be11. A. T. (1980). In "Plasma Chemistry III" (S. Veprek and M. Venugopalan, eds.). Springer Verlag, Berlin, pp. 43-68.

Be 11. A. T. (1976). In "Plasma Chemistry of Polymers" (M. Shen, ed.) Marcel Dekker, New York, pp. 1-13.

Berghouse, B. (1932). German Patent 669,639, July 1932.

Bergstedt, K., Ferguson, E., Schluter, and Wulff, H. (1962). Proc. Fifth Intern. Conf. Ion. Phenom. Gases, Munich, Vol. 1, p. 437 . North-Holland, Amsterdam.

Bethe, H. A., and Salpeter, E. E. (1957). "Quantum Mechanics of One and Two Electron Atoms". Springer Verlag, Berlin.

Boehme, D. K., and Goodings, J: M. (1966). Rev. S1. Instr. $\underline{37}, 362$.

Boenig. H. V. (1982). "Plasma Sclence and Technology" Cornell Untv. Press, Ithaca, New York.

Boer, J. H. D. (1969). In "Molecular Processes on Solid Surfaces" (E.

Dragulis, R. D. Gretz and R. I. Jaffe, eds.) McGraw-Hill, New York.

Bogen, P. and Hintz, E. (1978). Comments on Plasma Physics, Contr. Fusion, 4 , 115 .

Bohm, D. (1949). In "The Characteristics of Electrical Discharges in Magnetic Fields" (A. Guthrie and R. K. Wakerling, eds.) McGraw-Hill, New York.

Bondybey, V. E., and Miller, T. A. (1977). J. Chem. Phys. 66, 3337.

Bondybey, V. E., and Miller, T. A. (1978). J. Chem. Phys. 69, 3597.

Bondybey, V. E., and Mtller, T. A. (1979). J. Chem. Phys. 70, 138.

Bonifield, T. D. (1982). In "Deposition Technologies for Films and Coatings" (R. F. Bunshah, ed.) Noyes Publ., Park Ridge, N. J., pp. 365-384.

Bosse, J. V., Richter, K., and Kruppa, E. F. (1932). Swiss Patent 172,436, July 1932.

Bradley, A., and Hammes, J. P. (1963). J. Electrochem. Soc. 110, 15.

Braganza, C., Veprek, S., Wirz, E., Stuessi, H., and Textor, M. (1979). Proc. Fourth Intern. Symp. Plasma Chem., Zurich, p. 100. 
Brainard, W. A., and Wheeler, D. R. (1978). J. Vac. Sci. Technol. 15, 1800. Breckenridge, W. H., and Miller, T. A. (1972). J. Chem. Phys. 56, 475. Brokman, A., and Tuler, F. R. (1987). J. App1. Phys. 52, 468.

Brown, J. (1972). MTP Int. Rev. Sc1. 4, 235.

Brueck, S. R. J., and Ehrlich, D. J. (1982). Phys. Rev. Lett. $\underline{48}, 1678$. Brueck, S. R..J., and Pang, S. (1983). Proc. Sixth Intern. Symp. Plasma Chem., Montrea 1, p. 714.

Bruno, G., Capezzuto, P., Colaprico, V., d'Agostino, R., and Cramarossa, F. (1979). Proc. Fourth Intern. Symp. Plasma Chem., Zurich, p. 433.

Bruno, G., Capezzuto, P., Cramarossa, F., and d'Agostino, R. (1980). Thin Solid Films, 67, 103.

Bruno, G., Capezzuto, P., Cramarossa, F., Ange111, V., Murr1, R., Schiavul11, L., Fortunato, G., and Evangelisti, F. (1981). Proc. Fifth Intern. Symp.

Plasma Chem., Edinburgh, p. 652.

Burakov, V. S., Misyakov, P. Y., Naumenko, P. A., Nechaev, S. V., Razdobarin, G. T., Semenov, V. V., Sokolova, L. V., and Folomkin, I. P. (1977). JETP Lett., 26. 403 .

Burakov, V. S. (1978). J. App1. SpectrosC. (USSR) 29, 1504.

Butler, H. S., and Kino, G. S. (1963). Phys. Fluids, $\underline{6}, 1346$.

Cabannes, F. and Chapelle, J. (1971). In "Reactions Under Plasma Conditions" (M. Venugopalan, ed.) Vol. I., pp. 367-469. Wiley, New York.

Capite111, M. and Molinari, E. (1980). In "Plasma Chemistry II" (S. Veprek and M. Venugopalan, eds.), Springer Verlag, Berlin, p. 59.

Carmi, U., Inspektor, A., and Avni, R. (1981). Plasma Chem. Plasma Process. 1. 233 .

Carrington, A., Levy, D. H., and Miller, T. A. (1970). Adv. Chem. Phys. 18, 149 .

Catherine, Y., and Turban, G. (1977). Intern. Round Table Surf. Treat. Plasma Polymer, IUPAC, Limoges, Fr.

Catherine, Y., and Turban, G. (1979). Thin Solid F11ms, 60, 193.

Chang, R. P. H. (1979). Thin Solid Films, 56, 89.

Chang, R. P. H., Chang, C.C., and Darack, S. (1980). Appl. Phys. Lett. $\underline{3}$, 999.

Chapman, B. (1980). "Glow Discharge Processes". Wliey, New York.

Chapman, B. N., Downer, D., and Guimaraes, L. J. M. (1974). J. App 1. Phys. 45. 2115 . 
Chatham, H., Hils, D., Robertson, R., and Gallagher, A. C. (1983). J. Chem. Phys. $19,1301$.

Chen, F. F. (1965). In "Plasma Diagnostic Techniques". (R. H. Huddlestone and S. L. Leonard, eds.) pp. 113-200.

Cherrington, B. E., (1982) Plasma Chem. Plasma Process. 2, 113.

Chung, P. M., Talbot, L., and Touryan, K. J. (1975). "Electric Probes in Stationary and Flowing Plasmas: Theory and Application", Springer Verlag, Berlin.

Clements, R. M. (1978), J. Vac. Sc1. Technol. 15(2), 193.

Cobine, J. D. (1958). "Gaseous Conductors". Dover, New York.

Coburn, J. W. (1970). Rev. Sci. Instrum. 41, 1219.

Coburn, J. W: (1982). Plasma Chem. Plasma Process. 2, 1.

Coburn, J. W., and Chen, M. (1980). J. Appl. Phys. 51(6), 3134.

Coburn, J. W., and Kay, E. (1971). Solid State Technol. Dec. 1971, p. 49.

Coburn, J. W., and Kay, E. (1972). J. App1. Phys. 43, 4965.

Coburn, J. W., Taglauer, E., and Kay, E. (1974) Japan J. App1. Phys. Supp1. 2, Pt. 1, p. 501 .

Cook, J. M., Miller, T. A., and Bondybey, V. E. (1978) J. Chem. Phys. 69, 2562.

Content, M., Michel, H., and Gantois, M. (1974), Trait. Therm. 88, 57.

Cramarossa, F., d'Agostino, R., and de Benedictis, S. (1983). Proc. Fourth Intern. Conf. Ion Plasma Assisted Techniques, Kyoto.

Cuomo, J. J., Gambino, R. J., Harper, J. M. E:, Kutsis, J. D., and Webbe, J. C., (1978), J. Vac. Sc1. Technol. 15, 281.

Davis, W. D., and Vandersilce, T. A. (1963). Phys. Rev. 137, 219.

Dell'oca, C. J., Pulfrey, D. L., and Young, L. (1971). In "Physics of Thin Fllms" (M. H. Francombe and R. W. Hoffmann, eds.), Vol. 6. Academic Press, New York.

Denaro, A. R., Owens, P. A. and Crawshaw, A. (1968). European Polymer J., 4 , 93.

Denaro, A. R., Owens, P. A. and Crawshaw, A. (1969). European Polymer J., $\underline{5}$, 471 .

Denaro, A. R., Owens, P. A. and Crawshaw, A. (1970), European Polymer J., $\underline{6}$, 487.

Donnelly, V. M., and Flamm, D. L. (1983). See Ibbotson et al. (1983). 
Drawin, H. W. (1971). In "Reactions Under Plasma Conditions" (M. Venugopalan, ed.). Vol. I, pp. 53-238. Wlley, New York.

Drevilion, B., Huc, J., Lloret, A., Perrin, J., de Rosny, G., and Schmitt, J. P. M. (1980) App 1. Phys. Lett. 37, 646.

Dun, H., Pan, P., White, F. R., and Douse, R. W. (1981) J. Electrochem. Soc. 128,1555 .

Eckbreth, A. C., Bonczyk, P. A., and Verdleck, J. F. (1977). Appl. Spectrosc. Rev. 13. 15

Eckbreth, A. C., Bonczyk, P. A., and Verdleck, J. F. (1979) Prog. Energy Combust. Sci. $4,253$.

Edenhofer, B. (1974). Heat Treat. Met. I, 23.

Ehrlich, D. J. and Tsao, J. Y. (1983), J. Vac. Sc1. Technol. 18, 969.

Ehrlich, D. J., Osgood, R. M., and Deutsch, T. F. (1981), Appl. Phys. Lett. 38, 946 .

Elbern A., (1978). App1. Phys. 15, 111.

Elbern, A. Rusbueldt, D., and Hintz, E. (1977). Proc. Intern. Symp.

Plasma-Wall Interactions, p. 475. Pergamon Press, Oxford.

Elbern, A., Hintz, E., and Schweer, B. (1978). J. Nuc1. Mat. 76-77 (1-2), 143.

Enge 1, A. V. (1965). "Ionlzed Gases". Oxford Univ. Press, Oxford.

Engemann, D., Fischer, R., and Knecht, J. (1978). Appl. Phys. Lett. $32,567$.

Erskine, J. C., and Cserhati, A. (1978). J. Vac. Sc1. Technol. 15, 1823.

Eser, E., Ogilvie, R. E., and Taylor, K. A. (1978). J. Vac. Scl. Technol. $\underline{15}(2), 199$.

Eubank, A., and Sindoni, E. (eds.) (1975) "Courses on Plasma Diagnostics and Data Acquistion Systems". Editrice Compositori, Bologna.

Flamm, D. L., and Donnelly, V. M. (1981), Plasma Chem. Plasma Process. 1, 317.

Foner, S. N., and Hudson, R. L. (1953). J. Chem. Phys. 21, 1374.

Foner, S. N., and Hudson, R. L. (1962). Advan. Chem. Ser. $\underline{36}, 34$.

Franklin, J. L., Studntarz, S. A., and Ghosh, P. K. (1968). J. Appl. Phys. 39. 2052.

Fraser, D. B. (1978). In "Thin Film Processes" (J. L. Vossen and W. Kern, eds.). Academic Press, New York, pp. 115-129.

Fromhold, Jr., A. T. (1977). J. Electrochem. Soc. 124, 538.

Fromhold, Jr., A. T. (1982) Thin Soltd Films, 95, 297. 
Fromhold, Jr., A. T., and Baker, M. (1980). J. Appl. Phys. 51, 6377.

Gafri, 0., Gri11, A., Itzhak, D., Inspektor, A., and Avni, R. (1980). Thin Solid Films, $\underline{72}, 523$.

Gazlck1, M., and Yasuda, H. (1983) Plasma Chem. Plasma Process. $\underline{3}, 279$.

Gereth, R., and Scherber, W. (1972) J. Electrochem. Soc., 119. 1248.

Gieres, G. (1983) Proc. S1xth Intern. Symp. Plasma Chem., Montreal, p. 456.

Glockler, G., and Lind, S. C. (1939), "The Electrochemistry of Gases and Other Dielectrics". Wiley, New York, pp. 400-429.

Gourrier, S., and Bacal, M. (1981). Plasma Chem. Plasma Process. 1, 217.

Gourrier, S., Mircea, A., and Bacal, M. (1980). Thin Solid F1lms, $\underline{65}, 315$.

Greene, J. E. (1978). J. Vac. Sci. Technol. 15(5), 1718.

Greene, J. E., and Sequeda-Osorio, F. (1973). J. Vac. Sci. Technol. 10, 1144.

Greene, J. E., Sequeda-0sorio, F., and Natarajan, B. R. (1975), J. Appl. Phys. 46. 2701 .

Greene J. E., Natarajan, B. R., and Sequeda-Osorio, F. (1978), J. Appl. Phys., 49. 417.

Greiner, J. H. (1971). J. App1. Phys. 42, 5151.

Greiner, J. H. (1974). J. App 1. Phys. $45,32$.

Grenda, M. S. and Venugopalan, M. (1980), J. Polym. Sc1. Poly. Chem. Ed., 18. 1611 .

Griem, H. R. (1964). "Plasma Spectroscopy", McGraw-H111, New York.

Griffith, R. W., Kampas, F. J., Vanier, P. E., and Hirsch, M. D. (1980), J. Non-Cryst. Solids, $\underline{35} / \underline{36}, 391$.

Gri11, A., Grossman, E., Manory, R., Carmi, U., and Avni, R. (1983). Proc. Sixth Intern. Symp. Plasma Chem., Montrea1, p: 843.

Grimberg, A., Avnt, R., and Gril1, A. (1982) Thin Soltd Films, 96, 163.

Groh, K. H., and Reuschling, R. (1981). Proc. Fifth Intern. Symp. Plasma Chem. Edinburgh, p. 493.

Grossman, E., Avn1, R., and Gr111, A. (1981) Proc. Fifth Intern. Symp. Plasma Chem.. Edinburgh, p. 658.

Grossman, E., Avni, R., and Gri11, A. (1982a)! Thin Solid Films, 90, 237. 
Grossman, E., Gri11, A., and Avn1, R., (1982b). Plasma Chem. Plasma Process., 2. 341 .

Grove, W. R. (1852), Ph11. Trans. Roy. Soc. London, 142, 87.

Grube, W. L., and Gay, J. G. (1978). Meta11. Trans. 9A, 1421.

Gruen, D. M., Gaudioso, S. L., McBeth, R. L., and Lerner, J. L. (1974). J.

Chem. Phys. 60, 89.

Gruen, D. M., Veprek, S., and Wright, R. B. (1980). In "Plasma Chemistry I"

(S. Veprek and M. Venugopalan, eds.). Springer Verlag, Berlin, pp. 96-99.

Gruen, D. M., Krauss, A. R., Susman, S., Venugopalan, M., Ron, M. (1983). J. Vac. Sc1. Technol. Al(2), 924.

Guentherschulze, A. (1926), Z. Physik $\underline{36}, 563$.

Hagstrum, H. D. (1961). Phys. Rev. 123, 758.

Hagstrum, H. D. (1977). In "Inelastic Ion-Surface Collisions" (N. H. Tolk, J. C. Tully, W. Helland and C. W. White, eds.) Academic Press, New York.

Haller, I. (1980). Appl. Phys. Lett. $\underline{37}, 282$.

Hanak, J. J. and Peallicane, J. P. (1976), J. Vac. Sc1. Technol. 13, 406.

Haque, M. R., Oswald, H. R., and Veprek, S. (1975) Proc. Twelfth Intern. Conf. Phenom. Ionized Gases, Pt. I, Eindhoven, p. 42.

Harper, J. M. E., Heiblum, M., Speidell, I. L., and Cuomo, J. J. (1981). J. App 1. Phys. $\underline{52}, 4118$.

Harshbarger, W. R., Młller, T. A., Norton, P. and Porter, R. A. (1977). Appl. Spectrosc. 31. 201.

Harshbarger, W. R., Porter, R. A., and Norton, P. (1978). J. Electron Mater. I. 429 .

Hasted, J. B. (1975). Intern. J. Mass Spectrom. Ion Phys. 16, 3.

Hata, N., Matsuda, A., Tanaka, K., Kajfyama, K., Moro, N., and Sajfki, K. (1983a) Japan J. Appl. Phys. 22, L1.

Hata, N., Matsuda, A., and Tanaka, K. (1983b). Proc. Tenth Intern. Conf. Amorphous and Liquid Semiconductors, Tokyo.

Hata, N., Matsuda, A., and Tanaka, K. (1983C). Proc. Fourth Intern. Conf. Ion and Plasma Assisted Techniques, Kyoto.

Hauser, J. J. (1977). J. Non-Cryst. Solids, 23, 21.

Havens, M. R., Biols1, M. E., and Mayhan, K. G. (1976) J. Vac. Sci. Technol. $\underline{13}, 575$. 
Hayashi, T., Mlyamura, M., and Komiya, S. (1982) Japan J. App1. Phys. 21, L755. Hayashi; T., Kikuchi, M., Fujioka, T., and Komiya, S. (1983). Proc. Intern. Ion Eng. Congr.-ISIAT IPAT, Kyoto, Sep. 1983, p. 1611.

Hazelwood, F. J. (1978) Intern. Conf. Adv. Surf. Coat. Technol., London, p. 29. He Im, H. (1974). J. Phys. B7, 170.

Hippel, A. V. (1926). Ann Physik, 80, 672.

Ho, V. Q.. and Sugano, T. (1979). Proc. Third Intern. Conf. Solid State Devices, Tokyo.

Ho, V. Q., and Sugano, T. (1982). Thin Solid F1lms, 95, 315.

Hollahan, J. R. (1979) J. Electrochem. Soc. 126, 930.

Hollahan, J. R., and Rosler, R. S. (1978) In "Thin F1lm Processes" (J. L. Vossen and W. Kern, eds.) Academic Press, New York, p. 354.

Holland, L. (1974), In "Science and Technology of Surface Coating" (B. N. Chapman and J. C. Anderson, eds.) Academic Press, New York.

Holland, L., and OJha, S. M. (1976a). Thin Solid Films, 38, L17.

Holland, L., and 0jha, S. M. (1976b). Vacuum, 26, 53.

Hontg, R. E. (1958). J. Appl. Phys. 29, 549.

Horsley, A. W. (1969), Electronics, $\underline{42}, 84$.

Hosokawa, N., Konishi, A., Hiratsuka, H., and Annoh, K. (1980). Thin Solid F11ms, 73, 115 .

Huddlestone, R. H., and Leonard, S. L. (eds.) (1965). "Plasma Dlagnostic Techniques". Academic Press, New York.

Hudis, M. (1973). J. Appl. Phys. 44, 1489 .

Hyder, S. B., and Yep, T. O. (1976) J. Electrochem. Soc. 123, 1721.

Ibbotson, D. E., Flamm, D. L., and Donnelly, V. M. (1983), J. Appl. Phys. 54. 5974 .

Ing, S. W., and Davern, W. (1964) J. Electrochem. Soc. 111, 120.

Inspektor, A., Carmi, U., Avni, R., and Nicke1, H. (1979) Proc. Fourth Intern. Symp. Plasma Chem., Zurich, p. 390.

Inspektor, A., Carmi, U., Avni, R., and Nickel, H. (1981), Plasma Chem. Plasma Process. 1. 377.

Joyce, R. J., Sterling H. F., and Alexander, J. H. (1968) Thin Solid Films, I, 481 .

Kaminsky, M. (1965). "Atomic and Ionic Impact Phenomena on Metal Surfaces". Springer Verlag, Berlin. 


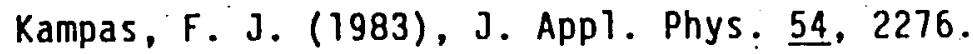

Kampas, F. J., and Griffith, R. W. (1980) Solar Cells, 2, 385.

Kampas, F. J., and Griffith, R. W. (1981a). Appl. Phys. Lett. 39, 407.

Kampas, F, J., and Griffith, R. W. (1981b). J. App1.Phys. 52. 1285.

Katayama, D. H., Młller, T. A., and Bondybey, V. E. (1980), J. Chem. Phys. 72. 5469 .

Katto, H., and Koga, Y. (1971) J. Electrochem. Soc. 118, 1619.

Katz, M., Gri11, A., Itzhak, D., and Avn1, R. (1979). Proc. Fourth Intern. Symp. Plasma Chem., Zurich, p. 444.

Katz, M., Itzhak, D., Gri11, A., and Avn1, R. (1980). Thin Solld F1lms, 72 , 497.

Kaufman, F. (1969). Adv. Chem. Ser. $\underline{80}, 29$.

Kaufman, M. (1976). Pure Appl. Chem. 48, 155.

Kay, E. (1977) Intern. Round Table Plasma Polymer. Treat., IUPAC Symp. Plasma Chem. Limoges, Fr.

Kay, E., and Dilks, A. (1979) ACS Symp. Ser. 108, 195.

Kay, E., and Dilks, A. (1981) J. Vac. Sci. Technol. 18, 1.

Kay, E., and Hecq,.M. (1983) Proc. Sixth Intern. Symp. Plasma Chem., Montreal, p. 490 .

Kay, E., Coburn, J. W., and Kruppa, G. (1976). Vide 183, 89.

Kay, E., D11ks, A., and Hetzler, U. (1978) Macromol. Sc1.-Chem. A12(9), 1393.

Kay, E., Coburn, J., and Dilks, A. (1980). In "Plasma Chemistry III", (S.

Veprek and M. Venugopalan, eds.), Springer Verlag, Berlin, pp. 1-42.

Kern, W., and Rosler, R. S. (1977). J. Vac. Sc1. Technol. 14, 1082.

Khatt, Y. L., Inspektor, A., and Avni, R. (1980), Thin Solid Films, 72, 249.

Khatt, Y. L., Carmi, U., and Avni, R. (1983). Proc. Sixth Intern. Symp.

Plasma Chem., Montreal, p. 729.

Kimmitt, M. F., Prior, A. C., Roberts, V. (1965). In "Plasma Diagnostic Techniques" (R. H. Huddlestone and S. L. Leonard, Eds.). Ch. 9, pp. 399-430. Academic Press, New York.

Kirk, R. (1974) In "Techniques and Applications of Plasma Chemistry" (J. R. Hollahan and A. T. Bell, eds.), Ch. 9, Wlley, New York.

Klinger, R. E., and Greene, J. E. (1981). Appl. Phys. Lett. $\underline{38}(8), 620$. 
Knights, J. C., (1980), J. Non-Cryst. Solids, $\underline{35} / \underline{36}, 159$.

Knights, J. C., Lujan, R. A., Rosenblum, M. P., Street, R. A., Biegelsen, D. K., and Reimer, J. A. (1981), Appl. Phys. Lett. $\underline{38}, 331$.

Knights, J. C., Schmitt, J. P. M., Perrin J., and Guelachvi11, G. (1982). J. Chem. Phys. $\underline{76}(7), 3414$.

Koenig, H. R., and Maisse1, L. I. (1970), IBM J. Res. Dev., 14, 168.

Koizlik, K. (1974). KFA Report 1128RW, Kernforschungsanlage, Juelich.

Kolotyrkin, V. M., Gllman, A. B., and Tsapuk, A. K. (1967). Russ. Chem. Rev. 36. 579 .

Komiya, S., Yoshikawa, K., and Ono, S. (1977). J. Vac. Sc1. Techno1. 14, 1161.

Konuma, M., and Matsumoto, 0. (1977), J. Less-Common Meta 1s, 52, 145; $\underline{55}, 97$.

Konuma, M., Kanzaki, Y., and Matsumoto, 0. (1979). Proc. Fourth Intern. Symp. Plasma Chem., Zurich, p. 174.

Kueppers, D. and Lydtin, H. (1980) In "Plasma Chemistry I" (S. Veprek and $M$. Venugopalan, eds.), Springer Verlag, Berlin.

Kueppers, D., Koenings, J., and Wilson, H. (1976) J. Electrochem. Soc. 123, 1079.

Kueppers, D., Koenings, J., and wilson, H. (1978) J. Electrochem. Soc. 125, 1298.

Kumar, V. (1976) J. Electrochem. Soc. 123, 262.

Kuwano, Y. (1968) Japan J. App 1. Phys. I, 88.

Kuwano, Y. (1969) Japan J. App1. Phys. ․, 876:

Laegried, N., and Wehner, K. G. (1961). J. App1. Phys. 32, 365.

Lakhtin, Yu. M., Kogan, Ya. D., and Saposhnikov, V. N. (1976). Metalloved. Term. Obrab. Met. $\underline{6}$, 2(1976).

Lam, D. K., Baddour, R: F. and Stance11, A. F. (1976). In "Plasma Chemistry of Polymers". (M. Shen, ed.). Marcel Dekker, New York.

Langmuir, I., and Mott-Smith, H. M. (1924). Gen. Elec. Rev. 27, 449.

Lebrun, J. P., Miche1, H., and Gantois, M. (1972), Mem. Sc1. Rev. Metal1. 69, 727.

LeComber, P. G., and Spear, W. E. (1979). In "Amorphous Semiconductors". (M. H. Brodsky, ed.). Springer Verlag, Berlin, pp. 251-284.

LeContellec, M., Richard, J., Guivarc'h, A., Ligeon, E., and Fontenille, J. (1979) Thin Solid Films $\underline{58}, 407$.

Lergon, H. G., and Mueller, K. G. (1977). Z. Naturforsch. 32A, 1093. 
Lergon, H. G., Venugopalan, M., and Mueller, K. G. (1984) Plasma Chem. Plasma Process. 4, 107.

Leslie, J. D., Keeth, V., and Knorr, K. (1978). J. Electrochem. Soc, 125, 44.

Lewicki, G., and Mead, C. A. (1971) Phys. Rev. Lett. 16, 266.

Ligenza, L. R. (1965). J. Appl. Phys. 36, 2703.

Liu, M. B., Gryen, D. M., Krauss, A. R., Rets, Jr., A. H., and Peterson, S. W. (1978) High Temp. Sc1. 10, 53.

Loechte-Holtgreven, W. (ed.) (1968). "Plasma Diagnostics", North-Holland, Amsterdam.

Loechte-Holtgreven, W., and Richter, J. (1968). In "Plasma Diagnostics" (W. Loechte-Holtgreven, ed.) Ch. 5 pp. 250-346. North-Holland, Amsterdam.

Lothe, J., and Pound, G. M. (1969). In "Molecular Processes on Solid Surfaces" (E. Dragulis, R. D. Gretz and R. I. Jaffe, eds.) McGraw-H111, New York.

Luhleich, H., Seeberger, D., Suetteriin, L., and von Seggern, R. (1977). High Temp. High Press. 9 , 283.

Manory, R., Grill, A., Carmi, U., and Avni, R. (1983). Plasma Chem. Plasma Process. $\underline{3}, 235$.

Marcyk, G. T., and Streetman, B. G. (1977), J. Vac. Sc1. Technol. 14, 1165. Mashita, M., and Matsushima, K. (1974) Japan J. App 1. Phys. Supp1. 2, Pt. 1, p. 761 .

Matsuda, A., and Tanaka, K. (1982). Thin Solidd Films, 92, 171.

Matsuda, A., Nakagawa, K., Tanaka, K., Matsumura, M., Yamasak1, S., Okush1, H., and Itjima, S., (1980). J. Non-Cryst. Soltds, $\underline{35} / \underline{36}, 183$.

Matsuda, A., Kaga, T., Tanaka, H., and Tanaka, K. (1983a), Proc. Tenth Intern. Conf. Amorphous and Liquid Semiconductors, Tokyo.

Matsuda, A., Kaga, T., Tanaka, H., Malhotra., $L_{i}$, and Tanaka, K. (1983b), Japan J. Appl. Phys. 22, L115.

Matsuda, A., Kumaga 1, K., and Tanaka, K. (1983c). Japan J. App1. Phys. 22, L34.

Matsumoto, 0., and Yatsuda, Y. (1981) Proc. Fifth Intern. Symp. Plasma Chem., Edinburgh, p. 382 .

Matsumoto, 0., and Kanazaki, Y. (1983). Proc. Sixth Intern. Symp. Plasma Chem., Montrea1, p. 340.

Matsumoto, 0., Konuma, M., and Kanzak, Y. (1982). J. Less-Common Meta 1s, 84, 157. 
Mattox, D. M. (1964), Electrochem. Technol. 2., 295.

Mattox, D. M. (1973), J. Vac. Sci. Technol. 10, 47.

Mattox, D. M. (1982). In "Deposition Technologies for Films and Coatings" (R.

F. Bunshah, ed.), Noyes Publ., Park Ridge, New Jersey.

McTaggart, F. K. (1965), Australtan J. Chem. 18, 937, 949.

McTaggart, F. K., (1967), "Plasma Chemistry in Electrical Discharges", Elsevier, Amsterdam, pp. 219-221.

McTaggart, F. K., (1969), Adv. Chem. Ser. 80, 176.

McWhirter, R. W. P. (1965), In "Plasma Diagnostic Techniques". (R. H. Huddlestone and S. L. Leonard, eds.). Ch. 5, pp. 201-264. Academic Press, New York.

Mearns, A. M. (1969) Thin Solid Films $\underline{3}, 201$.

Meiners, L. G. (1982). J. Vac. Sct. Technol. 21, 655.

Millard, M. (1974). In "Techniques and Applications of Plasma Chemistry" ( $J$. R. Hollahan and A. T. Bell, eds.) Wiley, New York, pp. 177-213.

M1llard, M. M. and Kay, E. (1982) J. Electrochem. Soc. 129, 160.

Miller, T. A. (1981) Plasma Chem. Plasma Process. 1, 3.

Miller, T. A., and Bondybey, V. E. (1977). Chem. Phys. Lett. 50, 275.

Mtller, T. A., and Bondybey, V. E. (1980) J. Chem. Phys. 77, 695.

Miller, T. A., Bondybey, V. E., and English, J. H. (1979a) J. Chem. Phys. 70, 2919.

Miller, T. A., Bondybey, V. E., and Zegarsk1, B. R. (1979b) J. Chem. Phys. 70. 4982.

Minkoff, G. J. (1960). "Frozen Free Radicals", Interscience, New York.

Morita, S., Mizutani, T., and Ieda, M., (1971) Japan J. App1. Phys. 10, 1275.

Morita, S., Sawa, G., and Ieda, M. (1976), J. Macromo1. Sc1., 10, 501.

Mosburg, Jr., E. R., Kerns, R. C., and Abeison, J. R. (1983), J. Appl. Phys. 54(9), 4916 .

Morosoff, N., Crist, B., Bumgarner, M., Hsu, T., and Yasuda, H. (1976), J. Macromo1. Sci. 10. 451 .

Morosoff, N., Newton, W., Yasuda, H. (1978) J. Vac. Sc1. Technol. 15(6), 1815. Mukherjee, S. P., and Evans, P. E. (1972) Thin Solid F1lms, 14, 105. 
Nasser, E. (1971). "Fundamentals of Gaseous Ionfzation and Plasma Electronics". Wiley. New York.

Nibler, J. W. (1982). In "Non-Linear Raman Spectroscopy and Its Chemical Applications", NATO Advanced Study Institute Series. (W. Kiefer and D. A. Long, eds.) D. Reidel Publ. Co., Boston.

Nibler, J. W., McDonald, J. R., and Harvey, A. B. (1976) Opt. Commun. 18, 371. Nilnomi, M., and Yanagihara, K., (1979). ACS Symp. Ser. 108, 87.

Dechsner, H. and Gerhard, W. (1972). Phys. Lett. 40a, 211.

O'Hanion, J.F. (1977). In "Oxides and Oxide Films", (A. K. Vijh, ed.). Vol. 5. p. 105. Marcel Dekker, New York.

O'Hanion, J. F., and Pennebaker, W. B. (1971). App 1. Phys. Lett. 18, 554.

O'Hanlon, J. F., and Sampogna, M. (1973). J. Vac. Sci. Technol. 10, 450.

Ojha, S. M. (1982). In "Physics of Thin Films" (G. Hass, M. H. Francombe and J. L. Vossen, eds.). Vol. 12, p. 237. Academic Press, New York.

0'Keefe, J. F., and Lampe, F. W. (1983), App1. Phys. Lett. 42, 217.

olive, G., Pulfrey, D. L., and Young, L. (1972). Thin Solid Films, 12, 427.

0liver, B. M., Clements, R. M., and Smy, P. R. (1970). J. Appl. Phys. 41 . 2117.

Paul, W., Retnhard, H. P., and von Zahn, U. (1958). Z. Physik, 152, 143.

Pealat, M., Taran, J. P. E., Talllet, J., Bacal, M., and Bruneteau, A. M. (1981a) J. App1. Phys. 52, 2687.

Pealat, M., Taran, J. P. E., Talllet, J., Bacal, M., and Bruneteau, A. M. (1981b), Proc. Fifth Intern. Symp. Plasma Chem., Edinburgh, p. 476.

Pellin, M. J., Wright, R. B., and Gruen, D. M. (1981). J. Chem. Phys. 74, 6448 .

Penning, F. M. (1936) Physica, $\underline{3}, 873$.

Perriere, J., Siejka, J., and Chang, R. P. H. (7982). Thin Solid Films, 95, 309 .

Perrin, J., and Delafosse, E. (1980). J. Phys. D. 13, 759.

Perrin, J., and Schmitt, J. P. M. (1982), Chem. Phys. 67, 167.

Podgornyi, I. M. (1971). "Topics in Plasma Dlagnostics", Plenum Press, New York. 
Pol1, H. U., Arzt, M., and Wickleder, K. H. (1976). European Polymer J. 12, 505.

Purdes, A. J., Bolker, B. F. T., Bucci, J. D., and Tisone, T. C. (1977). J. Vac. Sci. Technol. 14, 98.

Rado, W. G. (1967) App 1. Phys. Lett. 11, 123.

Rand, M. J. (1979) J. Vac. Sc1. Technol. 16, 420.

Raveh, A., Inspektor, A., Carmi, U., and Avn1, R. (1983). Thin Solld F1lms, 108, 39 .

Ray, A. K., and Relsman, A. (1981). J. Electrochem. Soc. 128, 2424, 2460, 2466 .

Reinberg, A. R. (1977) Intern. Round Táble Surf. Treat. Plasma Polymer, IUPAC, Limoges, $\mathrm{Fr}$.

Reinberg, A. R. (1979) Ann. Rev. Mater. Sc1. 9, 341; J. Electron. Mater. $\underline{8}$, 345 .

Richter, J. (1968). In "Plasma Dlagnostics". (W. Lochte-Holtgreven, ed.) Ch. 1. pp. 1-65. North-Holland, Amsterdam.

Ron, Y., Raveh, A., Carmi, U., Inspektor, A., and Avn1, R. (1983) Thin Solid Films 107, 183 .

Rosler, R. S., and Engle, G. M. (1979). Soltd State Technol. December issue, p. 88 .

Rosler, R. S., Benzing, W. C., and Baldo, J. (1976) Solid State Technol. $\underline{19}(6), 45$.

de Rosny, G., Mosburg, Jr., E. R., Abelson, J. R., Devaud, G., and Kerns, R. C. (1983), J. App 1. Phys. 54, 2272.

Roth, J. (1982). In "Sputtering by Particle Bombardment" (R. Behrisch, ed.), Vol. II, Springer Verlag, Berlin.

Rowe, B. (1975). Intern. J. Mass Spectrom. Ion Phys.. 16209.

Samsonov, G. V., and Vinitski1, I. M. (1980). "Handbook of Refractory Compounds". IF I/Plenum Pub1. Col., New York.

Schott, L. (1968). In "Plasma Diagnostics" (W. Lochte-Holtgreven, ed.) pp. 668-731. North-Holland, Amsterdam.

Scott, L. (1971). In "Reactions Under Plasma Conditions". (M. Venugopalan, ed.). Vol. I, pp. 515-542, Wiley, New York.

Schwartz, R., and Heinrich, F. (1935), Z. Anorg. Allgem. Chem. 221, 277.

Scott, B. A., Brodsky, M. H., Green, D. C., Kirby, P. B., Placenik, R. M., and Simonyi, E. E. (1980), Appl. Phys. Lett. 37. 725. 
Scott, T. W., Chu, K. C., and Venugopalan, M. (1978) J. Polym. Sci. Poly. Chem. Ed. 16, 3213.

Scott, T. W., Chu, K. C., and Venugopalan, M. (1979). J. Polymer Sci. Poly. Chem. Ed., 17, 267.

Sears, T. J., Mlller, T. A., and Bondybey, V. E. (1980) J. Am. Chem. Soc. 102, 4864 .

Secrist, D. R., and Mackenzie, J. D. (1966a), Bul1. Am. Ceramic. Soc. 45, 784.

Secrist, D. R., and Mackenzie, J. D. (1966b), J. Electrochem. Soc., 113, 914.

Secrist, D. R., and Mackenzfe, J. D. (1969). Adv. Chem. Ser. 80, 242.

Selwyn, G. S., and Kay, E. (1983). Proc. Sixth Intern. Symp. Plasma Chem., Montrea 1. p. 716.

Shahin, M. M. (1969). Advan. Chem. Ser. $\underline{80}, 48$.

Shaub, W. M., Nibler, J. W., and Harvey, A. B. (1977): J. Chem. Phys. 67, 1883.

Shen, M. and Bell, A. T. (1979). In "Plasma Polymerization" (M. Shen and A. T. Bel1, eds.), ACS Symp. Ser. 108, Washington, D.C., pp. 1-33.

Shiloh, M., Gayer, B., and Brinckman, F. E. (1977) J. Electrochem. Soc. 124, 295.

Sigmund, P. (1969). Phys. Rev. 184, 383.

Sinha, A. K., Levinstein, H. J., Smith, T. E., Quintana, G., and Haszko, S. E. (1978) J. Electrochem. Soc. 125, 601 .

Smith, D., and Plumb, I. C. (1973). J. Phys. D. ‥ 1431 .

Smolinsky, G., and Vasile, M. J. (1973) Intern. J. Mass Spectrom. Ion Phys. 12. 47 .

Smolinsky, G., and Vasile, M. J. (1975) Intern. J. Mass Spectrom. Ion Phys. 16. 137 .

Smolinsky, G., and Vasile, M. J. (1976) Intern. J. Mass Spectrom. Ion Phys. 22. 171 .

Smolinsky, G., and Vasile, M. J. (1977). Intern. J. Mass Spectrom. Ion Phys. 24, 11, 311 .

Sokolowski, M. (1979). J. Cryst. Growth, 46, 136.

Sokolowski, M., Sokolowska, A., Michalski, A., Gokieli, B., Romanowski, Z., and Rusek, A. (1977), J. Cryst. Growth, 42, 507.

Sokolowski, M., Sokolowska, A., Michalski, A., Romanowski, Z., Rusek-Mazurek, A., and Wronikowski, M. (1981). Thin Solid Films, 80, 249. 
Sokolowski, M., Sokolowska, A., Rusek, A., Romanowsk1, Z., Gokieli, B., and Gazewska, M. (1981) J. Cryst. Growth, 52, 165.

Sterling, H. F., and Swann, R. C. G. (1965) Solld-State Electron. 8, 653.

Sterling, H. F., Alexander, J. H., and Joyce, R. J. (1968) Special Ceram. 4, 139.

Stern, R. A. (1977). Phys. Fluids, 21, 1287.

Stuart, R. V., and Wehner, G. K. (1962), J. App 1. Phys., 33, 2345.

Suhr. H. (1974). In "Techniques and Applications of Plasma Chemistry" (J. R. Hollahan and A. T. Be11, eds.) Wtley, New York, pp. 63-66.

Swann, R. C. G., Mehta, R. R., and Cauge, T. P. (1967) J. Electrochem. Soc. $114,713$.

Swift, J. D. (1969). Brit. J. Appl. Phys. Ser. 2, 2, 134.

Swift, J. D., and Schwar, M. J. R. (1969). "Electrical Probes for Plasma Diagnostics". American Elsevier. New York.

Szabo, A., and Withelmi, H. (1983). Proc. Sixth Intern. Symp. Plasma Chem., Montrea 1, p. 346.

Taft, E. A. (1971) J. Electrochem. Soc., 118, 1341.

Taniguchi, M., Hirose, M., Hamasak1, T., and Osaka, Y. (1980). Appl. Phys. Lett. $\underline{37}, 787$.

Taylor, J. A., Lancaster, G. M., Ignatiev, A., and Rabalais, J. W. (1978). J. Chem. Phys. 68, 1776.

Thenard, P., and Thenard, A. (1874) C. R. Acad. Sc1. 78, 219.

Thompson, L. F., and Mayhan, K. G. (1972) J. App1. Polym. Sc1. 16, 2317.

Thornton, J. A. (1978). J. Vac. Sc1. Technol. 15(2), 188.

Thornton, J. A. (1982). In "Deposition Technologies for Films and Coatings" (R. F. Bunshah, ed.). Noyes Publ., Park Ridge, New Jersey.

Thornton, J. A., and Penfold, A. S. (1978). In "Thin F1lm Processes" (J. L. Vossen and W. Kern, eds.). Academic Press, New York, pp. 75-113.

Tlbbetts, G. G. (1974) J. Appl. Phys. 45, 5072.

Tibbitt, J. M., Jensen, R., Bell, A. T., and Shen, M. (1977). Macromol. 10, 647.

Tiller, H. J., Berg, D., and Mohr, R. (1981) Plasma Chem. Plasma Process. 1, 247.

Tkatschuk, B. W., Ganulk, L. J., and Laurs, E. P. (1977). Khim. Vys. Energ. 11. 350 . 
Turban, G., Catherine, Y., and Grolleau, B. (1979) Thin Solid F1lms, 60, 147.

Turban, G., Catherine, Y., and Grolleau, B. (1982) Plasma Chem: Plasma Process. 2, 61 .

Turner, E. B. (1965). In "Plasma Diagnostics Techniques". (R. H. Huddlestone and S. L. Leonard, eds.). Ch. 7, pp. 319-358. Academic Press, New York.

Vasile, M. J., and Smolinksy, G. (1973) Intern. J. Mass Spectrom. Ion Phys. 12. $133 ; 13,381$.

Vasile, M. J., and Smolinsky, G. (1975) Intern. J. Mass Spectrom. Ion Phys. 18. 179.

Vasile, M. J., and Smolinsky, G. (1977) Intern. J. Mass Spectrom. Ion Phys. 24, 11 .

Vastle, M. J., and Smolinsky, G. (1977) J. Phys. Chem. 81, 2605.

Venugopalan, M. (ed.) (1971). "Reactions Under Plasma Conditions", Vol. I, II, Wiley, New York.

Venugopalan, M. (1983) Plasma Chem. Plasma Process. $\underline{3}, 275$.

Venugopalan, M., and Jones, R. A. (1968). "Chemistry of Dissociated Water Vapor and Related Systems", Wiley, New York.

Venugopalan, M., and Veprek, S. (1983). In "Plasma Chemistry IV" (S. Veprek and M. Venugopalan, eds.), Springer Verlag, Berlin, pp. 1-58.

Venugopalan, M., Lin, I. S., and Grenda, M. S. (1980), J. Polym Sci., Poly. Chem. Ed., 18, 2731.

Veprek, S. (1980). Curr. Top. Mater. Sc1. 4, 151.

Veprek, S. (1982). Pure App1. Chem., 54, 1197.

Veprek, S., and Marecek, V. (1968). Sol1d State Electron. 11, 683.

Veprek, S., and Roos, J. (1976) J. Phys. Chem. Solids, 37, 554.

Veprek, S., Brende1, C., and Schafer, H. (1971) J'. Cryst. Growth, 9, 266.

Veprek, S., Cocke, D. L., and Gingerich, K. A. (1975). Chem. Phys. I, 294.

Veprek, S., Iqbal, Z., Oswald, H. R., and Webb, A. P. (1981), J. Phys. C., 14, 295.

Veprek, S., Iqbal, Z., Bunner, J., and Scharl1, M. (1981) Ph11. Mag. B43, 527.

Vinogradov, G. K., Ivanov, Yu. A., and Polak, L. (1981) High Energy Chem. 15, 120.

Vinogradov, G. K., Nevzorov, P. I., Polak, L. S., and Slovetsky, D. I. (1982). Vacuum $\underline{32}$. 529. 
Vossen, J. L. (1979). J. Electrochem. Soc. 126, 319.

Vossen, J. L., and Cuomo, J. J. (1978). In "Thin F1lm Processes" (J. L. Vossen and W. Kern, eds.) Academtc Press, New York, pp. 11-73.

Wager, J. F., Makky, W. H., Wilmsen, C. W., and Meiners, L. G. (1982). Thin Solid Films, $\underline{95}, 343$.

Wagner, J. J., and Brandt, W. W. (1981). Plasma Chem. Plasma Process. 1, 201. Wagner, J. J., and Veprek, S. (1982). Plasma Chem. Plasma Process. 2, 95. Wagner, J. J., and Veprek, S. (1983). Plasma Chem. Plasma Process. 3 , 219 . Watts, R. K. (1978). In "Thin Films Processes" (J. L. Vossen and W. Kern, eds.). Academic Press, New York, pp. 131-174.

Walkup, R., Dreyfus, R. W., and Avouris, Ph. (1983). Proc. Sixth Intern. Symp. Plasma Chem., Montreal, p. 701.

Weinretch, 0. A. (1966). J. Appl. Phys. 37, 2924.

Westenberg, A. A. (1965). J. Chem. Phys. $\underline{43}, 1544$.

Westenberg, A. A. (1969). Science, 164, 381.

Westwood, A. R. (1971) Eur. Polym. J. I, 363.

de Wilde, P. (1874). Ber. Dtsch. Chem. Ges. I, 4658.

Williams, T., and Hayes, M. W. (1967). Nature (London), 216, 614.

Winters, H. F. (1976). Adv. Chem. Ser. 158, 1 .

Winters, H. F. (1980), In "Plasma Chemistry III". (S. Veprek and M. Venugopalan, eds.), pp. $\overline{69}-125$, Springer Verlag, Berlin.

Woodyard, J. R., and Cooper, C. B. (1964). J. App1. Phys. 35, 1107.

Wright, R. B., Pellin, M. J., and Gruen, D. M. (1981) Surf. Sci. 110, 151.

Wright, R. B., Young, C. E., Pellin, M. J., and Gruen, D. M. (1982). J. Vac. Sci. Technol. 20(3), 510 .

Yamagucht, S., Sawa, G., and Ieda, M. (1977), J. App1. Phys. 48, 2363.

Yamaguch1, S., Sawa, G., and Ieda, M. (1979), ACS Symp. Ser. 108, 115.

Yamasaki, K., and Sugano, T. (1980). J. Vac. Sci. Technol. 17, 959.

Yanagihara, K. and Yasuda, H. (1982) J. Polymer Sci., Polymer Chem. Ed. 20, 1833.

Yanagihara, K. and Nitinomi, M. (1983) Proc. Intern. Ion Eng. Congr., Kyoto, Sep. 1983, p. 1475. 
Yanagihara, K., Katuta, T., Ara1, K., and Nilnomi, M. (1981). Kobunshi Ronbunshu $38(10), 741$.

Yanagthara, K., Arai, K., and Nifnomi, M. (1982a). Preprint ACS National Meeting, Div. Org. Coating and Plastics Chem., Kansas City, Sep. 1982, p. 304.

Yanagihara, K., Aral, K., Nilnomi, M., and Yasuda, H. (1982b). Preprint ACS National Meeting. Div. Org. Coating and Plastic Chem., Kansas City, Sep. 1982, p. 217.

Yasuda, H. (1976). In "Plasma Chemistry of Polymers" (M. Shen, ed.), p. 21, 39, Marcel Dekker, Inc., New York.

Yasuda, H. (1977) Intern. Round Table Plasma Polymer. Treat. IUPAC Symp. Plasma Chem. Limoges, Fr.

Yasuda, H. (1978). In "Thin F11m Processes" (J. L. Vossen and W. Kern, eds.) pp. 360-398. Academic Press, New York.

Yasuda, H. (1979), ACS Symp. Ser. 108, 37.

Yasuda, H. and Hsu, T. (1977) J. Polym. S1., Poly. Chem. Ed. $\underline{5}, 81$.

Yoshihara, H., Mori, H., and Kiuchi, M. (1981) Thin Solid F1lms, 76, 1.

Yoshihara, H., Mori, H., Kiuch1, M., and Kadota, T. (1978). Japan J. Appl. Phys. 17. 1693.

Zesch, J. G., Lujan, R. A., and Deline, V. R., (1980), J. Non-Cryst. Solids, $\underline{35} / \underline{36}, 273$.

Zlemann, P. and Kay, E. (1982) J. Vac. Sc1. Technol. 21, 828.

Zlemann, P., Koehler, K., Coburn, J. W., and Kay, E. (1983), J. Vac. Sci. Technol. B1(1), 31 . 
TABLE I. - SPUTTERING YIELDS FOR ARGON ION BOMBAROMENT AT VARIOUS ENERGIES ${ }^{\mathrm{a}}$

\begin{tabular}{|ll|l|l|l|r|r|}
\hline \multicolumn{2}{|c|}{$\begin{array}{c}\text { Threshold, } \\
\mathrm{eV}\end{array}$} & \multicolumn{5}{|c|}{$\mathrm{AR}^{+} / \mathrm{eV}$} \\
\cline { 3 - 7 } & & 60 & 100 & 200 & 300 & 600 \\
\hline $\mathrm{Ag}$ & 15 & 0.22 & 0.63 & 1.58 & 2.20 & 3.40 \\
$\mathrm{Al}$ & 13 & & .11 & .35 & .65 & 1.24 \\
$\mathrm{Au}$ & 20 & & .32 & 1.07 & 1.65 & 2.43 \\
$\mathrm{Be}$ & 15 & & .074 & .18 & .29 & .80 \\
$\mathrm{Cr}$ & 22 & & .30 & .67 & .87 & 1.30 \\
$\mathrm{Cu}$ & 17 & .10 & .48 & 1.10 & 1.59 & 2.30 \\
$\mathrm{Fe}$ & 20 & .064 & .20 & .53 & .76 & 1.26 \\
$\mathrm{Ge}$ & 25 & & .22 & .50 & .74 & \\
$\mathrm{Mo}$ & 24 & .027 & .13 & .40 & .58 & .93 \\
$\mathrm{Nb}$ & 25 & .017 & .068 & .25 & .40 & .65 \\
$\mathrm{NI}$ & 21 & .067 & .28 & .66 & .95 & 1.52 \\
$\mathrm{Pd}$ & 20 & & .42 & 1.00 & 1.41 & 2.39 \\
$\mathrm{Pt}$ & 25 & .032 & .20 & .63 & .95 & 1.56 \\
$\mathrm{Re}$ & 35 & & .10 & .37 & .56 & .91 \\
$\mathrm{Si}$ & & & .07 & .18 & .31 & .53 \\
$\mathrm{Ta}$ & 26 & .01 & .10 & .28 & .41 & .62 \\
$\mathrm{TH}$ & 20 & & .081 & .22 & .33 & .58 \\
$\mathrm{~V}$ & 23 & .03 & .11 & .31 & .41 & .70 \\
$\mathrm{~W}$ & 33 & .008 & .068 & .29 & .40 & .62 \\
$\mathrm{Zr}$ & 22 & .027 & .12 & .28 & .41 & .75 \\
\hline
\end{tabular}

a The yield $S$ is given as $S /(1+\gamma)$, where $\gamma$ is the secondary electron emission coefficient. Data collated from N. Laegreid and G. K. Wehner, J. Appl. Phys. 32, 365(1961) and R. V. Stuart and G. K. Wehner, J. Appl. Phys. 33 , 2345(1962), by permission of the American Institute of Physics.

TABLE II. - IONIZATION DEGREE AND POLYMERIZATION DEGREE IN A 16 VOI $\$ \mathrm{C}_{3} \mathrm{H}_{6}+\mathrm{Ar} \mathrm{rf}$ PLASMA BULK AND IN PLASMA LAYER (GRAPHITE SUBSTRATE) $^{a, b}$

\begin{tabular}{|c|c|c|}
\hline \multicolumn{3}{|c|}{ Pressure 1 to 6 torr } \\
\hline \multicolumn{3}{|c|}{ Power $150 \mathrm{~W}$ at $13.56 \mathrm{MHz}$} \\
\hline \multirow{3}{*}{$\begin{array}{l}n_{j} / n \\
n_{p o I y} / n\end{array}$} & Plasma Bulk & Plasma Layer \\
\hline & $3 \times 10^{-5}$ & $5 \times 10^{-3}$ \\
\hline & $2 \times 10^{-1}$ & $>8 \times 10^{-1}$ \\
\hline \multicolumn{3}{|c|}{$\begin{array}{l}\text { afrom A. Inspektor, U. Carmi, R. Avni } \\
\text { and H. Nickel, Piasma Chem. Piasma } \\
\text { Process. I, 377(1981), by permission } \\
\text { of Plenum Publishing Corporation. } \\
\text { beasurements made in position } F \text { with } \\
\text { respect to figure } 39 \text {, } 1 . e . \text { a position } \\
\text { immediately beyond the plasma. n is } \\
\text { the concentration of } \mathrm{C}_{3} \mathrm{H}_{6} \text { in the } \\
\text { gas mixture. }\end{array}$} \\
\hline
\end{tabular}


TABLE III. - SPECTRA OBSERVED IN GLOW DISCHARGEa

\begin{tabular}{|c|l|l|l|}
\hline Particle & \multicolumn{1}{|c|}{ Degree of freedom } & Type of spectrum & Spectral region \\
\hline Atom or lon & Electronic excitation & Line & uv-visible-ir \\
& Ionization & Continuum & uv-visible-ir \\
& Translation & Line profiles & \\
Electrons & Recombination & Continuum & uv-visible \\
& Free-free transitions & Continuum & ir \\
& Rotation & Line & Far ir \\
& Vibration-rotation & Band & ir \\
& Electronic exictation & Band systems & uv-visible-ir \\
\hline
\end{tabular}

afrom Cabannes F., and Chapelle, 3. (1971). In "Reactions Under plasma Conditions" (M. Venugopalan, ed.). Vol. I, Pp. 367 to 469. Wiley-Interscience, New York.

TABLE IV. - ELECTRON TEMPERATURE $\left(T_{e}\right)$, POSITIVE ION DENSITY $\left(n_{1}\right)$, AND DEGREE OF IONIZATION AT TWO RADIOFREQUENCIES ${ }^{a}$

[After Grossman et al., 1982.]

\begin{tabular}{|l|c|c|c|c|c|c|}
\hline \multirow{2}{*}{$p / m b a r$} & \multicolumn{2}{|c|}{$T_{\mathrm{e}} / \mathrm{eV}$} & \multicolumn{2}{c|}{$\mathrm{n}_{1} \times 10^{-11} \mathrm{~cm}^{-3}$} & \multicolumn{2}{c|}{ Degree of Ionization $\times 10^{4}$} \\
\cline { 2 - 7 } & $0.4 \mathrm{MHz}$ & $27.12 \mathrm{MHz}$ & $0.4 \mathrm{MHz}$ & $27.12 \mathrm{MHz}$ & $0.4 \mathrm{MHz}$ & $27.12 \mathrm{MHz}$ \\
\hline 0.5 & 4.48 & 4.74 & 0.5 & 3.7 & 0.10 & 0.80 \\
1.0 & 5.17 & 5.43 & 0.8 & 7.0 & .09 & .73 \\
3.0 & 5.00 & 4.48 & 1.0 & 9.8 & .03 & .34 \\
5.0 & 3.10 & 2.58 & 1.5 & 10.7 & .03 & .22 \\
\hline
\end{tabular}

a Gas mixture $A r+10$ vol $\& \mathrm{H}_{2}$; substrate in the center of the glow; current in the of coil $12 \mathrm{~A}(0.4 \mathrm{MHz})$ and $4 \mathrm{~A}(27.12 \mathrm{MHz})$. 
TABLE V. - ELECTRONIC TRANSITIONS OBSERVED IN THE EMISSION SPECTRA OF OF NITRIDING ( $r f$ ) PLASMAS

[After Matsumoto and Kanzak1, 1983.]

\begin{tabular}{|c|c|c|c|}
\hline Plasma & Speci & ransitions & $T_{v} / K$ \\
\hline $\begin{array}{l}\mathrm{N}_{2} / \mathrm{H}_{2} \\
: 9 / 1\end{array}$ & $\begin{array}{l}N_{2}, B^{3} \pi_{g}-A^{3} \Sigma_{g}^{+} \\
N_{2}, C^{3} \pi_{u}-B^{3} \pi_{g} \\
N_{2}, D^{3} \Sigma_{u}^{+}-B^{3} \pi_{g} \\
N_{2}^{+}, B^{2} \Sigma_{u}^{+}-X^{2} \Sigma_{g}^{+} \\
N_{2}^{+}, D^{2} \pi_{g}-A^{2} \pi_{u} \\
N O, A^{2} \Sigma^{+}-X^{2} \pi \\
N_{2}, C^{3} \pi_{u}-B^{3} \pi_{g} \\
N_{2}, D^{3} \Sigma_{u}^{+}-B^{3} \pi_{g} \\
N_{2}^{+}, B^{2} \Sigma_{u}^{+}-X^{2} \Sigma_{g}^{+} \\
N H, A^{3} \pi-X^{3} \Sigma^{-} \\
H, H_{\alpha} \cdot H_{B}, H_{Y}\end{array}$ & $\begin{array}{l}\Delta v=3,4\left(v^{\prime}=13\right) \\
\Delta v=-7 \text { to }+3\left(v^{\prime}=11\right) \\
\Delta v=-4 \text { to }-1\left(v^{\prime}=4\right) \\
\Delta v=-2 \text { to }+2\left(v^{\prime}=4\right) \\
\Delta v=-2 \text { to }+2\left(v^{\prime}=7\right) \\
\Delta v=-5 \text { to }+2\left(v^{\prime}=6\right) \\
\Delta v=-7 \text { to }+3\left(v^{\prime}=11\right) \\
\Delta v=-4 \text { to }-1\left(v^{\prime}=4\right) \\
\Delta v=0(0-0,1-1) \\
\Delta v=0(0-0,1-1)\end{array}$ & $\begin{array}{l}5400(\Delta v=-2) \\
4400(\Delta v=+1) \\
5700(\Delta v=-1)\end{array}$ \\
\hline
\end{tabular}


TABLE VI. - POSITIVE IONS FROM AN $\mathrm{rf}$ PLASMA IN AT/C $6 \mathrm{H}_{6}$ UNOER CONDITIONS OF GOOD FILM DEPOSITION

[After N1inomi and Yanagihara (1978).]

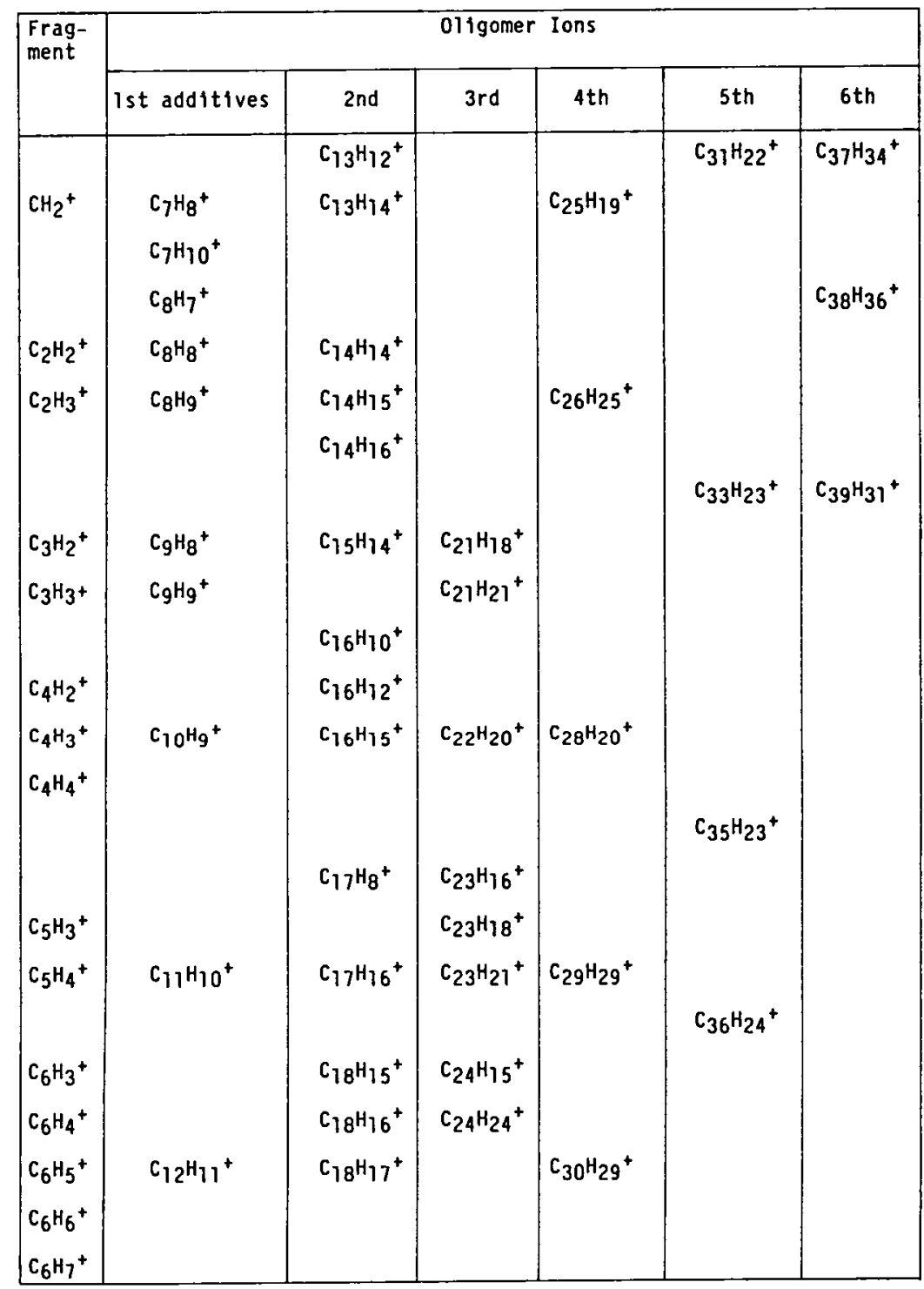


TABLE VII. - ELECTRON DENSITY IN AN Ar $+\mathrm{C}_{3} \mathrm{H}_{6}$ PLASHA MEASURED BY DIFFERENT METHODS ${ }^{a} \cdot b^{6}$

\begin{tabular}{|c|c|c|c|c|}
\hline \multirow{2}{*}{$\begin{array}{c}\text { Pressure } \\
\text { torr }\end{array}$} & \multicolumn{4}{|c|}{$\mathrm{n}_{\mathrm{e}} \times 10^{-11} \mathrm{~cm}^{-3}$} \\
\cline { 2 - 5 } & DFPS & $\sigma_{\mathrm{e}}$ & \multicolumn{2}{|c|}{ Stark broadening } \\
\cline { 4 - 5 } & & & $\mathrm{H}_{\mathrm{B}}$ & $\mathrm{H}_{\mathrm{n}=11}$ \\
\hline 1 & 2.4 & 1.5 & 15.0 & 4.2 \\
3 & 3.5 & 3.0 & 17.8 & 5.6 \\
5 & 4.5 & 3.7 & 20.5 & 8.0 \\
7 & 7.8 & 6.1 & 23.2 & - \\
11 & 10.5 & 91.4 & 30.3 & - \\
\hline
\end{tabular}

afrom A. Inspektor, U. Carmi, R. Avnt and $H$. Nickel, Plasma Chem. Plasma Process. 1, 377(1981), by permission of Plenum Publishing Corporation. bMeasurements made in Position G (fig. 39); Power = $100 \mathrm{w} ; \mathrm{Ar} / \mathrm{C}_{3} \mathrm{H}_{6}=3$.

TABLE VIII. - NORMALIZED VALUES OF ELECTRON EXCITATION FUNCTIONS $f\left(n_{e}\right)$, RAOICAL DENSITIES, AND POLYMER DEPOSITION RATES,

$r_{p}\left(\mathrm{~g} \mathrm{~cm}^{-2} \mathrm{~min}^{-1}\right)$, IN PURE $\mathrm{C}_{2} \mathrm{~F}_{4}$ AND

IN IN MIXTURES OF $C_{n} F_{2 n+2}+20 \%$ OF ADDITIVES

[After R. d'Agostino et a1., 1983.]

\begin{tabular}{|l|c|c|c|c|}
\hline \multicolumn{1}{|c|}{ Feed } & $f\left(\mathrm{n}_{\mathrm{e}}\right)$ & \multicolumn{1}{c|}{$\mathrm{CF}$} & $\mathrm{CF}_{2}$ & $\mathrm{r}_{\mathrm{p}}$ \\
\hline $\mathrm{CF}_{4}-\mathrm{H}_{2}$ & 1.0 & 0.13 & 0.004 & 1.3 \\
$\mathrm{CF}_{4}-\mathrm{C}_{2} \mathrm{~F}_{4}$ & .41 & .20 & .20 & 1.2 \\
$\mathrm{C}_{2} \mathrm{~F}_{6}-\mathrm{H}_{2}$ & .45 & .10 & .20 & 2.9 \\
$\mathrm{C}_{3} \mathrm{~F}_{8}-\mathrm{H}_{2}$ & .20 & .08 & .05 & 2.1 \\
$\mathrm{C}_{2} \mathrm{~F}_{4}$ & .06 & 1.0 & 1.0 & .9 \\
\hline
\end{tabular}


TABLE IX. - INORGANIC FILMS DEPOSITED BY PCVD TECHNIQUE

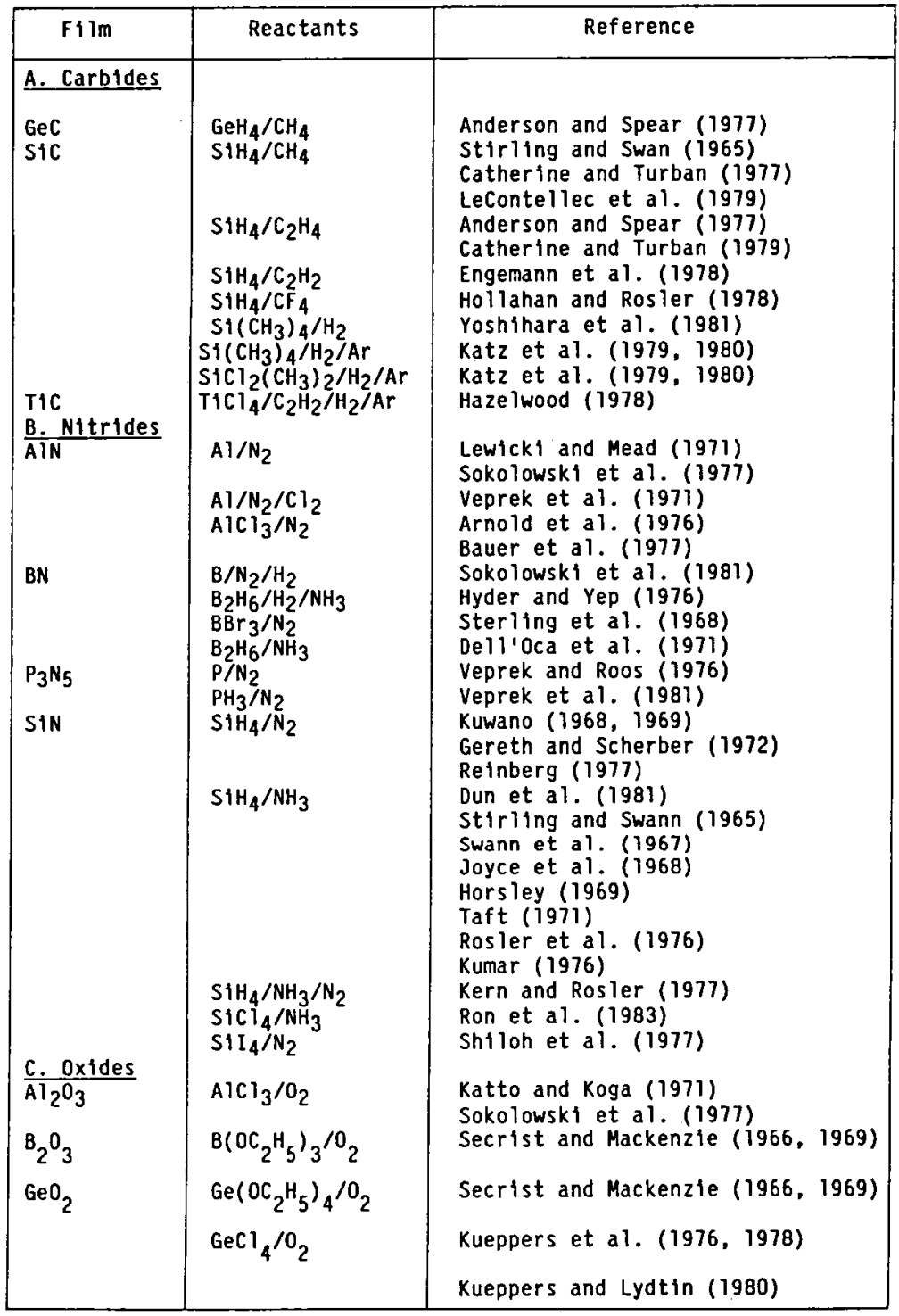


TABLE IX. - Continued.

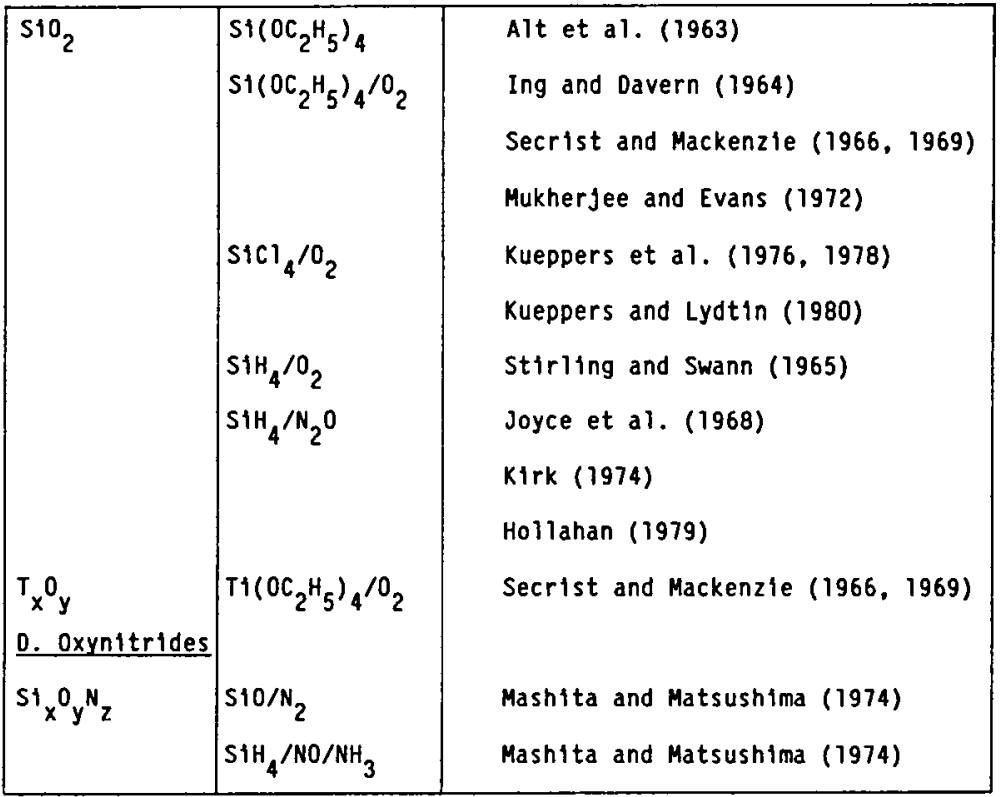

TABLE X. - GIBBS ENERGY OF FORMATION AT 773 K FOR REFRACTORY COMPOUNDSa $\Delta G$ at $773 \mathrm{~K}, \mathrm{kal} \mathrm{mol}^{-1}$

\begin{tabular}{|c|c|c|c|c|c|}
\hline \multicolumn{2}{|l|}{ Boriding } & \multicolumn{2}{|l|}{ Nitriding } & \multicolumn{2}{|l|}{ Carburizing } \\
\hline $\begin{array}{l}\mathrm{TH}+2 \mathrm{~B}=\mathrm{THB}_{2} \\
2 \mathrm{~T} 1+5 \mathrm{~B}=\mathrm{TH}_{2} \mathrm{~B}_{5} \\
\mathrm{~V}+2 \mathrm{~B}=\mathrm{VB}_{2} \\
\mathrm{CR}+2 \mathrm{~B}=\mathrm{CrB}_{2}\end{array}$ & $\begin{array}{l}-68.0 \\
-99.5 \\
-60.0 \\
-30.2\end{array}$ & $\begin{array}{l}\mathrm{Ti}+(1 / 2) \mathrm{N}_{2}=\mathrm{TIN} \\
\mathrm{V}+(1 / 2) \mathrm{N}_{2}=\mathrm{VN} \\
4 \mathrm{Cr}+(1 / 2) \mathrm{N}_{2}=2 \mathrm{Cr}_{2} \mathrm{~N} \\
\mathrm{~B}+(1 / 2) \mathrm{N}_{2}=\mathrm{BN} \\
\mathrm{A} 1+(1 / 2) \mathrm{N}_{2}=\mathrm{AlN} \\
3 \mathrm{SI}+2 \mathrm{~N}_{2}=\mathrm{SI}_{3} \mathrm{~N}_{4}\end{array}$ & $\begin{array}{l}-63.0 \\
-43.5 \\
-26.5 \\
-18.5 \\
-59.8 \\
-117.8\end{array}$ & $\begin{array}{l}T 1+C=T 1 C \\
V+C=V C \\
7 \mathrm{Cr}+3 \mathrm{C}=\mathrm{Cr}_{7} \mathrm{C}_{3} \\
2 \mathrm{Fe}+\mathrm{C}=\mathrm{Fe}_{2} \mathrm{C} \\
4 \mathrm{~B}+\mathrm{C}=\mathrm{B}_{4} \mathrm{C} \\
\mathrm{Si}+\mathrm{C}=\mathrm{S} 1 \mathrm{C}\end{array}$ & $\begin{array}{l}-41.9 \\
-11.3 \\
-46.4 \\
+2.0 \\
-10.9 \\
-11.5\end{array}$ \\
\hline
\end{tabular}

Data collated from Handbook of Refractory Compounds, by G. V. Samsonov and

I. M. Vinitskif, If I/Plenum Publishing Co., New York, 1980. 
TABLE XI. - KINETICS OF REACTIONS IN PLASMA SURFACE INTERACTIONS (PSI)

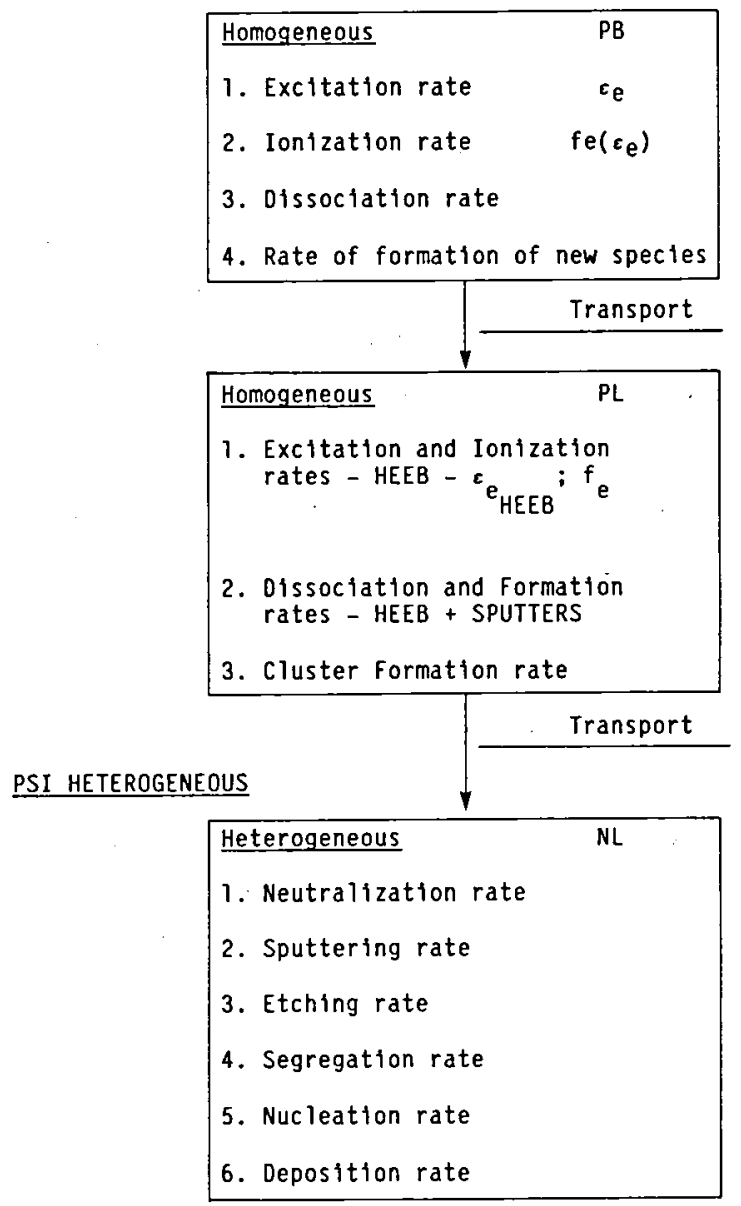

TABLE XII. - FLUXES AND RATES DF SPUTTERING, ETCHING AND DEPOSITION OF SILICON IN A MICROWAVE (2.45 GHz) PLASMA

\begin{tabular}{|c|c|c|c|c|c|c|}
\hline \multicolumn{4}{|c|}{$\begin{array}{l}\text { Kinetic Energy of } \mathrm{Ar}^{+}=600 \mathrm{eV} \\
\mathrm{J}_{\mathrm{Ar}^{+}}=3.4 \times 10^{16} \mathrm{~cm}^{-2} \mathrm{~s}^{-1} \\
5 \times 10^{-2} \leq \mathrm{P} \leq 2.0 \text { torr }\end{array}$} & \multicolumn{3}{|c|}{$\begin{array}{l}\mathrm{n}_{1}\left(\mathrm{Ar}^{+}\right)=10^{11} \mathrm{~cm}^{-3} \\
\mathrm{~J}_{\mathrm{F}_{2}}=\mathrm{J}_{\mathrm{Cl}_{2}}=10^{16} \mathrm{~cm}^{-2} \mathrm{~s}^{-1} \\
\text { si substrate grounded }\end{array}$} \\
\hline & , & . & & $\mathrm{S1}$ & & . \\
\hline \multirow[t]{2}{*}{ Plasma } & Sputter & \multicolumn{2}{|c|}{ Etcha } & \multicolumn{3}{|c|}{ Depositionb } \\
\hline & Ar & $A r+F_{2}$ & $\mathrm{Ar}+\mathrm{Cl}_{2}$ & $\mathrm{Ar}+\mathrm{SiF}_{4}$ & $\mathrm{Ar}+\mathrm{SiCl}_{4}$ & $\mathrm{Ar}+\mathrm{SICl}_{4}+\mathrm{H}_{2}$ \\
\hline Flux $=10^{16} \mathrm{~cm}^{-2} \mathrm{~s}^{-1}$ & 1.6 & 9.5 & 1.9 & $1.0^{\mathrm{C}}$ & $1.2^{\mathrm{C}}$ & $2.8^{\mathrm{C}}$ \\
\hline Rate, $\mathrm{nms}^{-1}$ & - & 0.08 & 0.03 & 1.2 & 1.22 & 1.55 \\
\hline
\end{tabular}

aflux data from Winters (1980) and Vinogradov et al, (1982); rate data on single crystal from Chapman (1980) and Vinogradov et al. (1982).

bMtcrocrystalline 51 film deposited at 0.5 and 2.0 torr from 5 vol $\% \mathrm{SiF}_{4}$ in $\mathrm{Ar}$ (Avni, unpublished), 5 vol $\mathscr{S} \mathrm{SiF}_{4}$ in Ar (data from Avni (1984) and Avni et

al. (1983b)), and 5 vol\% $\mathrm{StCl}_{4}+15$ vol $\% \mathrm{H}_{2}$ in Ar (data from Avni (1984)

and Avni et al. $(1983 a, b))$.

calculated from the ISi/EI normalized concentration as measured by mass spectrometry (data from Avni (1984) and Avni et al. (1983b)). 
TABLE XIII. - REACTION MECHANISM FOR PLASMA-POLYMERIZATION

OF UNSATURATED HYDROCARBONS ${ }^{\mathrm{a}}$

Initiation

1. e $+M_{g} \rightarrow M_{g}{ }^{\prime}+H_{2}+e$

2. $e+M_{g} \rightarrow M_{g}{ }^{\prime}+2 H+e$

3. $e+M_{g} \rightarrow 2 R_{g}+e$

4. $\mathrm{e}+\mathrm{H}_{2} \rightarrow 2 \mathrm{H}+\mathrm{e}$

Propagation (Homogeneous)

5. $H+\left\{\begin{array}{l}M_{g} \\ M_{g}^{\prime}\end{array}\right\} \rightarrow R_{g_{1}}$

6. $R_{g_{n}}+\left\{\begin{array}{l}M_{g} \\ M_{g}^{\prime}\end{array}\right\} \rightarrow R_{g_{n+1}}$

Adsorption

7. $S+\left\{\begin{array}{l}M_{g} \\ M_{g}\end{array}\right\} \rightarrow\left\{\begin{array}{l}M_{s} \\ M_{s}\end{array}\right\}$

8. $\mathrm{S}+\mathrm{H} \rightarrow \mathrm{H}_{5}$

9. $S+R_{g_{n}} \rightarrow R_{S}$
Propagation (Heterogeneous)

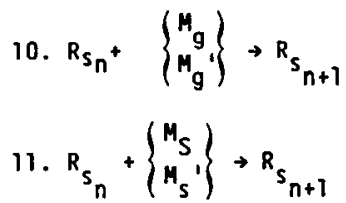

Termination

12. $\mathrm{H}_{5}+\mathrm{H} \rightarrow \mathrm{H}_{2}$

13. $\mathrm{R}_{\mathrm{g}_{\mathrm{m}}}+\mathrm{H} \rightarrow \mathrm{P}_{\mathrm{g}_{\mathrm{m}}}$

14. $R_{g_{m}}+R_{g_{n}} \rightarrow P_{g_{m+n}}$

15. $R_{g_{m}}+R_{s_{n}} \rightarrow P_{s_{m+n}}$

16. $R_{s_{m}}+R_{n} \rightarrow P_{s_{m+n}}$

$\frac{\text { Reinitiation }}{17 . e+P_{g_{m+n}}} \rightarrow R_{g_{m}}+R_{g}$

18. $\mathrm{P}_{\mathrm{s}} \stackrel{\mathrm{e}, \mathrm{hu}, \mathrm{I}^{+}}{\longrightarrow} \mathrm{R}_{\mathrm{s}_{\mathrm{m}}}+\mathrm{R}_{\mathrm{s}_{\mathrm{n}}}$

19. $H+P_{g_{n}} \rightarrow R_{g_{n}}+H_{2}$

20. $H+P_{s_{n}} \rightarrow R_{s_{n}}+H_{2}$

${ }^{a}$ From J. M. Tibbit, R. Jensen, A. T. Bell, and M. Shen, Macromol. 10, 647(1977), by permission of the American Chemical Society.

TABLE XIV. - OVERALL REACTION RATE CONSTANTS $\left(k_{0}\right)$ FOR THE DISSOCIATION OF $\mathrm{C}_{3} \mathrm{H}_{6}, \mathrm{SiH}_{4}$, AND $\mathrm{SICl}_{4}$

Input power $=100 \mathrm{~W}$

Position $H-G$

Pressure $=1.0$ torr

Overall reaction $t$ ime $\left(t_{0}\right)=2.2 \mathrm{~ms}$

\begin{tabular}{|c|c|c|c|c|}
\hline \multirow[t]{3}{*}{ Gas mixture, vol\% } & \multicolumn{3}{|c|}{ Plasma } & \multirow{3}{*}{$k_{0}=\frac{k_{0}\left(\mathrm{Ar}+\mathrm{H}_{2}\right)\left(\mathrm{NH}_{3}\right)}{k_{0}(\mathrm{Ar})}$} \\
\hline & $\mathrm{C}_{3} \mathrm{H}_{6}$ & $\mathrm{SiH}_{4}$ & $\mathrm{SiCl}_{4}$ & \\
\hline & \multicolumn{3}{|c|}{$\begin{array}{l}\text { Overall reaction rate } \\
\text { constant, } k_{0}, s^{-1}\end{array}$} & \\
\hline $16 \mathrm{C}_{3} \mathrm{H}_{6}+\mathrm{Ar}$ & 228 & - & - & 0.26 \\
\hline $66 \mathrm{C}_{3} \mathrm{H}_{6}+\mathrm{Ar}$ & 60 & - & - & .26 \\
\hline $5.0 \mathrm{SiH}_{4}+\mathrm{Ar}$ & - & 330 & - & .29 \\
\hline $5.0 \mathrm{SiH}_{4}+15 \mathrm{H}_{2}+\mathrm{Ar}$ & - & 95 & - & .29 \\
\hline $3.5 \mathrm{SiCl}_{4}+\mathrm{Ar}$ & - & - & 15 & 40.0 \\
\hline $3.5 \mathrm{SiCl}_{4}+20 \mathrm{H}_{2}+\mathrm{Ar}$ & - & - & 600 & 40.0 \\
\hline $3.5 \mathrm{StCl}_{4}+15 \mathrm{NH}_{3}+\mathrm{Ar}$ & - & - & 400 & 27.0 \\
\hline
\end{tabular}

afrom R. Avni, U. Carmi, A. Inspektor, and I. Rosenthal, NASA Tech. Paper-2301, April 1984. 
TABLE XV. - MONOMER COMSUMPIION (dM/dt) AND DEPOSITION RATE $(d G / d t)$ IN $\mathrm{rf}$ PLASMAS [Reactor cross section $80 \mathrm{~cm}^{2}$.]

\begin{tabular}{|c|c|c|c|c|c|}
\hline \multirow{2}{*}{$\begin{array}{c}\text { Monomer in } \\
\text { gas mixtures } \\
\text { vols }\end{array}$} & \multirow{2}{*}{$\begin{array}{l}\text { Monomer feed } \\
\mathrm{mg} \mathrm{cm}-2 \mathrm{~s}^{-1}\end{array}$} & \multirow{2}{*}{ 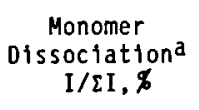 } & \multicolumn{2}{|c|}{$\mathrm{mg} \mathrm{cm} \mathrm{cm}^{-2} \mathrm{~s}^{-1}$} & \multirow[t]{2}{*}{$\bar{v}_{e f f} / v(g)$} \\
\hline & & & $\mathrm{dM} / \mathrm{dt}$ & $\mathrm{dG} / \mathrm{dt}$ & \\
\hline $\mathrm{b}_{5} \mathrm{SiCl}_{4}+20 \mathrm{H}_{2} / \mathrm{Ar}$ & 0.30 & 85 & 0.25 & 0.10 & 0.40 \\
\hline 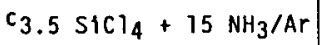 & .19 & 80 & .15 & .07 & .46 \\
\hline $\mathrm{d}_{16} \mathrm{C}_{3} \mathrm{H}_{6} / \mathrm{Ar}$ & .35 & 82 & .29 & .14 & .48 \\
\hline$e_{5} \mathrm{BCl}_{3}+15 \mathrm{H}_{2} / \mathrm{Ar}$ & .40 & 75 & .30 & .10 & .34 \\
\hline $\mathrm{f}_{1}\left(\mathrm{CH}_{3}\right)_{4} \mathrm{St}+5 \mathrm{H}_{2} / \mathrm{Ar}$ & .28 & 78 & .22 & .08 & .36 \\
\hline
\end{tabular}

aMeasured by a quadrupole mass spectrometer sampling the plasma at different locations (Avni (1984) and Avni et al. (1983b)).

BMicrocrystalline Si deposited on ATJ graphite, grounded, $P=2$ torr (data from Havens et al. (1976)).

${ }^{C} \mathrm{Si}_{3} \mathrm{~N}_{4}$ deposited on AISI 400 steel, floated, $\mathrm{P}=4$ torr (data from Ron et al. (1983)).

dPyrocarbon deposited on ATJ graphite, blased at $-100 \mathrm{~V}, P=7$ torr (data from Inspektor et al. (1981)).

e Boridation of AISI400 steel, grounded FeB, $P=3$ torr (data from Raveh et al. (1983)).

fsic deposited on Ti alloys, grounded, $P=4$ torr (data from Katz et al. (1980)).

gsee text and khait et al. (1980). 


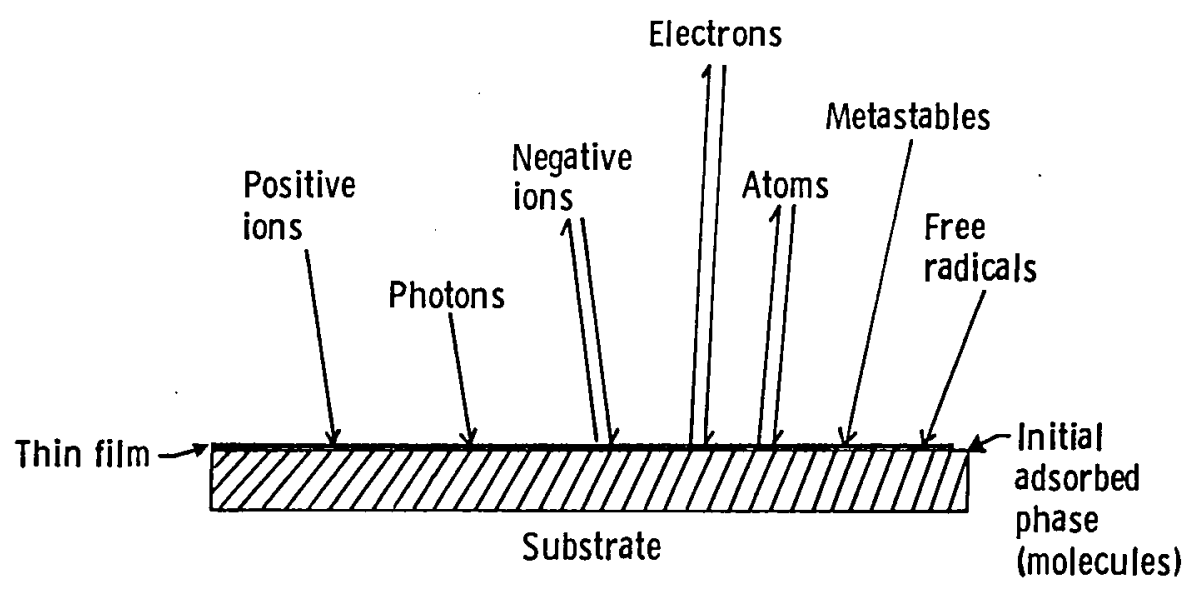

Figure 1.

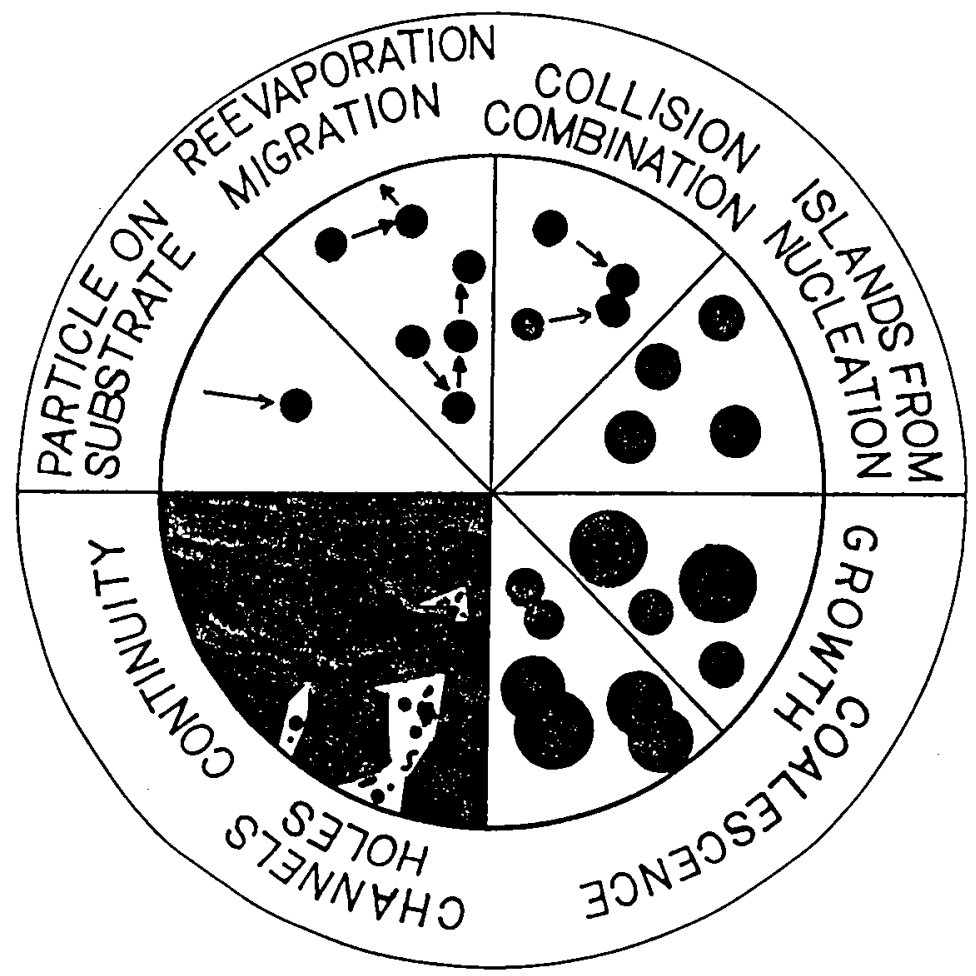

Figure 2. 


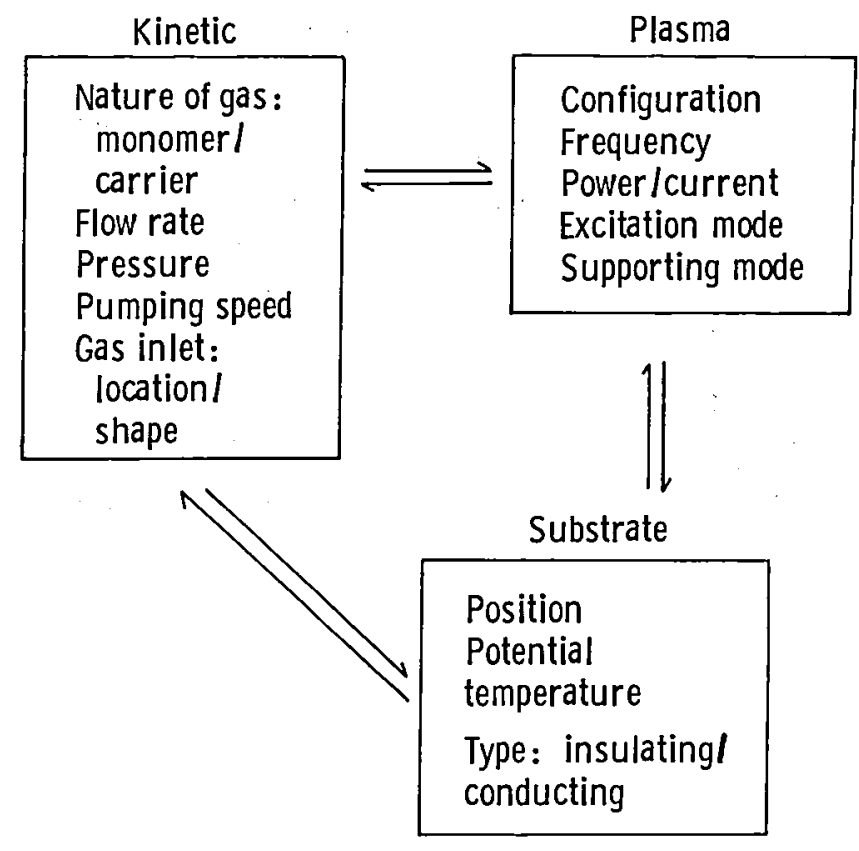

Figure 3.

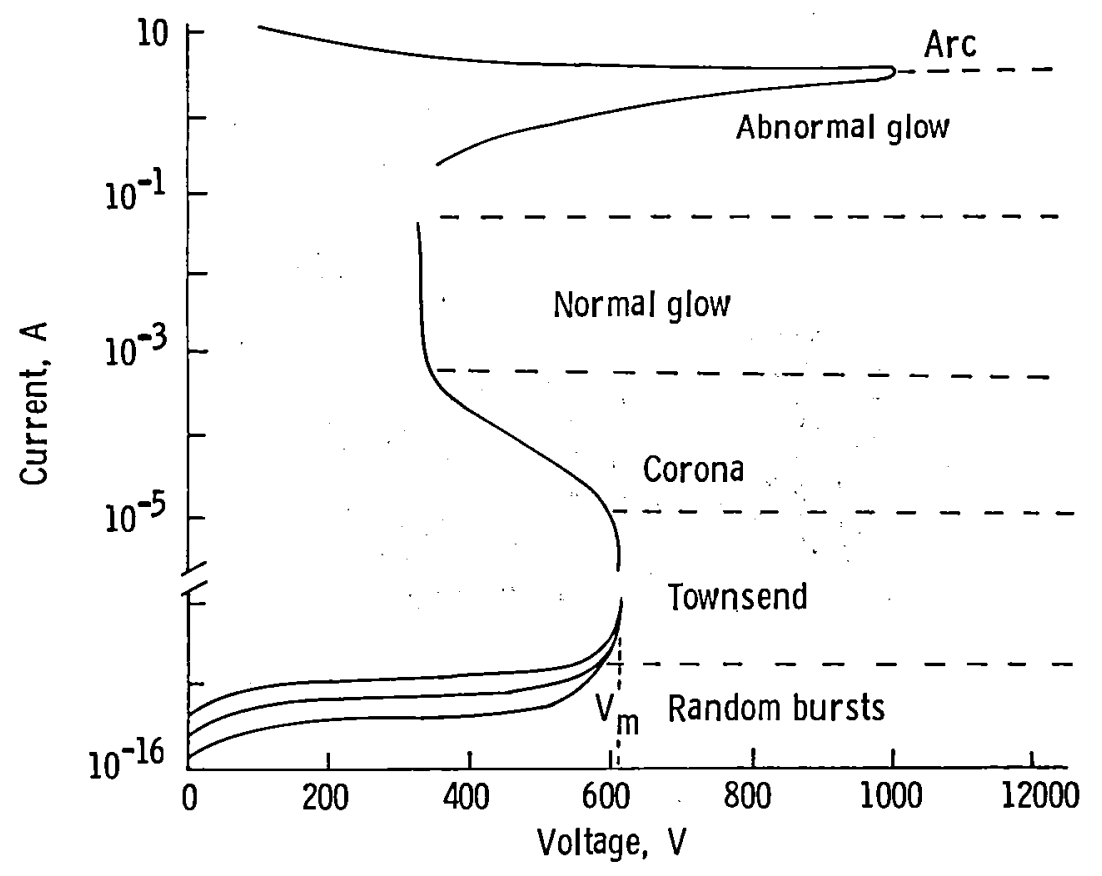

Figure 4. 


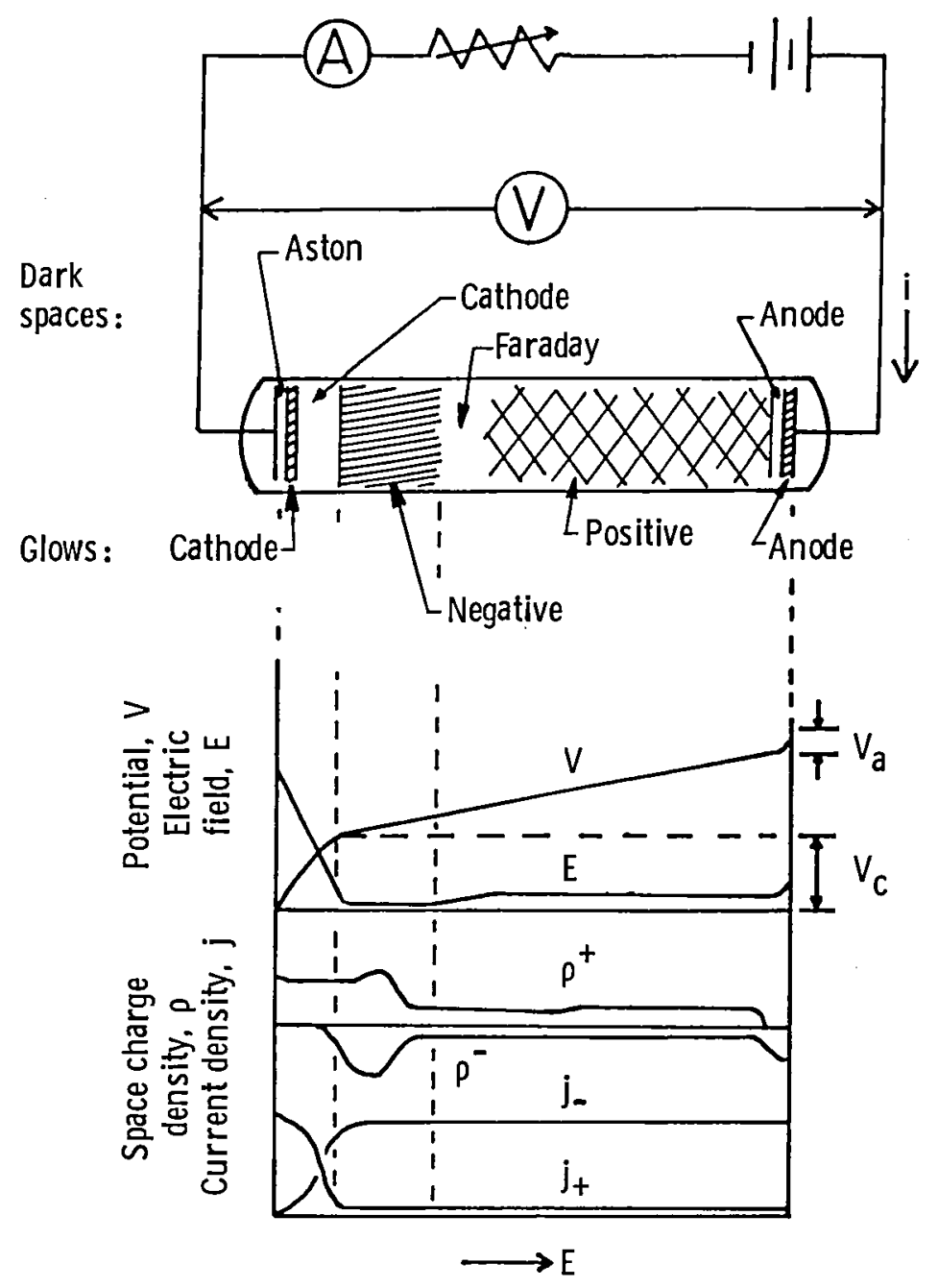

Figure 5.

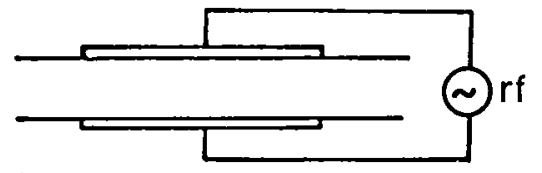

(a)
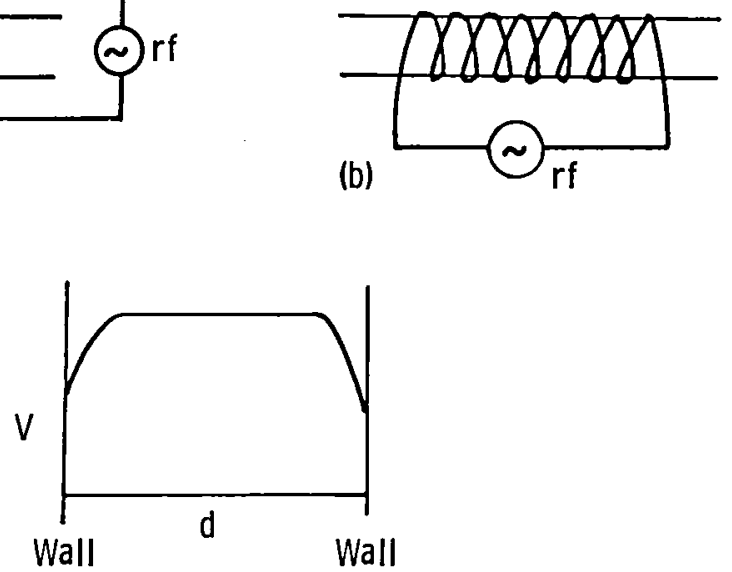

(c)

Figure 6. 


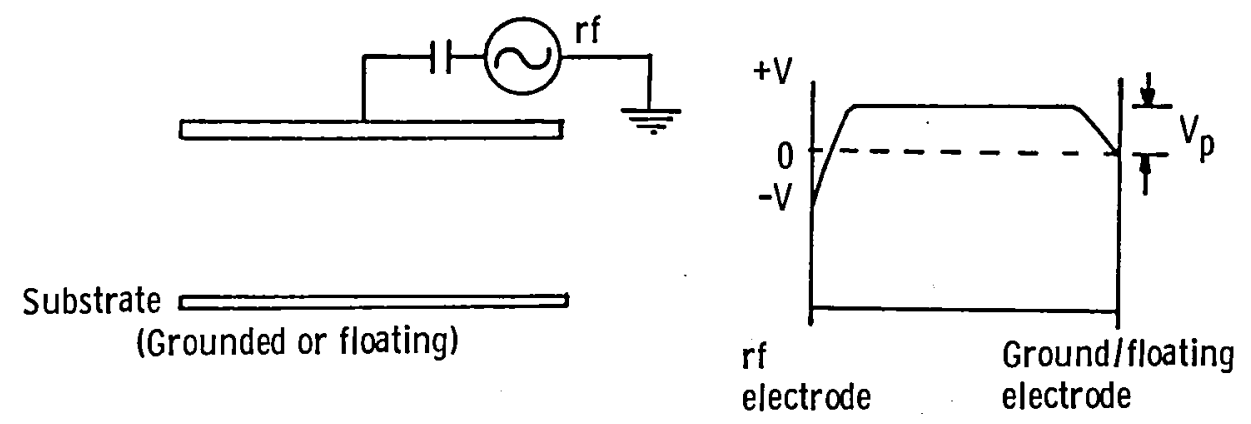

Figure 7.
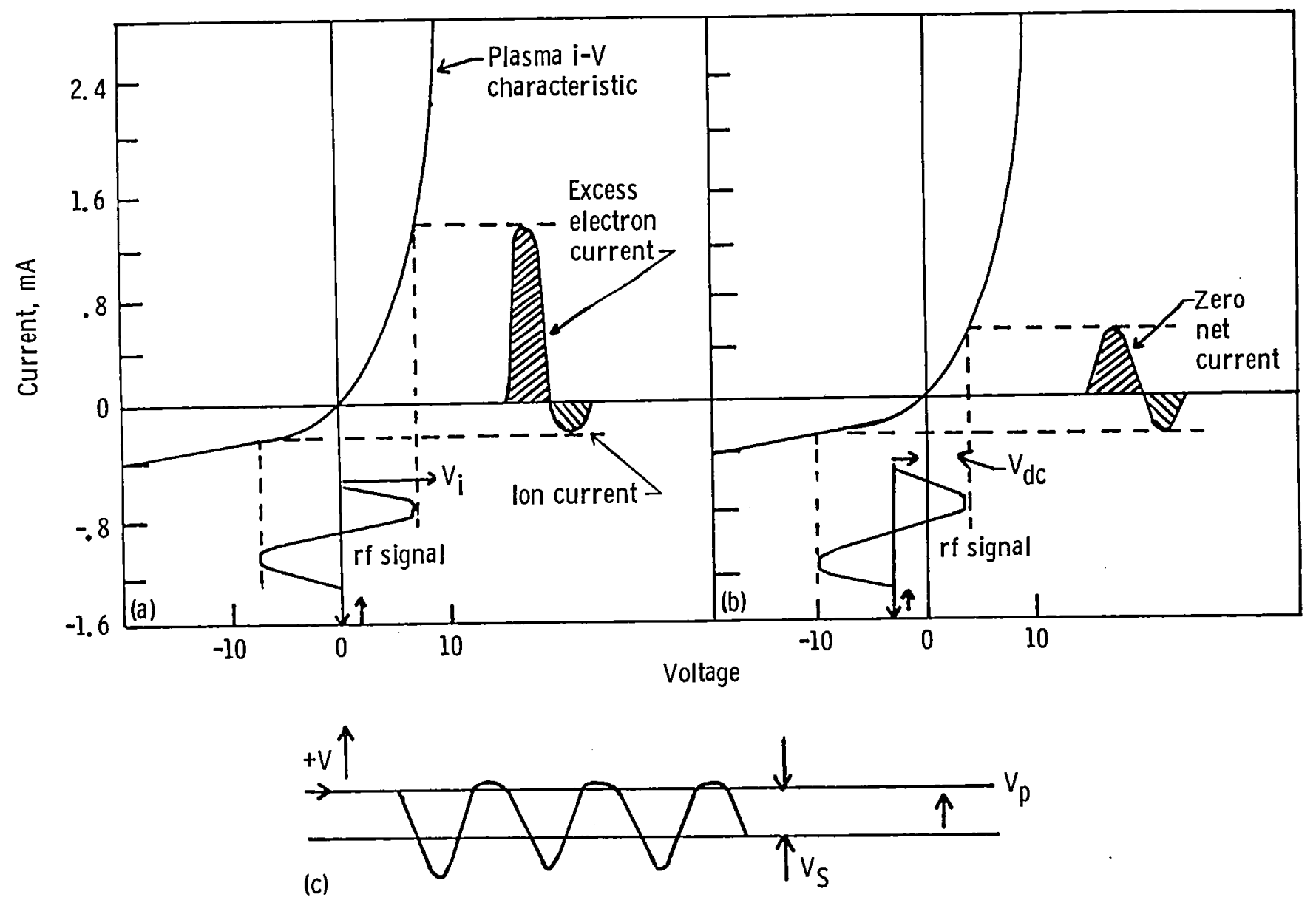

Figure 8. 


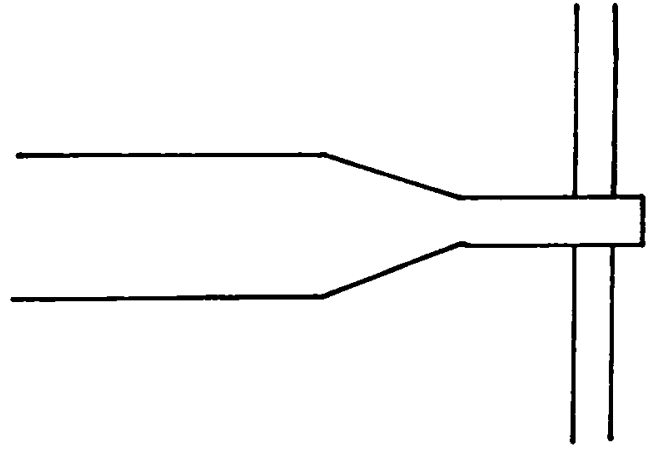

Wave guide

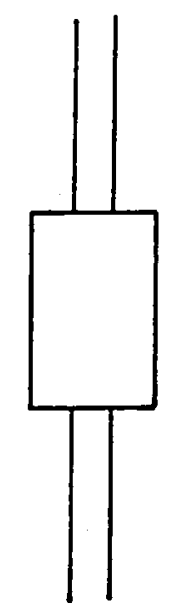

Resonant cavity

Figure 9.

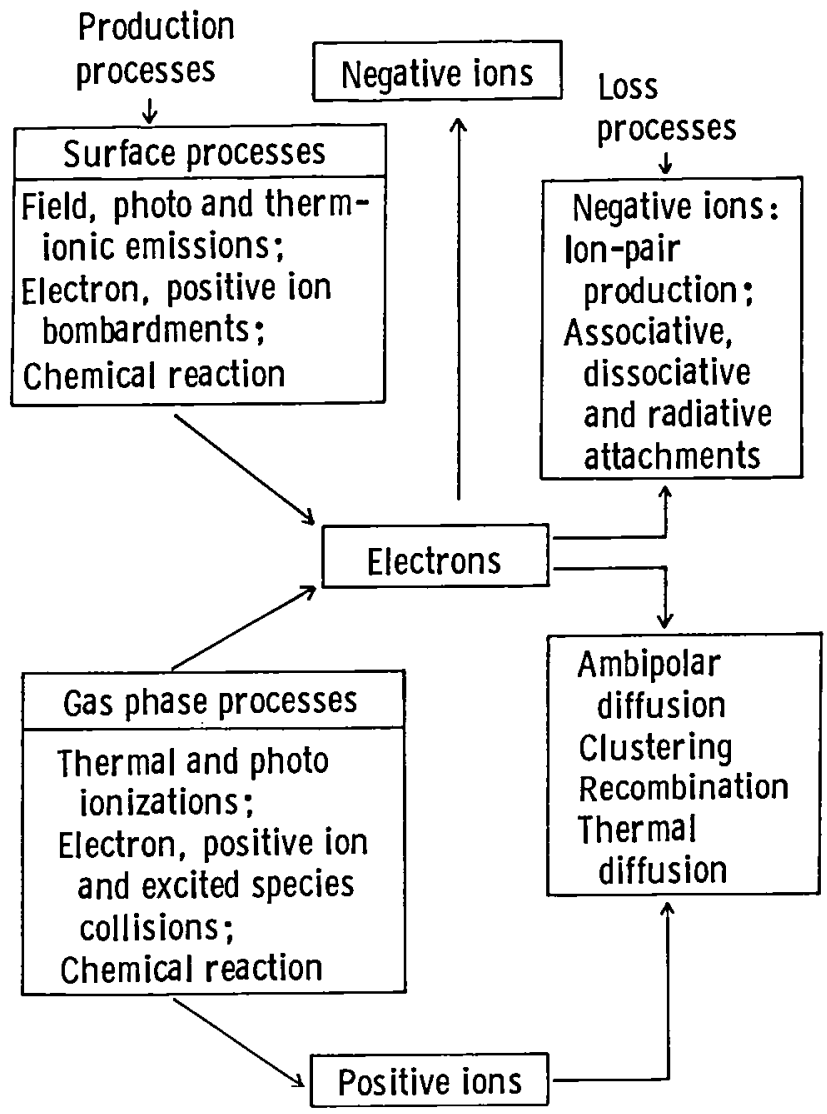

Figure 10. 


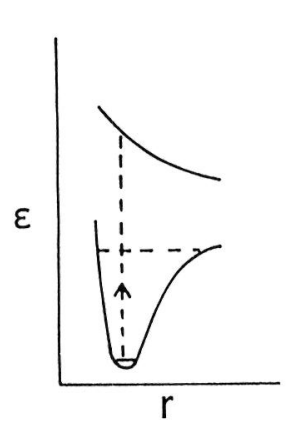

(a)

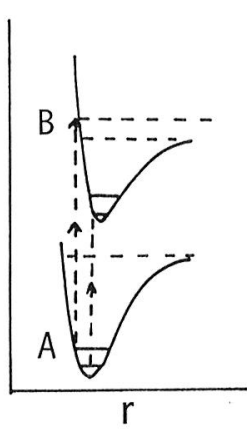

(b)

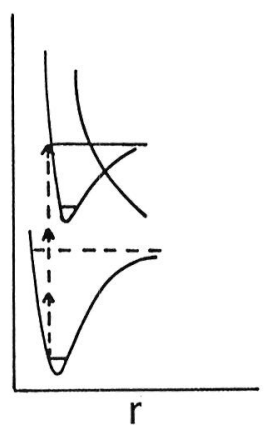

(c)

Figure 11.
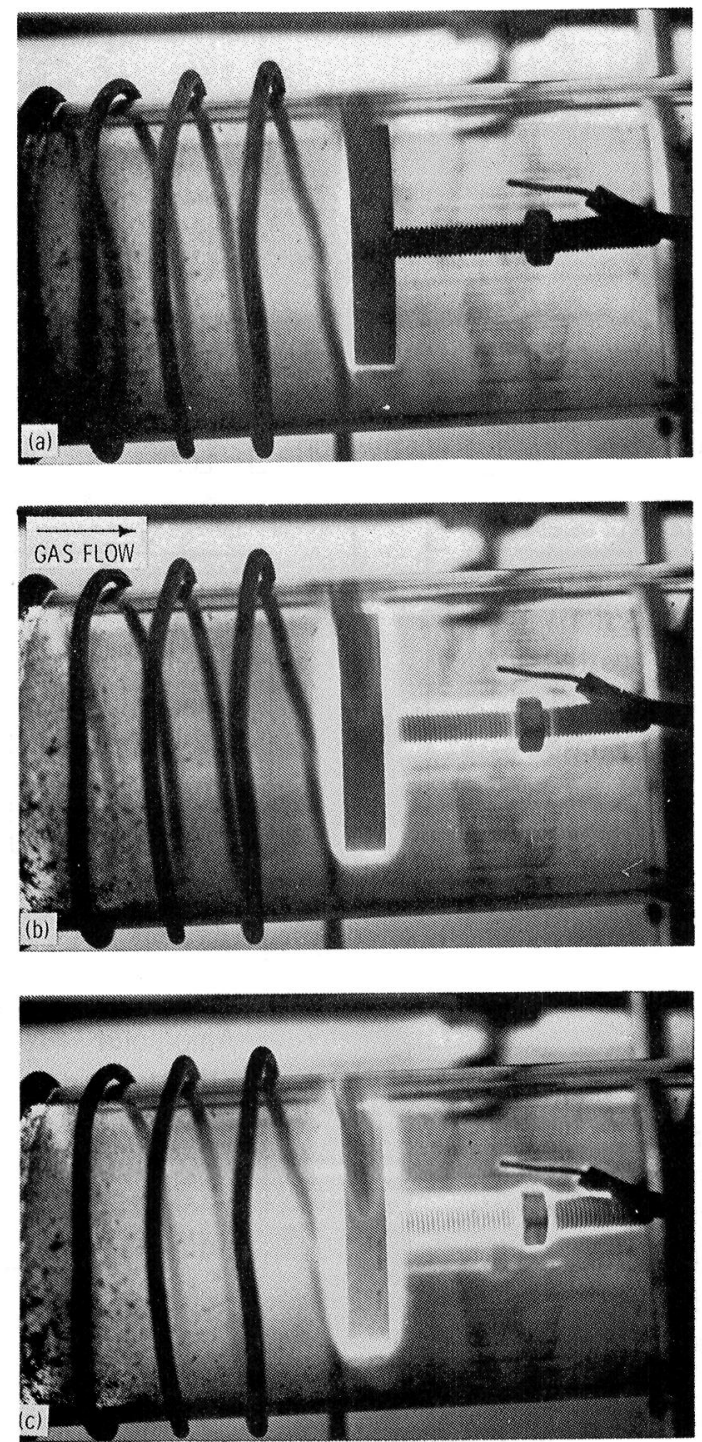

Figure 12. 


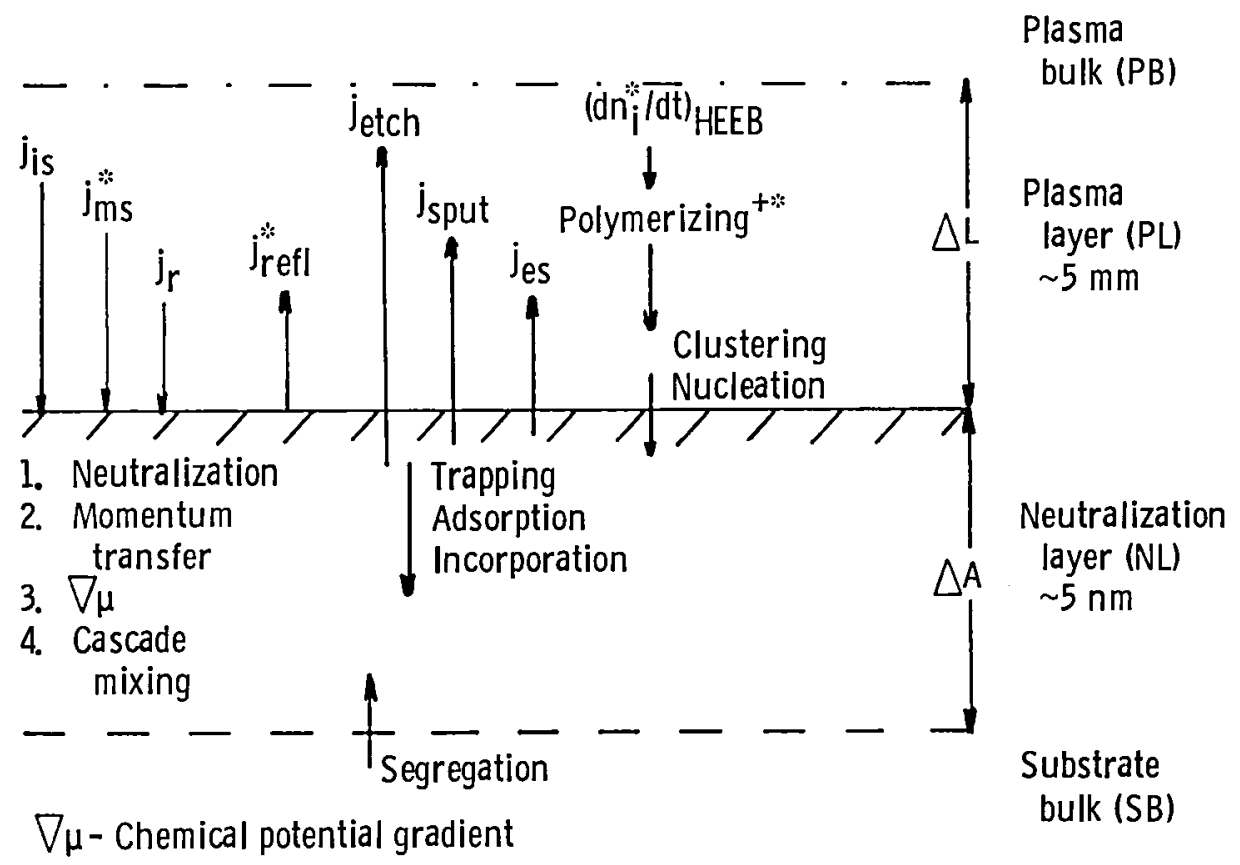

Figure 13.

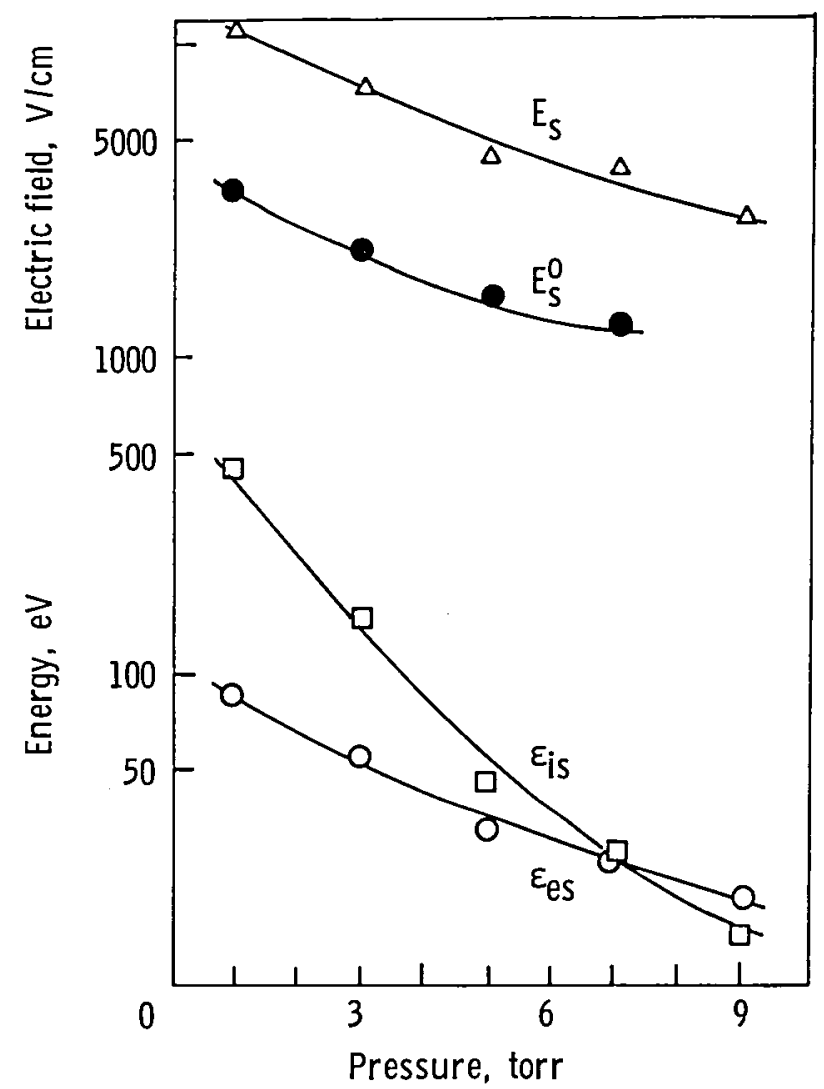

Figure 14. 


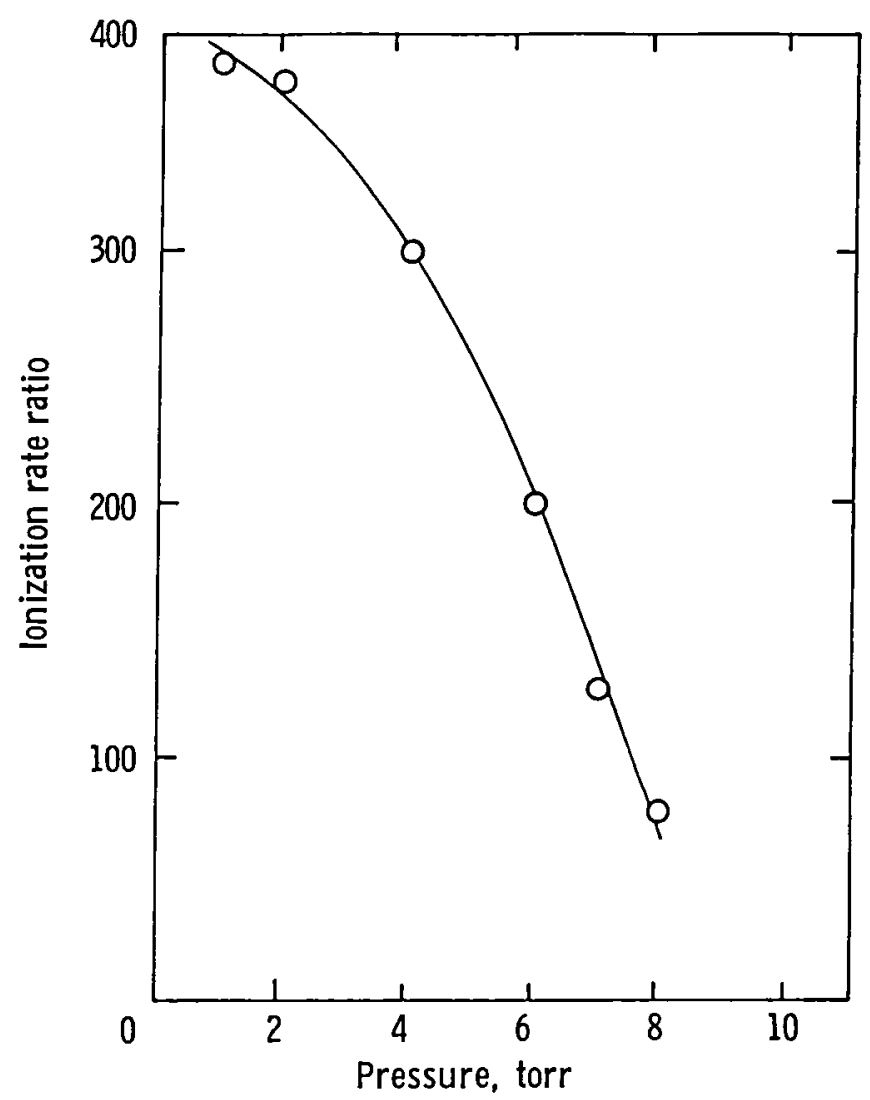

Figure 15. 


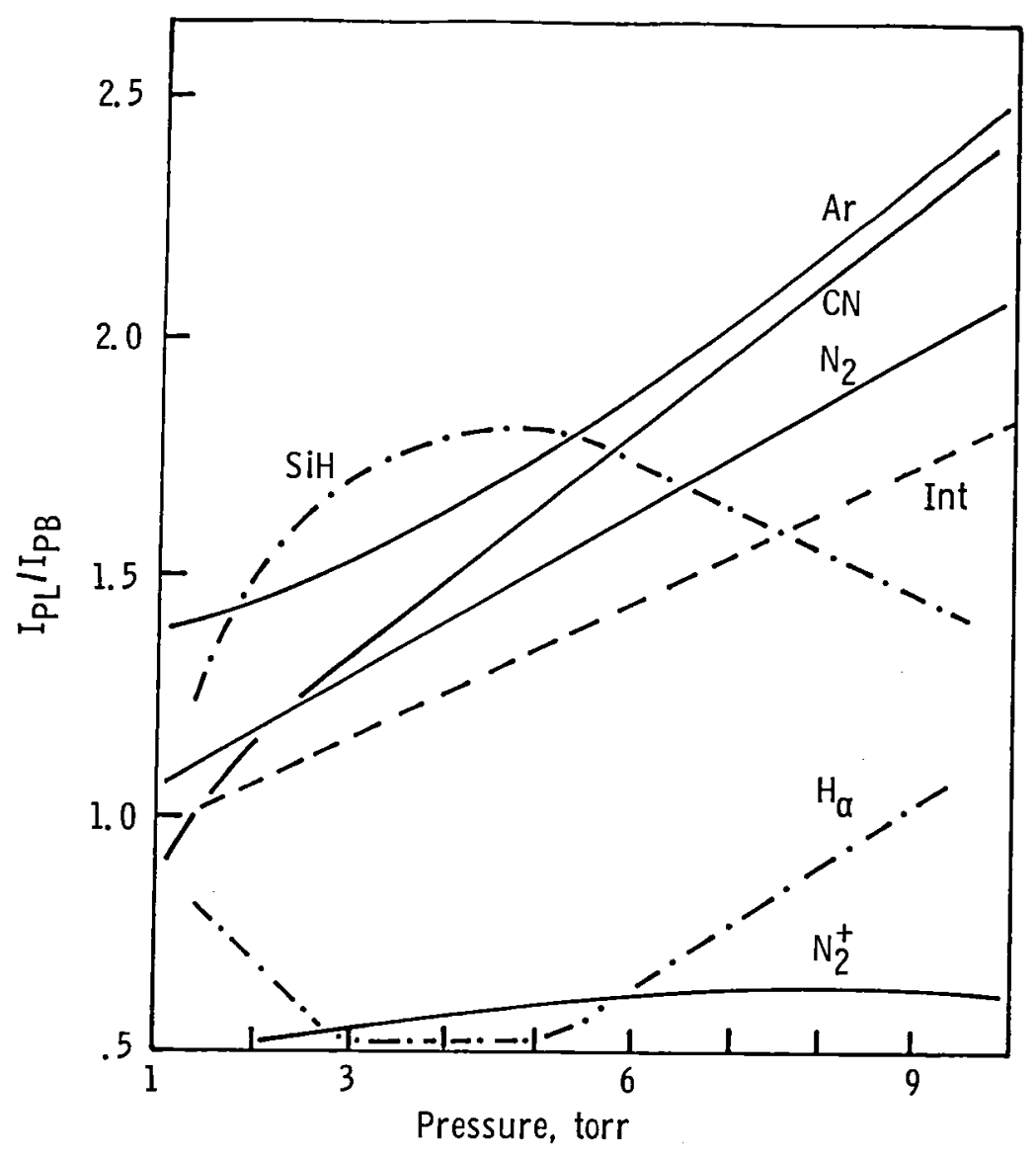

Figure 16. 


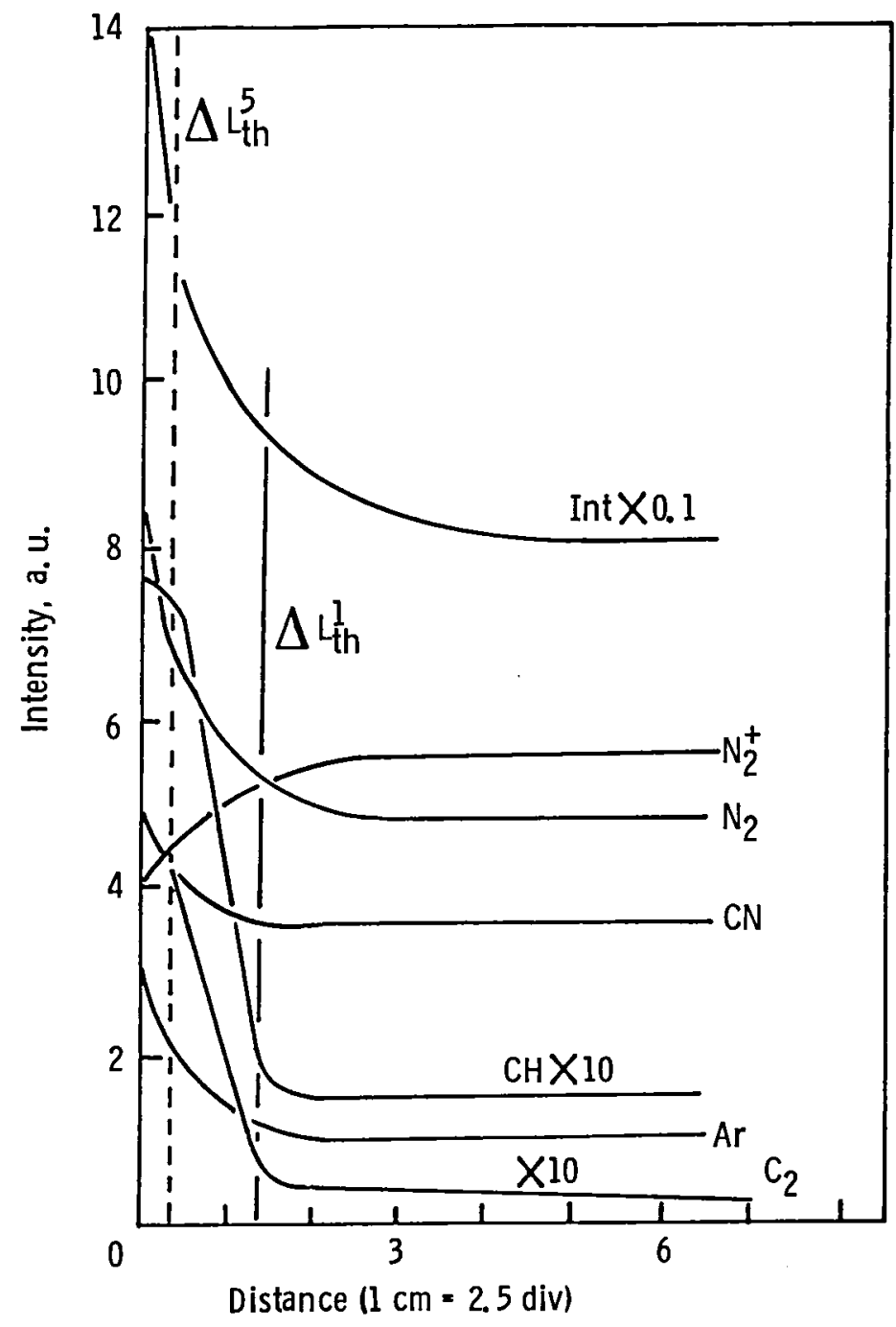

Figure 17.

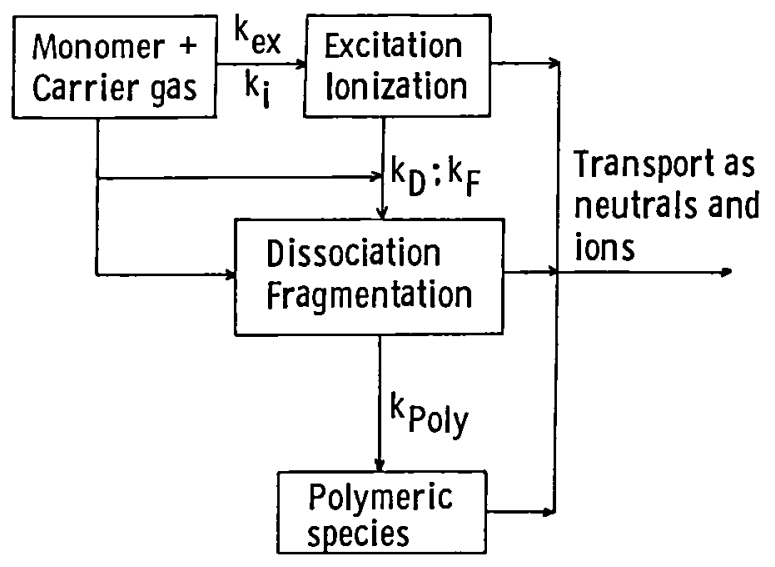

Figure 18. 


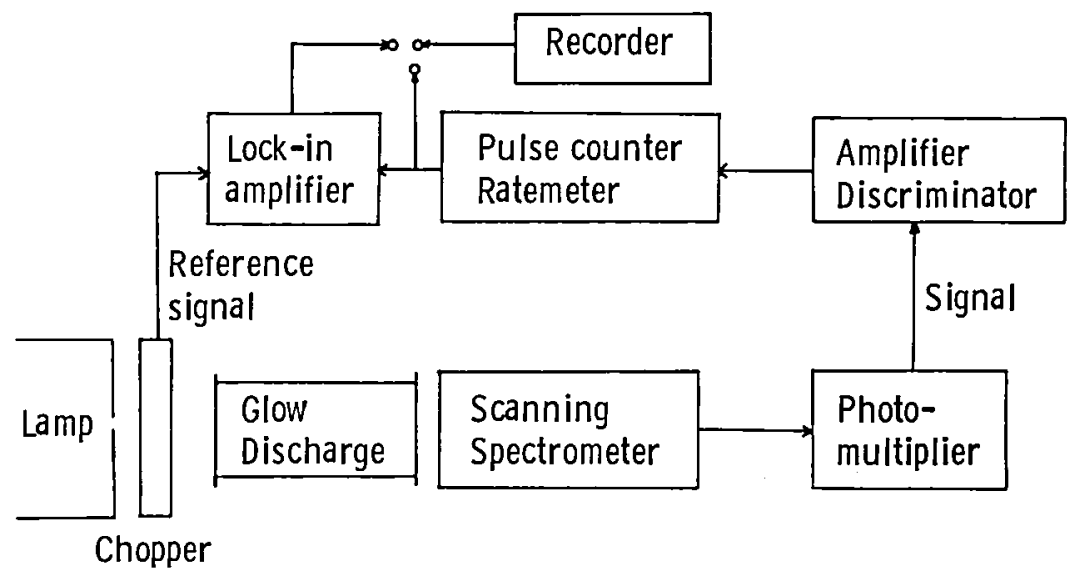

Figure 19.

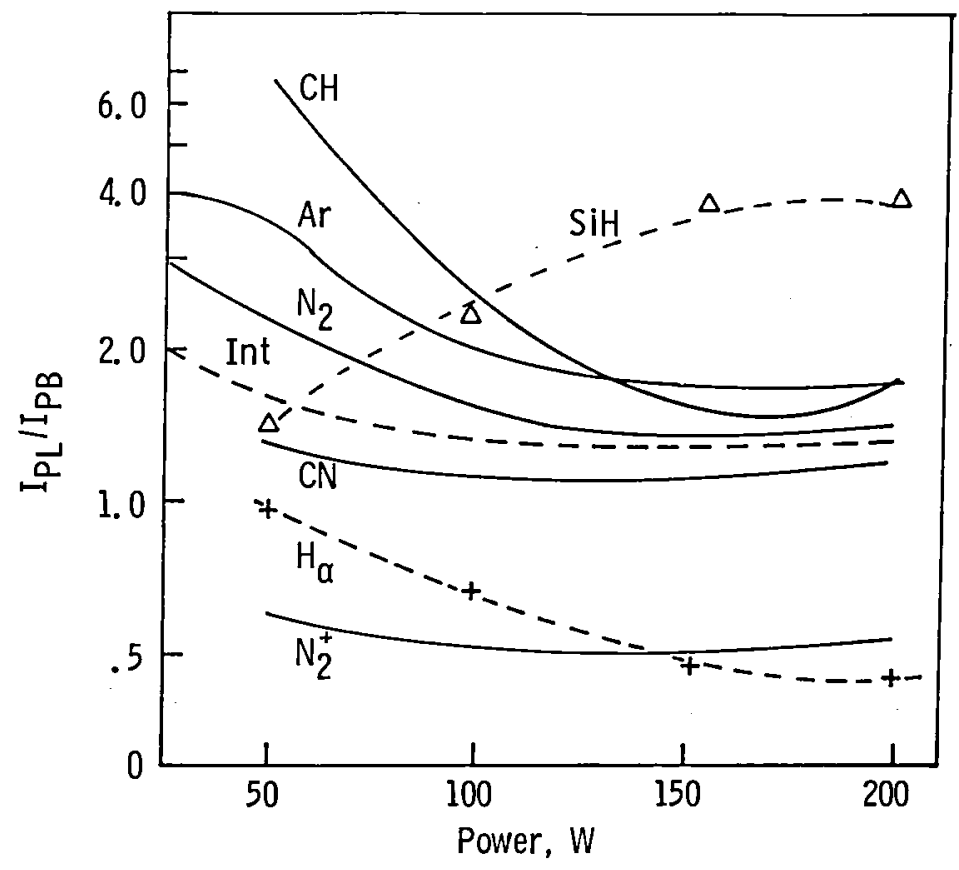

Figure 20. 


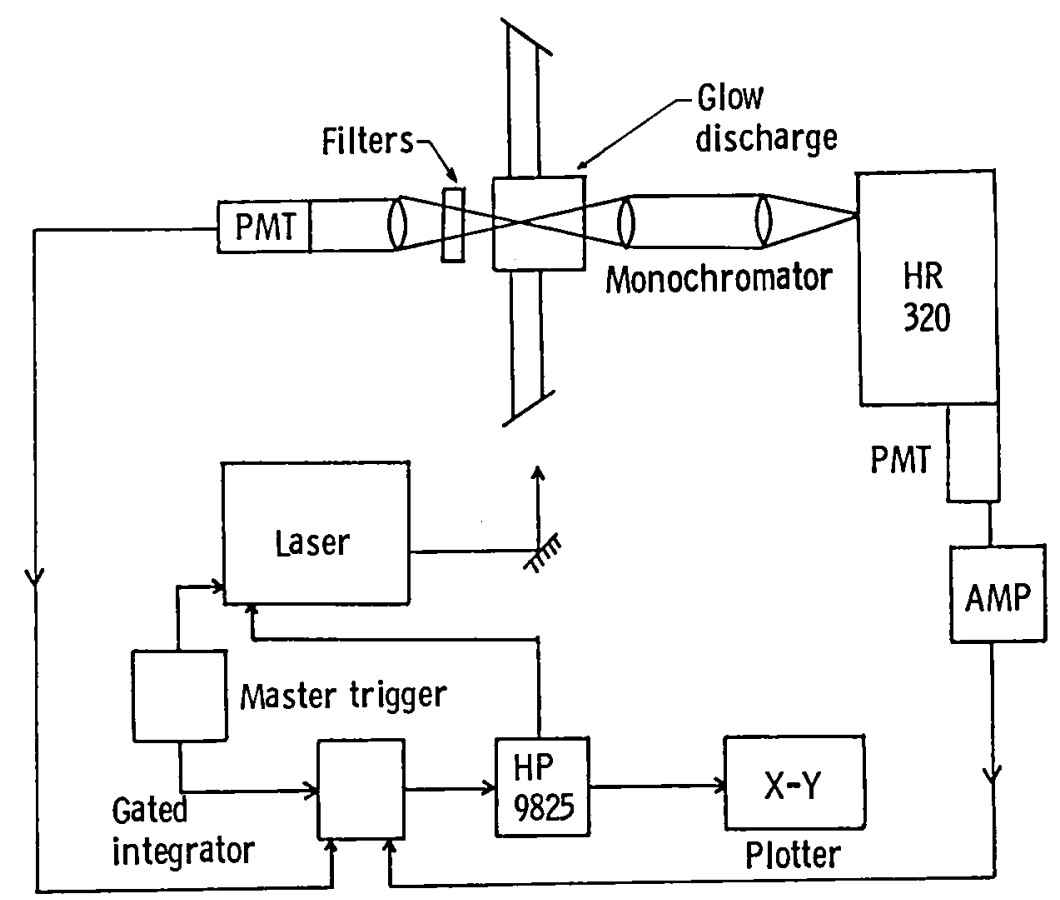

Figure 21.

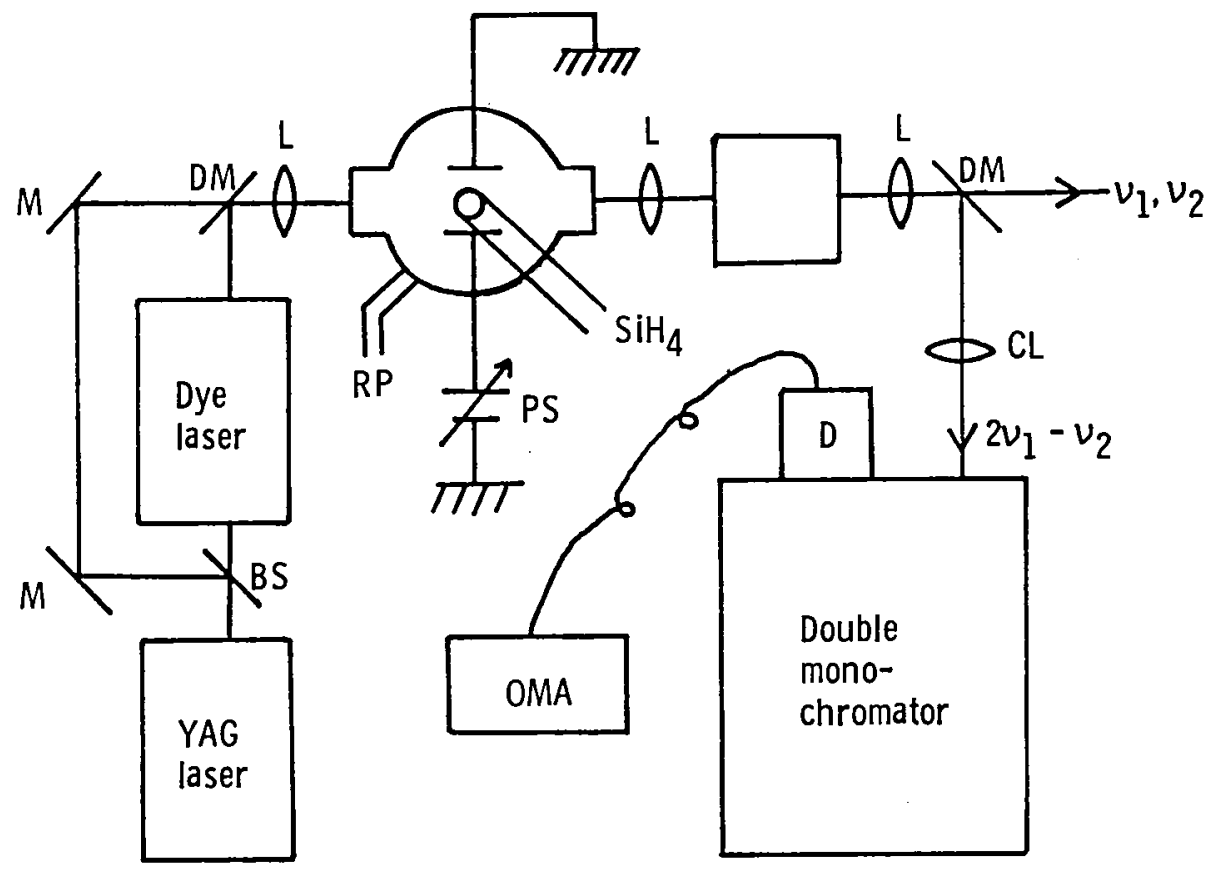

Figure 22. 


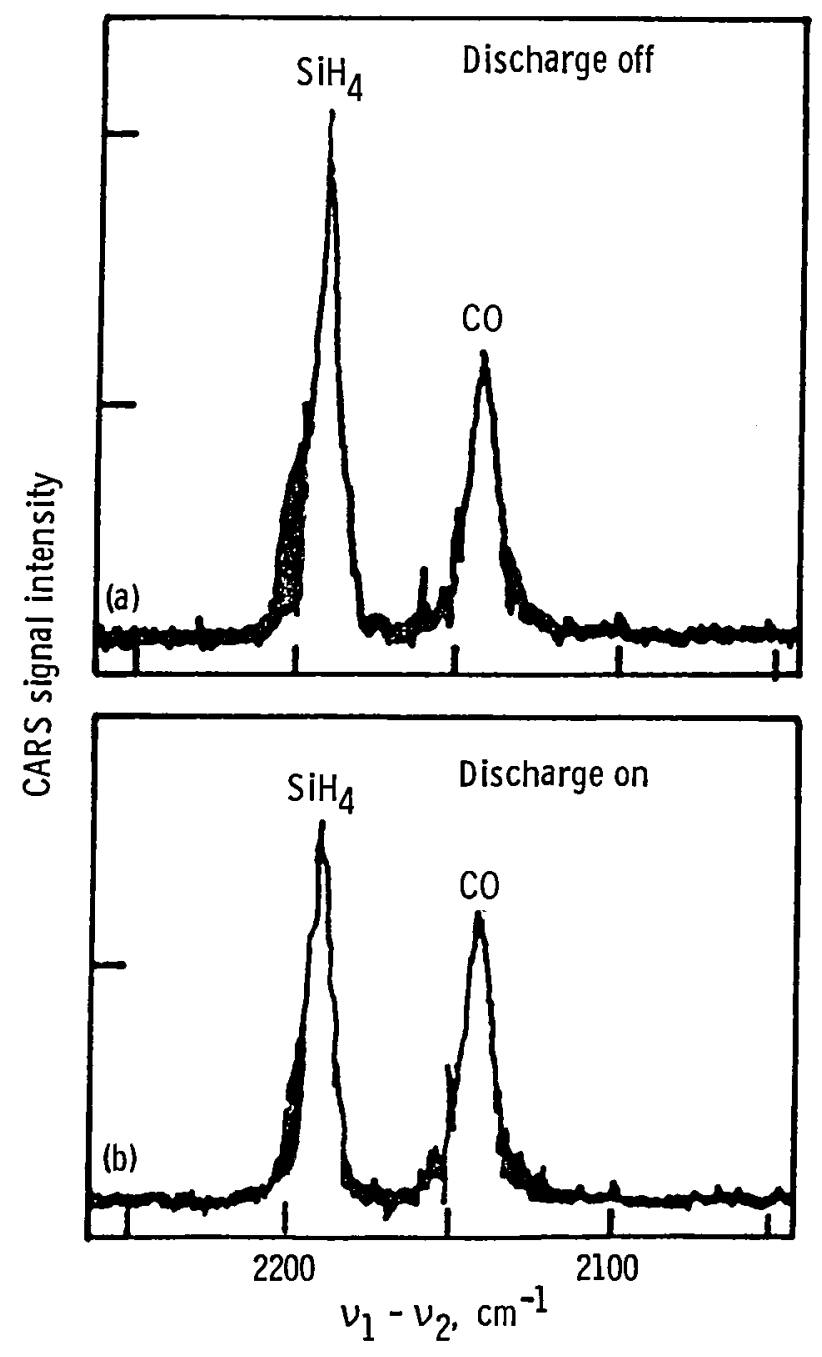

Figure 23.

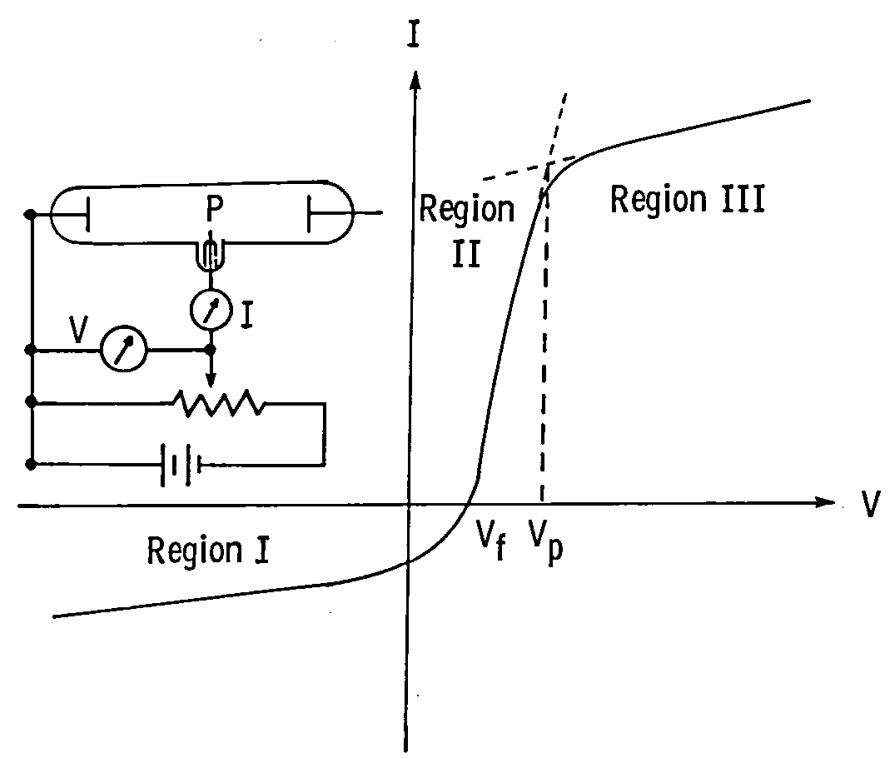

Figure 24. 


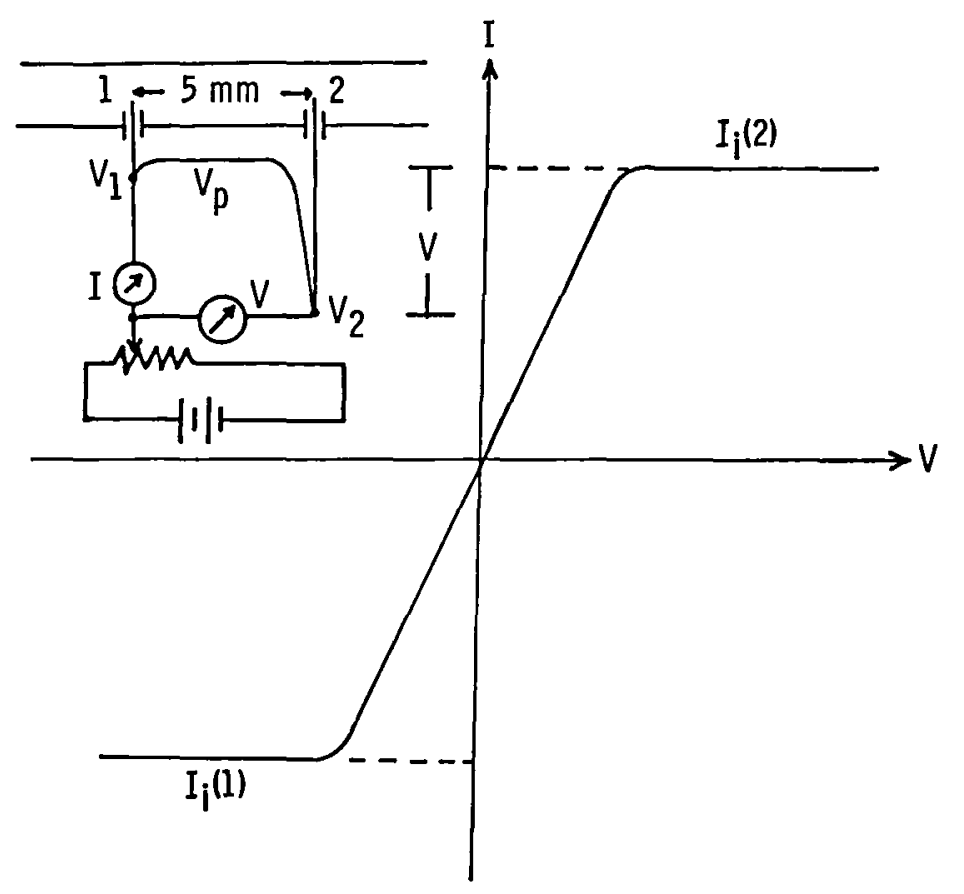

Figure 25.

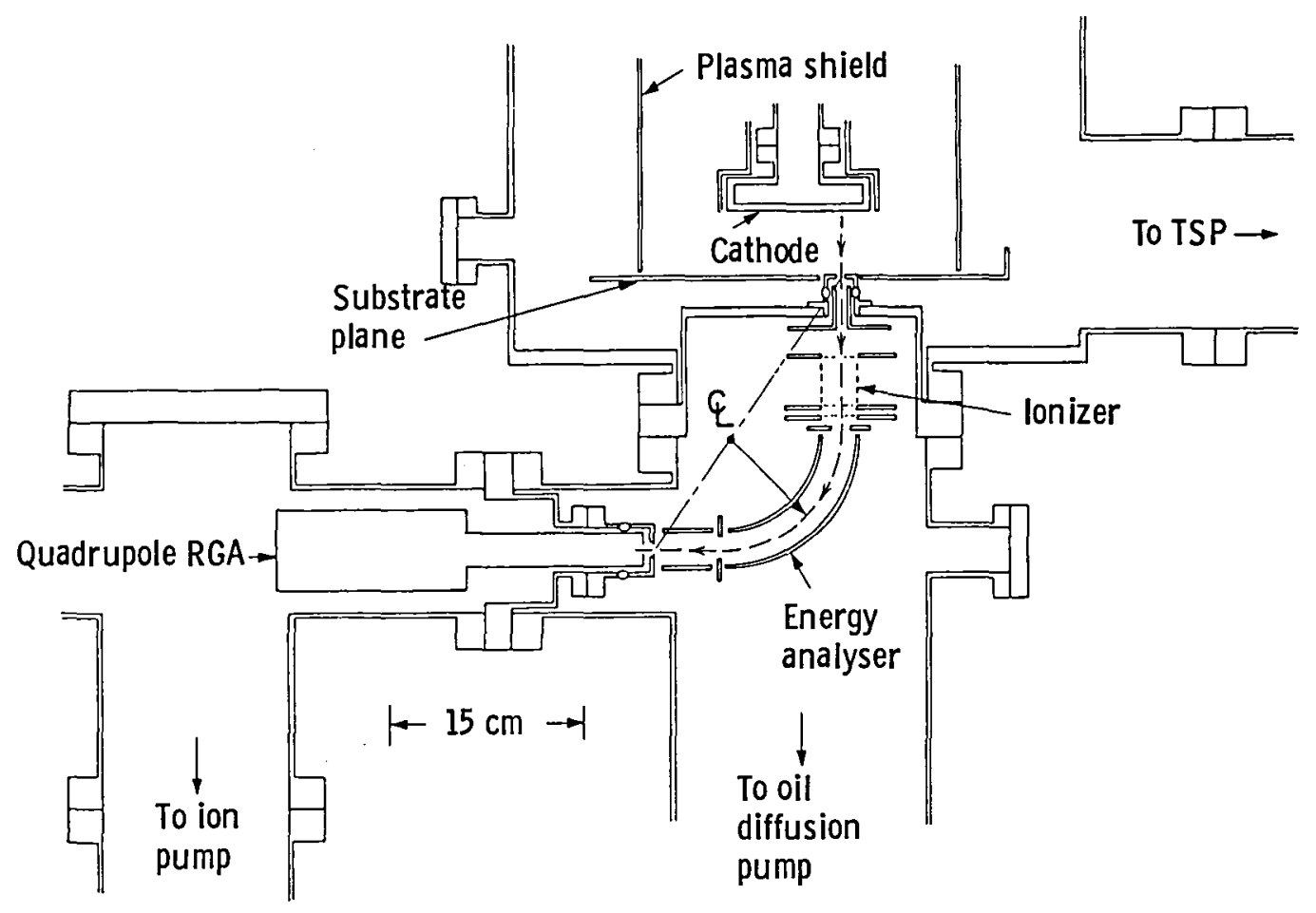

Figure 26. 


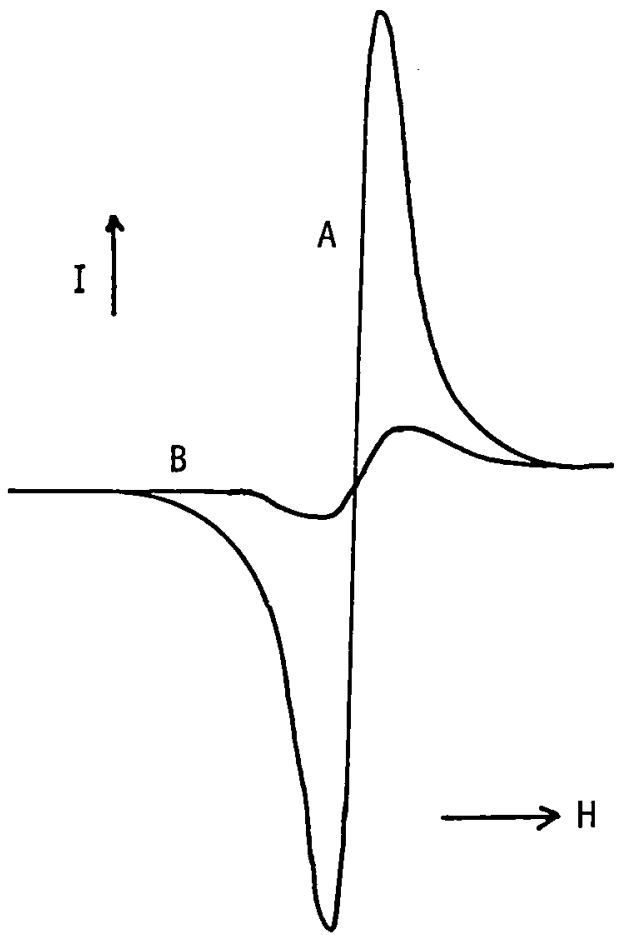

Figure 27. 


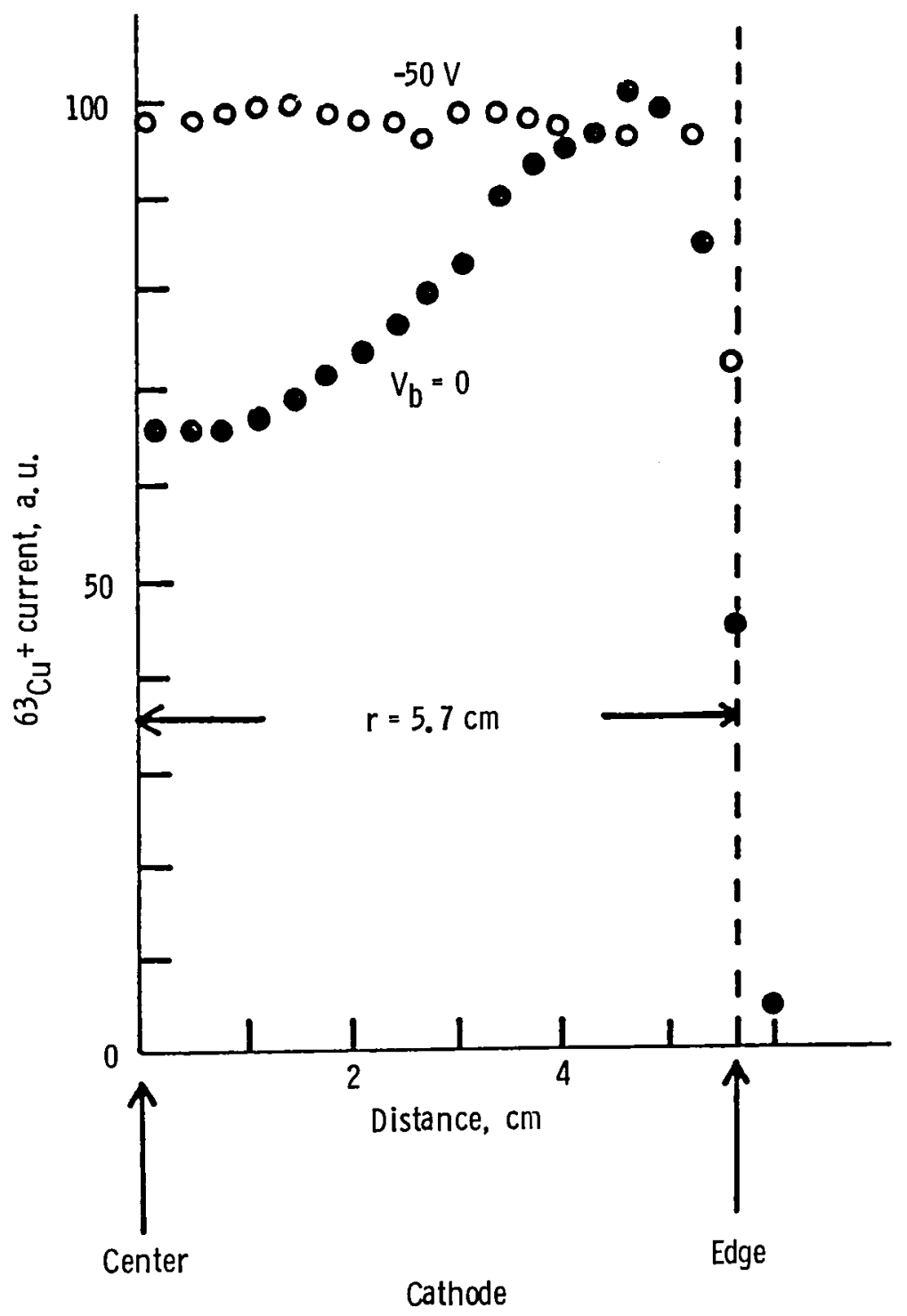

Figure 28. 


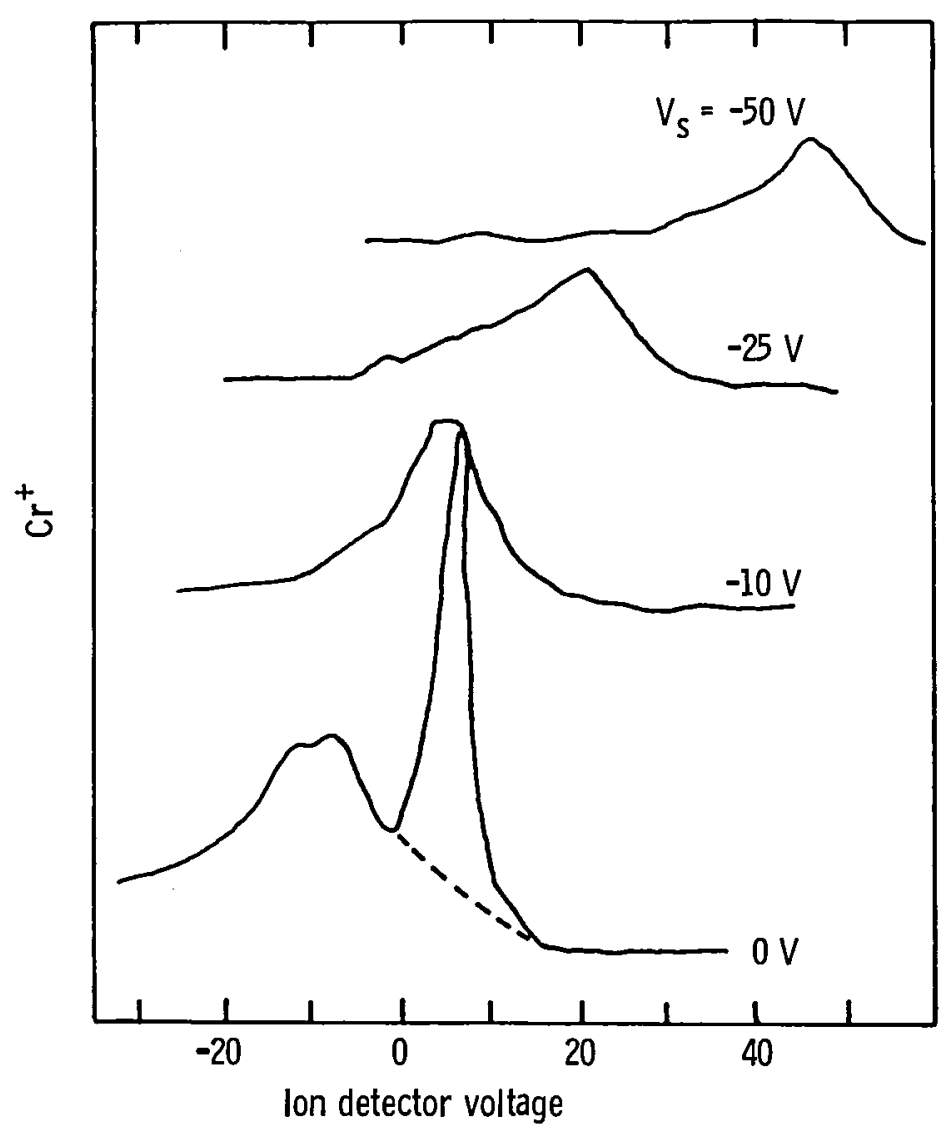

Figure 29.

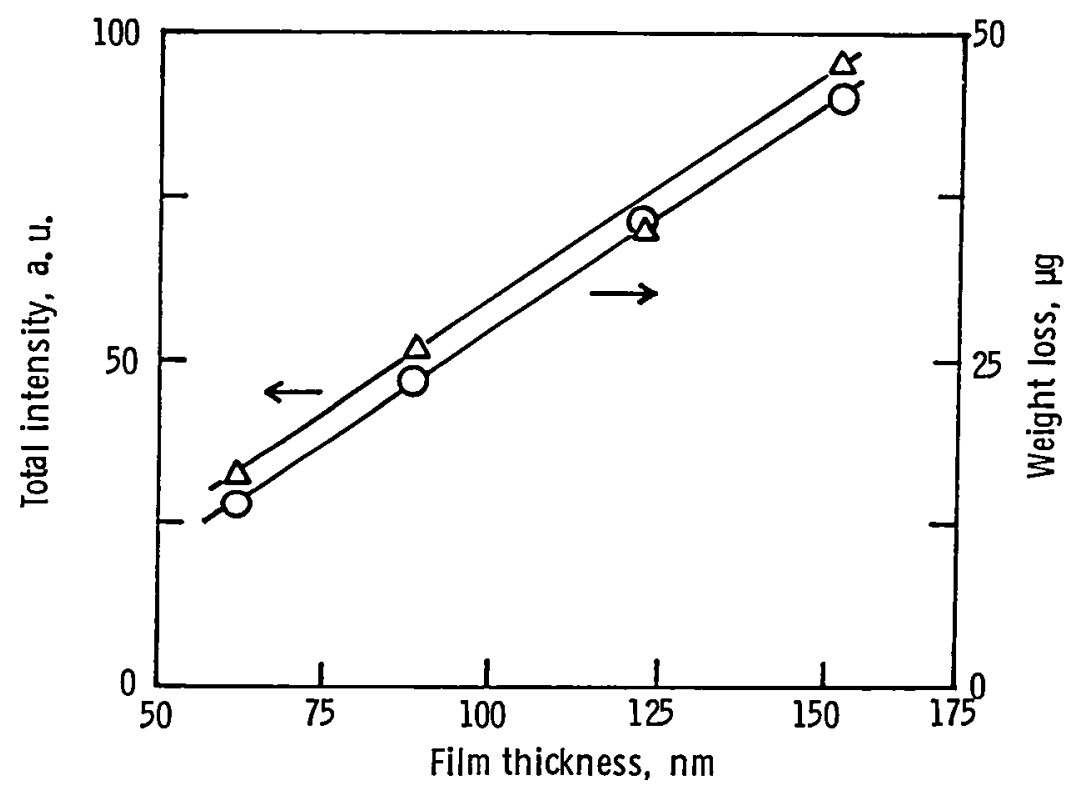

Figure 30. 


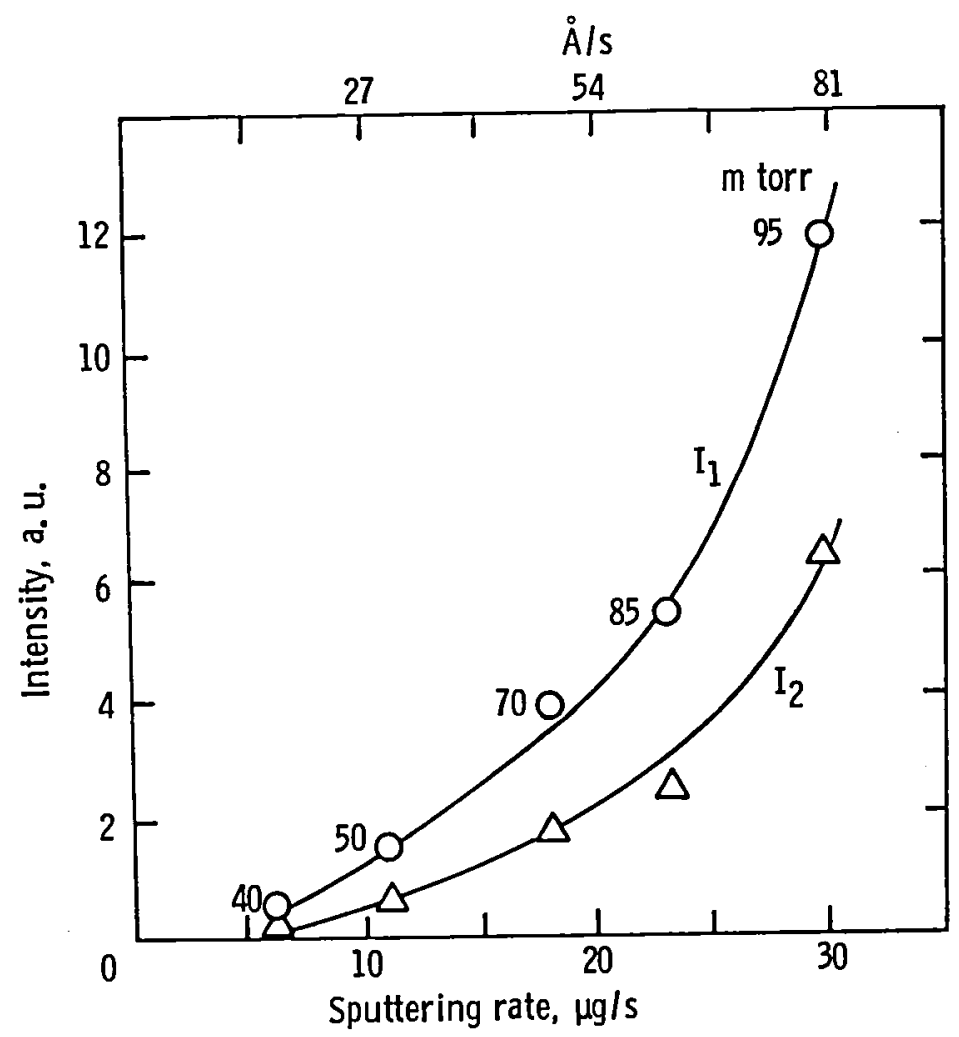

Figure 31.

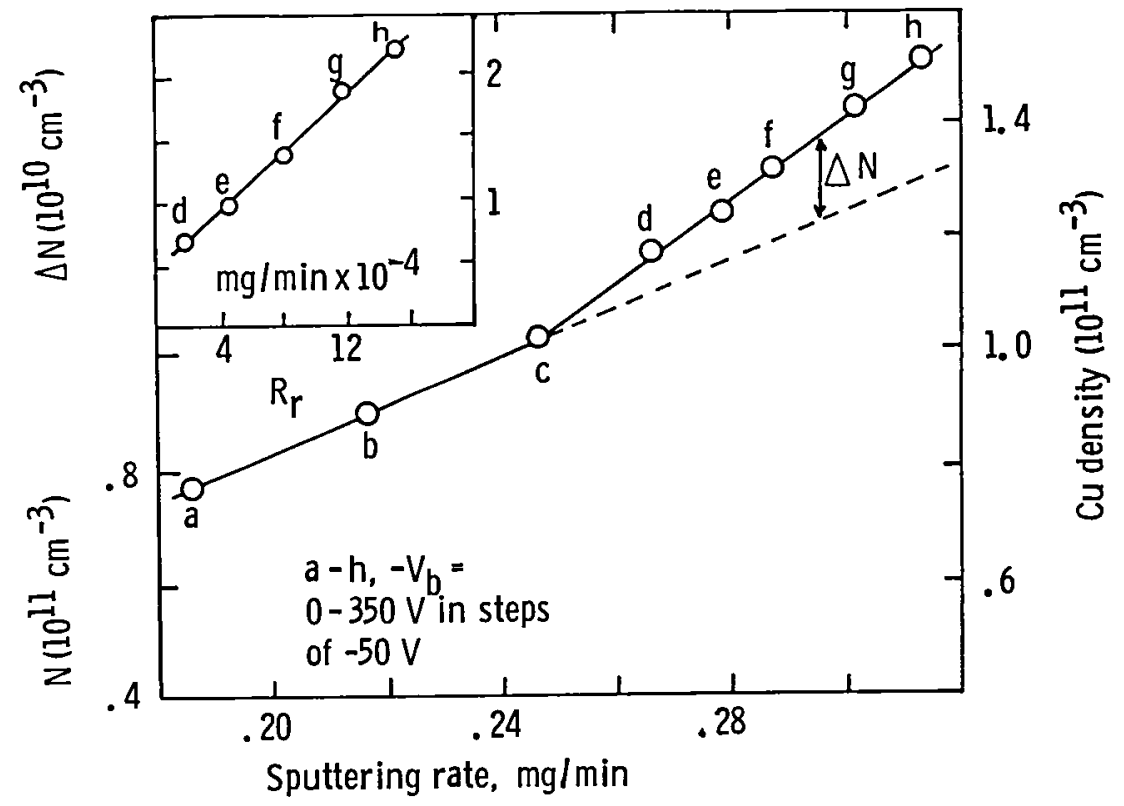

Figure 32. 


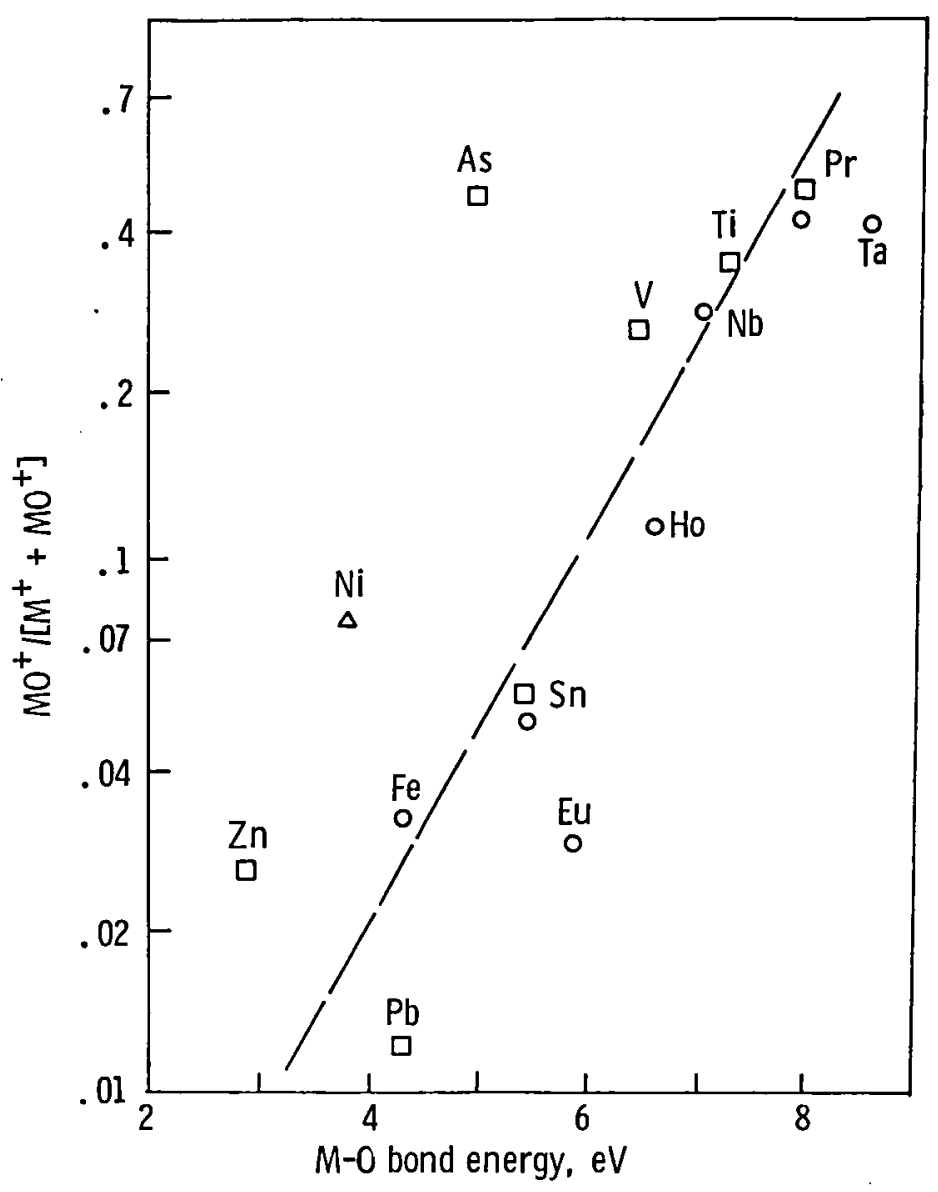

Figure 33. 


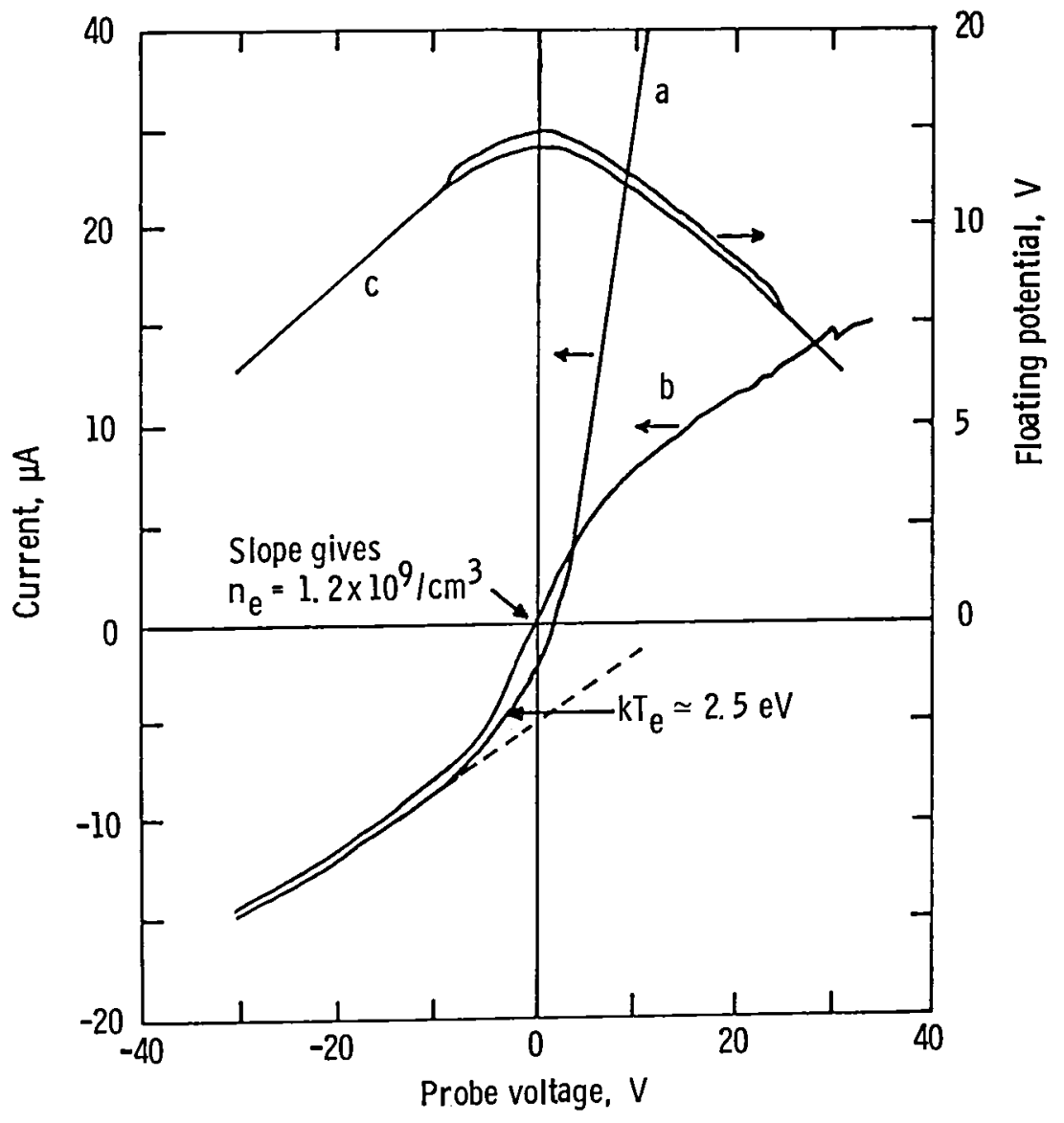

Figure 34.

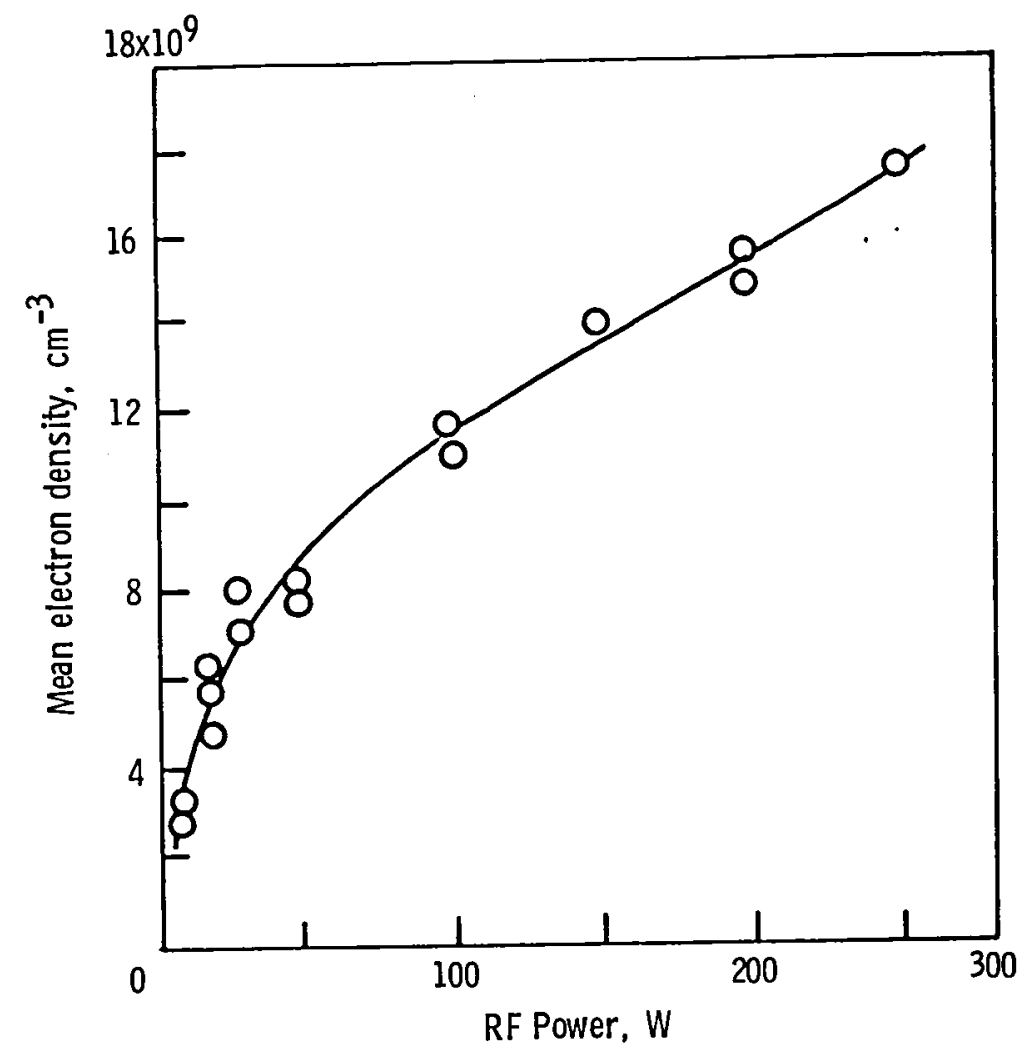

Figure 35. 


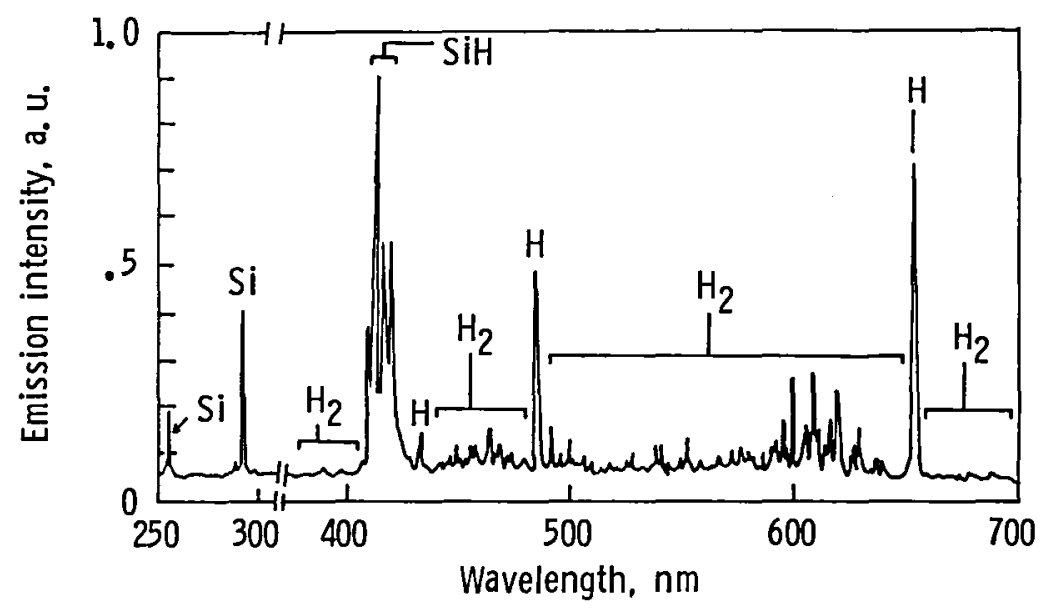

Figure 36.

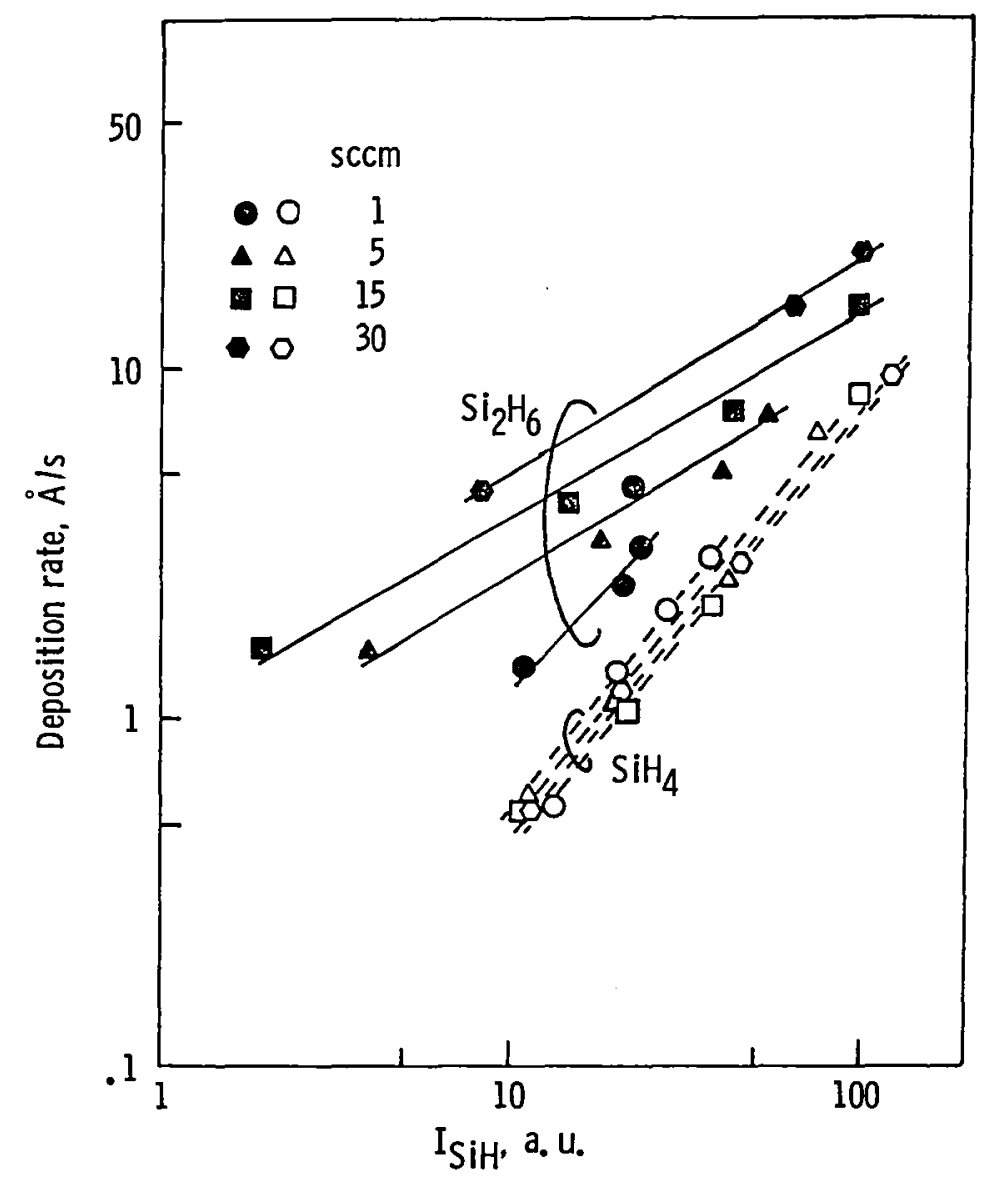

Figure 37. 


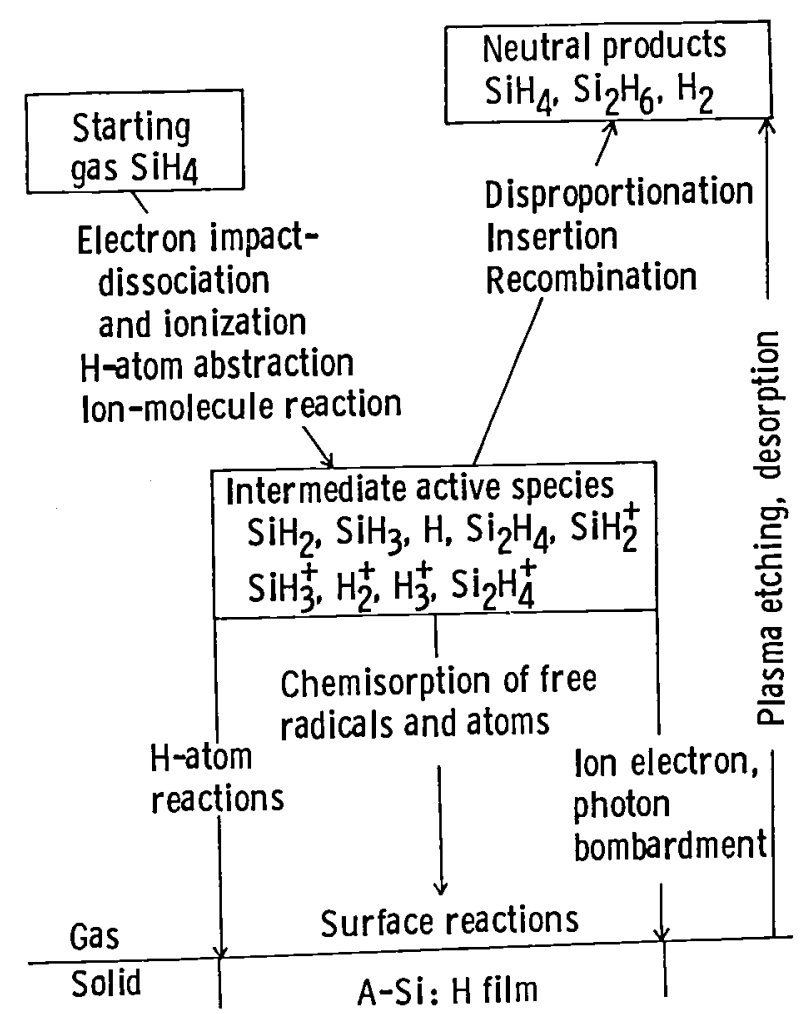

Figure 38.

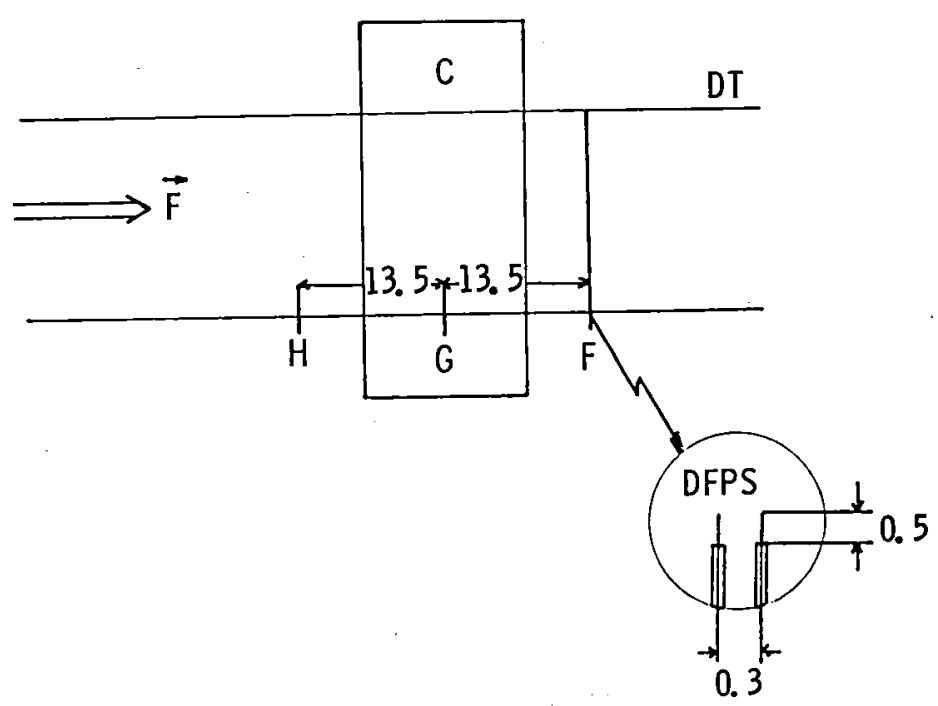

Figure 39. 


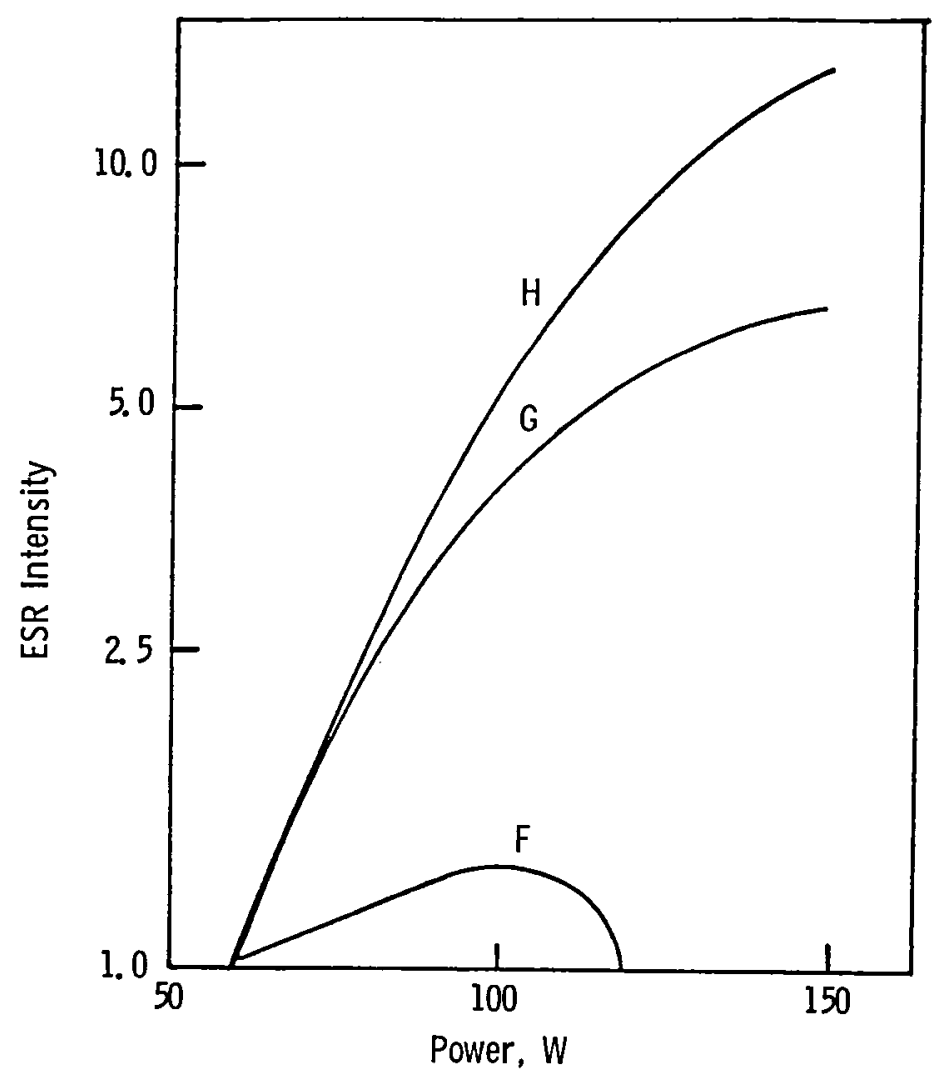

Figure 40.

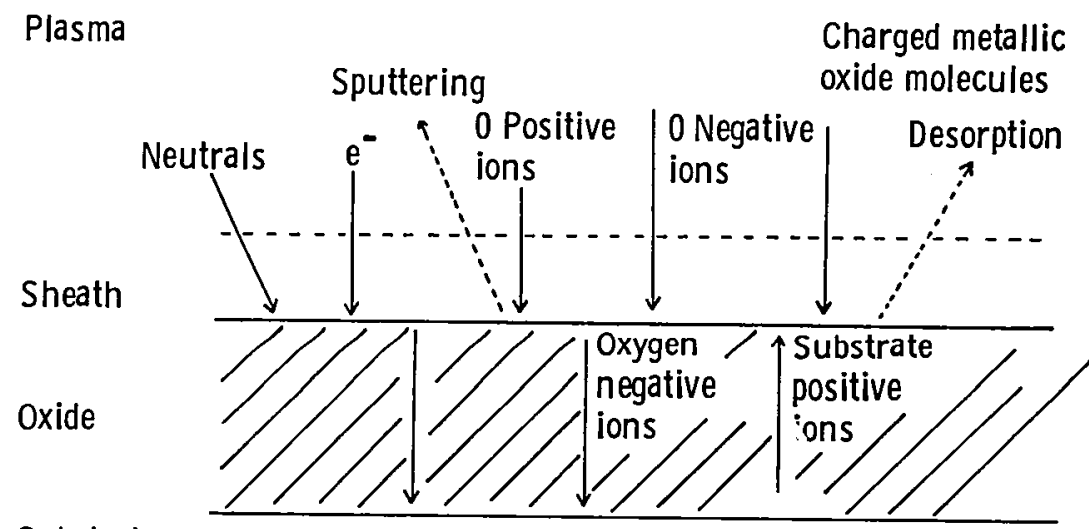

Substrate

Figure 41. 


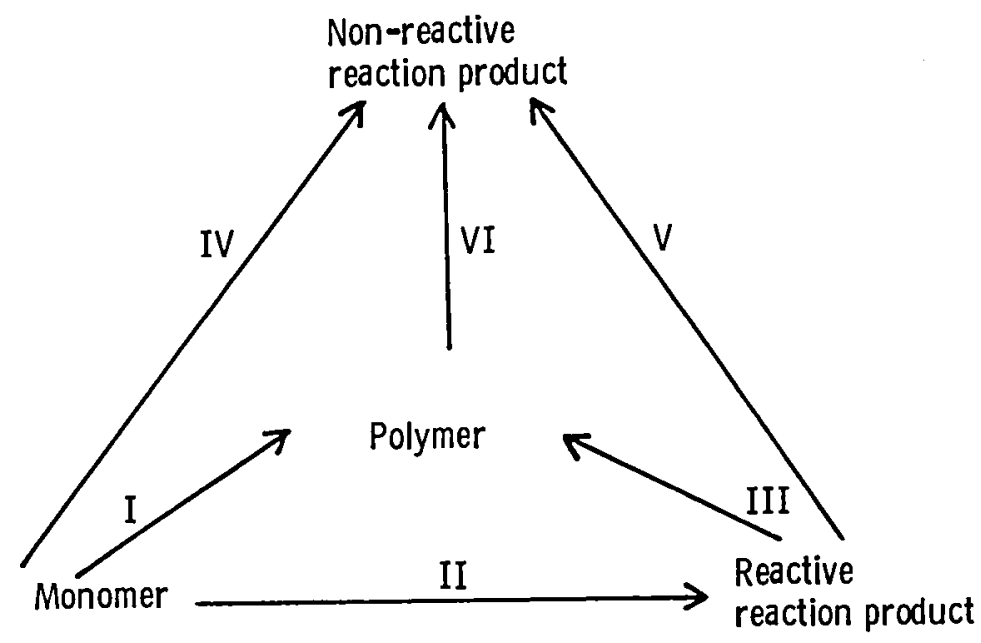

Figure 42.

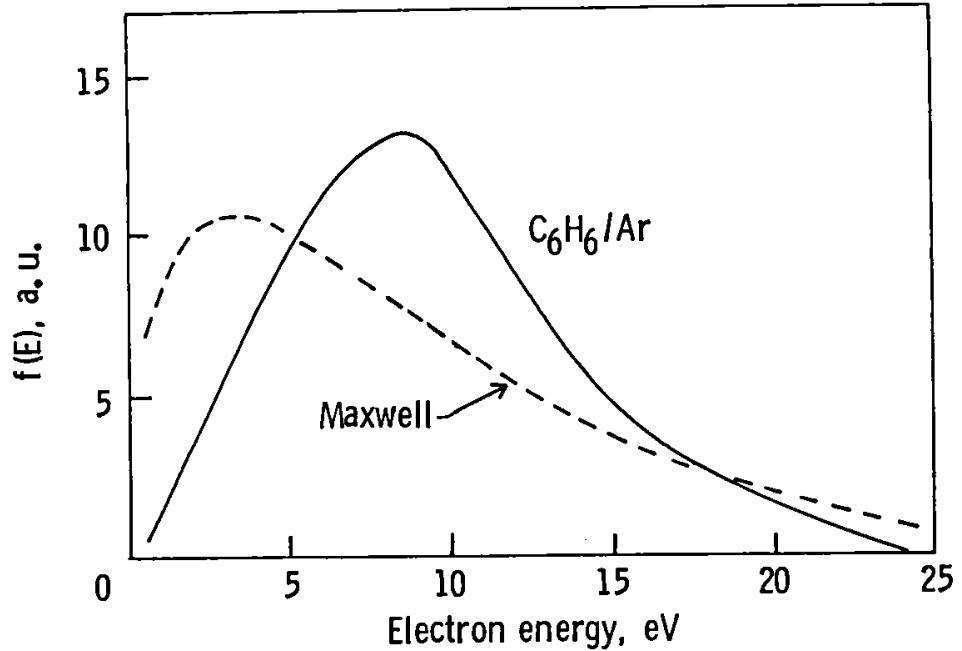

Figure 43. 


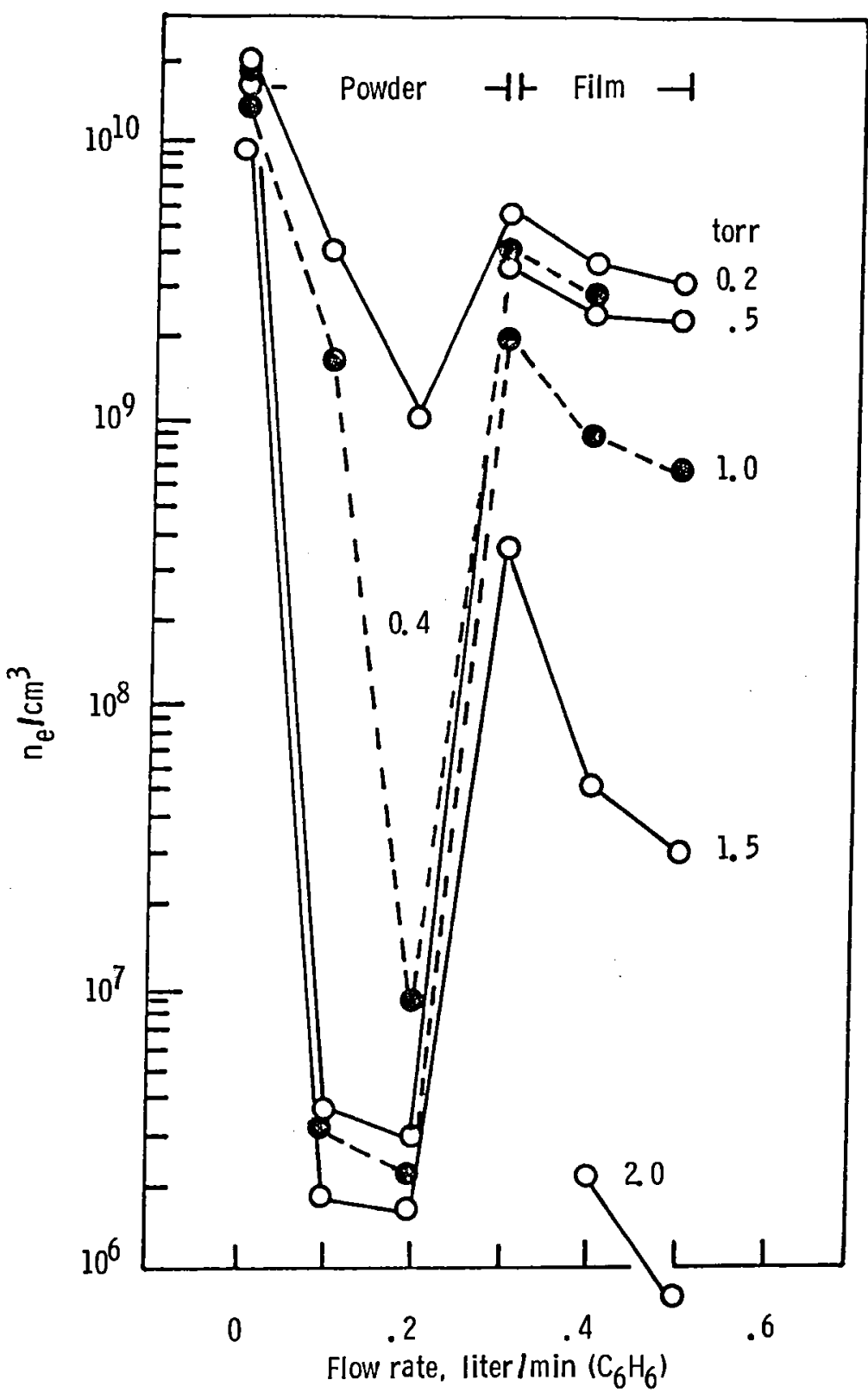

Figure 44. 


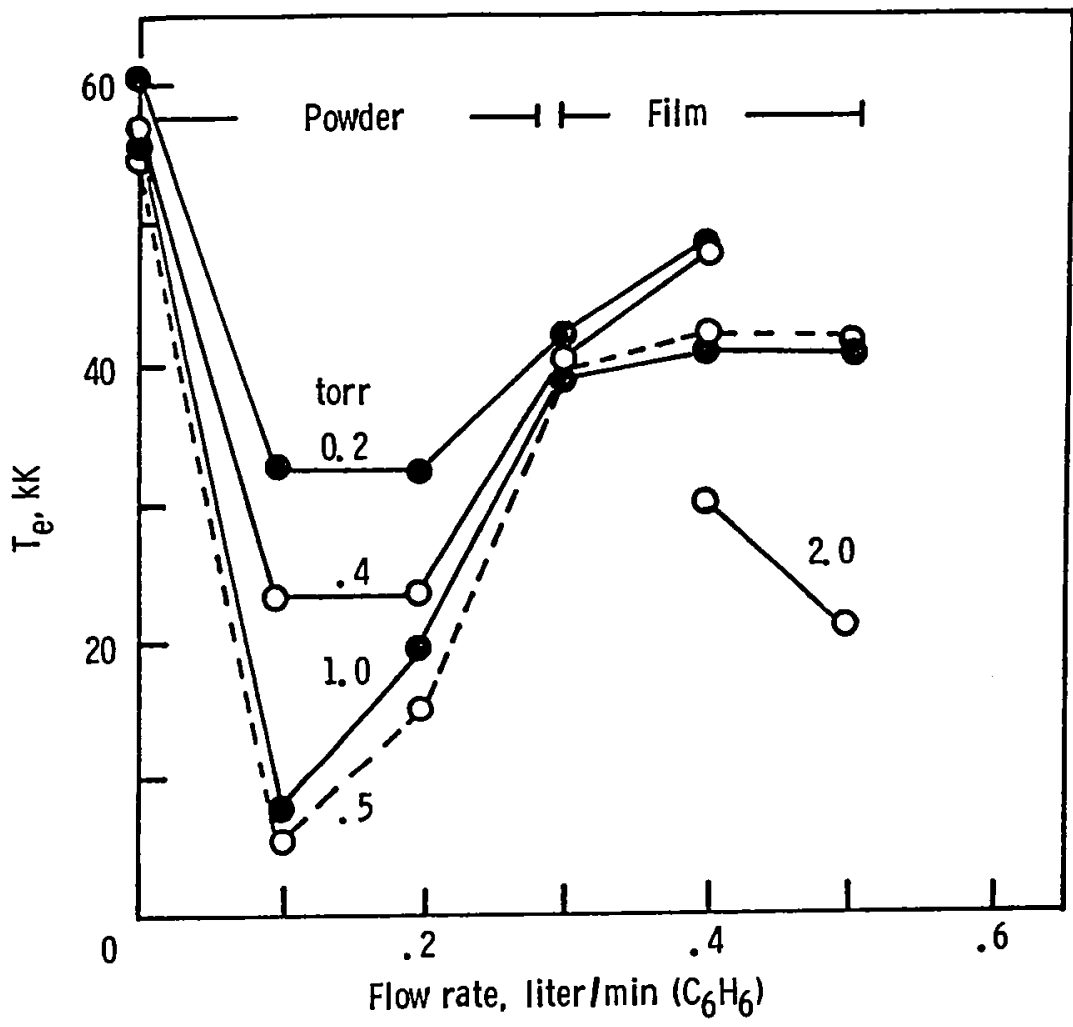

Figure 45. 


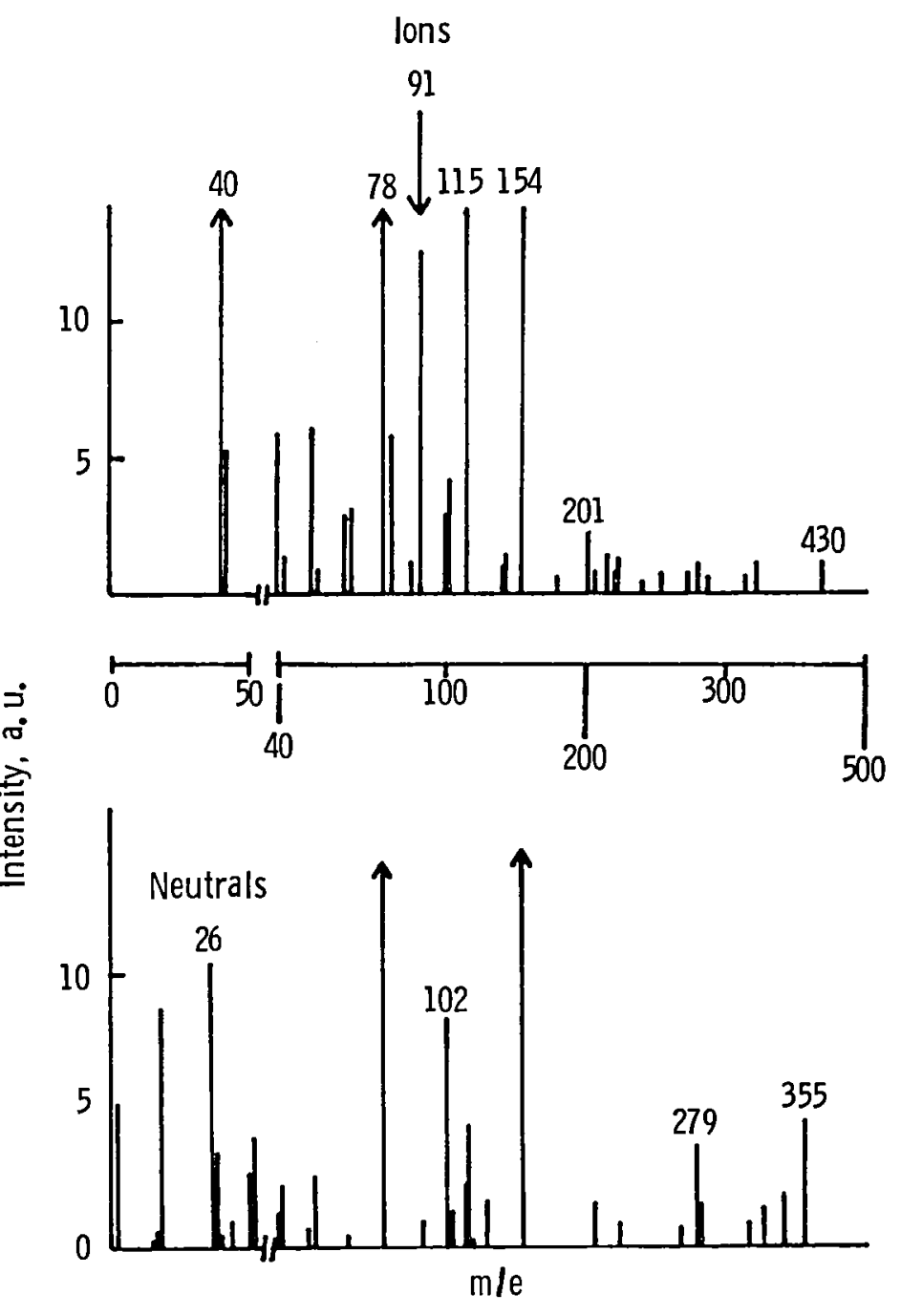

Figure 46. 


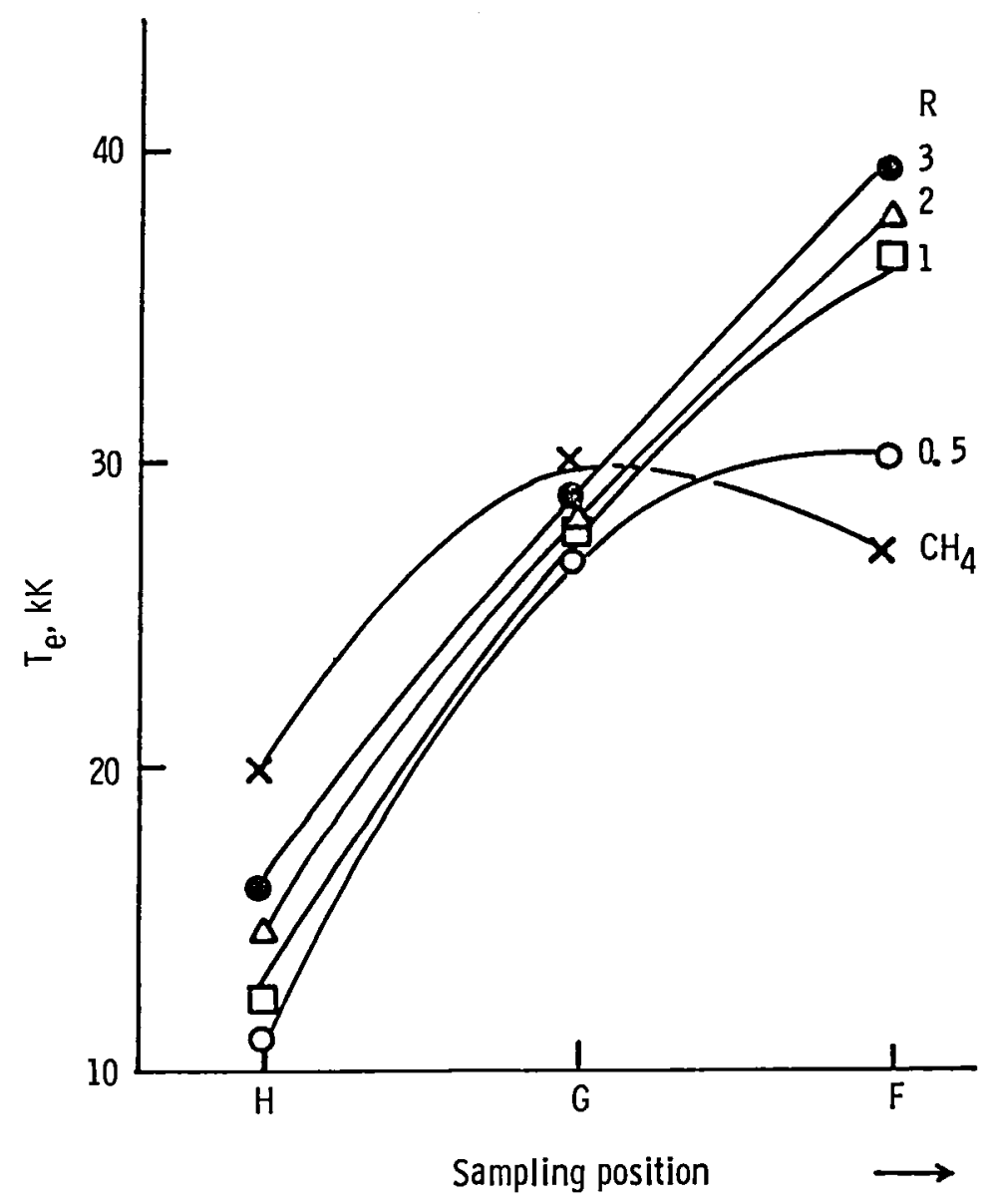

Figure 47. 


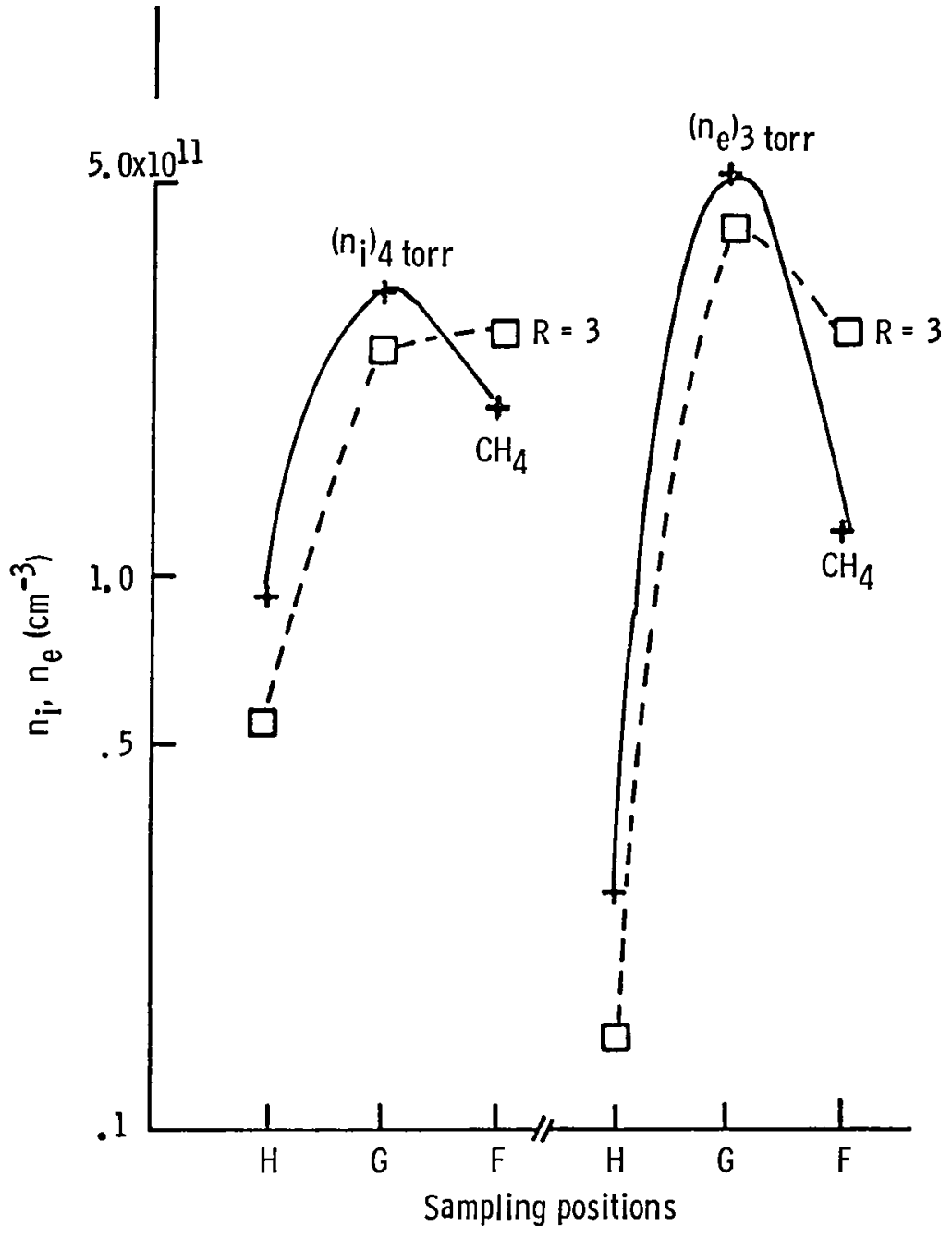

Figure 48. 


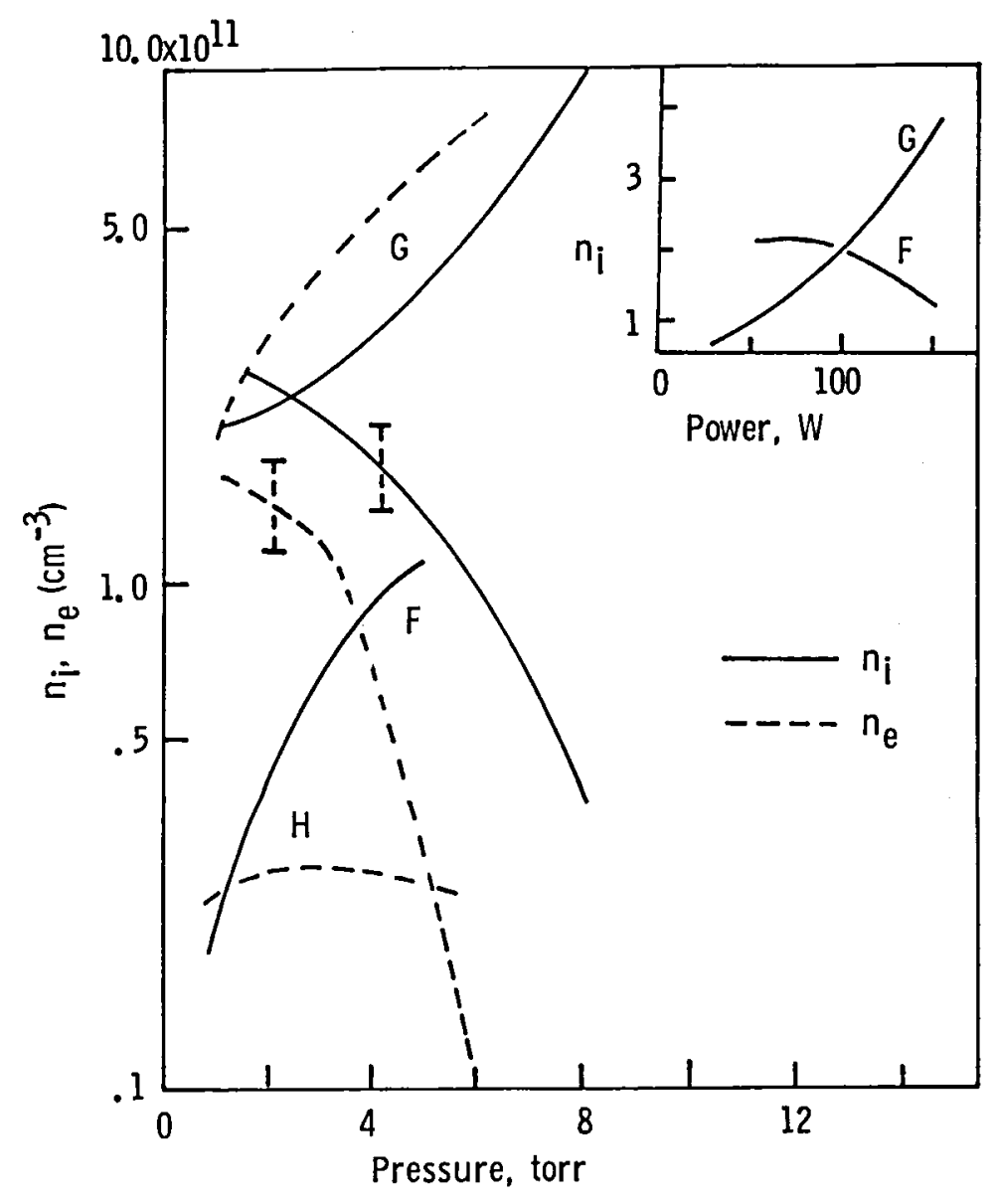

Figure 49. 


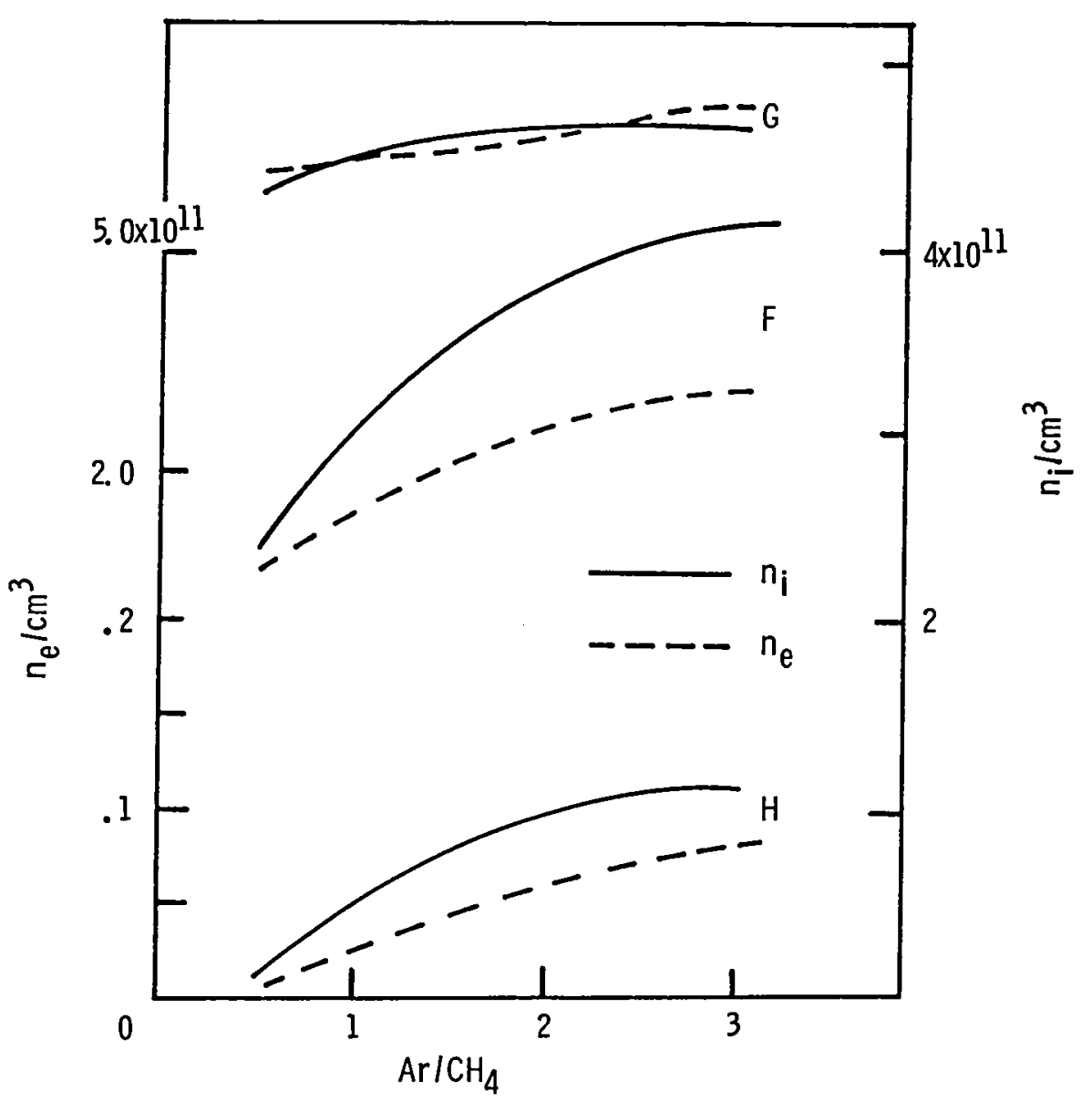

Figure 50. 


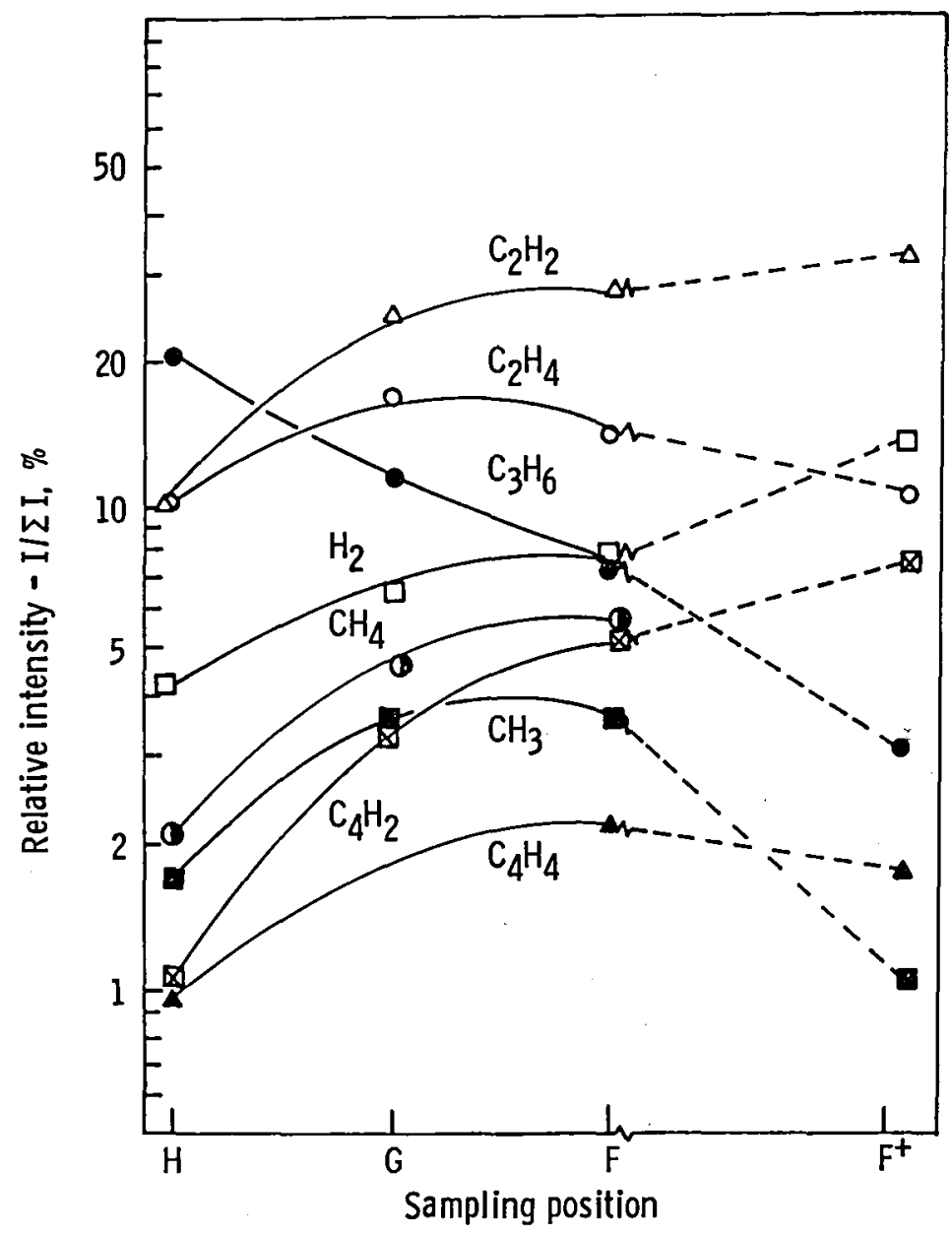

Figure 51.

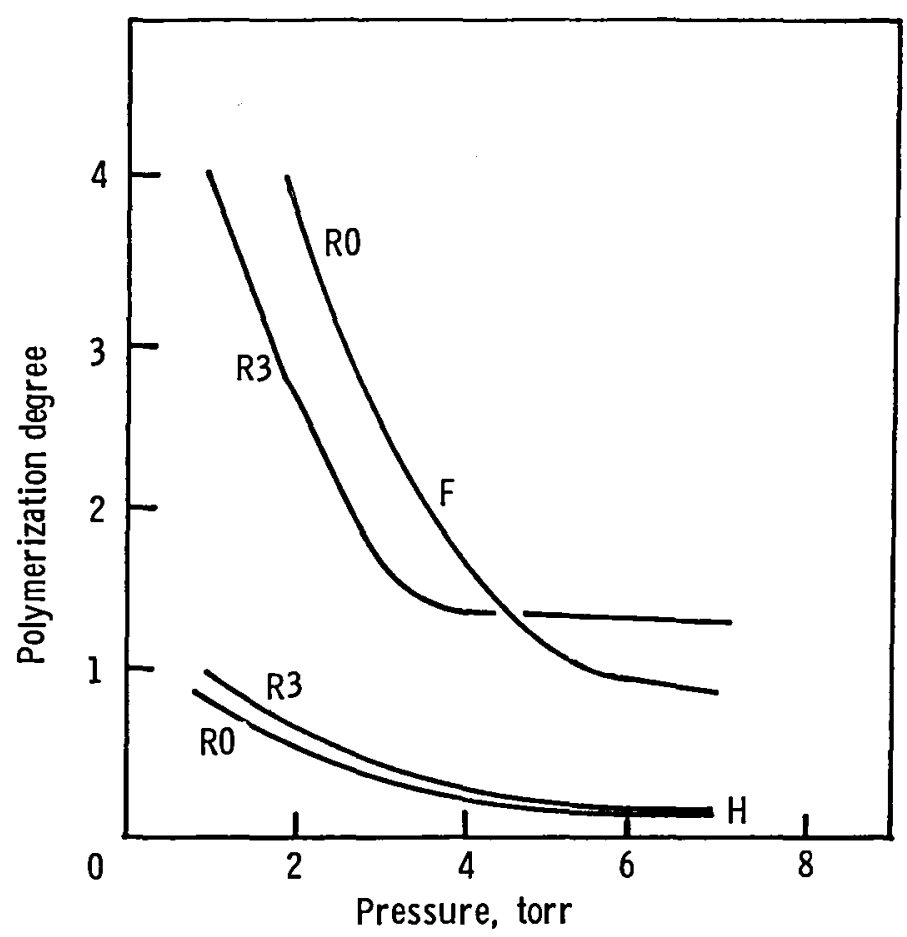

Figure 52. 


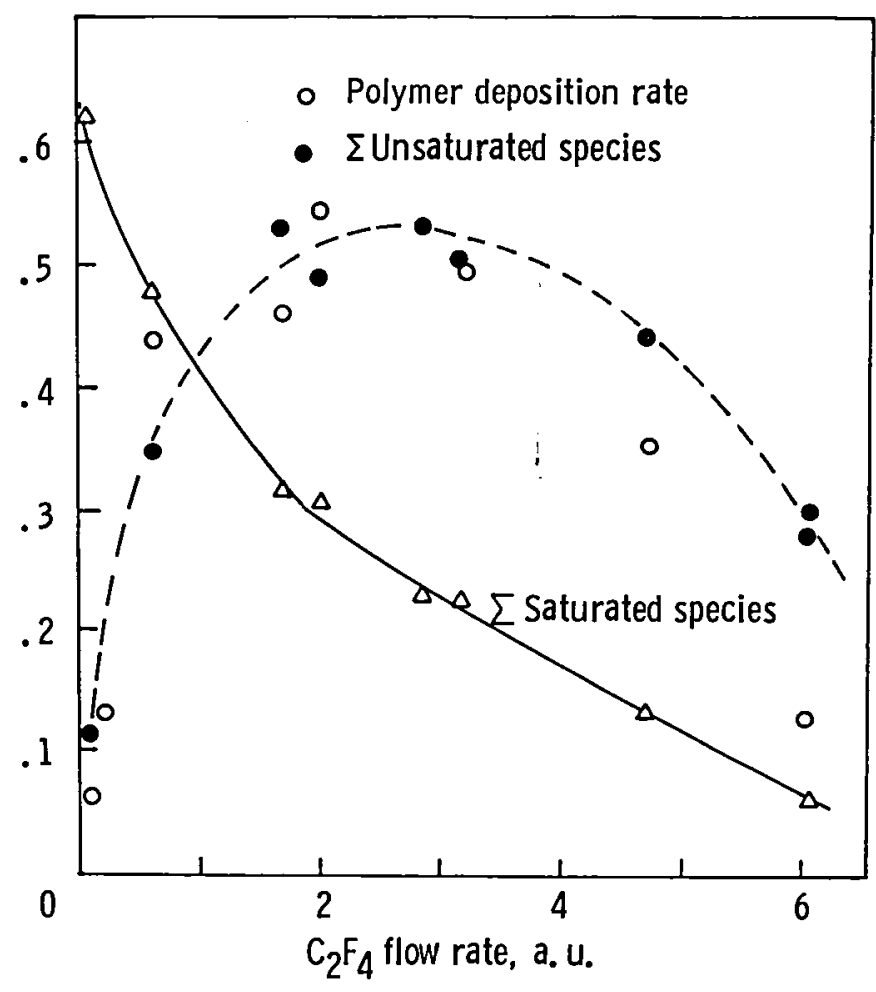

Figure 53.

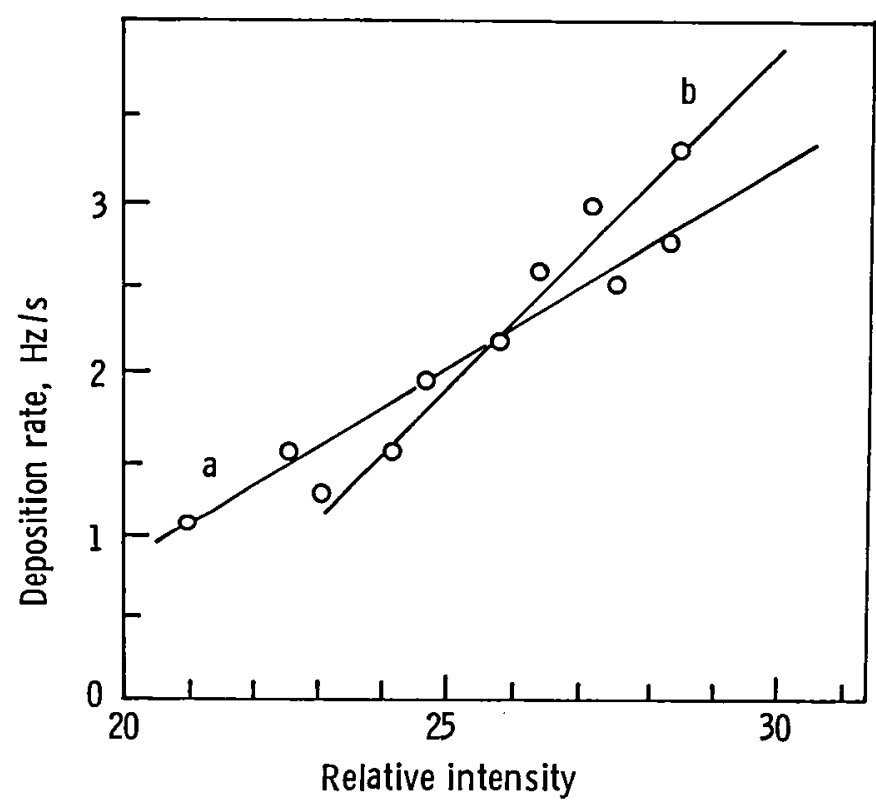

Figure 54. 


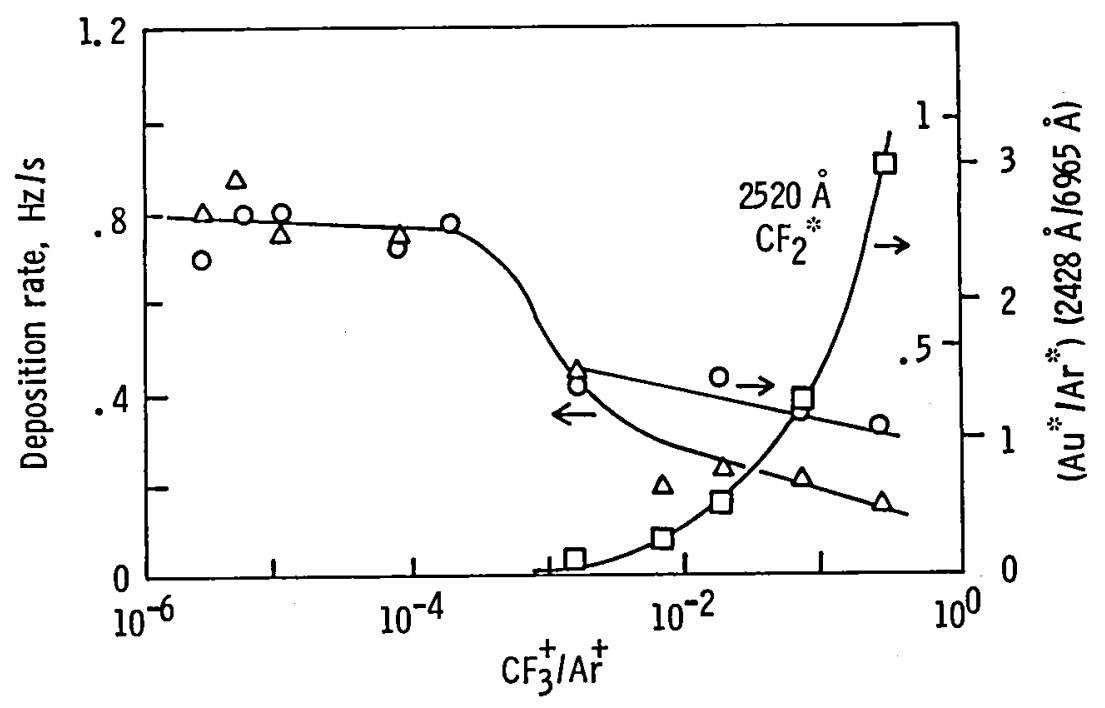

Figure 55.

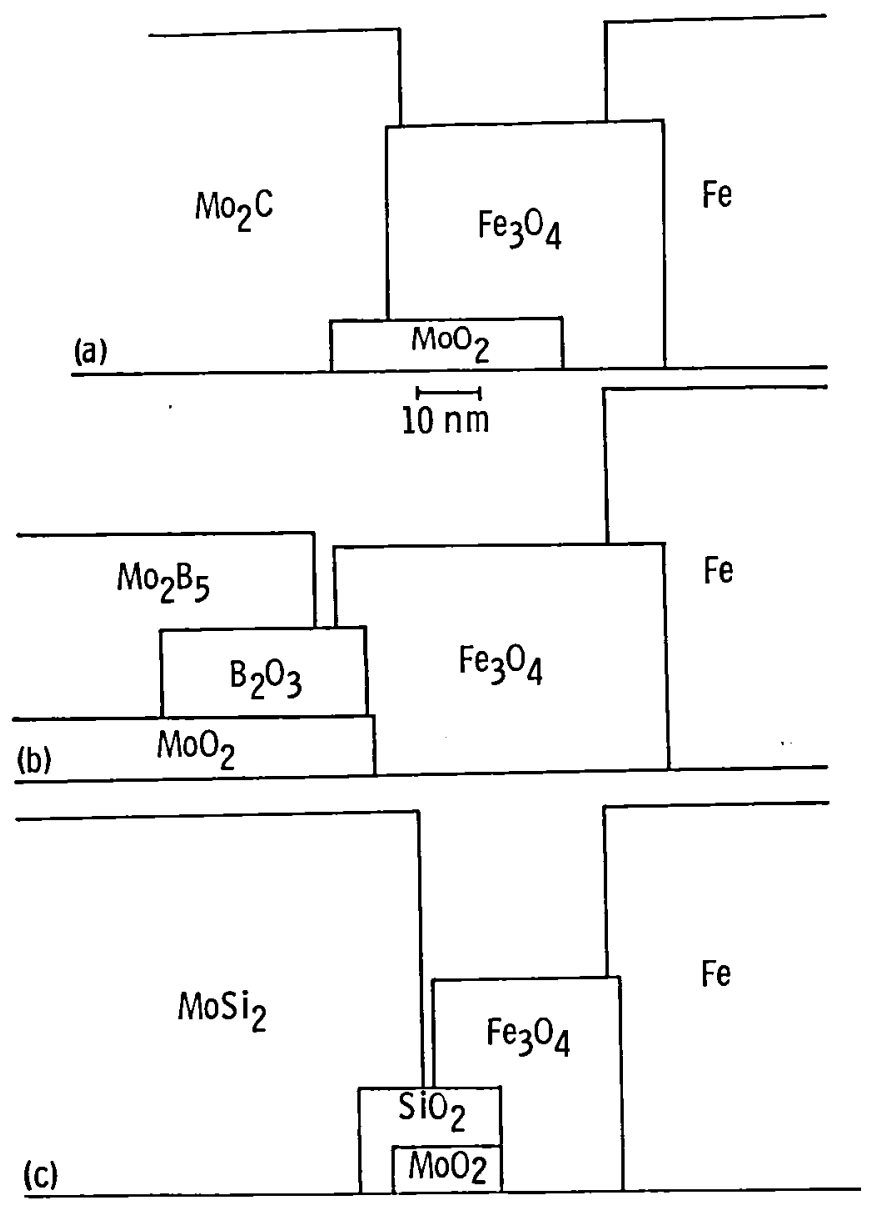

Figure 56. 


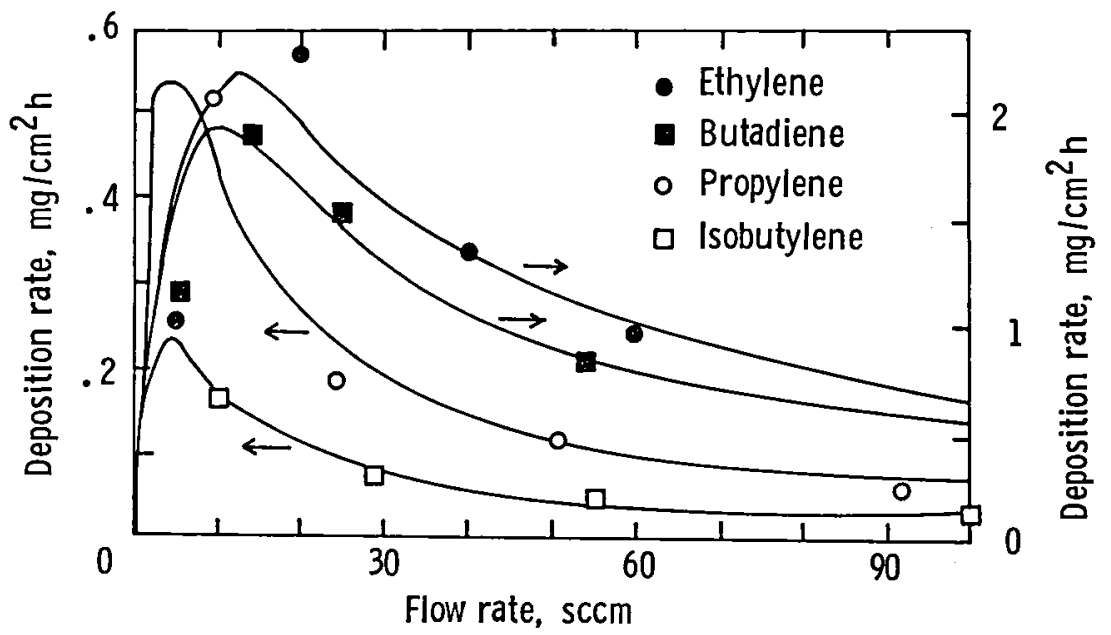

Figure 57.

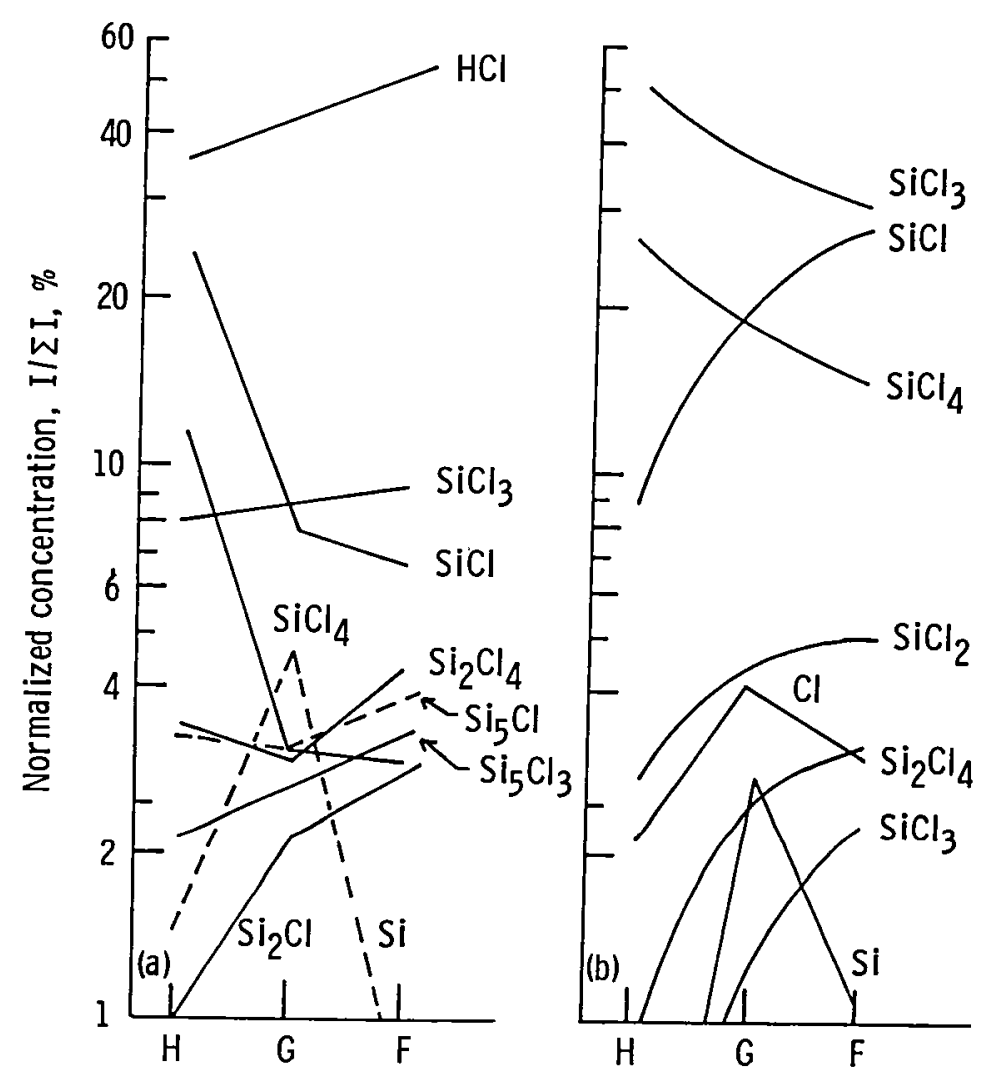

Figure 58. 


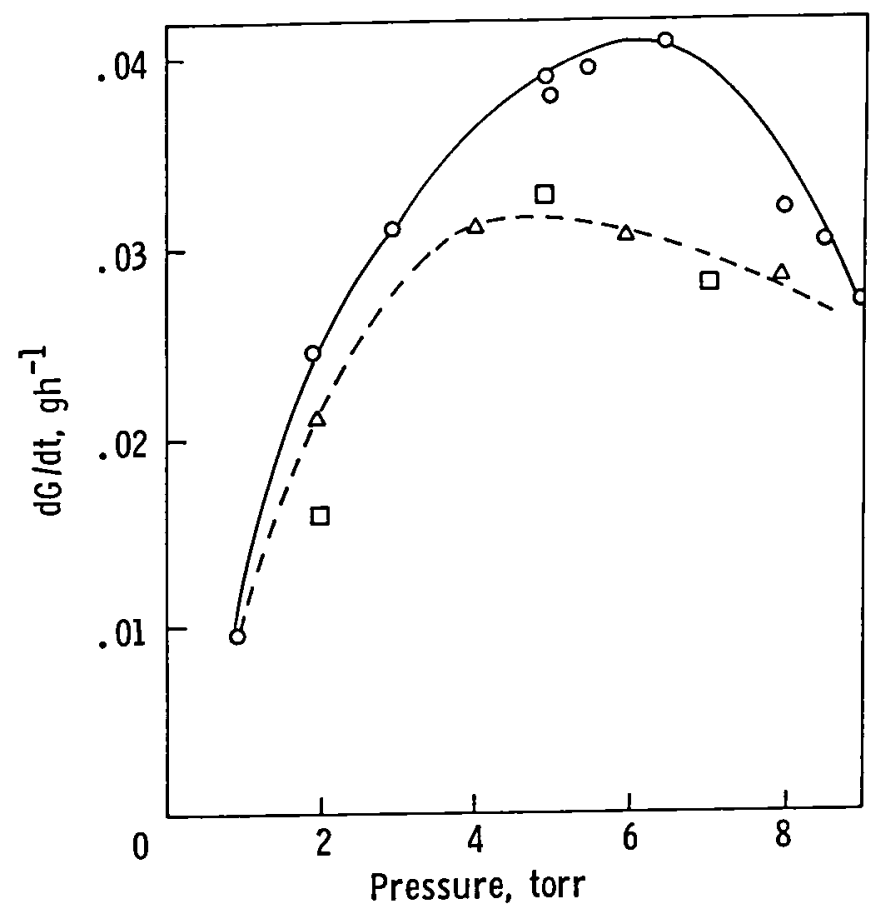

Figure 59.

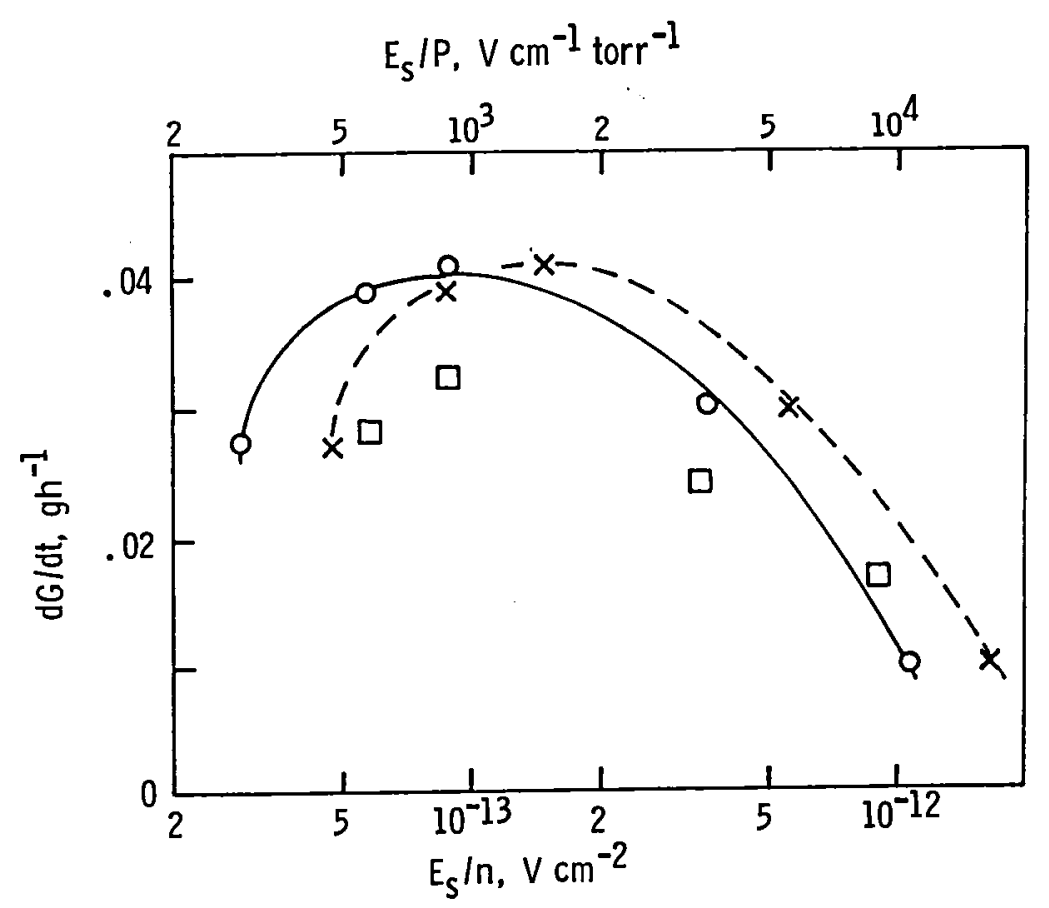

Figure 60. 


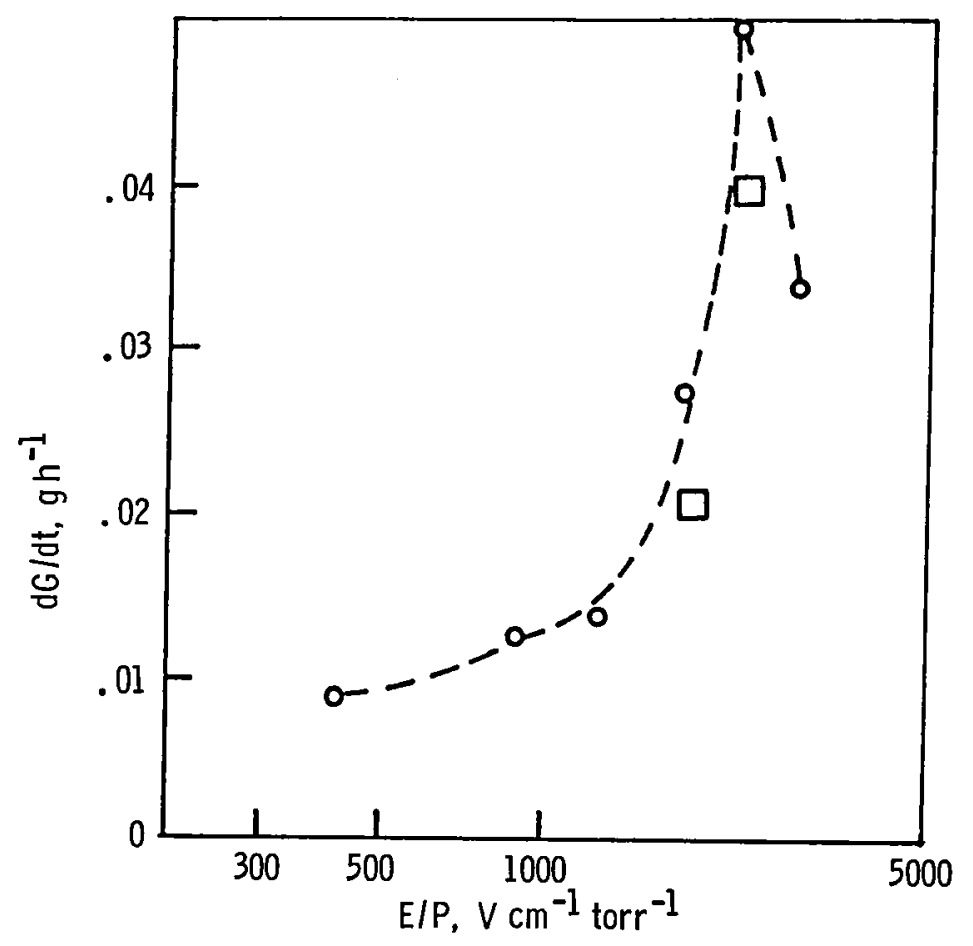

Figure 61. 


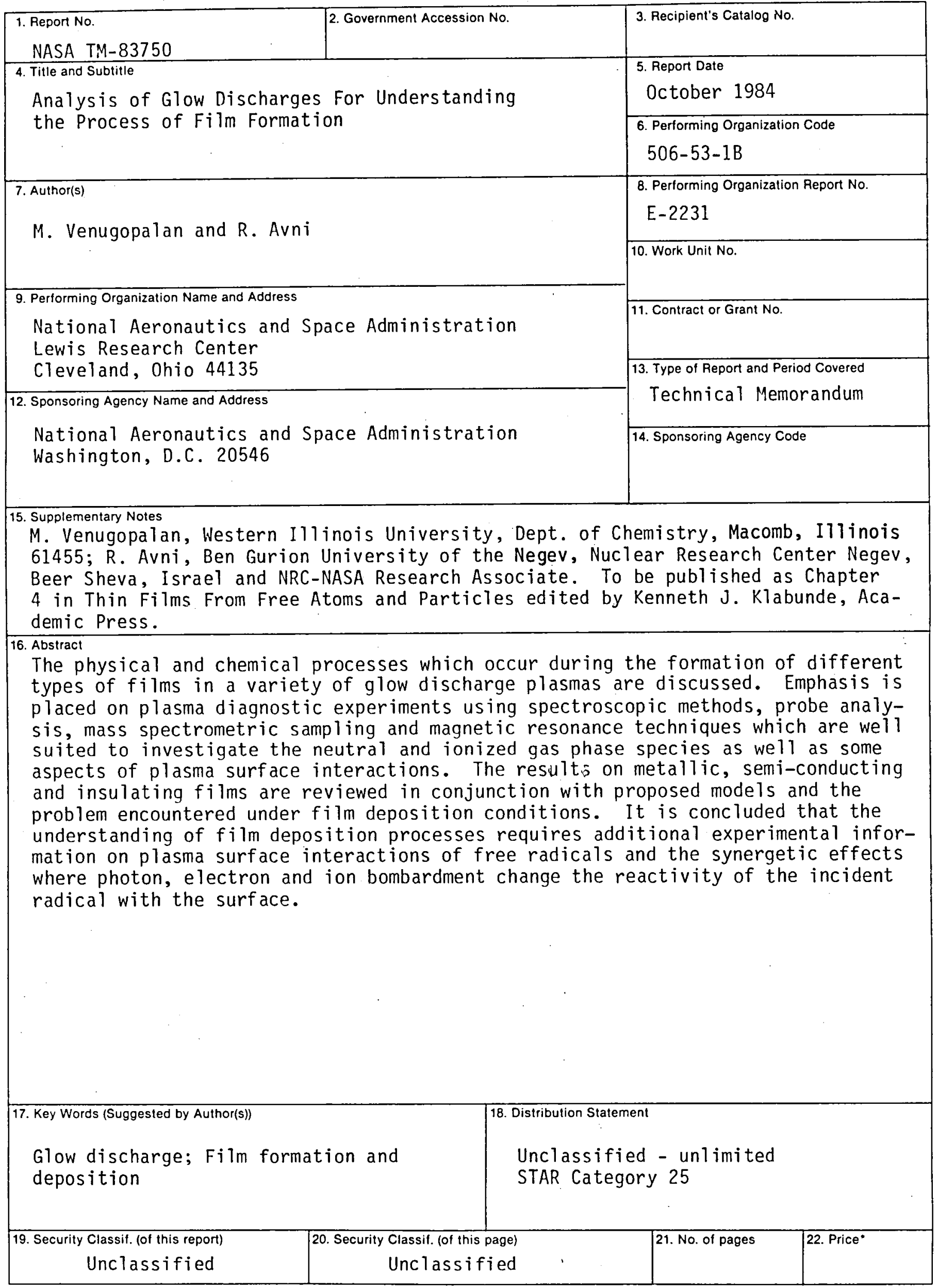

"For sale by the National Technical Information Service, Springfield, Virginia 22161 
-

$-$ 
National Aeronautics and Space Administration

Washington, D.C.

20546

Official Business

Penalty for Private Use, $\$ 300$
Si

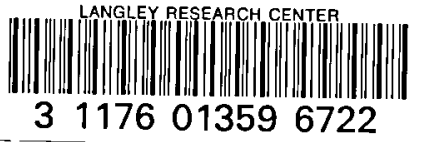

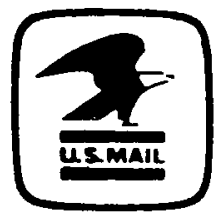

Postage and Fees Paid National Aeronautics and Space Administration NASA-451
DO NOT REMOVE SLIP FROM MATERIAL

Delete your name from this slip when returning material to the library.

\begin{tabular}{|c|c|c|}
\hline NAME & DATE & MS \\
\hline Dongchuan wu & $5 / 3 / 92$ & 493 \\
\hline & & \\
\hline & & \\
\hline & & \\
\hline & & \\
\hline & & \\
\hline & & \\
\hline & & \\
\hline & & \\
\hline NASA Langley (Rev. Doc. 1991) & & RIAD N-75
\end{tabular}

If Undeliverable (Section $15 x$ Postal Manual) (k) Not Return 